\title{
Asymmetric Palladium-Catalyzed C-H Functionalization Cascade for Synthesis of Chiral 3,4-Dihydroisoquinolones
}

Manman Sun, Haijian Wu, Xiangyu Xia, Weida Chen, Zhiming Wang, and Jianguo

$$
\text { Yang* }
$$

Advanced Research Institute and Department of Chemistry, Taizhou University, 1139 Shifu Avenue, Taizhou 318000, P. R. China.E-mail:yjg@tzc.edu.cn

\section{Supporting Information}

Table of Contents

Table S1. Optimization of Pd-catalysts and solvents $\ldots \ldots \ldots \ldots \ldots . . . . . . . . . . . . . . . . .52$

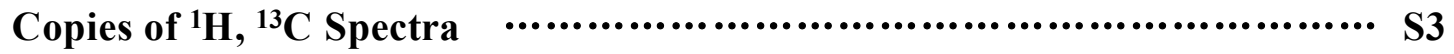

HPLC Charts of related compounds $\quad$...................................... S53

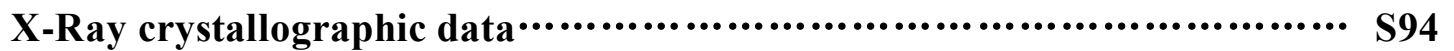


Table S1. Optimization of Pd-catalysts and solvents. ${ }^{a}$
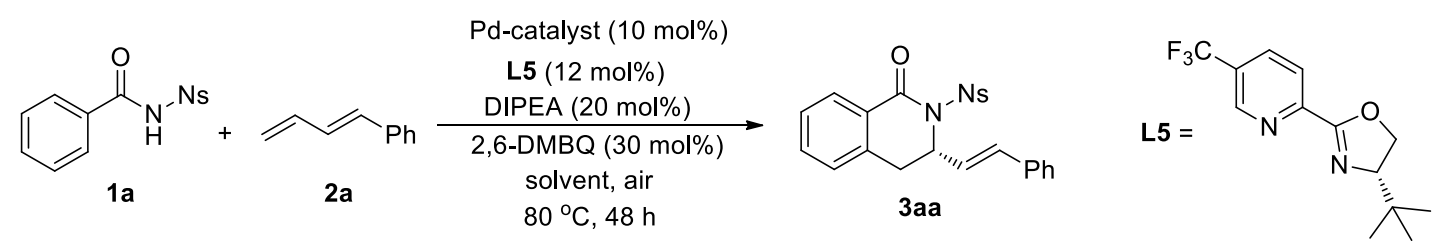

\begin{tabular}{lllll}
\hline entry & Pd-catalyst & solvent & yield $(\%)^{b}$ & ee $(\%)^{c}$ \\
\hline 1 & $\mathrm{Pd}(\mathrm{MeCN})_{2}(\mathrm{OTs})_{2}$ & $\mathrm{PhCF}_{3}$ & 56 & 85 \\
2 & $\mathrm{Pd}(\mathrm{OAc})_{2}$ & $\mathrm{PhCF}_{3}$ & 76 & 85 \\
3 & $\mathrm{PdCl}_{2}$ & $\mathrm{PhCF}_{3}$ & trace & - \\
4 & $\mathrm{Pd}(\mathrm{TFA})_{2}$ & toluene & 72 & 78 \\
5 & $\mathrm{Pd}(\mathrm{TFA})_{2}$ & chlorobenzene & 77 & 80 \\
6 & $\mathrm{Pd}(\mathrm{TFA})_{2}$ & $1,4-$ dioxane & trace & - \\
7 & $\mathrm{Pd}(\mathrm{TFA})_{2}$ & $\mathrm{MeCN}$ & trace & -
\end{tabular}

${ }^{a}$ Reaction conditions: 1a $(0.1 \mathrm{mmol}), 2 \mathrm{a}(0.2 \mathrm{mmol})$, Pd-catalyst $(0.01 \mathrm{mmol}), \mathbf{L 5}$ (0.012 mmol), DIPEA (0.02 mmol) and 2,6-DMBQ $(0.03 \mathrm{mmol})$ in solvent $(0.2 \mathrm{~mL})$ for $48 \mathrm{~h}$ under air. ${ }^{b}$ Isolated yield. ${ }^{c}$ Determined by HPLC. 


\section{Copies of ${ }^{1} \mathbf{H},{ }^{13} \mathrm{C}$ Spectra}

$1 c$

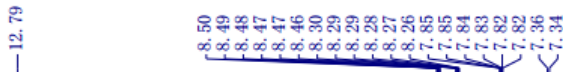

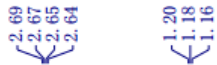
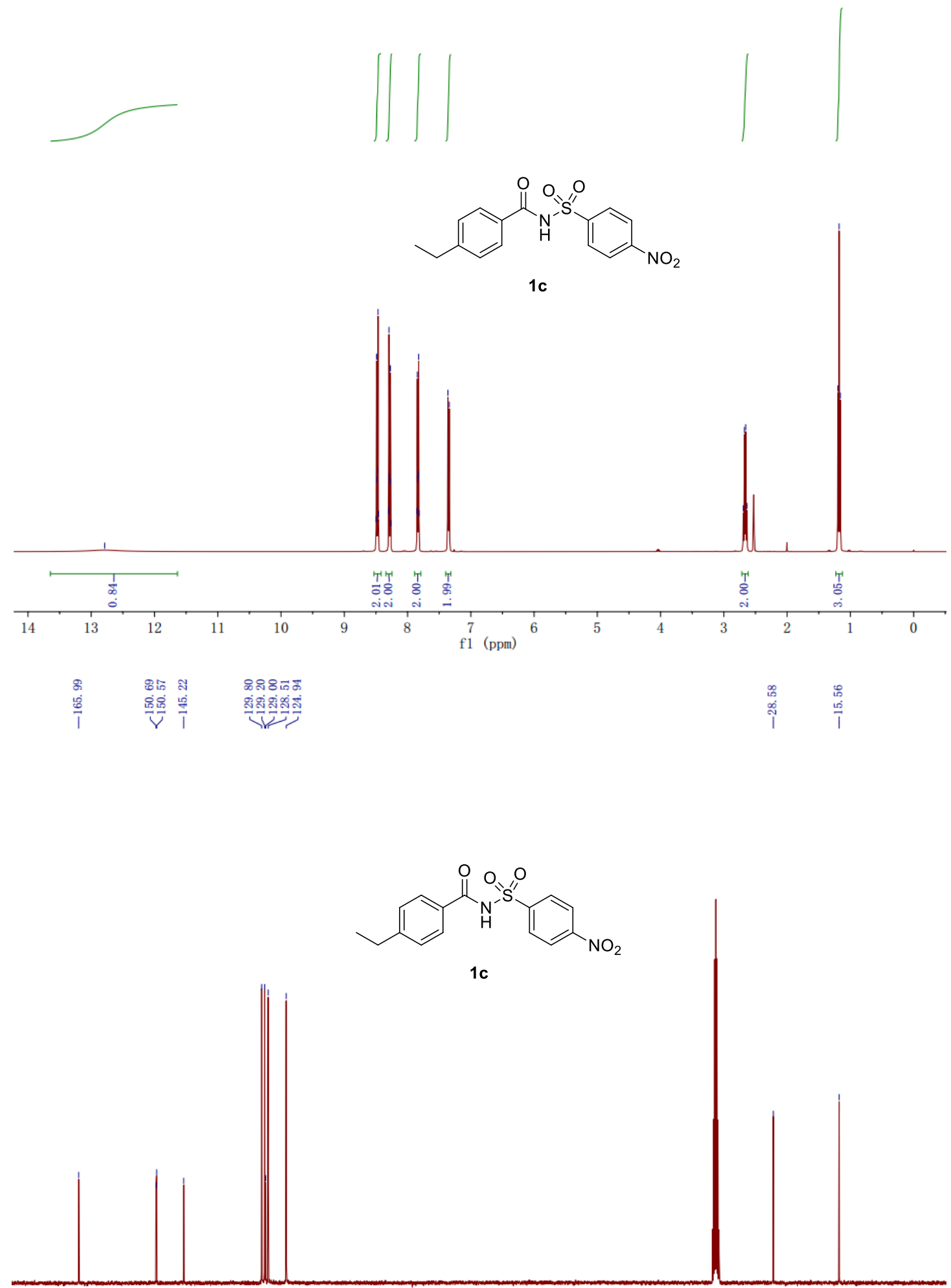

\begin{tabular}{llllllllllllllllllllll}
\hline 0 & 170 & 160 & 150 & 140 & 130 & 120 & 110 & 100 & 90 & 80 & 70 & 60 & 50 & 40 & 30 & 20 & 10 & 0
\end{tabular} 


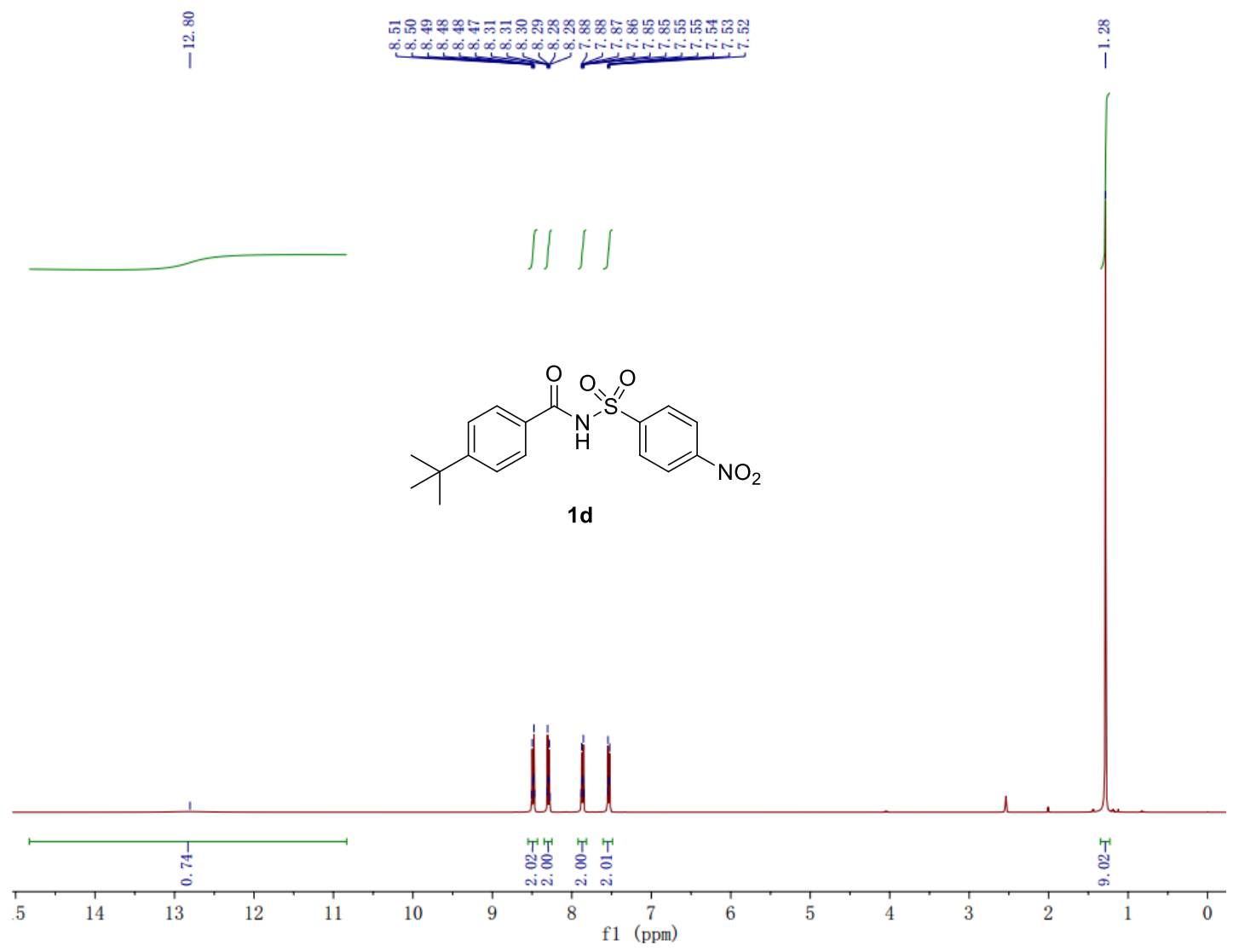

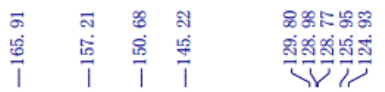

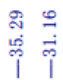

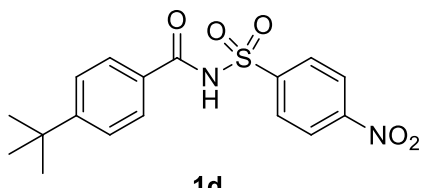

1d

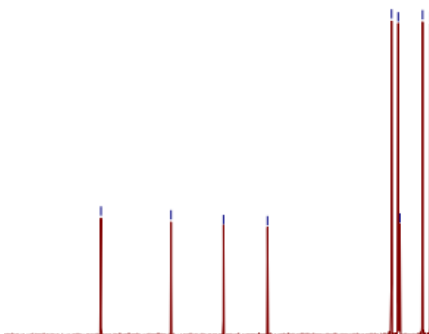

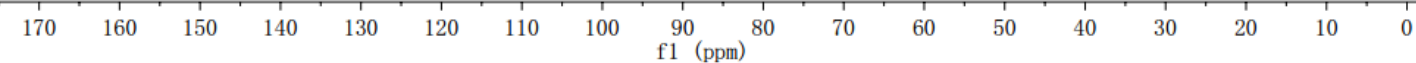


$1 \mathrm{~h}$
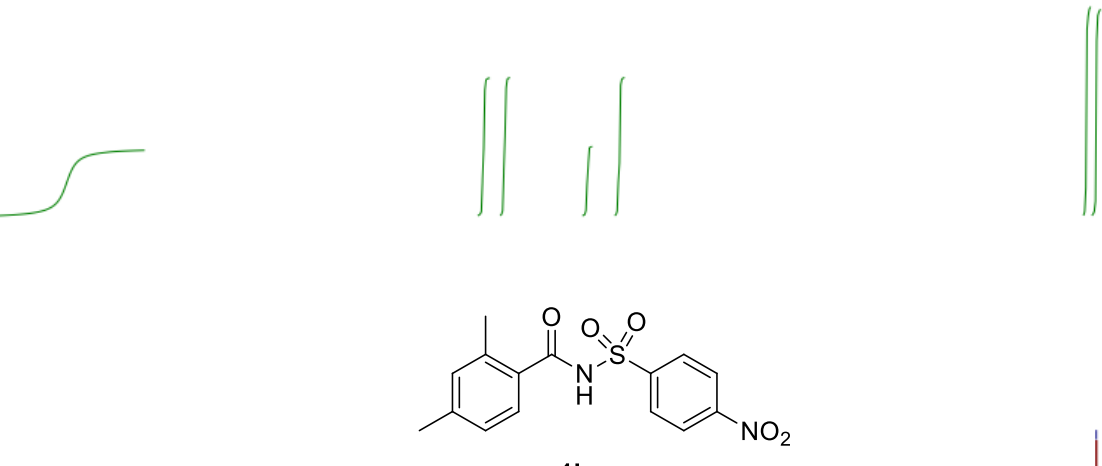

1h

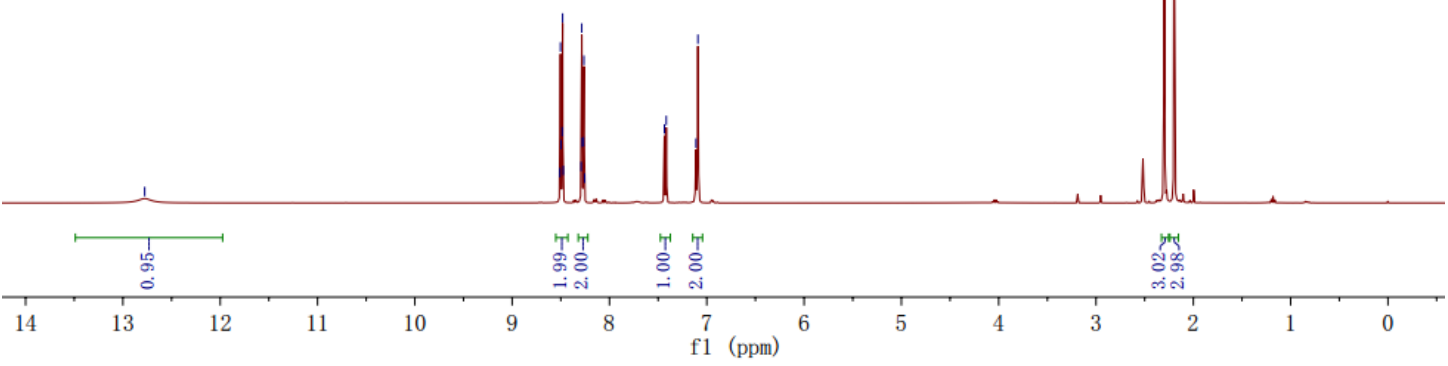

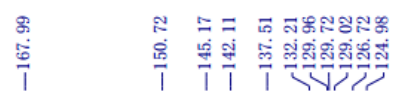

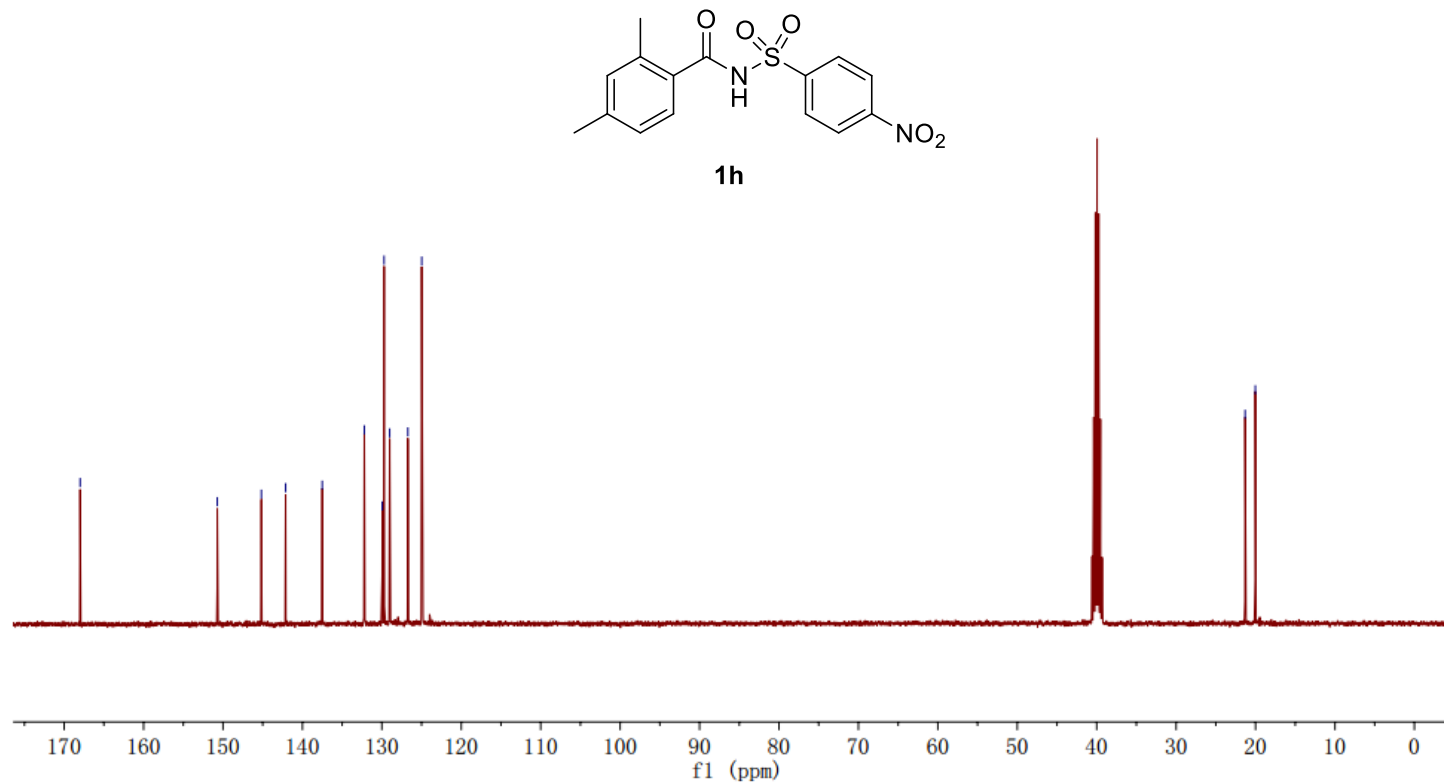


11

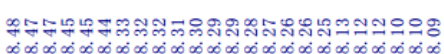

8
9
1
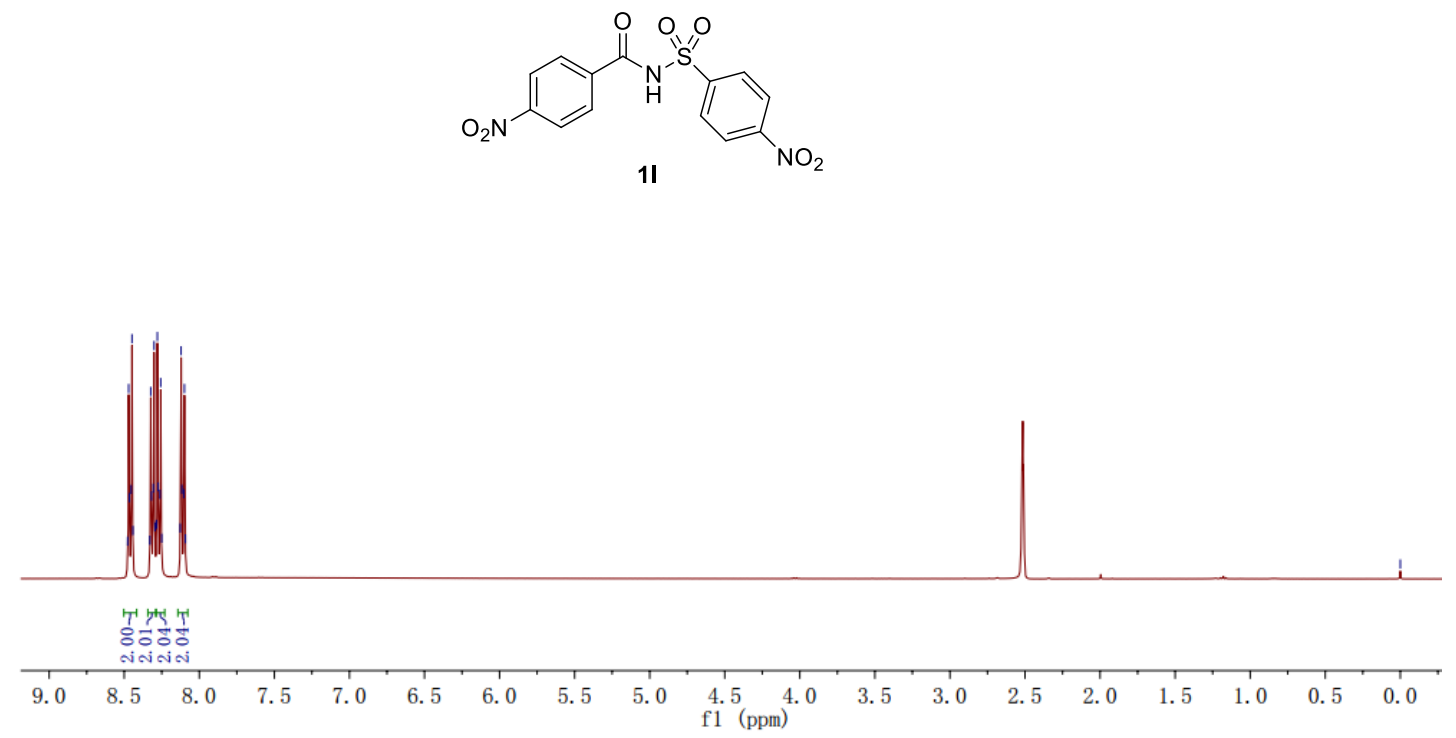

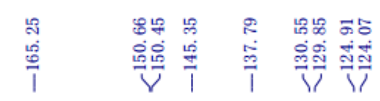
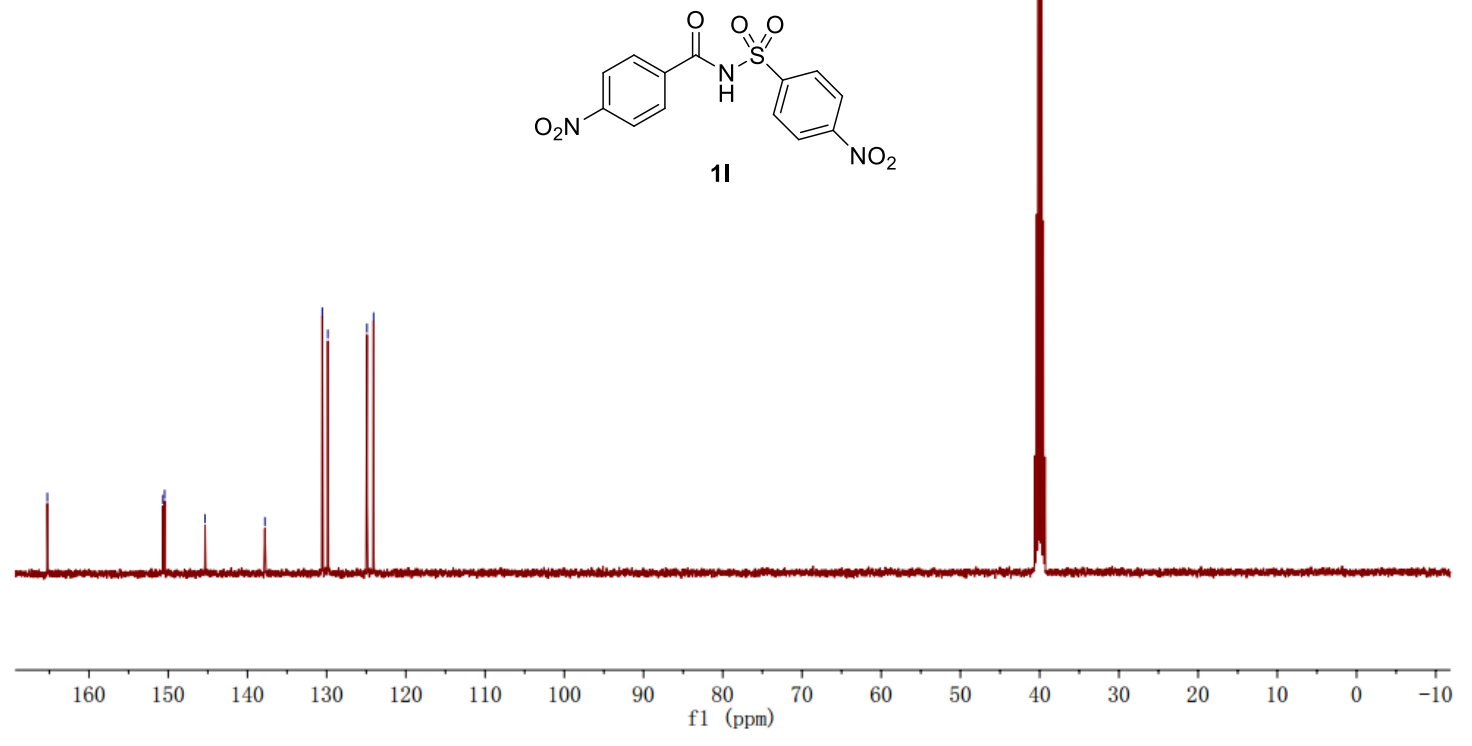

S6 
$1 \mathrm{~m}$

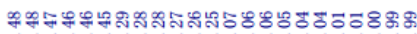

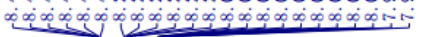

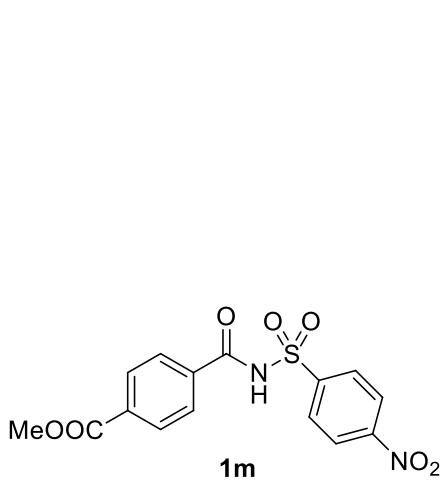

11
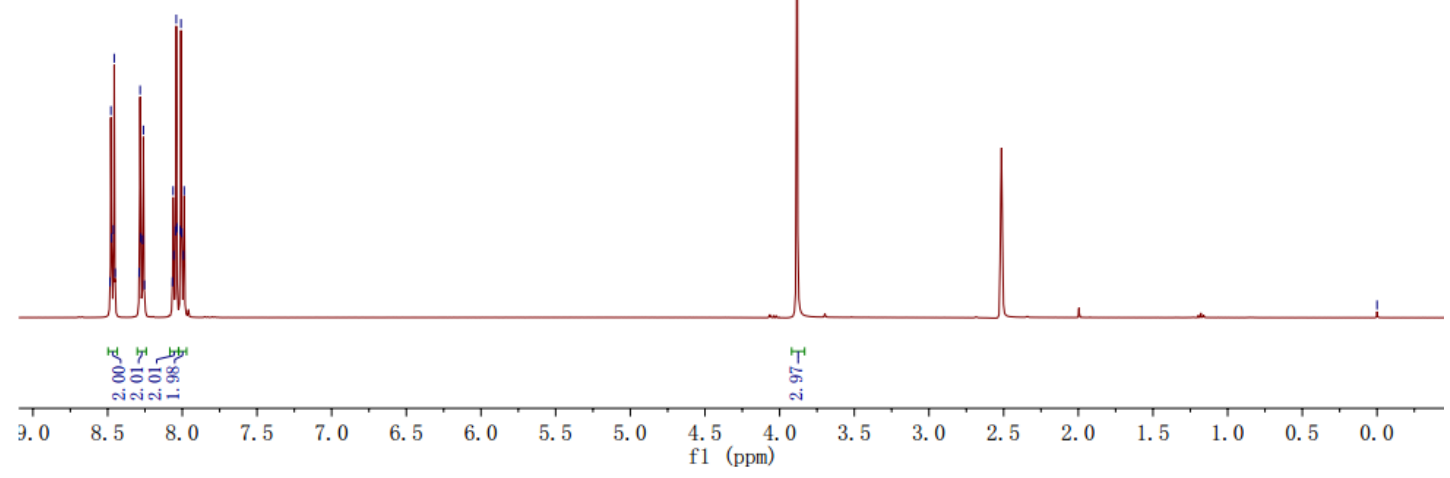

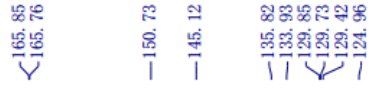
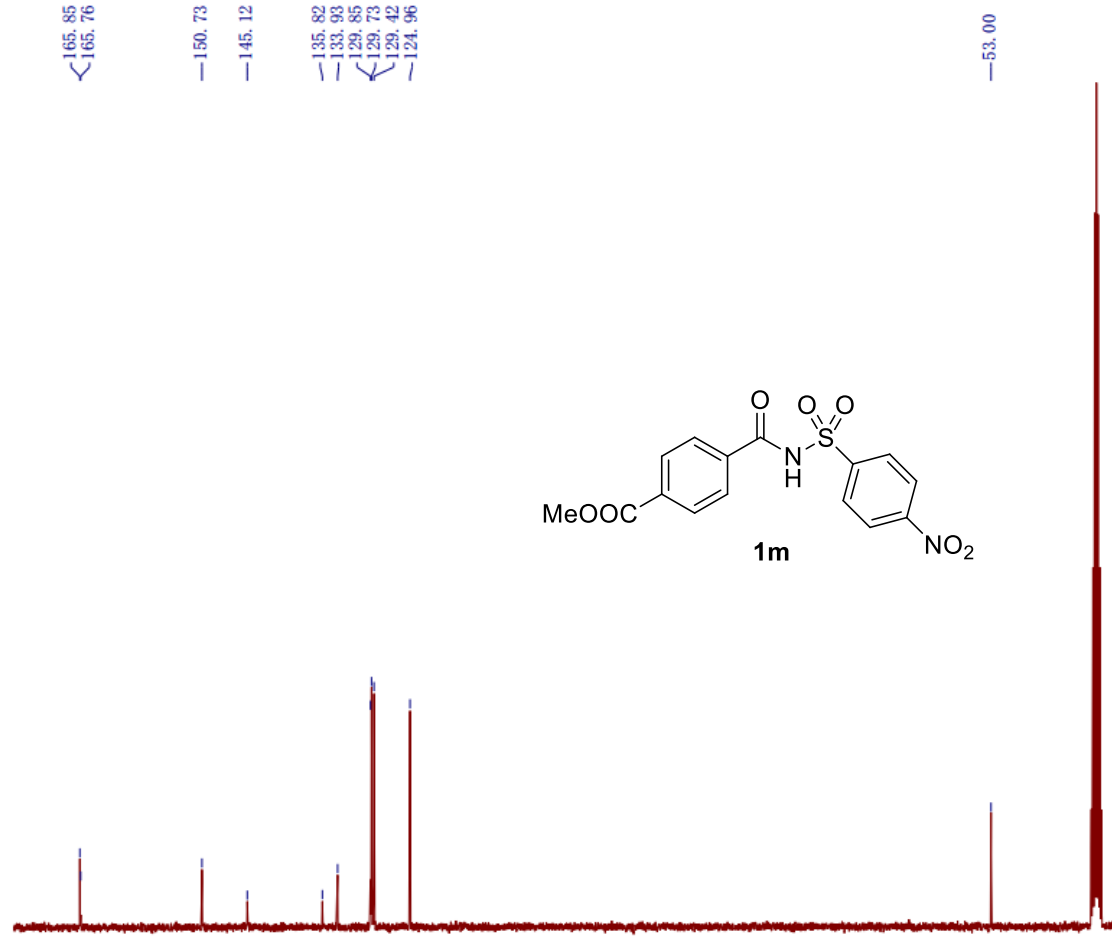

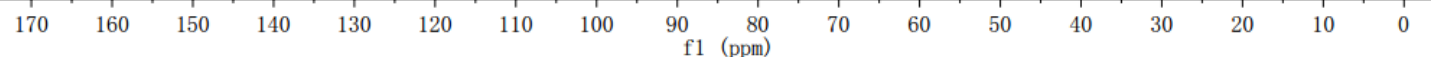




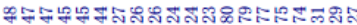
-

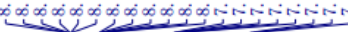
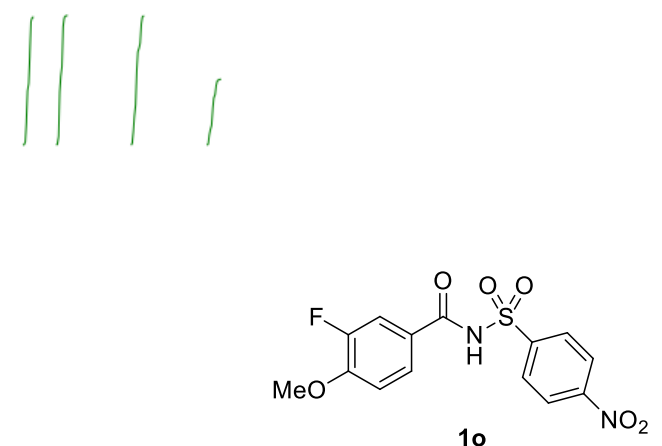

10
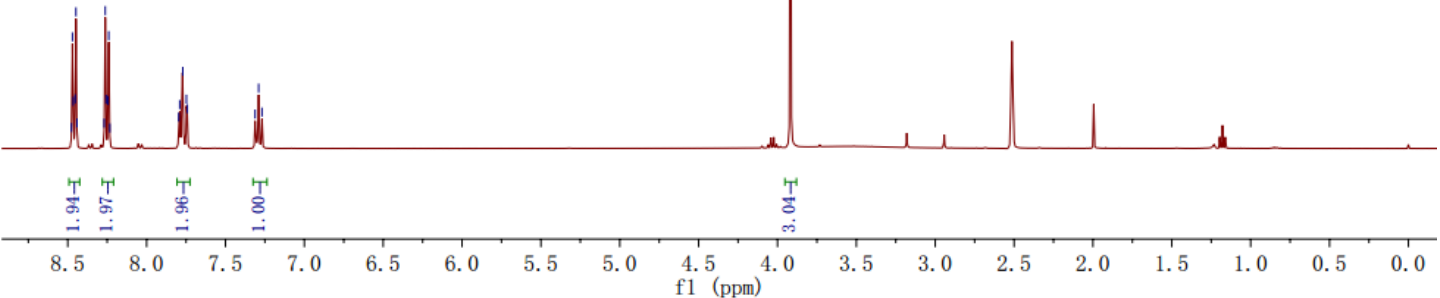

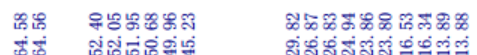

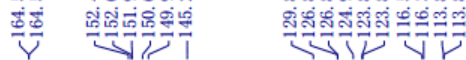
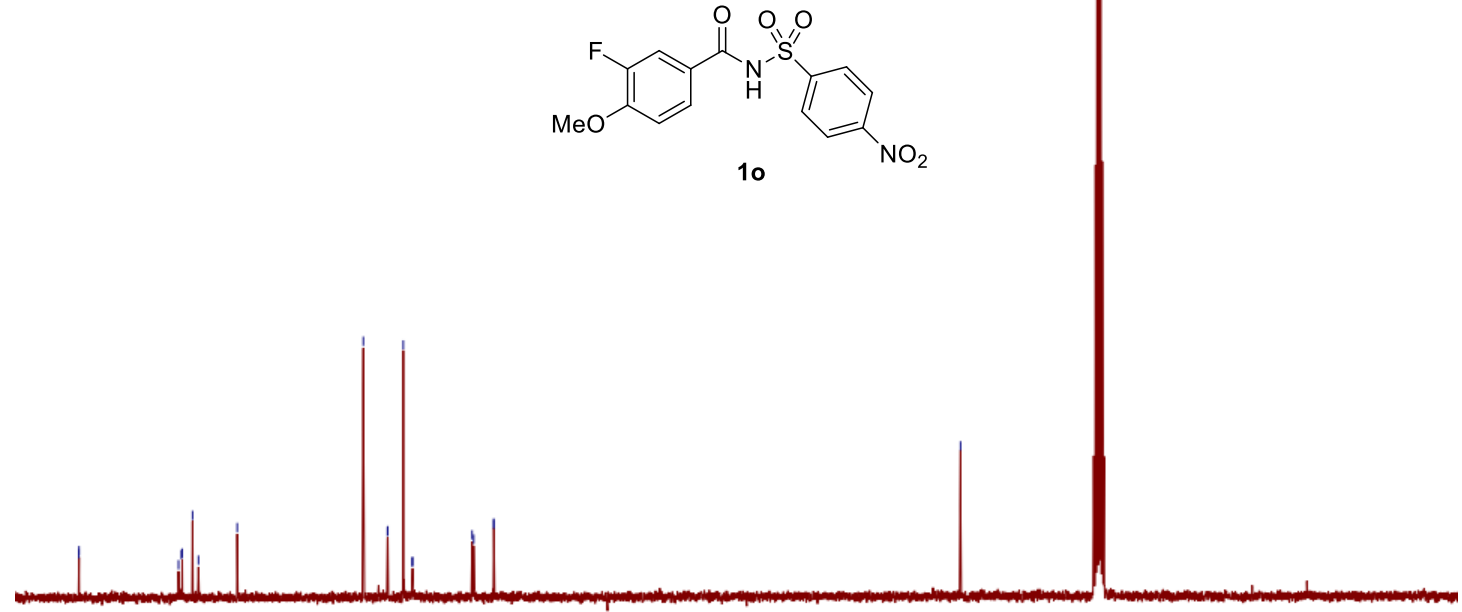

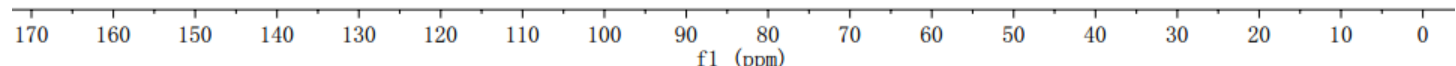


1 u

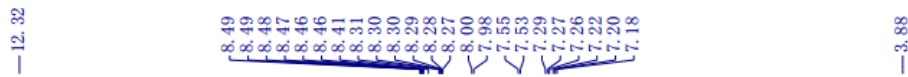

$\int$
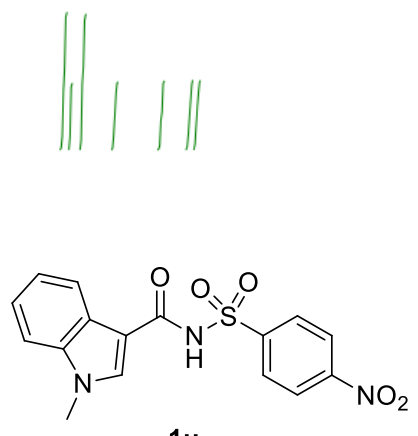

$1 u$

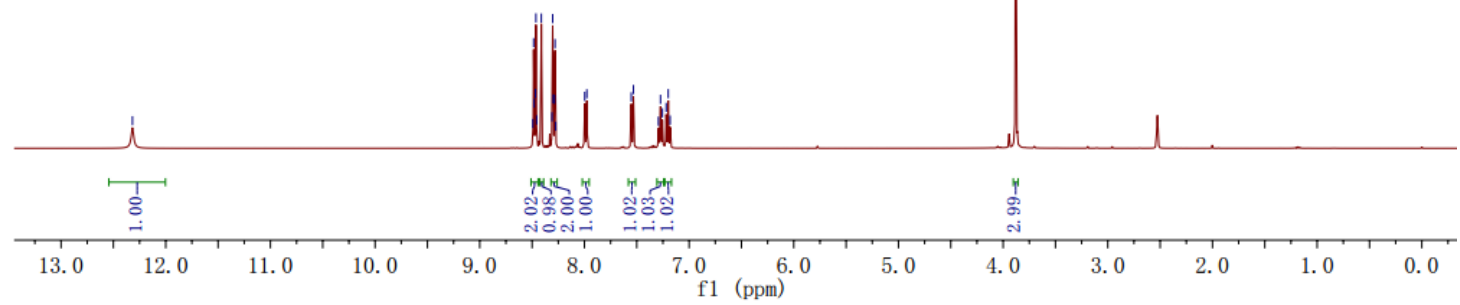

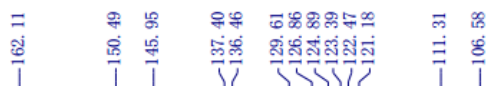

离

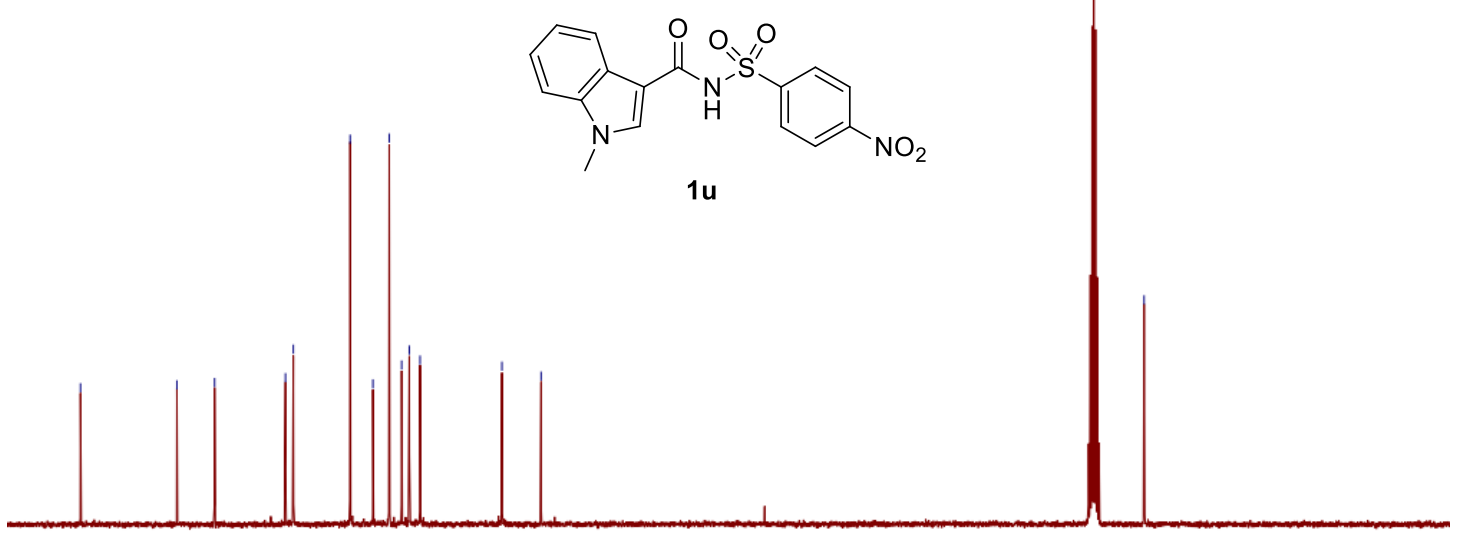

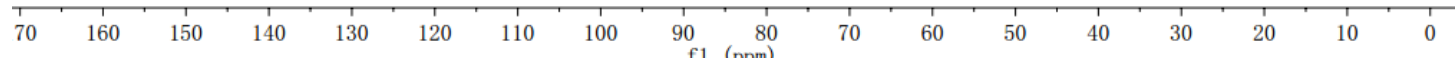




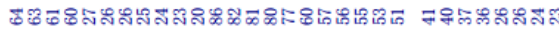

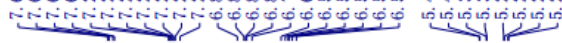
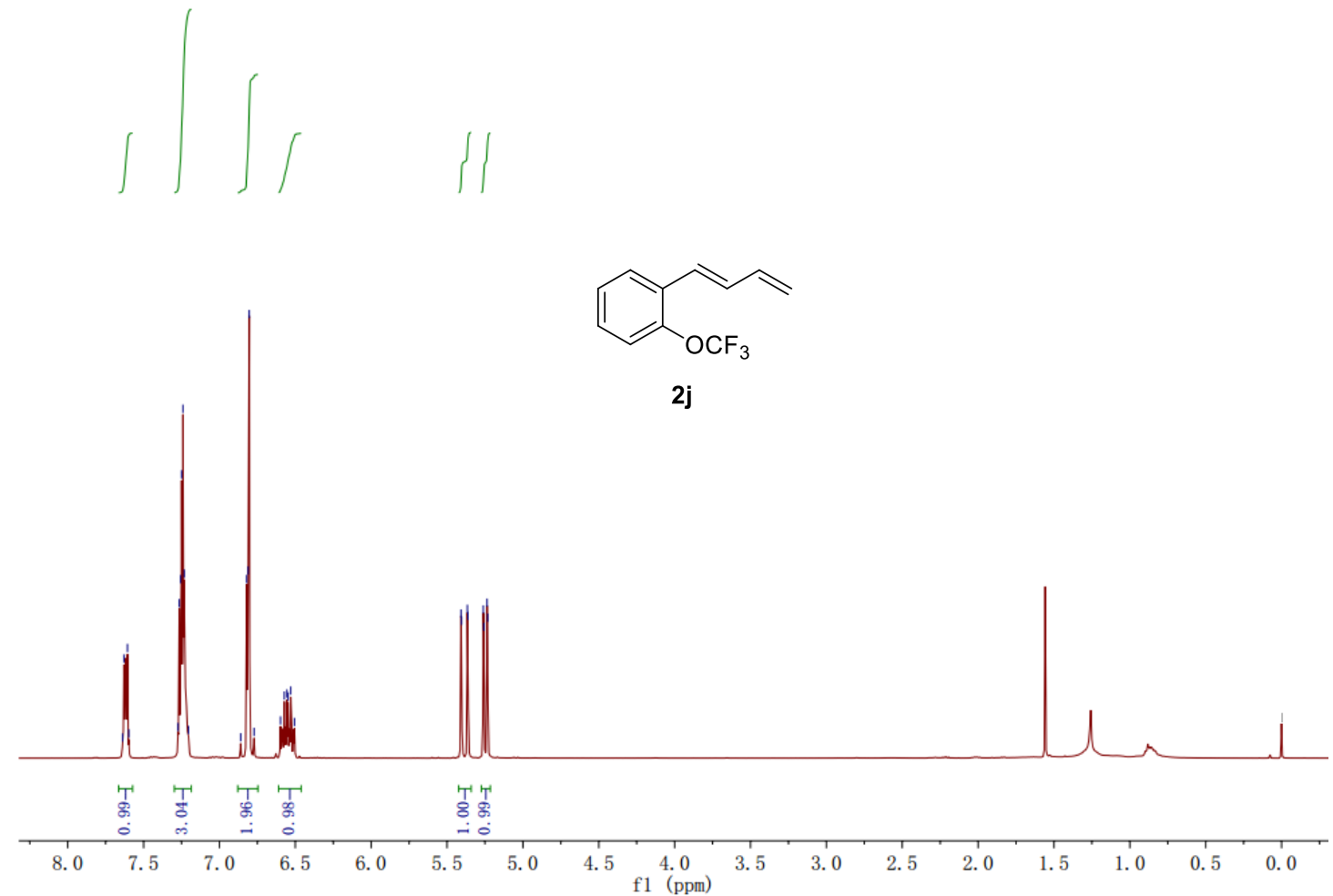

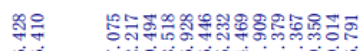

它

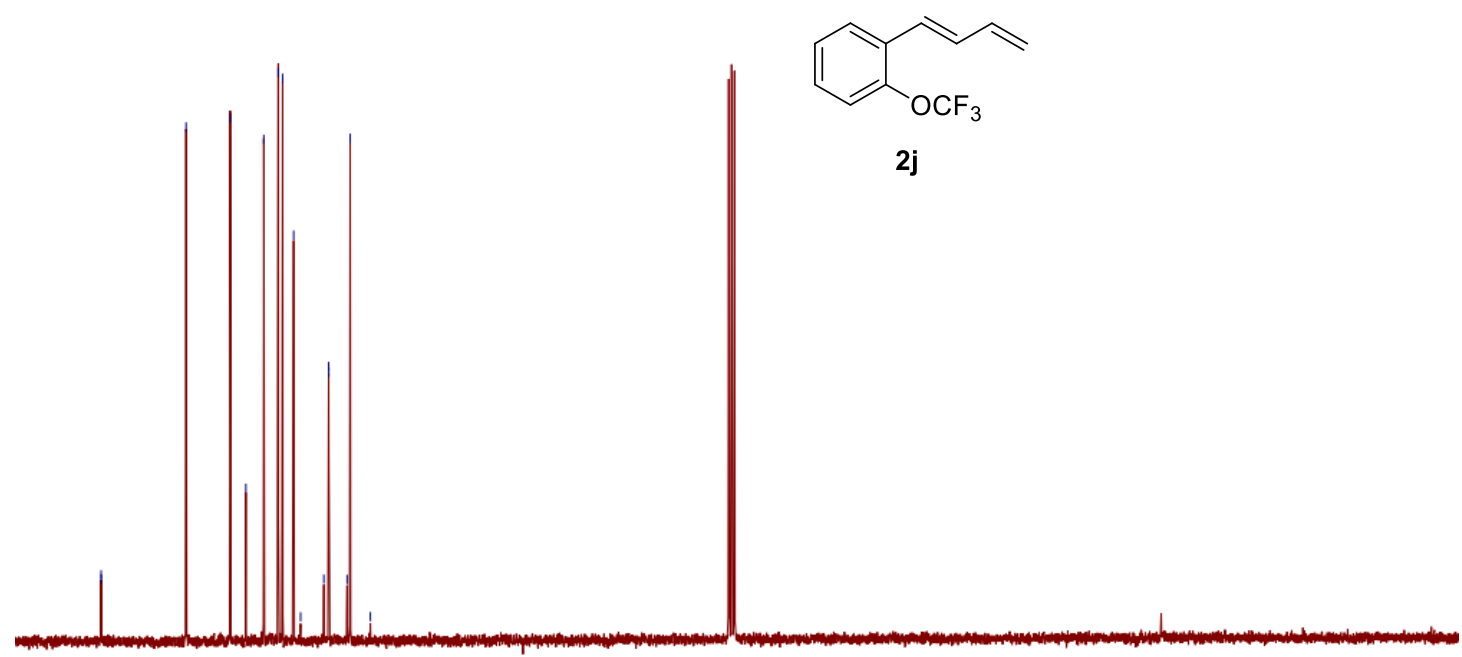

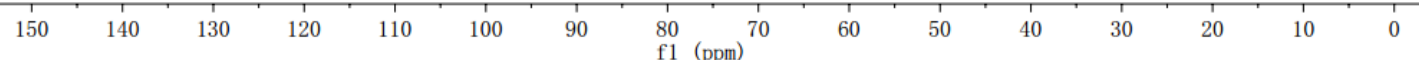


$2 n$

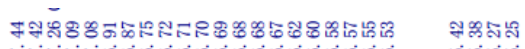

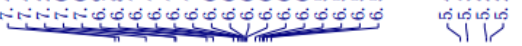

$1+1 / 2$

$\iint$

$\mathrm{MeO}$

$\mathrm{N}_{\mathrm{Br}} \mathrm{\sim}$

2n

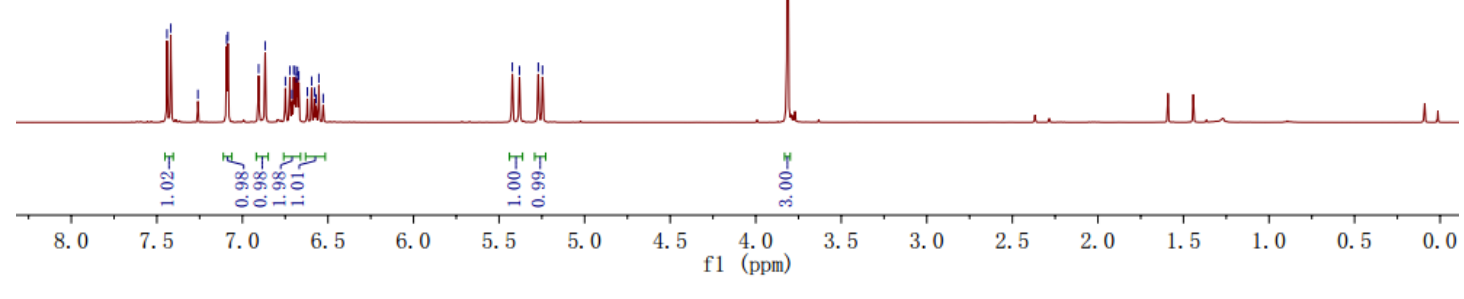

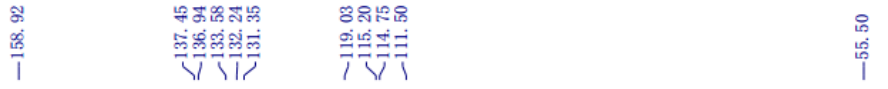

$\mathrm{C}_{\mathrm{Br}}^{\mathrm{MeO}}>$

$2 n$

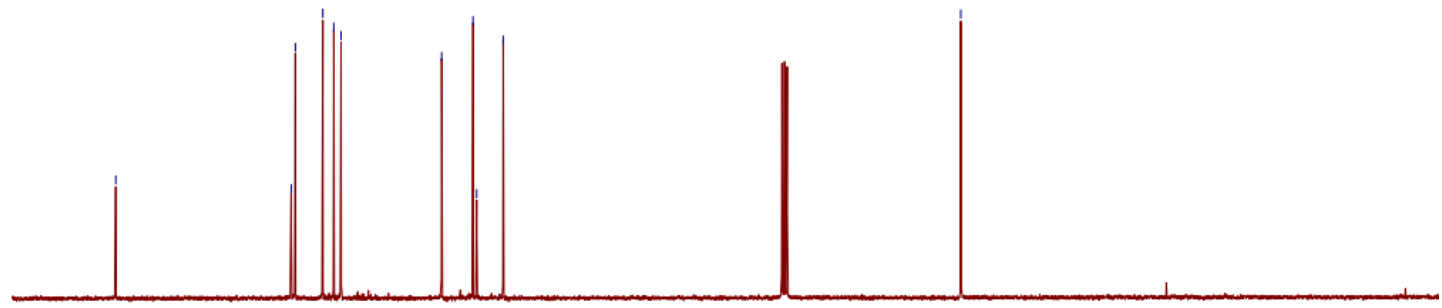

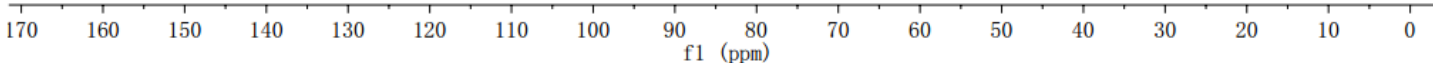


3 aa

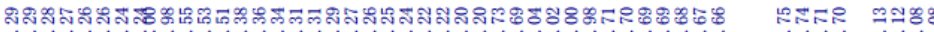

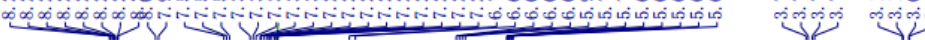
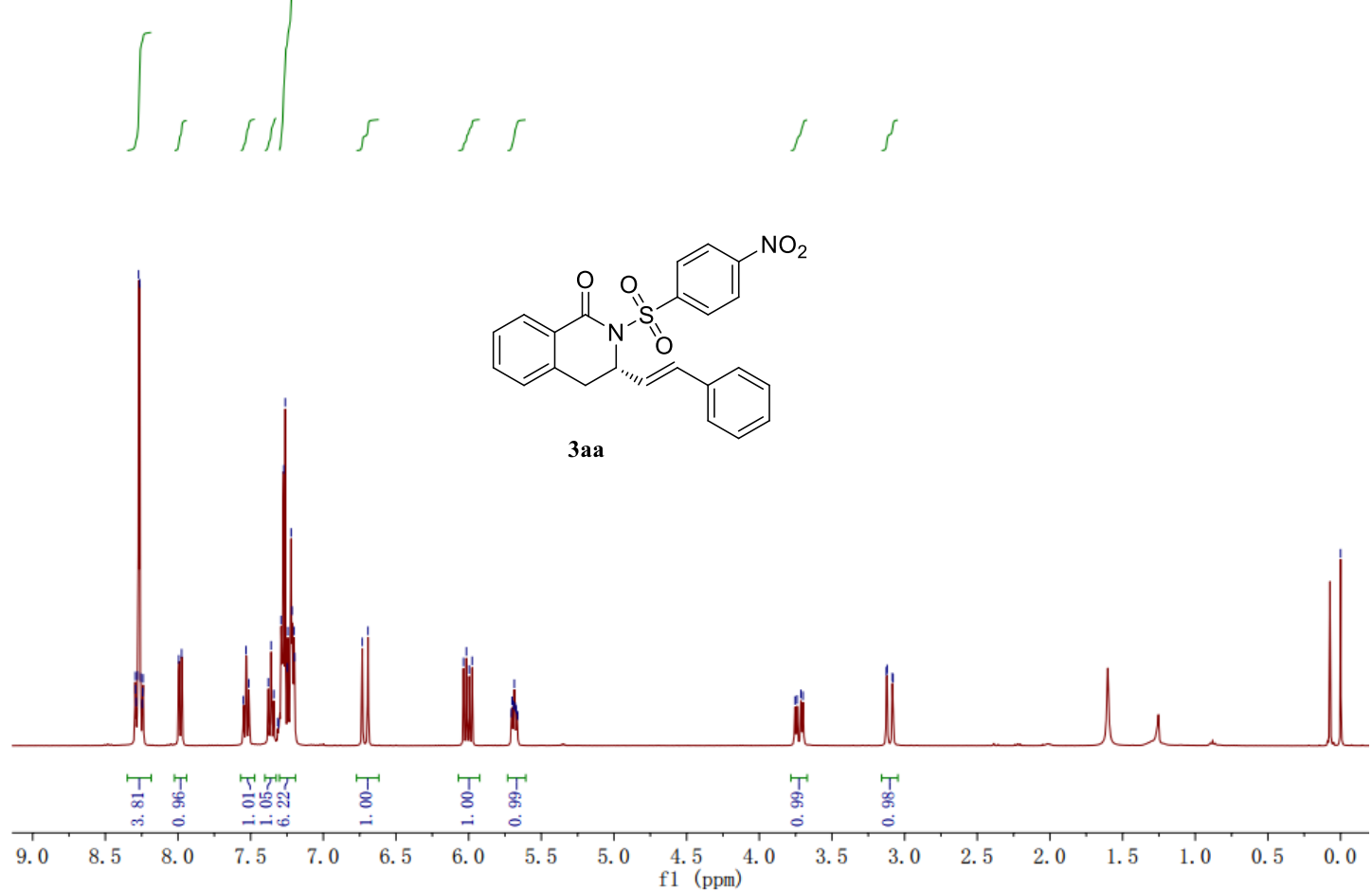

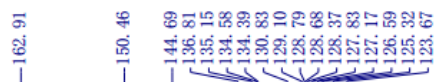
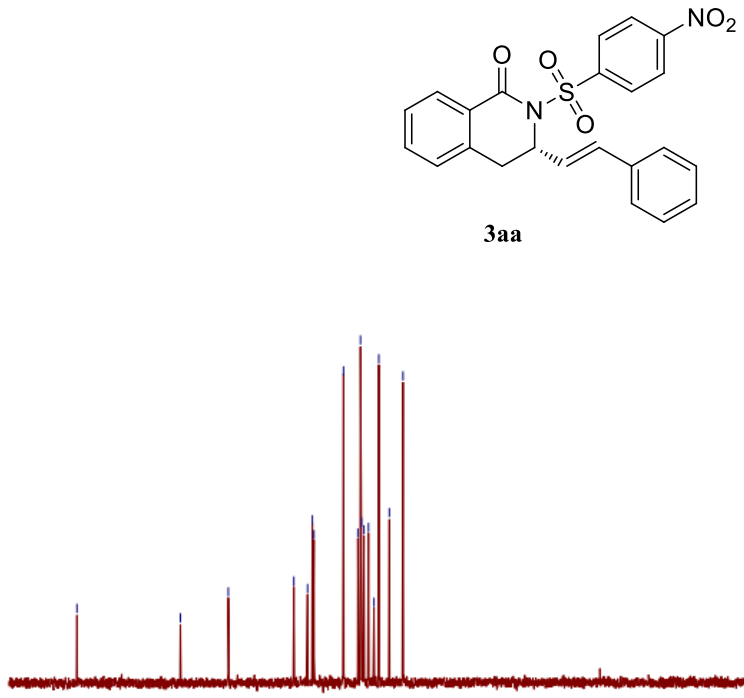

$\begin{array}{lllllllll}70 & 160 & 150 & 140 & 130 & 120 & 110 & 100 & 90\end{array}$ 


\section{3 ba}

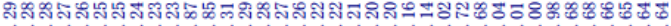

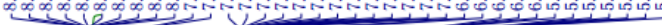

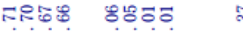

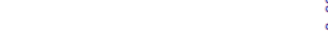
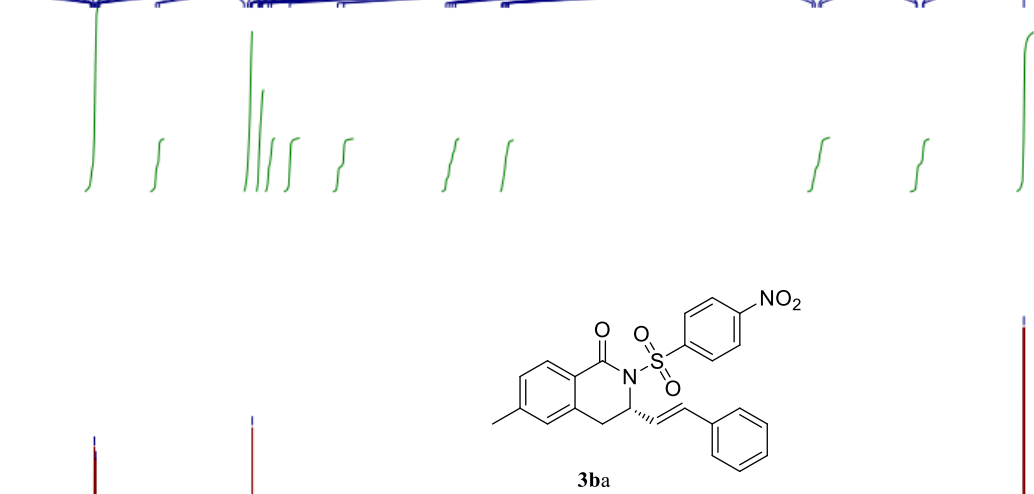

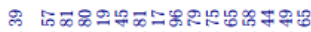

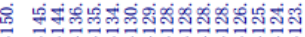

$\begin{array}{lll}\infty & \infty & 9 \\ \infty & \dot{0} & + \\ 0 & i & T\end{array}$
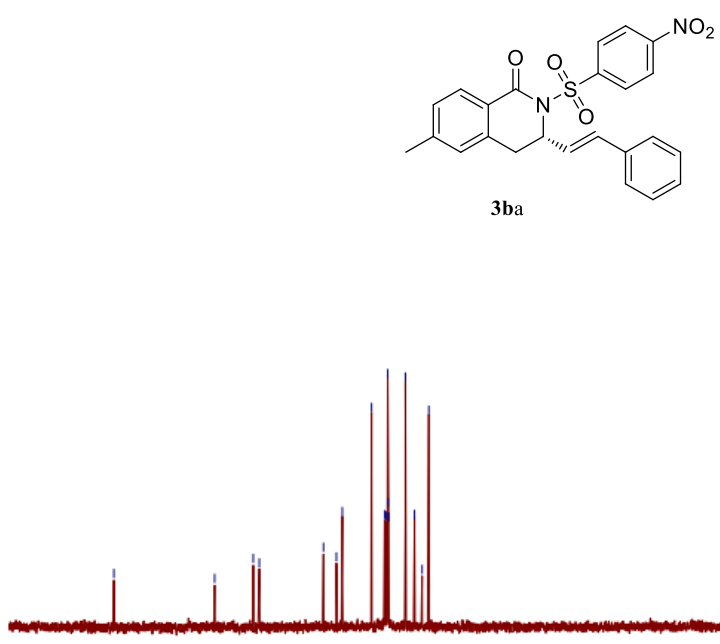

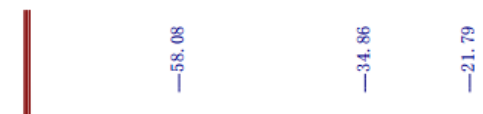

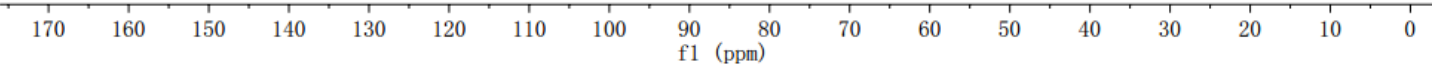


3 ca

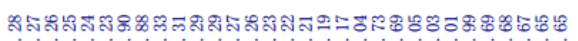

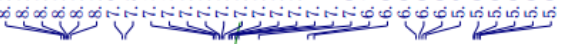

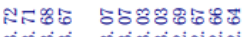

ำลำ

8
1
1
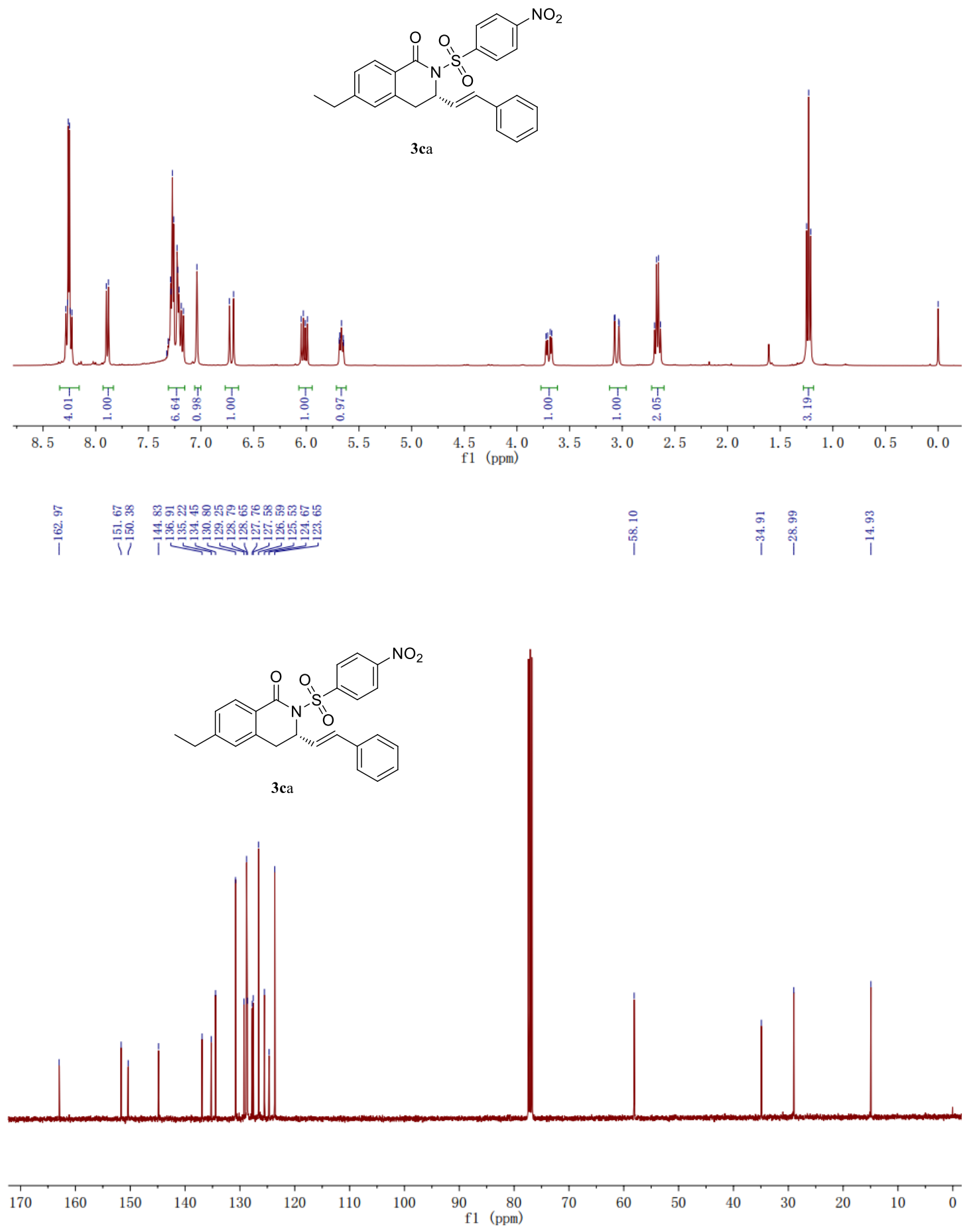

S14 


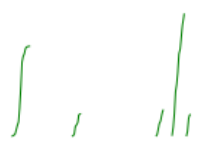<smiles>CC(C)(C)c1ccc2c(c1)CC(C=Cc1ccccc1)N(S(=O)(=O)c1ccc([N+](=O)[O-])cc1)C2=O</smiles>

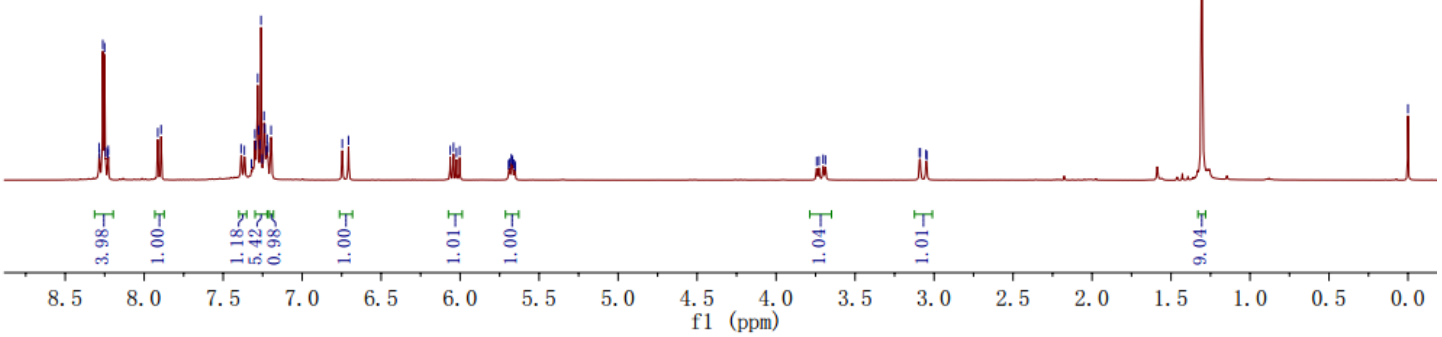

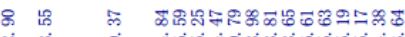

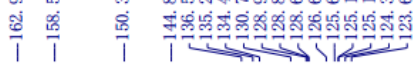

3da

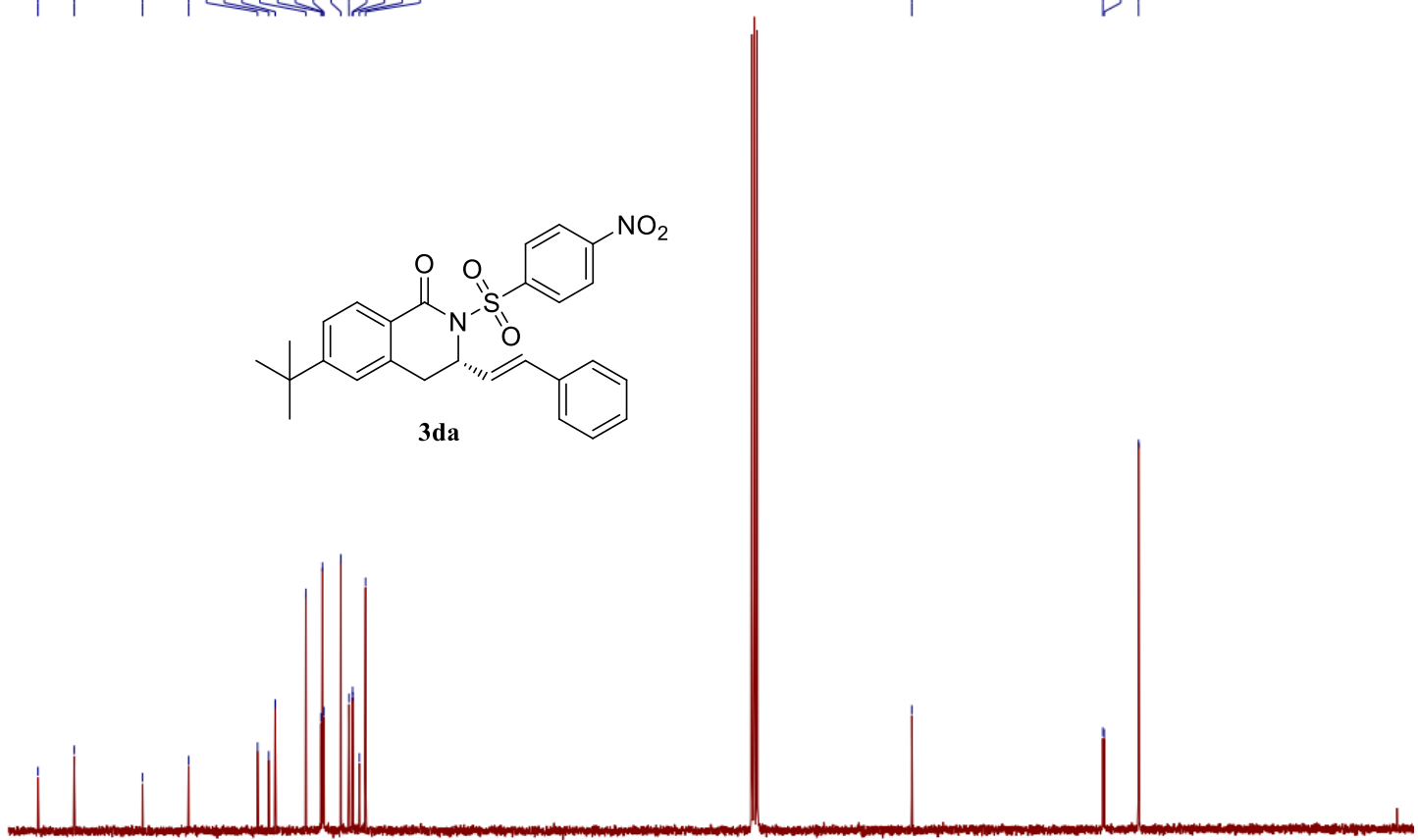

$\begin{array}{lllllllll}160 & 150 & 140 & 130 & 120 & 110 & 100 & 90 & 80 \\ \mathrm{f} 1 & \begin{array}{l}80 \\ (\mathrm{ppm})\end{array}\end{array}$ 


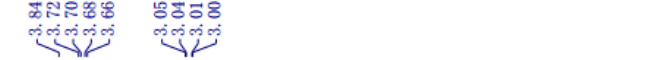
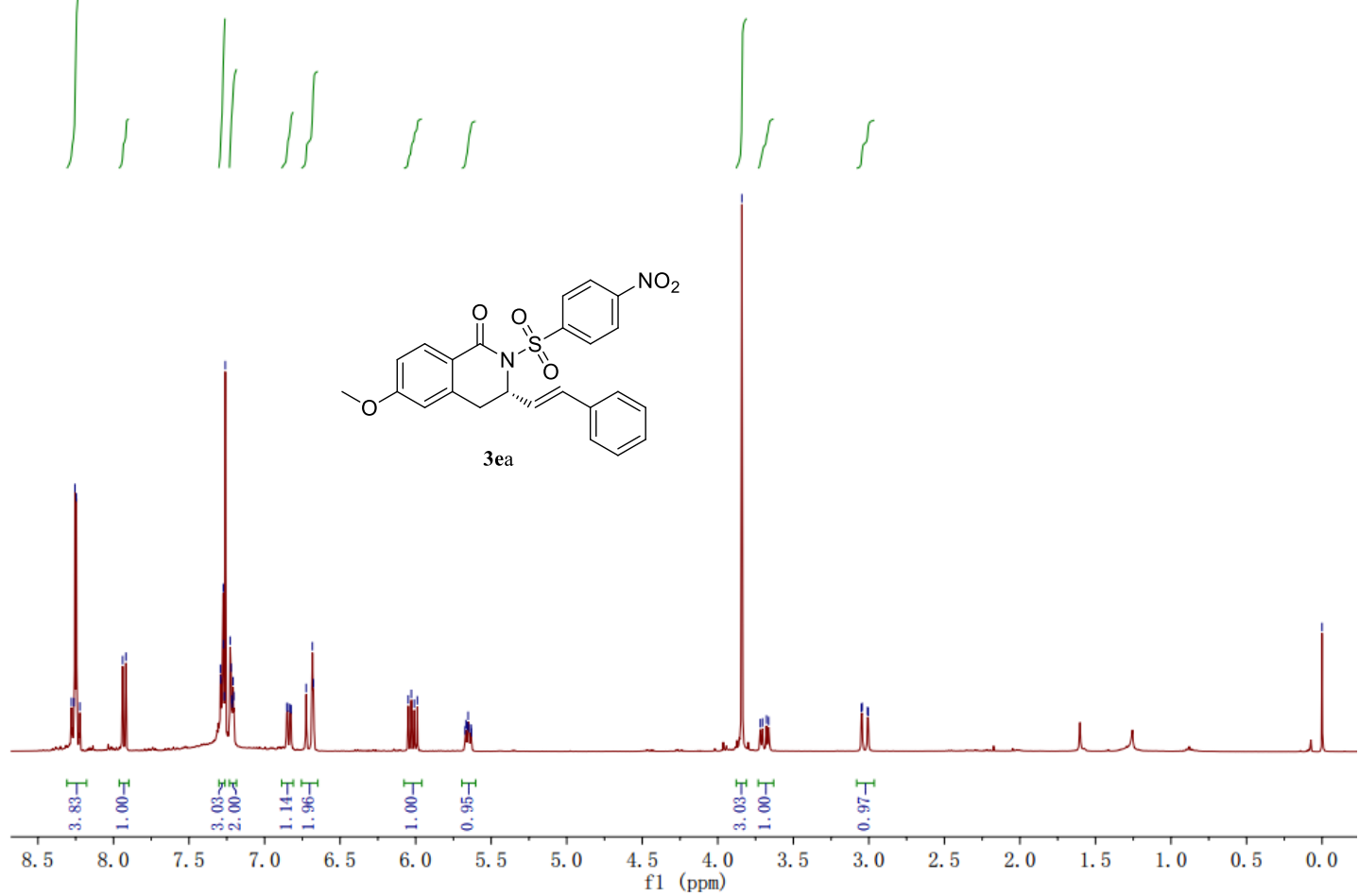

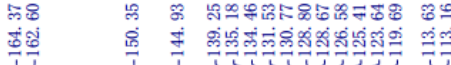

(1)

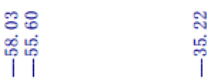

i

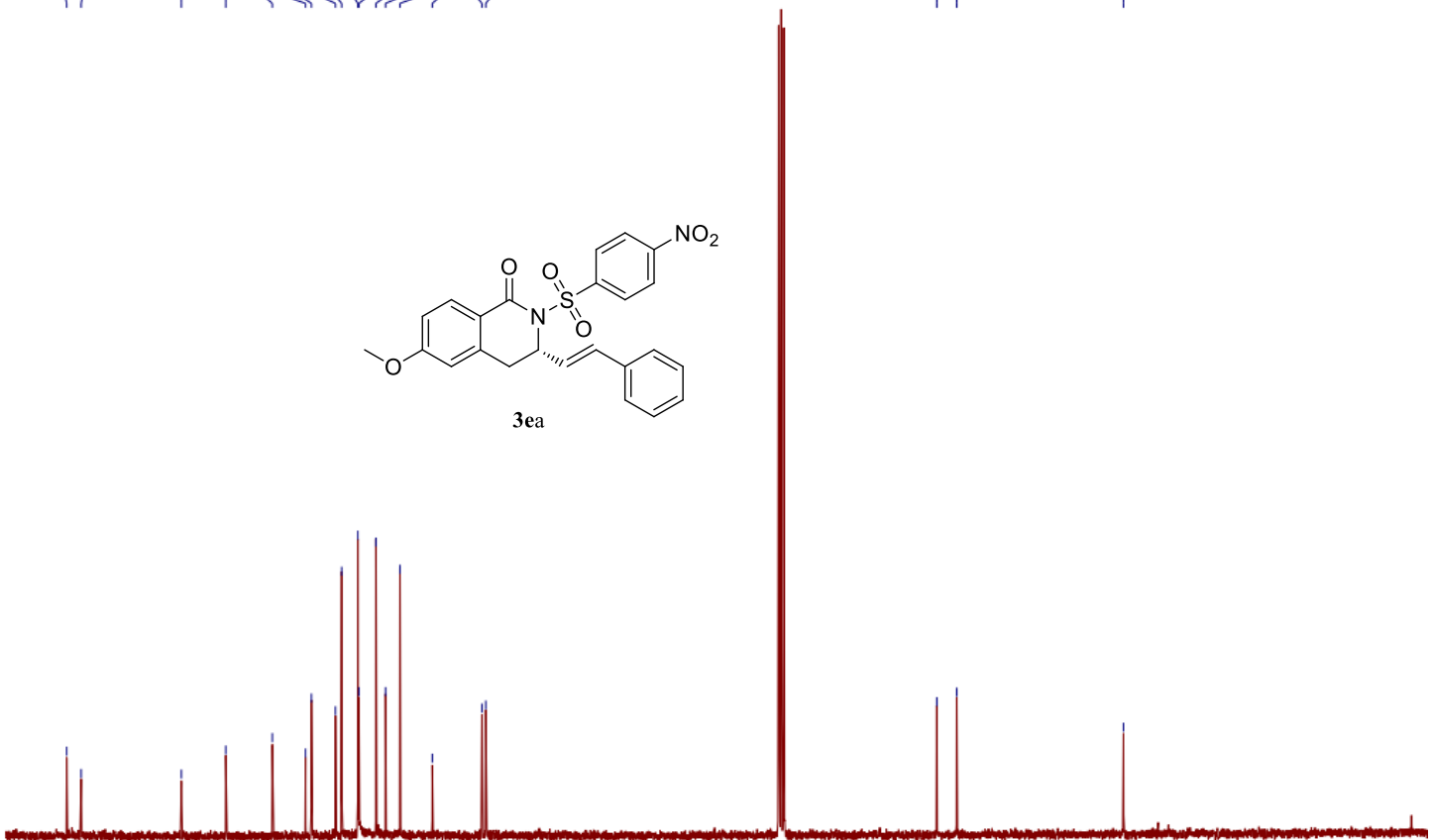

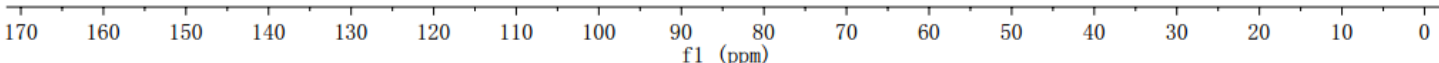



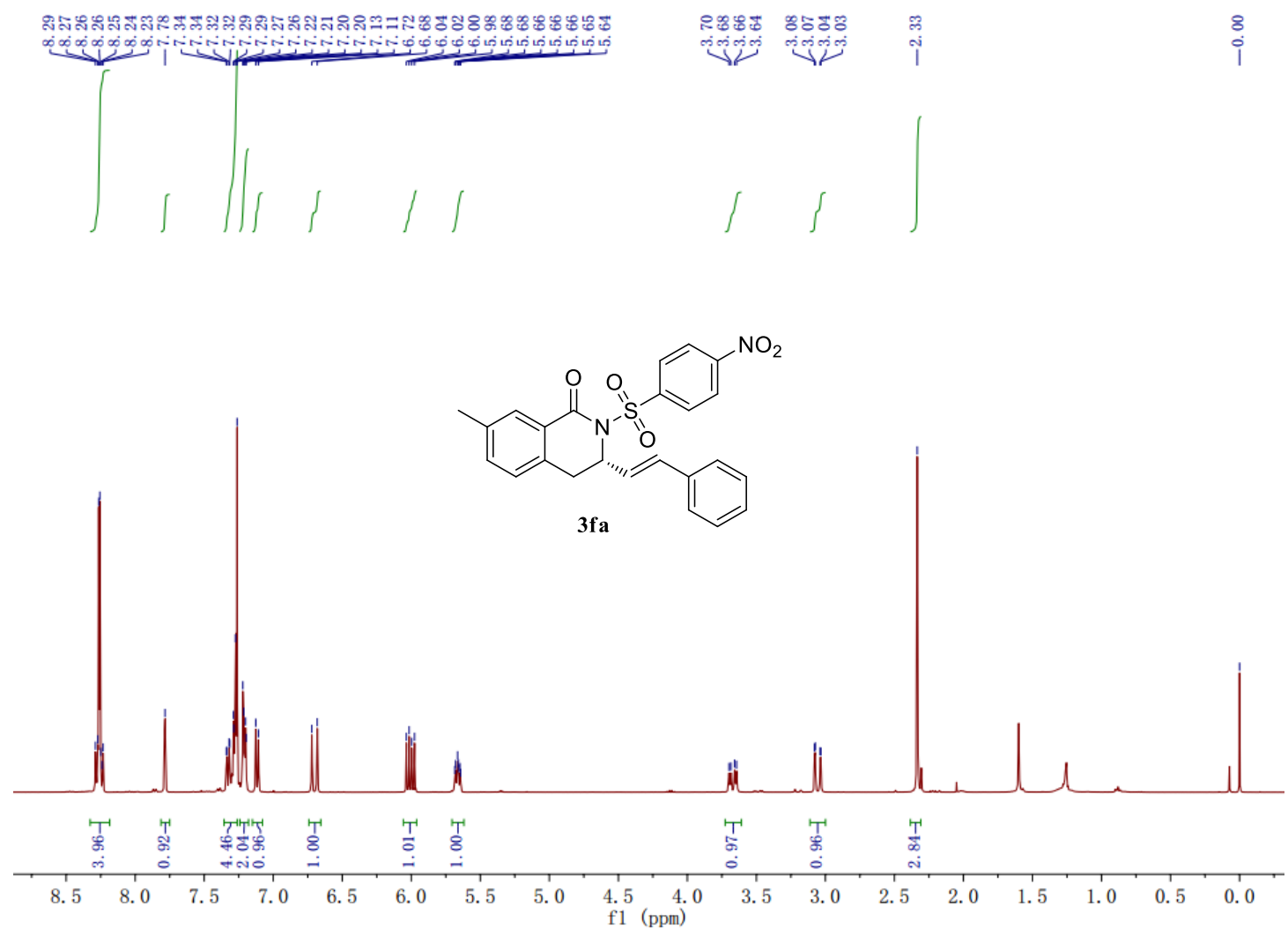

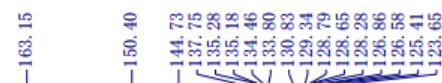
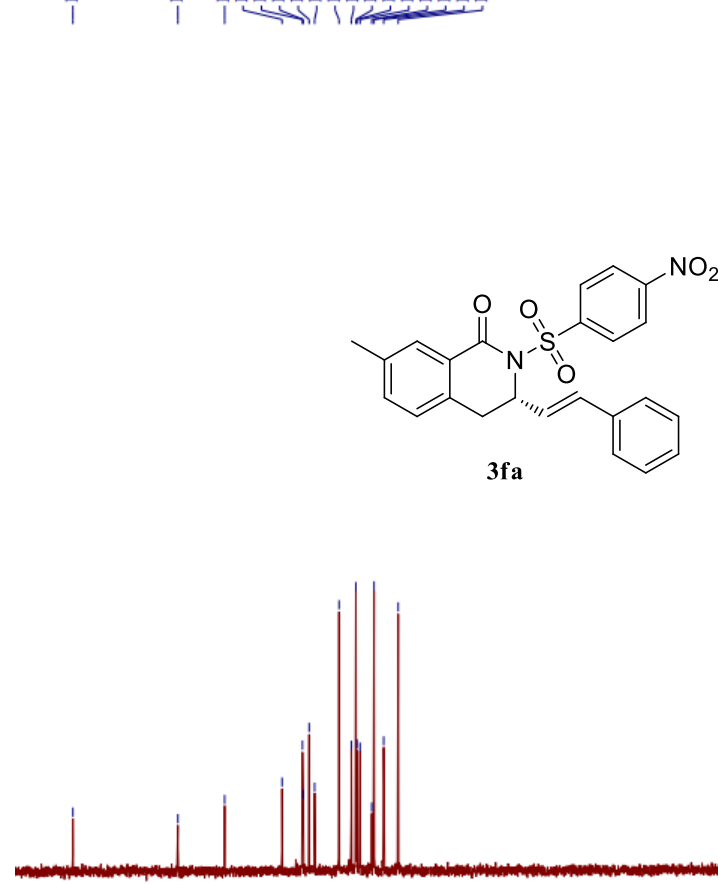

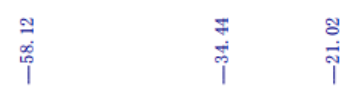

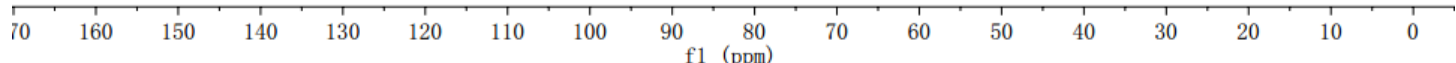


3ga

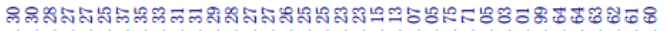

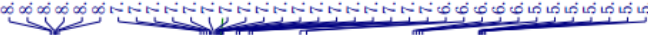

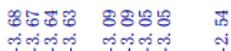

8
$i$
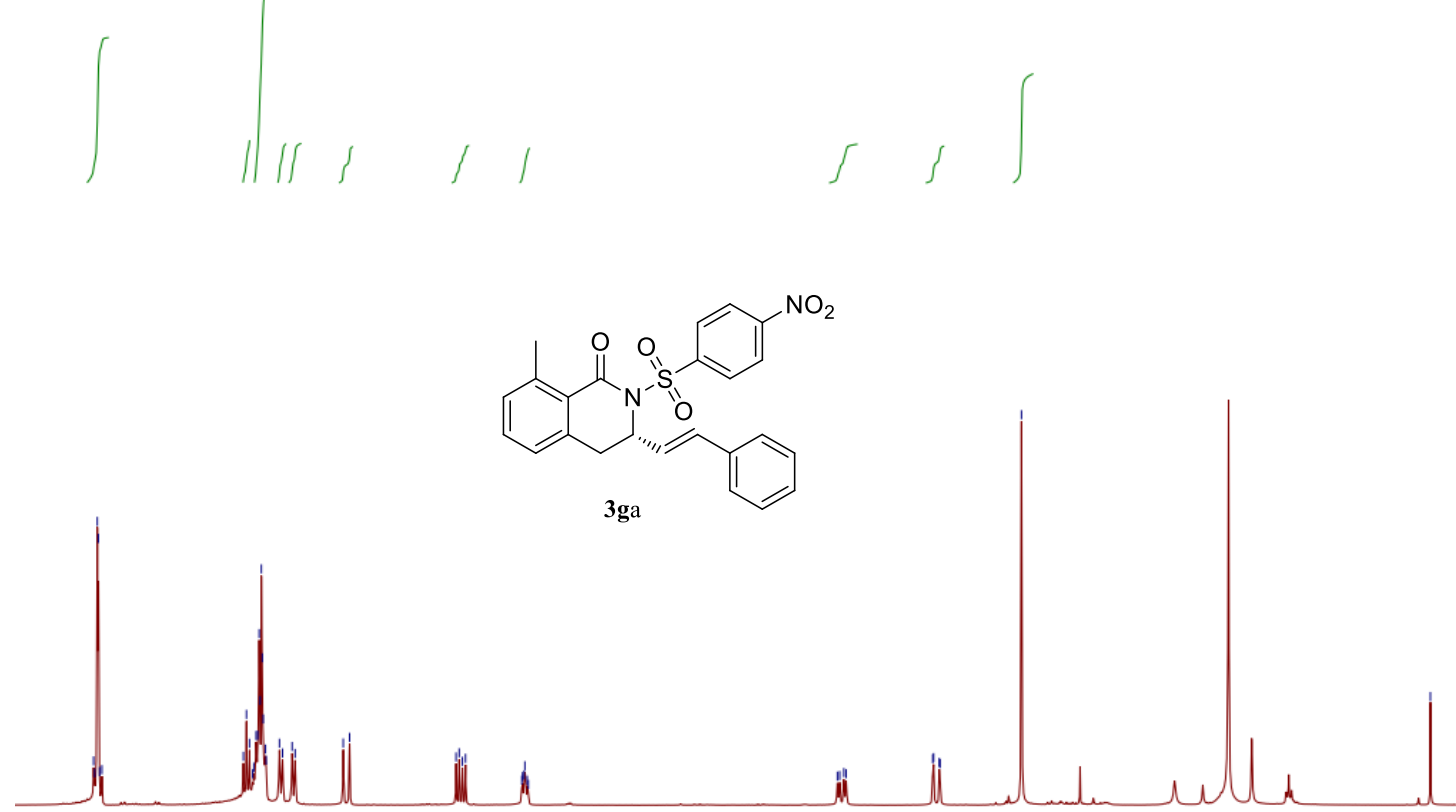

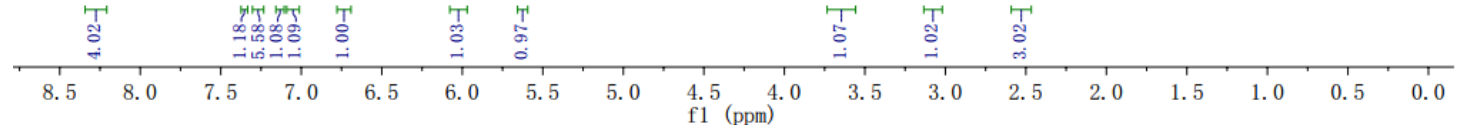

เ

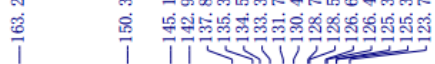

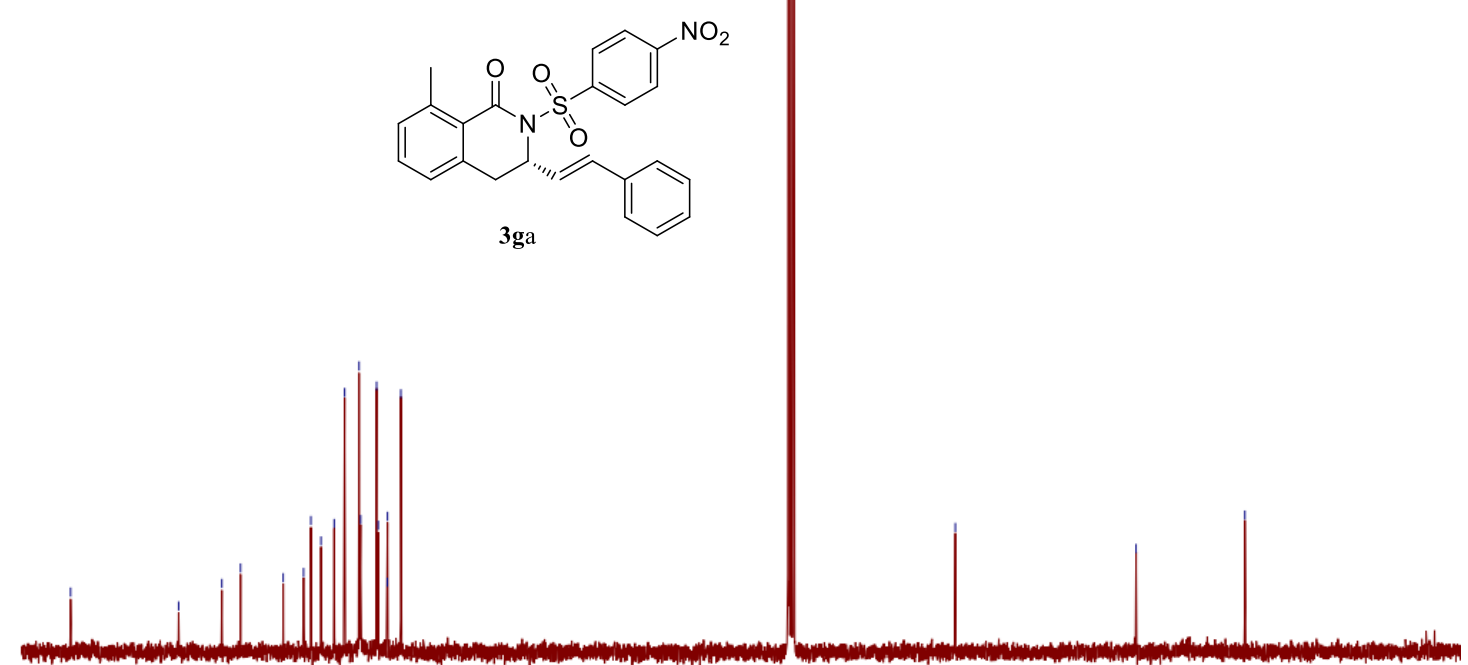

$\begin{array}{lllllllll}160 & 150 & 140 & 130 & 120 & 110 & 100 & 90 & 80\end{array}$

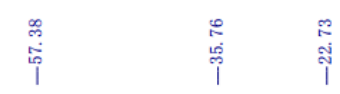




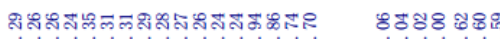

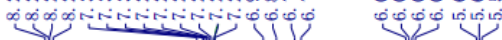

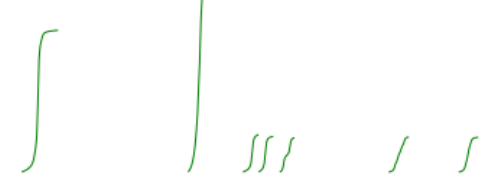

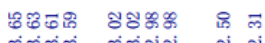

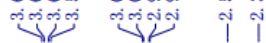

8
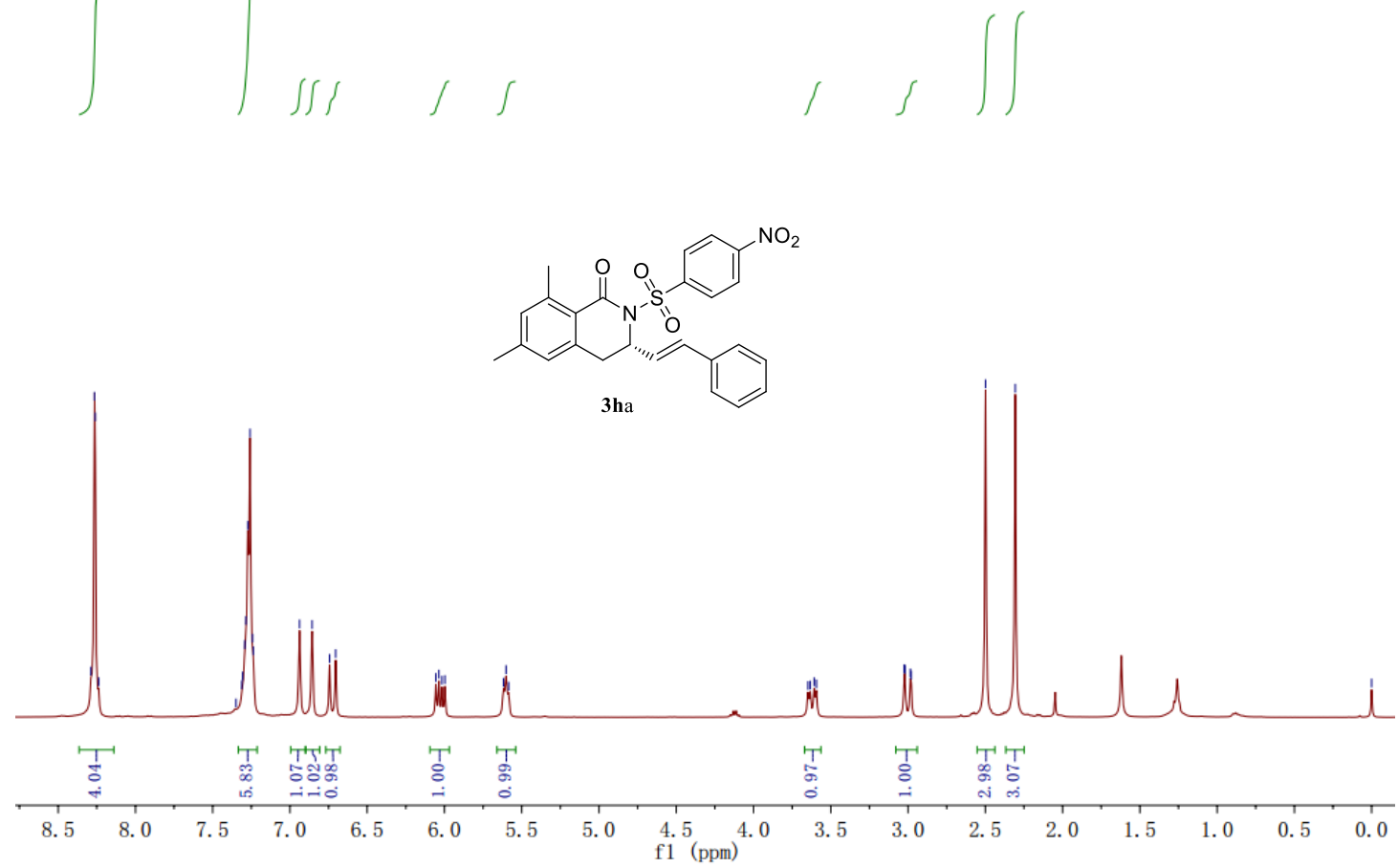

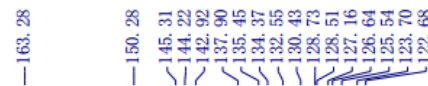

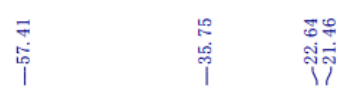
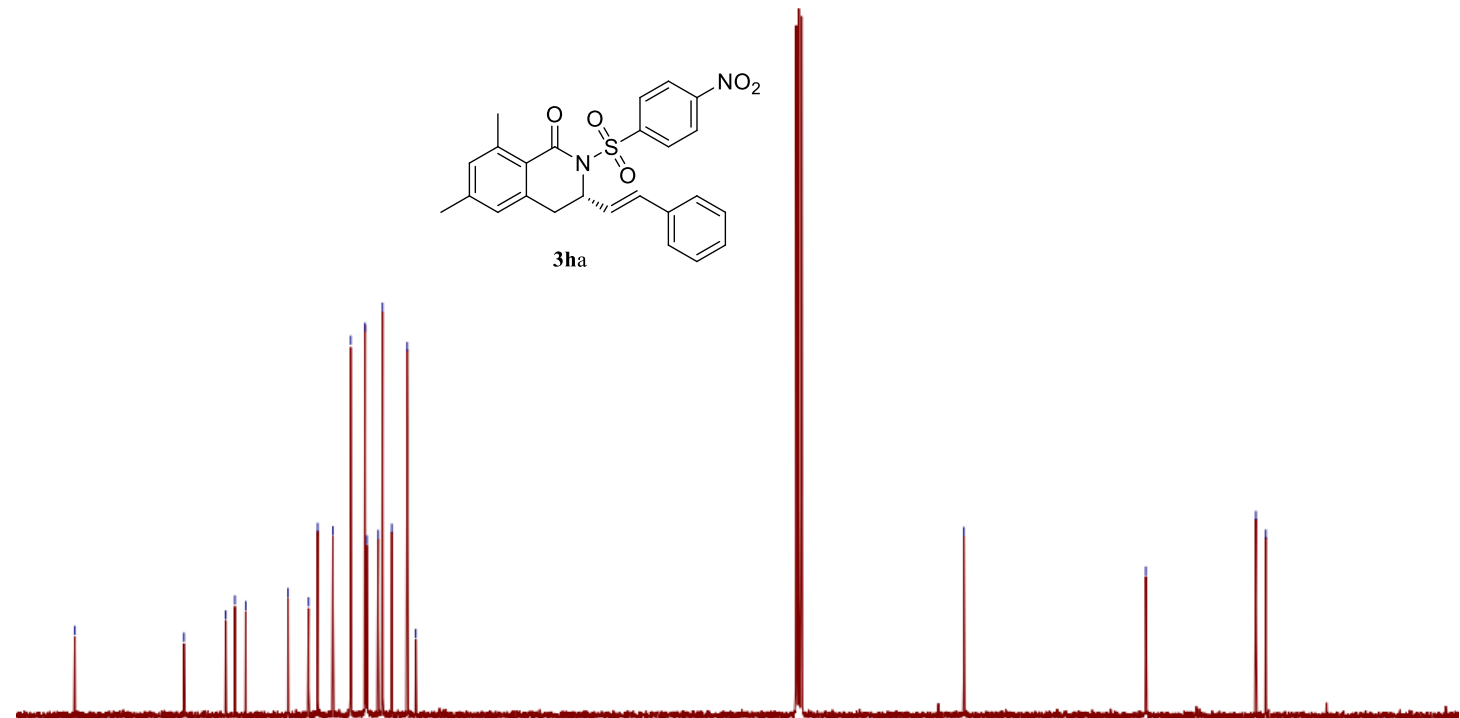

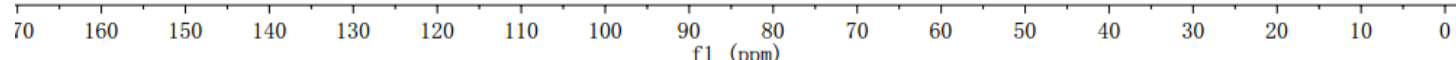


3 ia
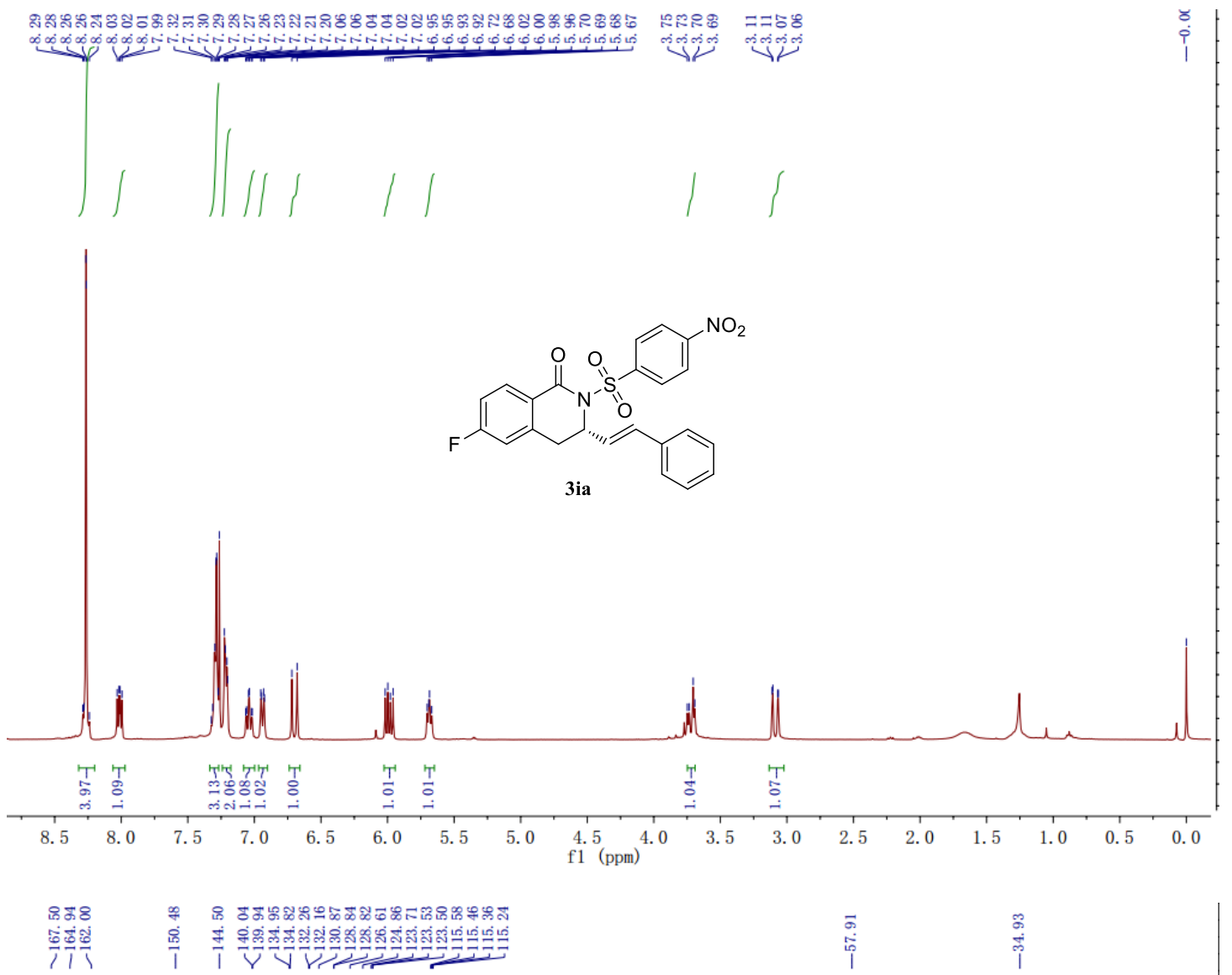

a

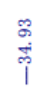

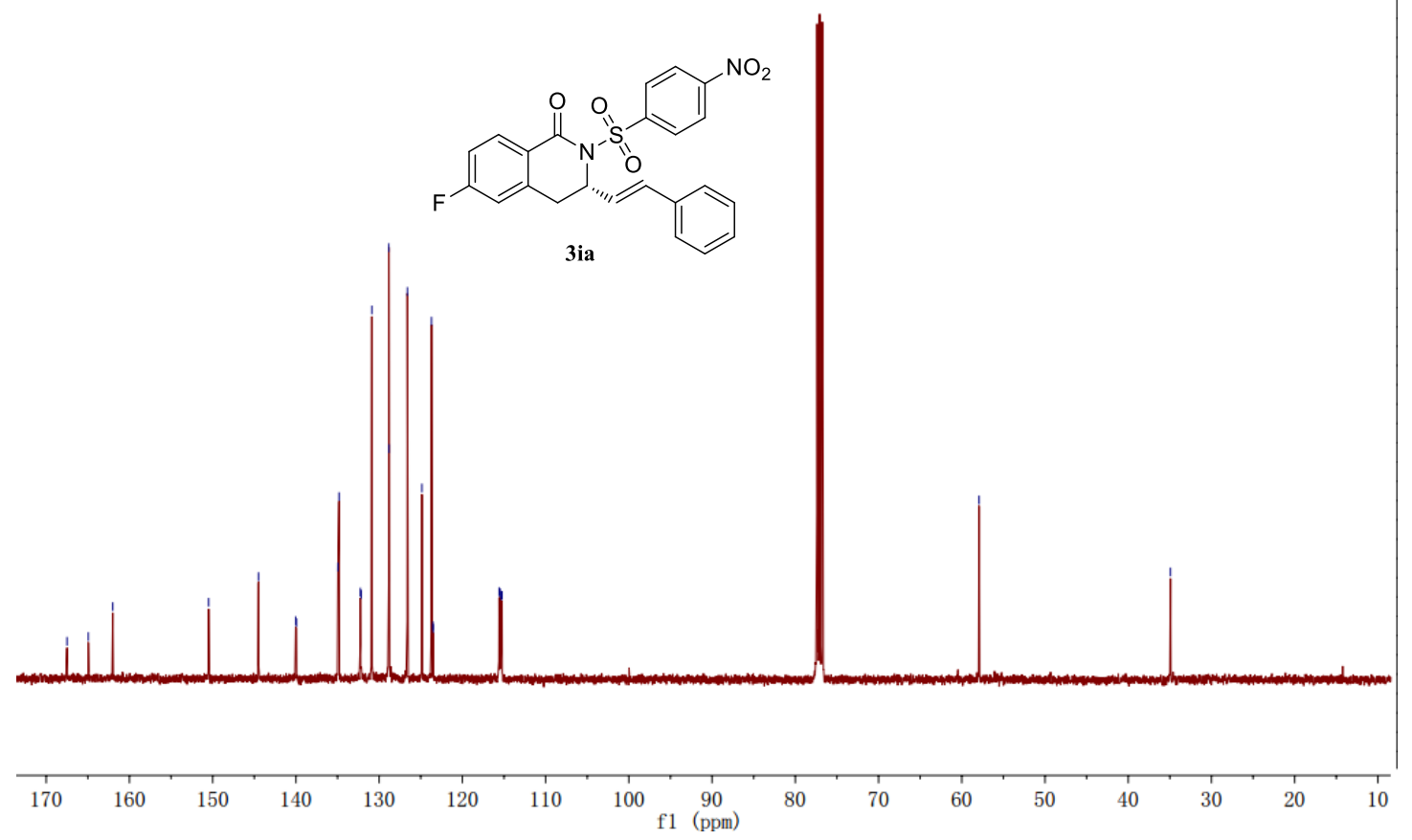

S20 
$3 \mathbf{j a}$

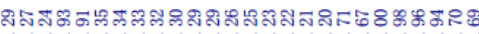

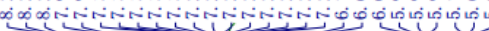

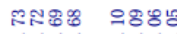

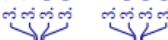

8
$i$

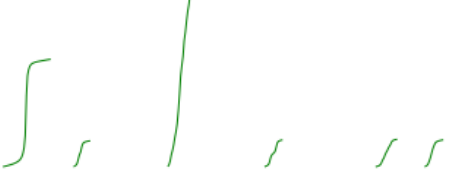<smiles>O=C1c2ccc(Cl)cc2C(C=Cc2ccccc2)CN1S(=O)(=O)c1ccc([N+](=O)[O-])cc1</smiles>
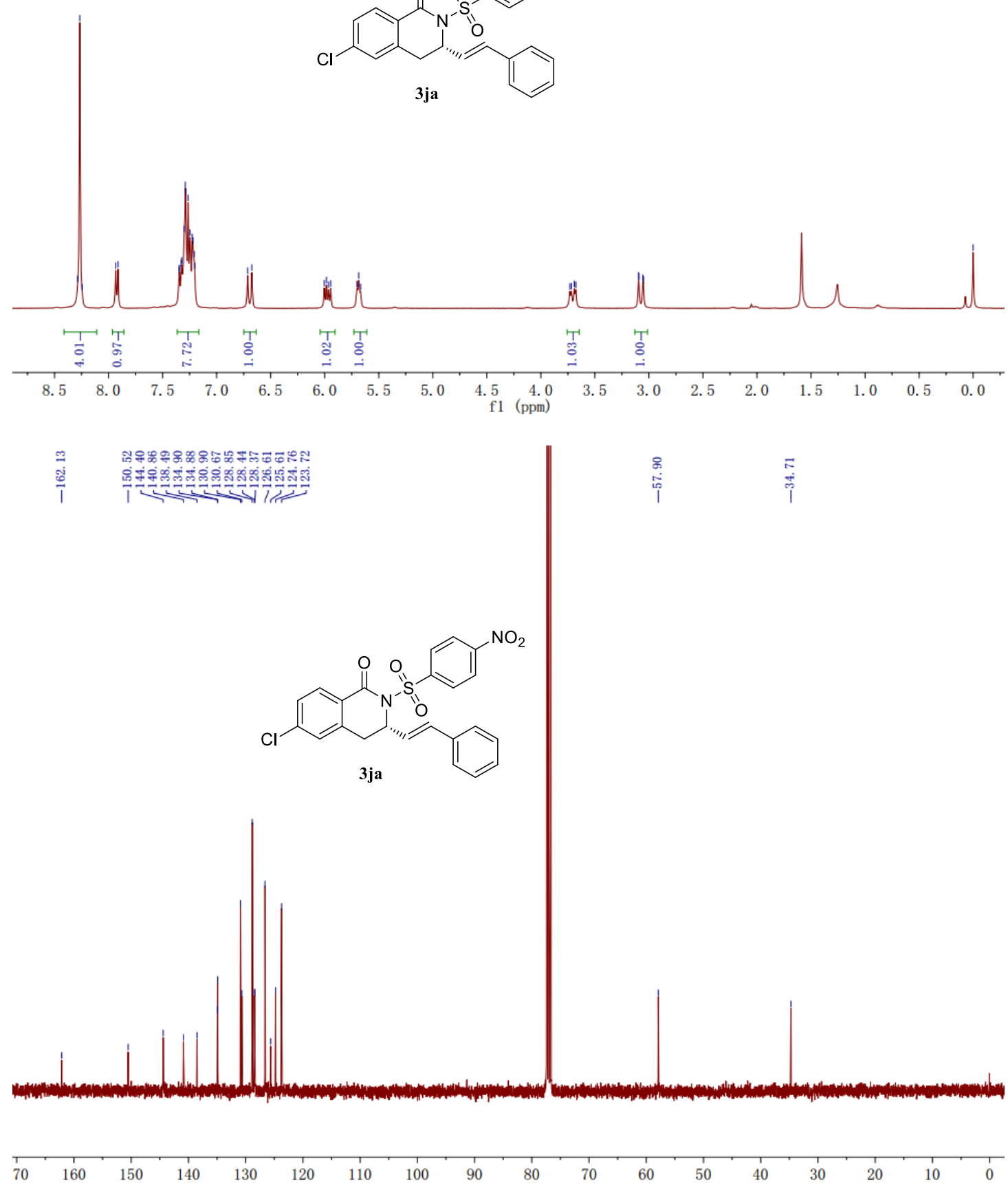

f1 (ppm) 
$3 \mathrm{ka}$

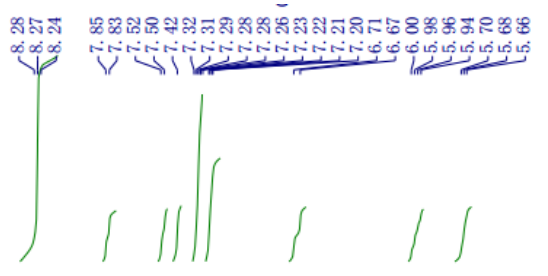

Nํ요

8
$\vdots$
1
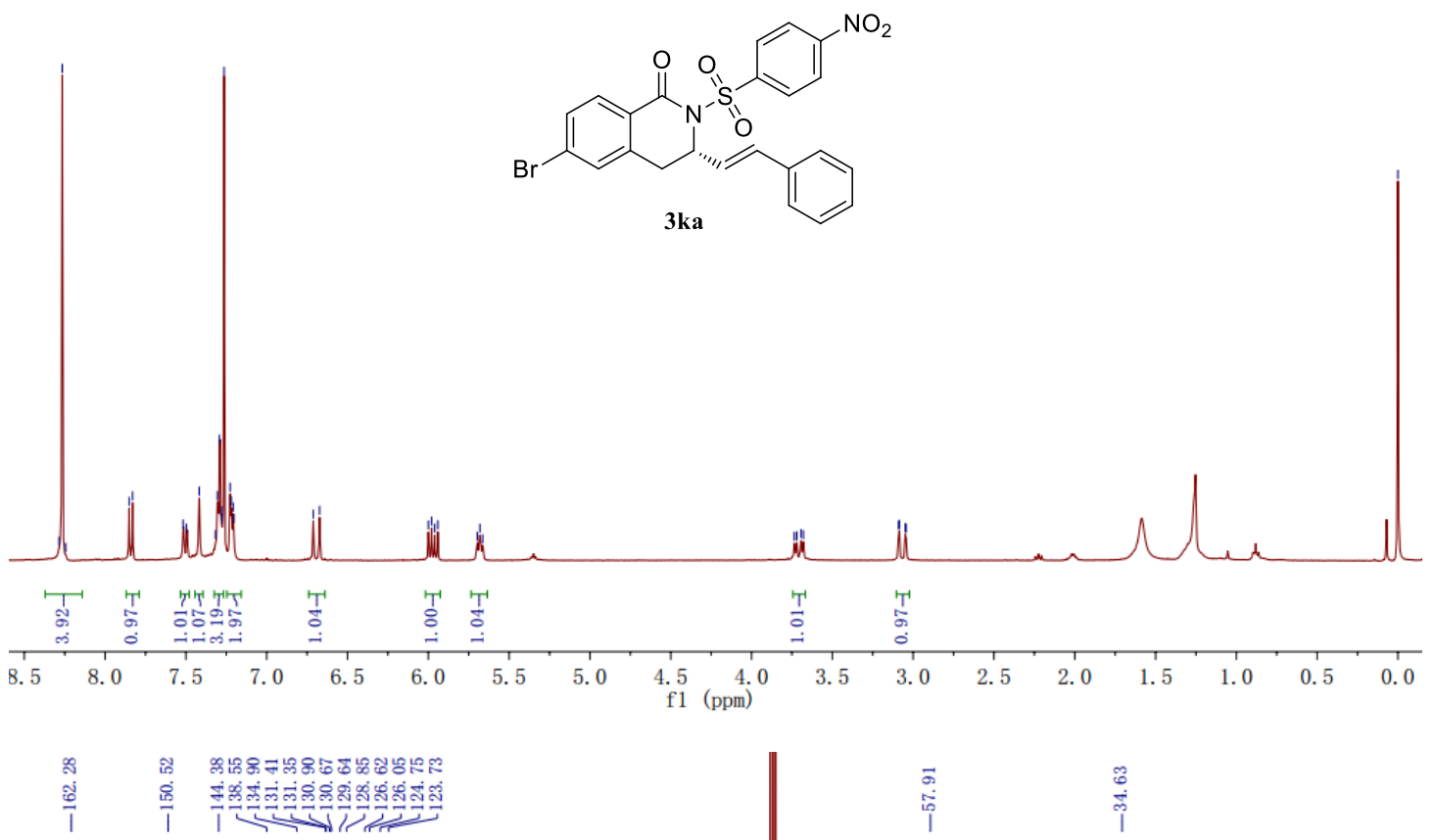

它

:
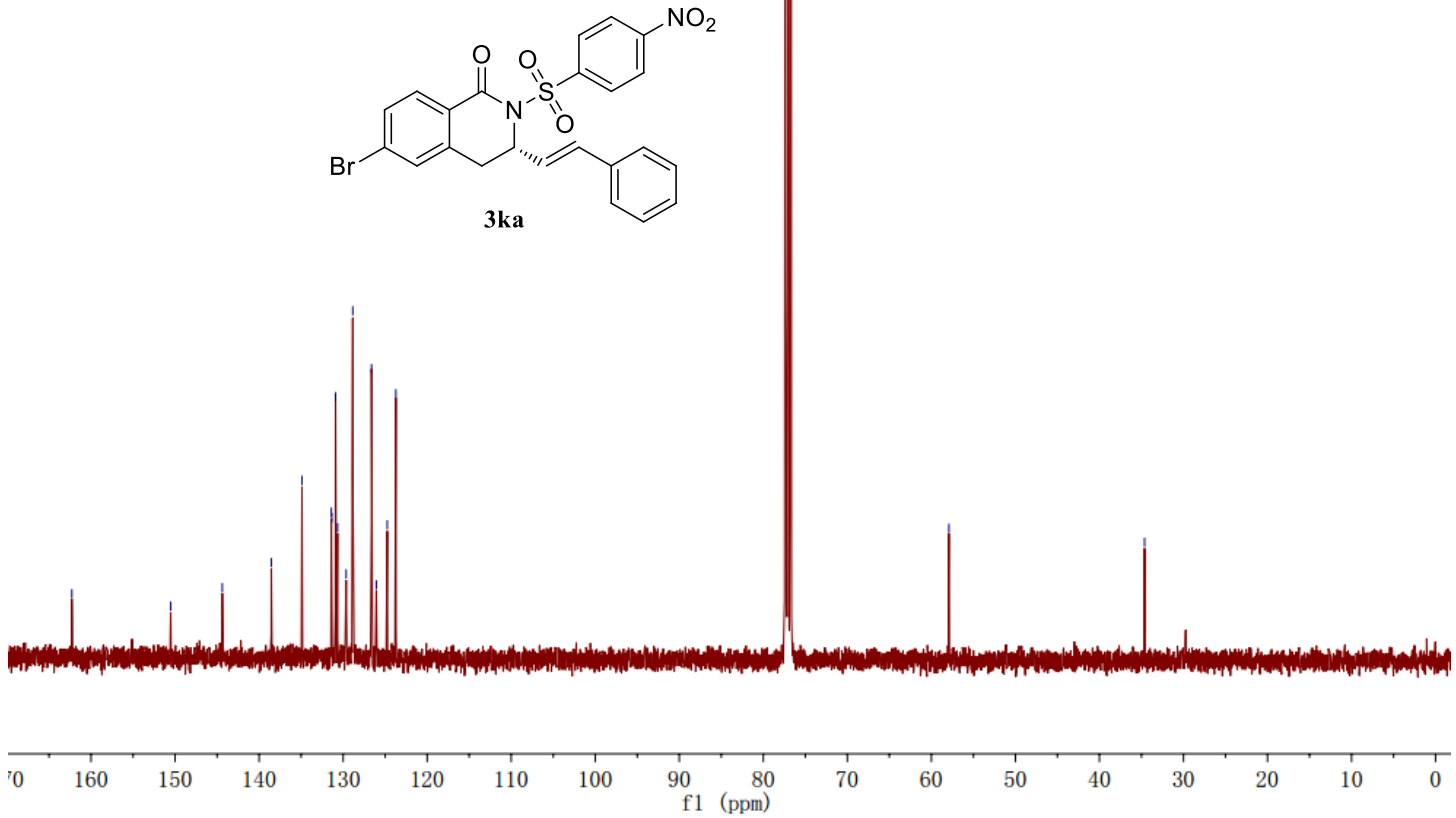

S22 
31 a

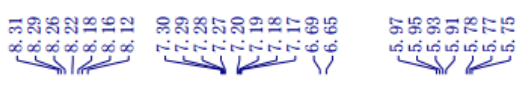

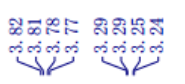

8
1
1
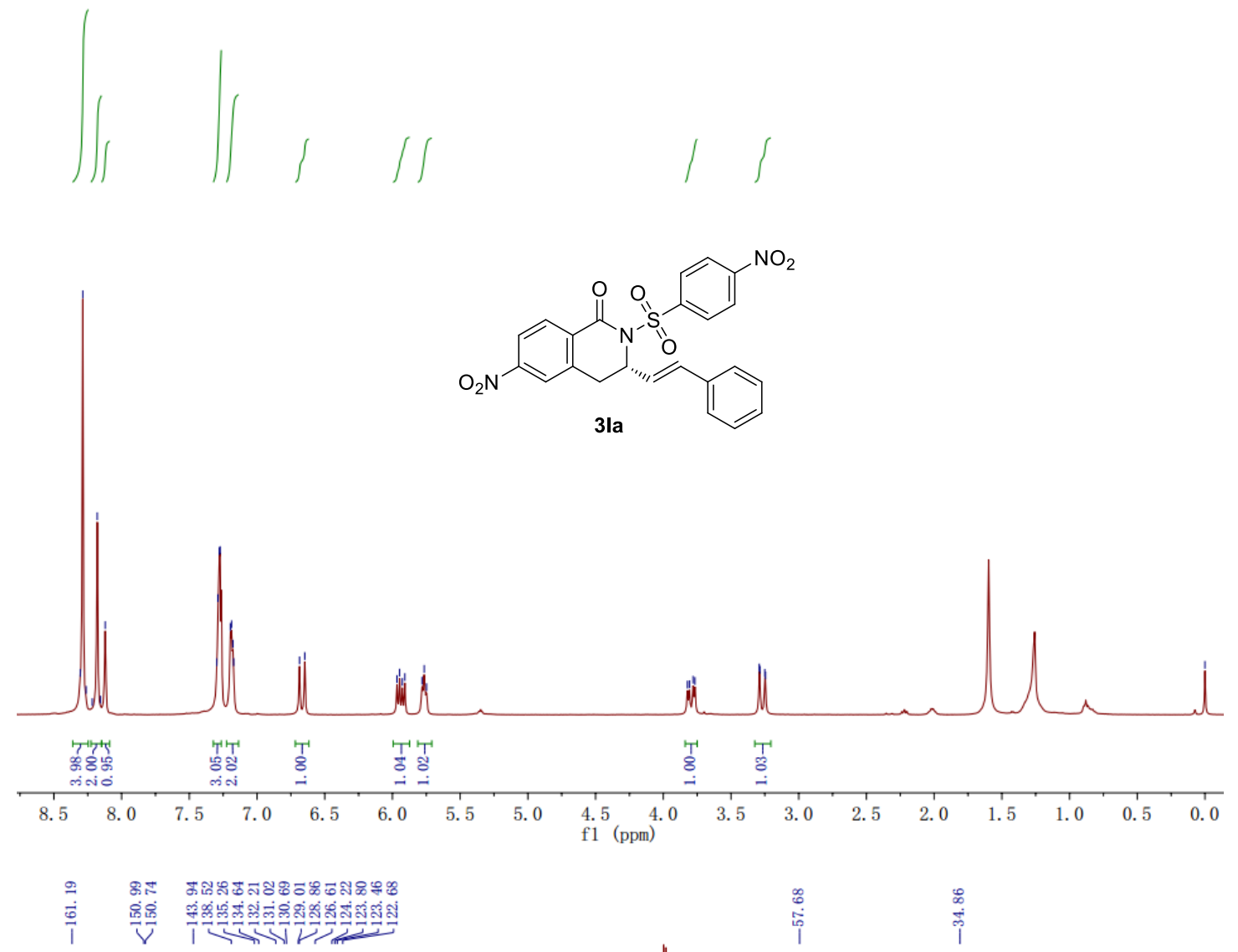

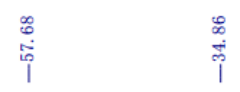

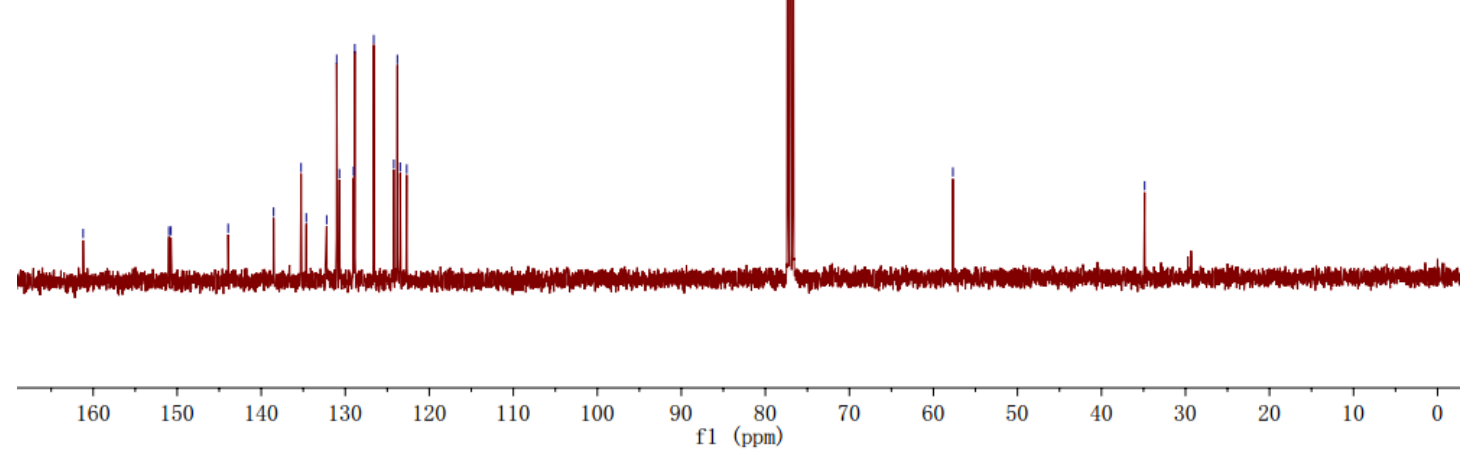

S23 


\section{$3 \mathrm{ma}$}

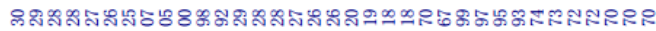

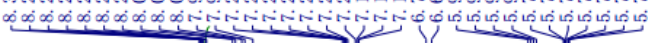

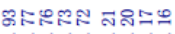

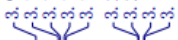
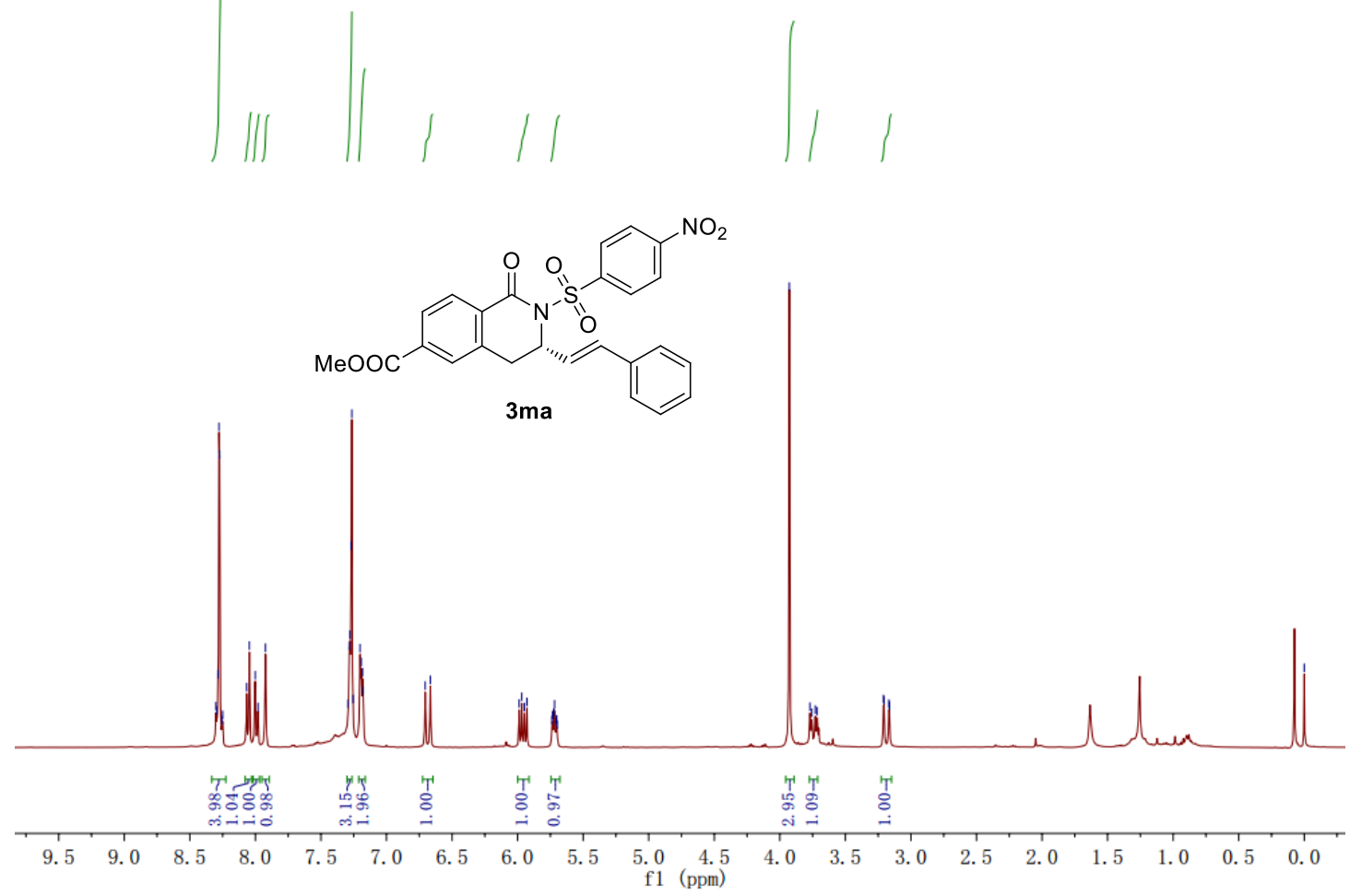

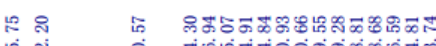

iी

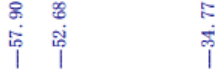<smiles>COC(=O)c1ccc2c(c1)C[C@H](/C=C/c1ccccc1)N(S(=O)(=O)c1ccc([N+](=O)[O-])cc1)C2=O</smiles>

170

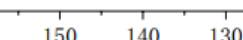

120

$90 \quad 80$

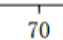

50

$\begin{array}{llll}1 & 1 & 1 & 1 \\ 30 & 20 & 10 & 0\end{array}$ 


\section{3na}

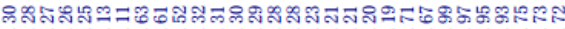
$\underbrace{\infty} \operatorname{lin}^{\infty} 0^{\infty} \underbrace{\circ}$

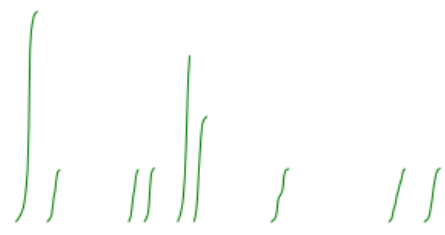

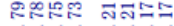

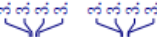

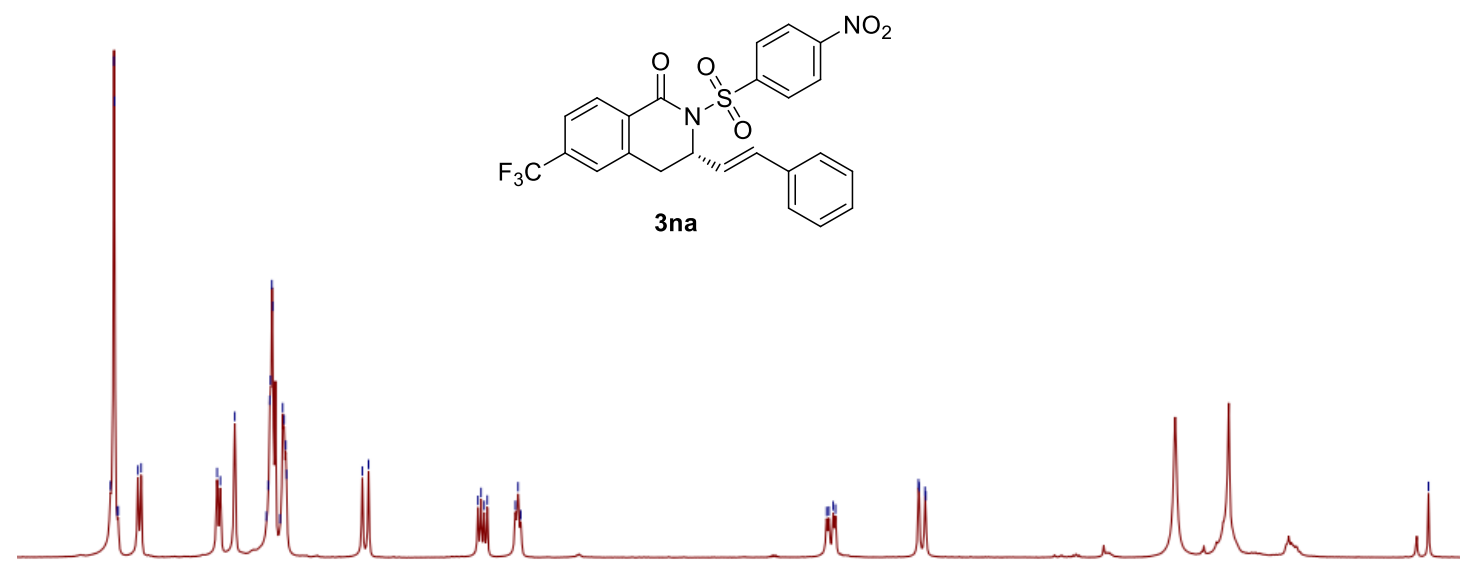

3na<smiles>CCCCC</smiles>

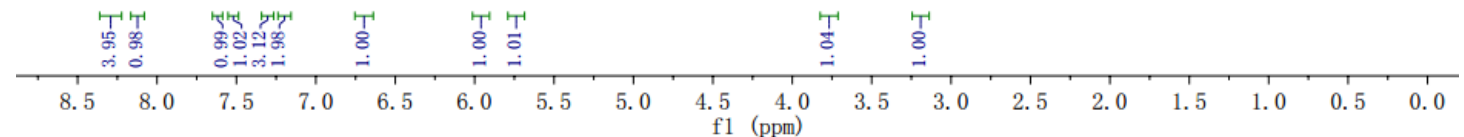

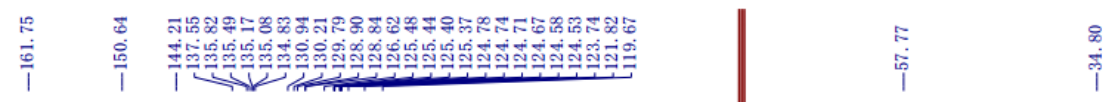<smiles>O=C1c2ccc(C(F)(F)F)cc2C[C@H](/C=C/c2ccccc2)N1S(=O)(=O)c1ccc([N+](=O)[O-])cc1</smiles>

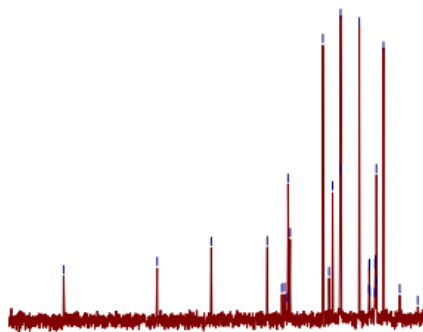

$\begin{array}{llllllll}160 & 150 & 140 & 130 & 120 & 110 & 100 & 90\end{array}$ 


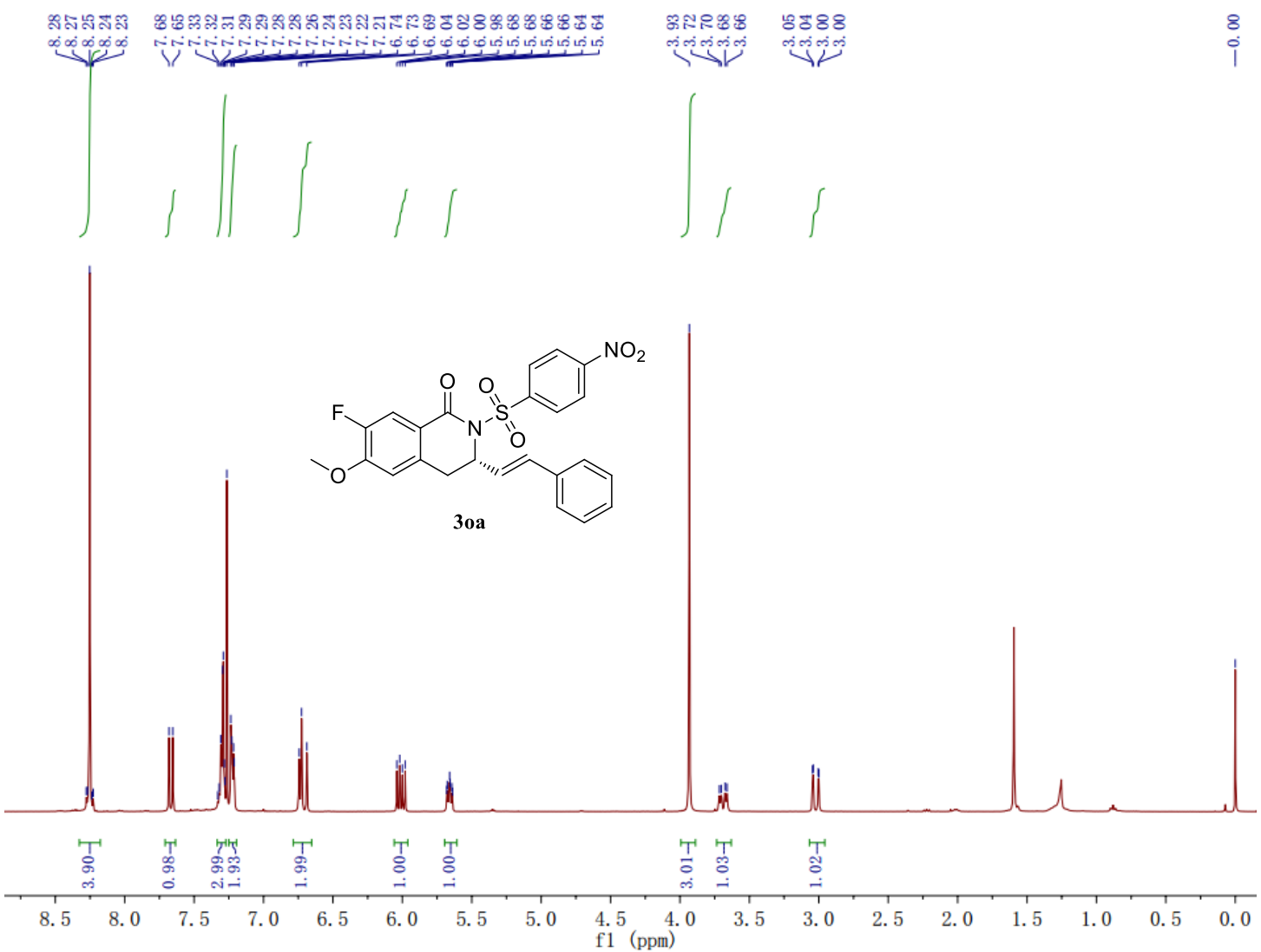

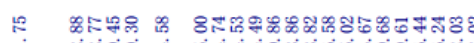

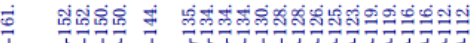

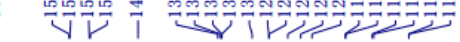
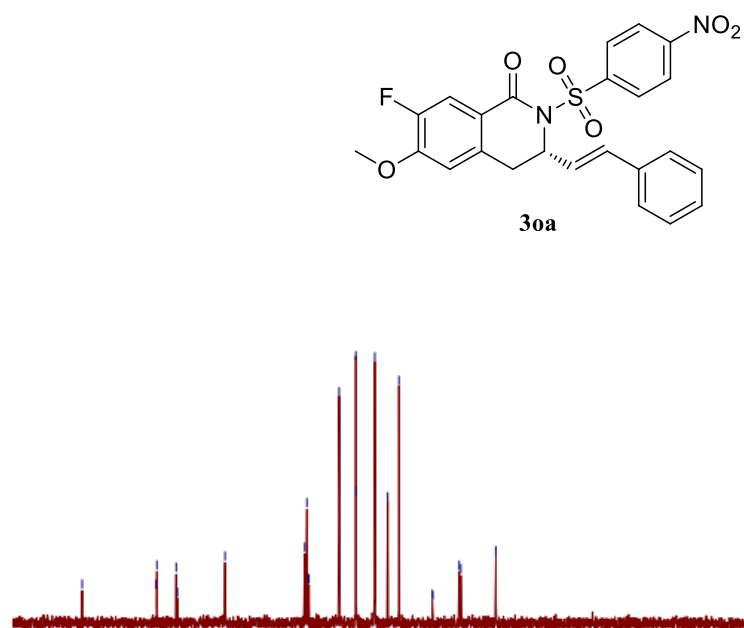

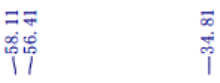

\begin{tabular}{rrrrrrrrrr}
\hline 70 & 160 & 150 & 140 & 130 & 120 & 110 & 100 & 90 & 80 \\
$\mathrm{f} 1$ & $(\mathrm{ppm})$
\end{tabular} 
$3 a b$
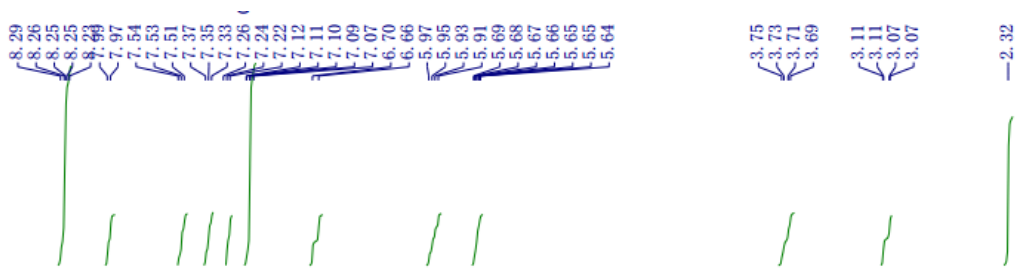

8
0
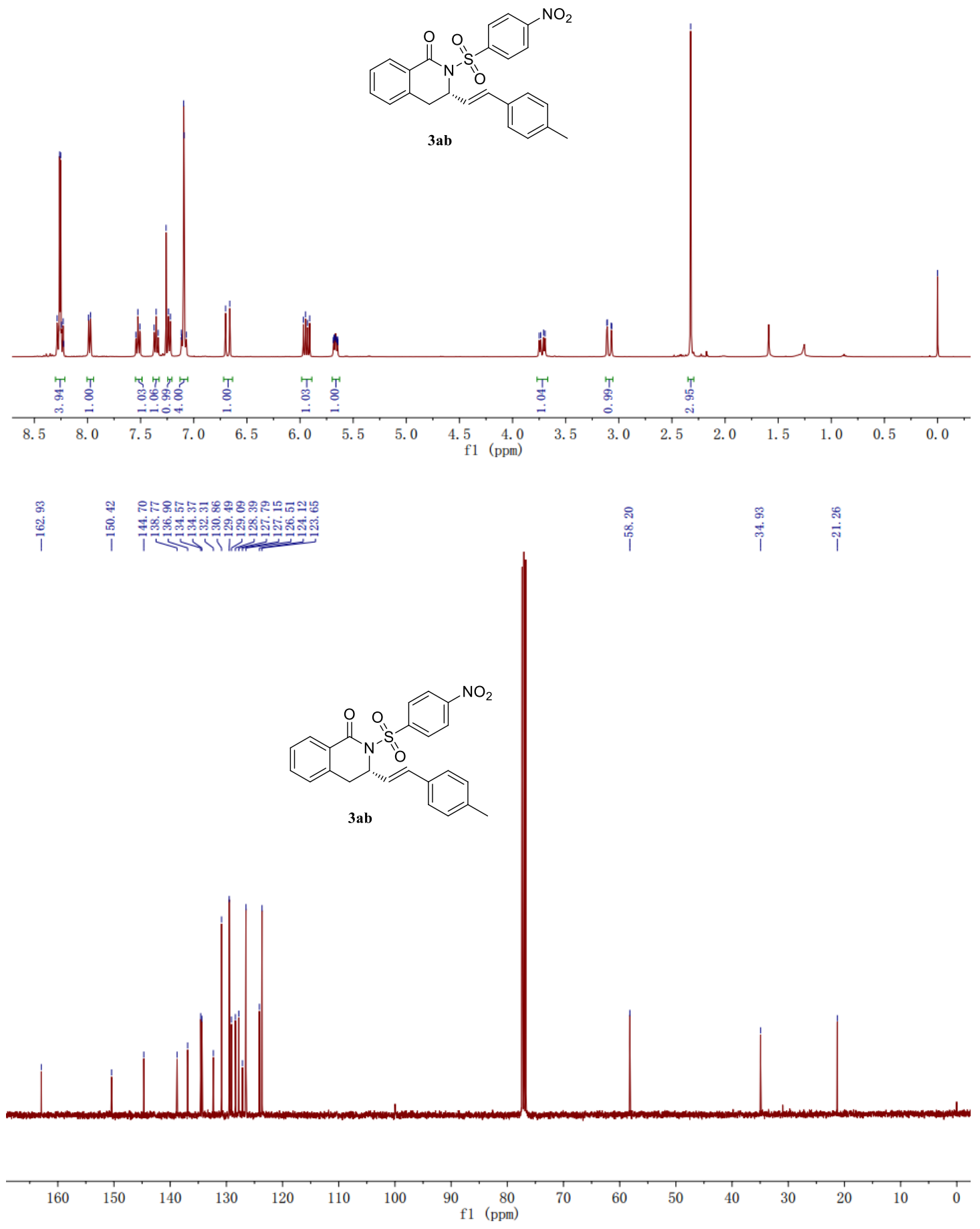

S27 


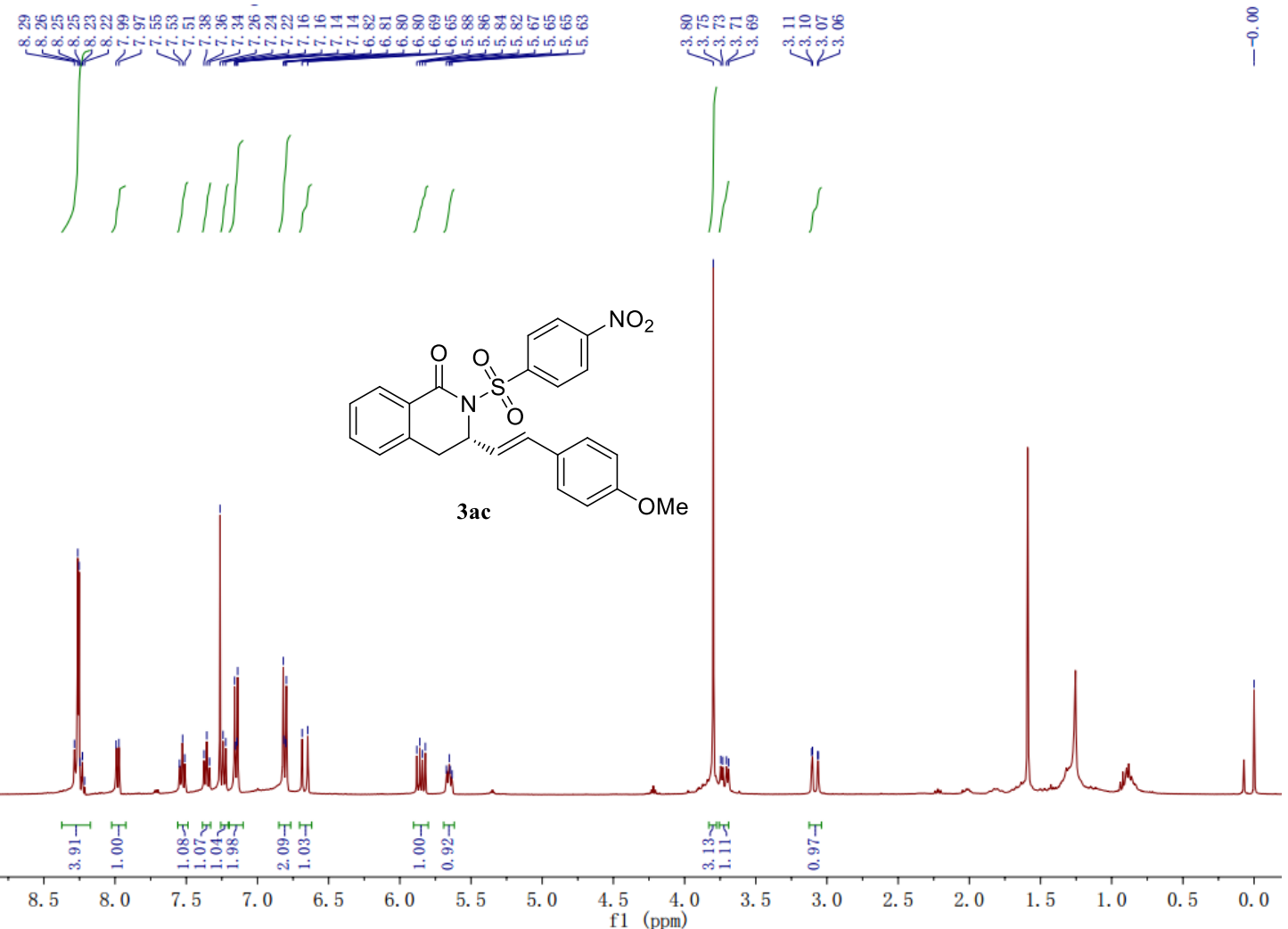

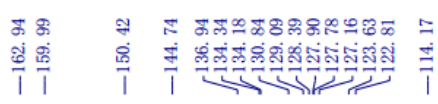
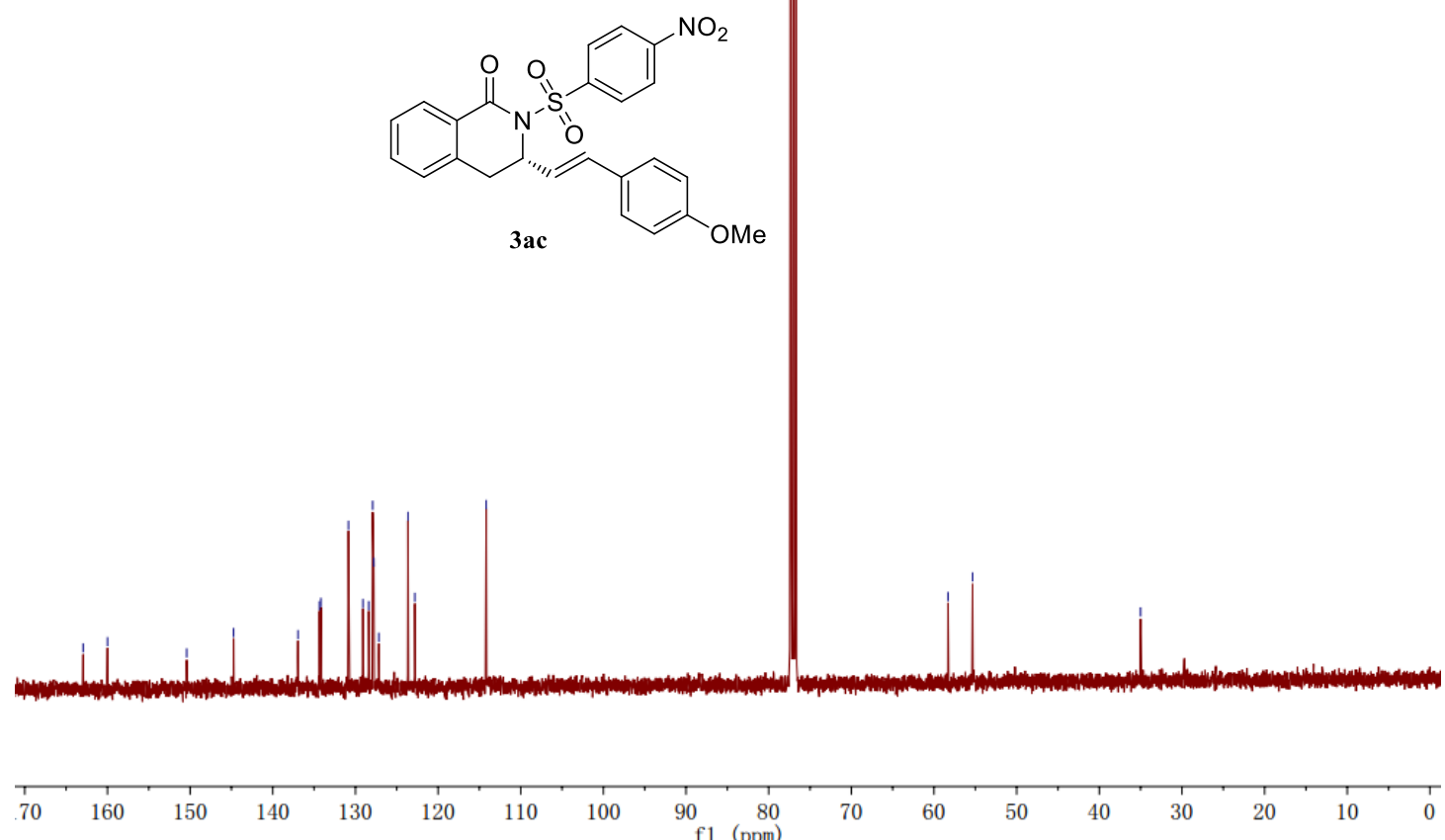


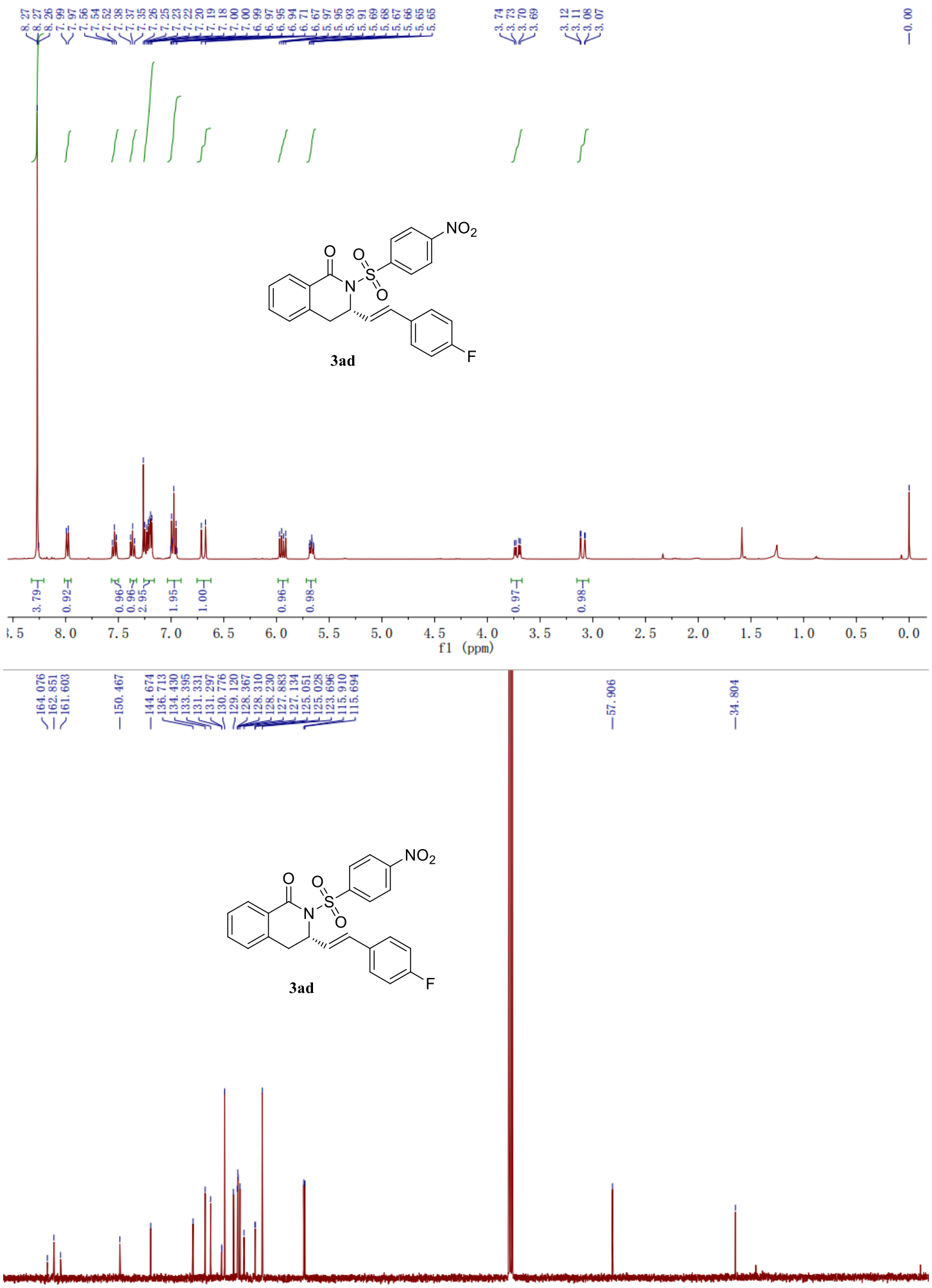

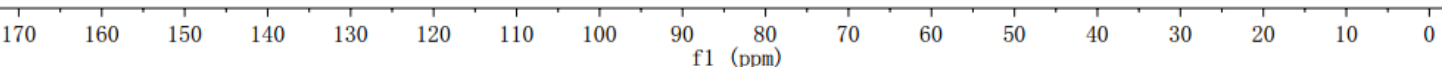


$3 a e$
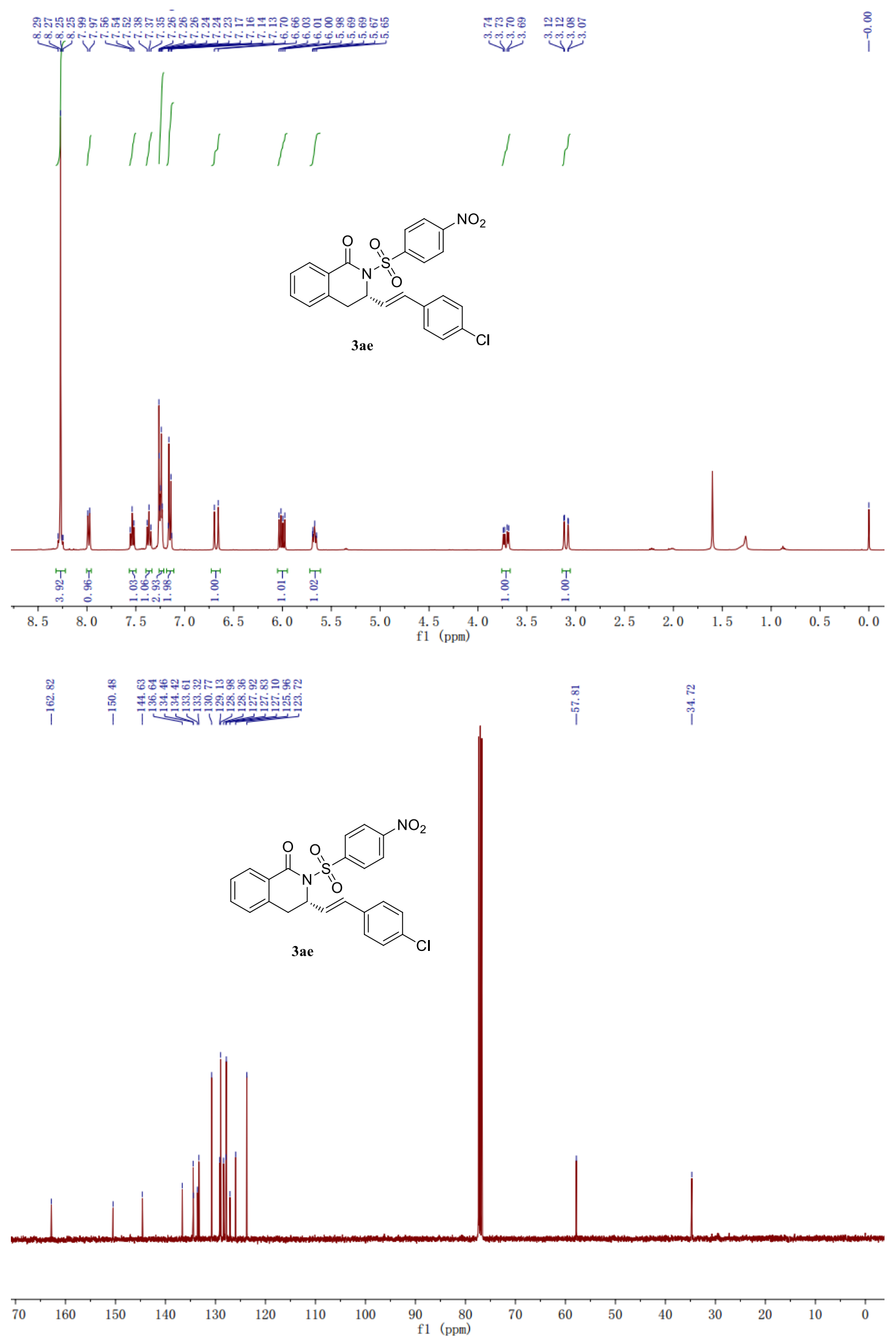

S30 


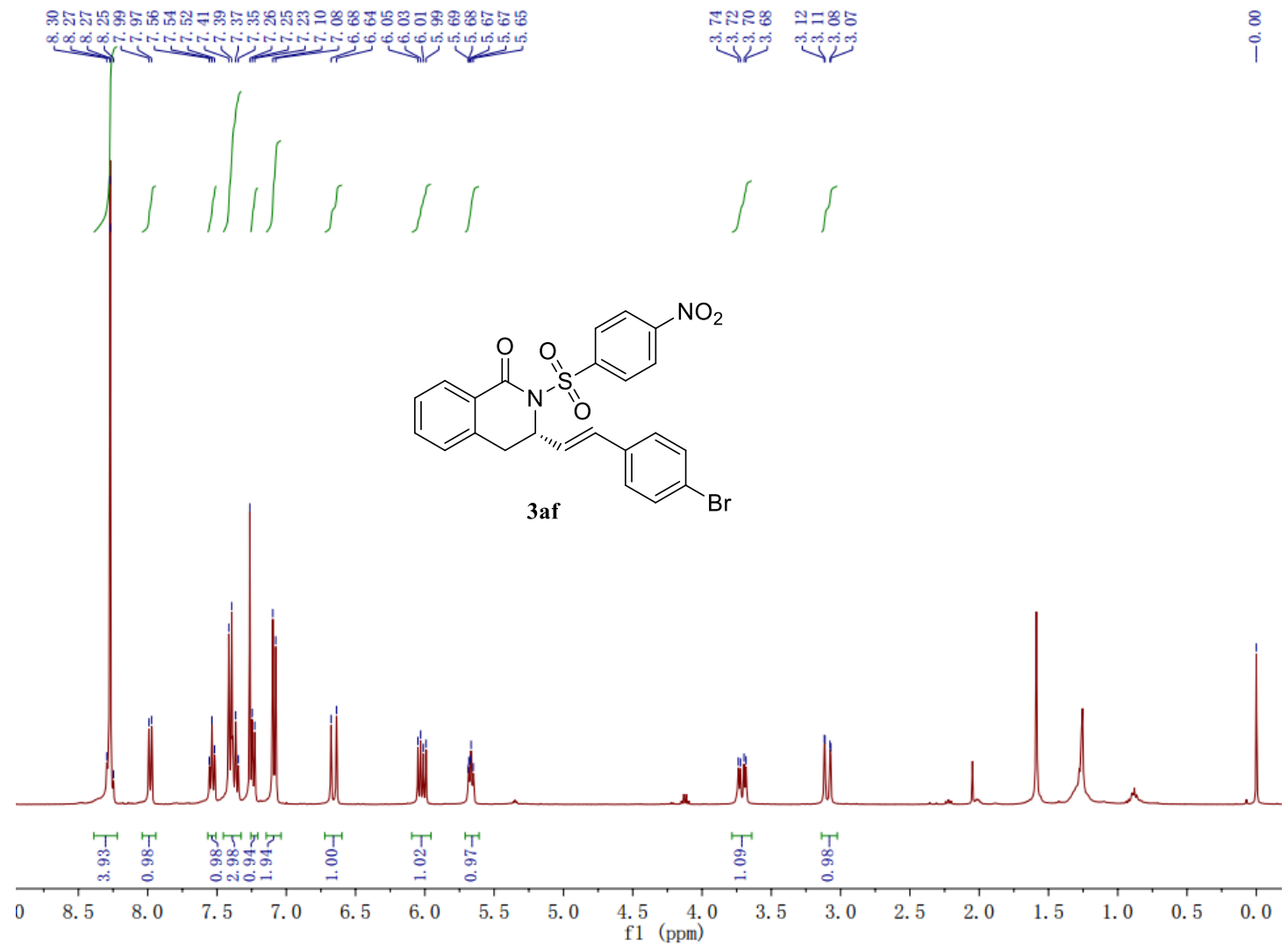

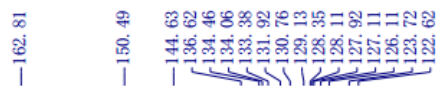
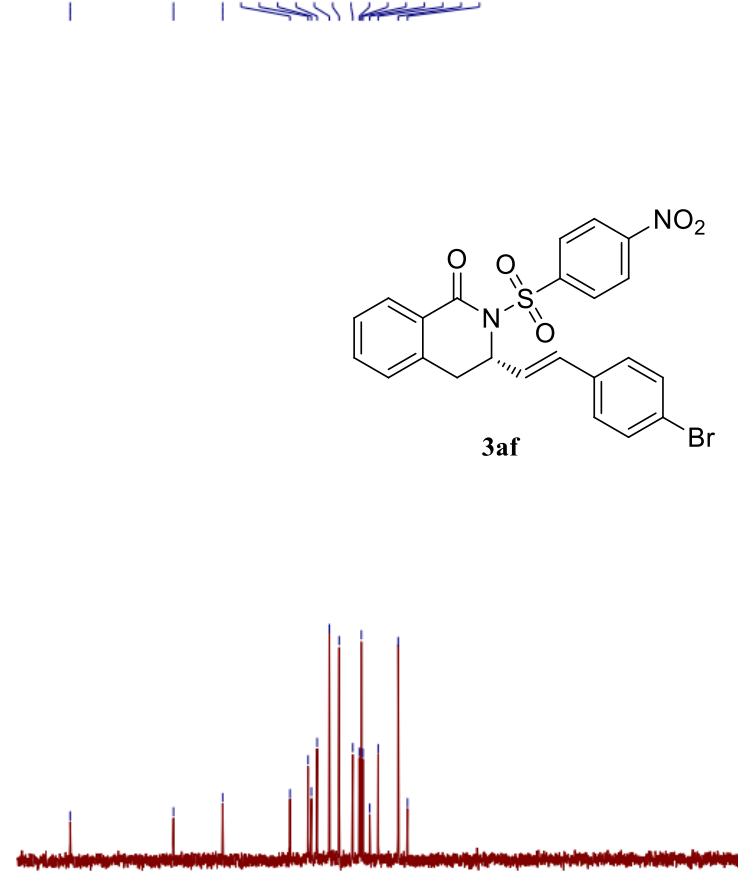

$\stackrel{\substack{1 \\ 0}}{\substack{0 \\ 0}}$

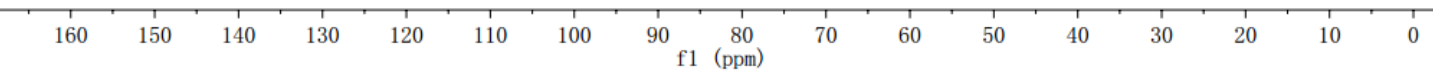


$3 a g$

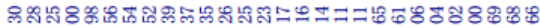

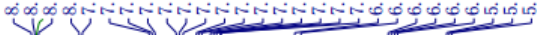

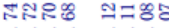

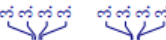
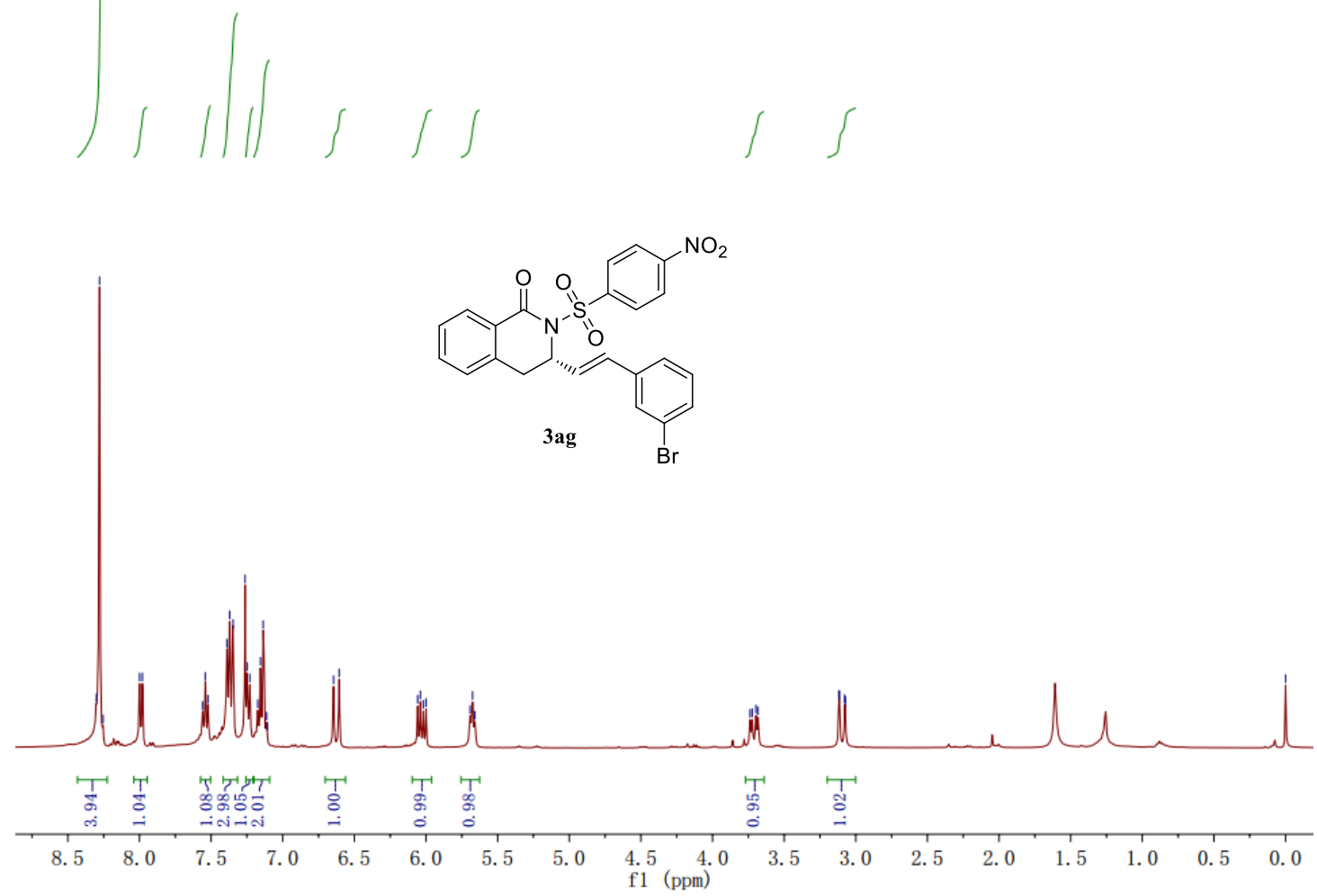

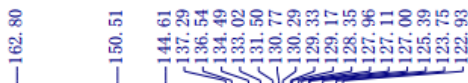
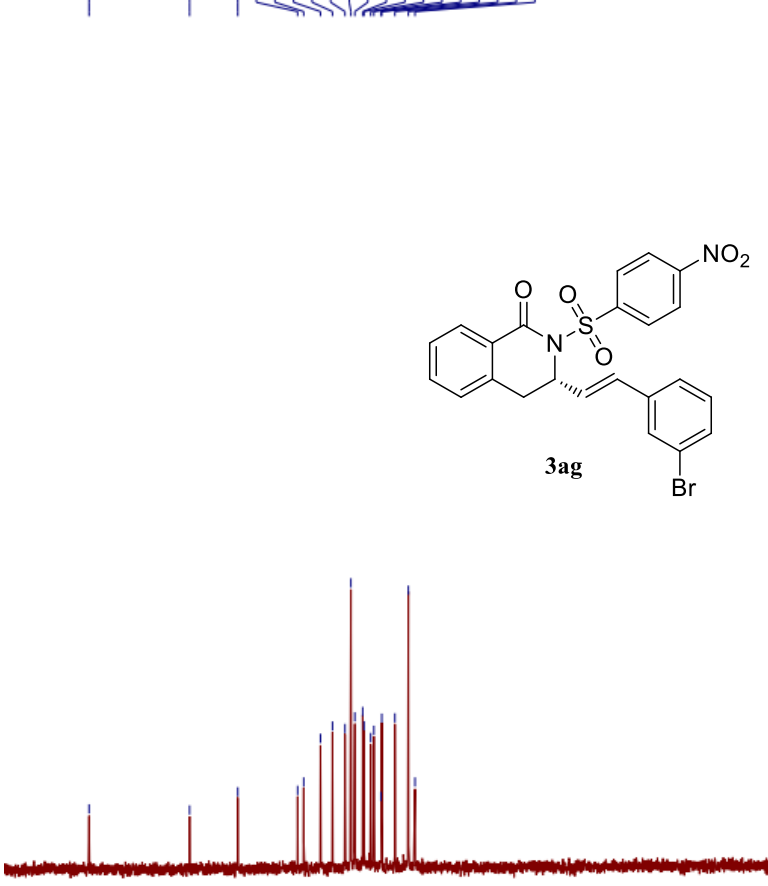

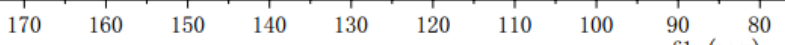

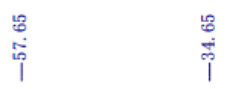

i 
3ah

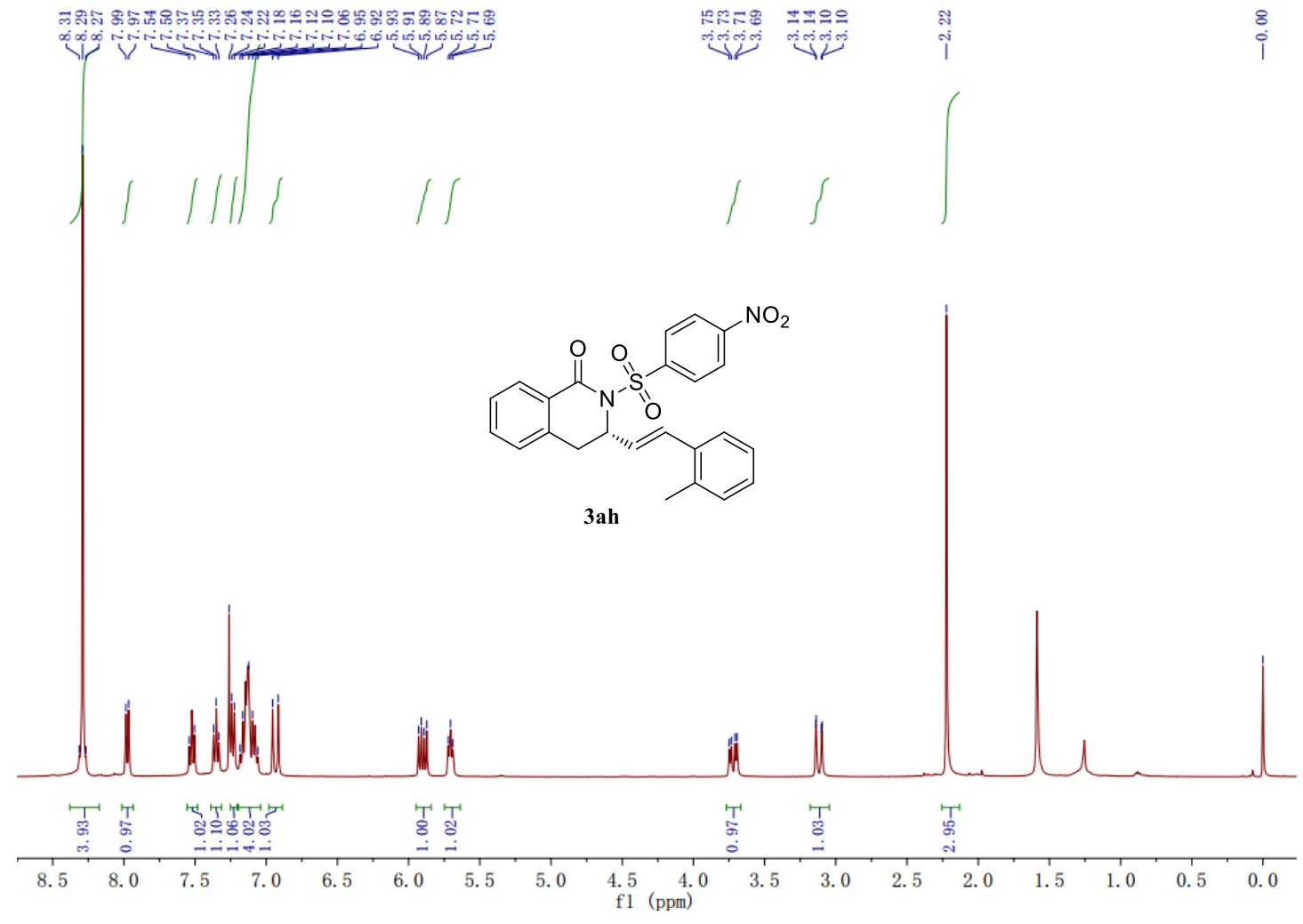

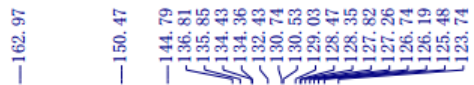

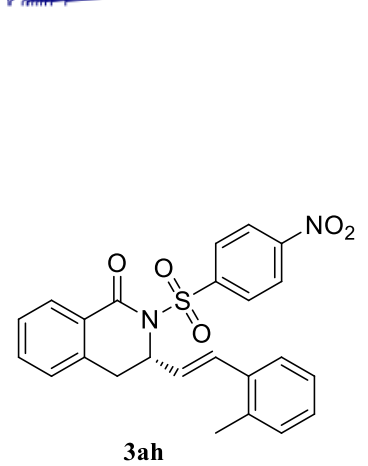

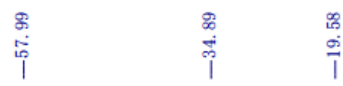

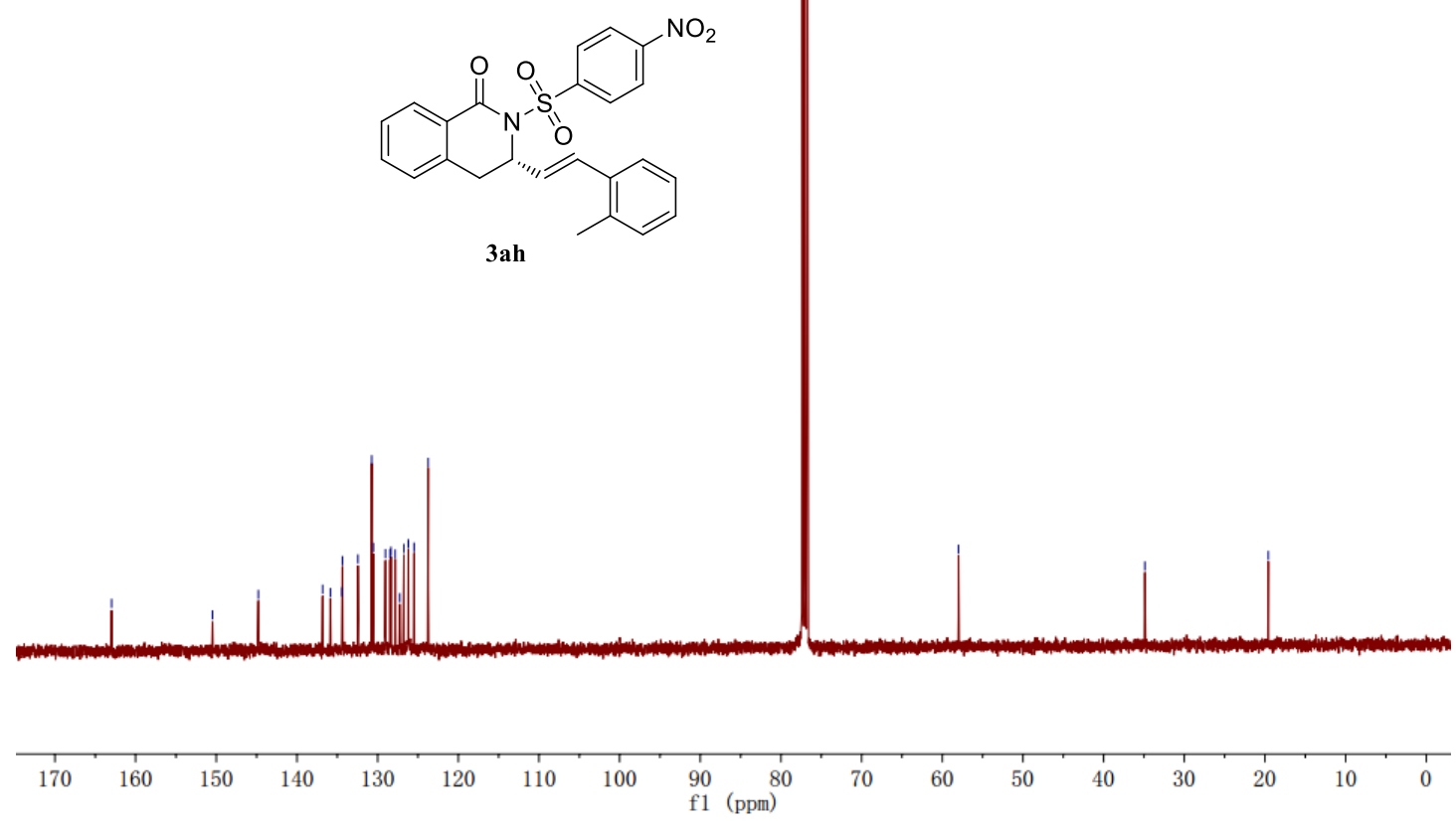


3ai

ஜ

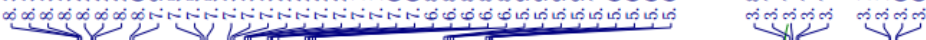

$\stackrel{8}{1}$

$\| 11 / 1$
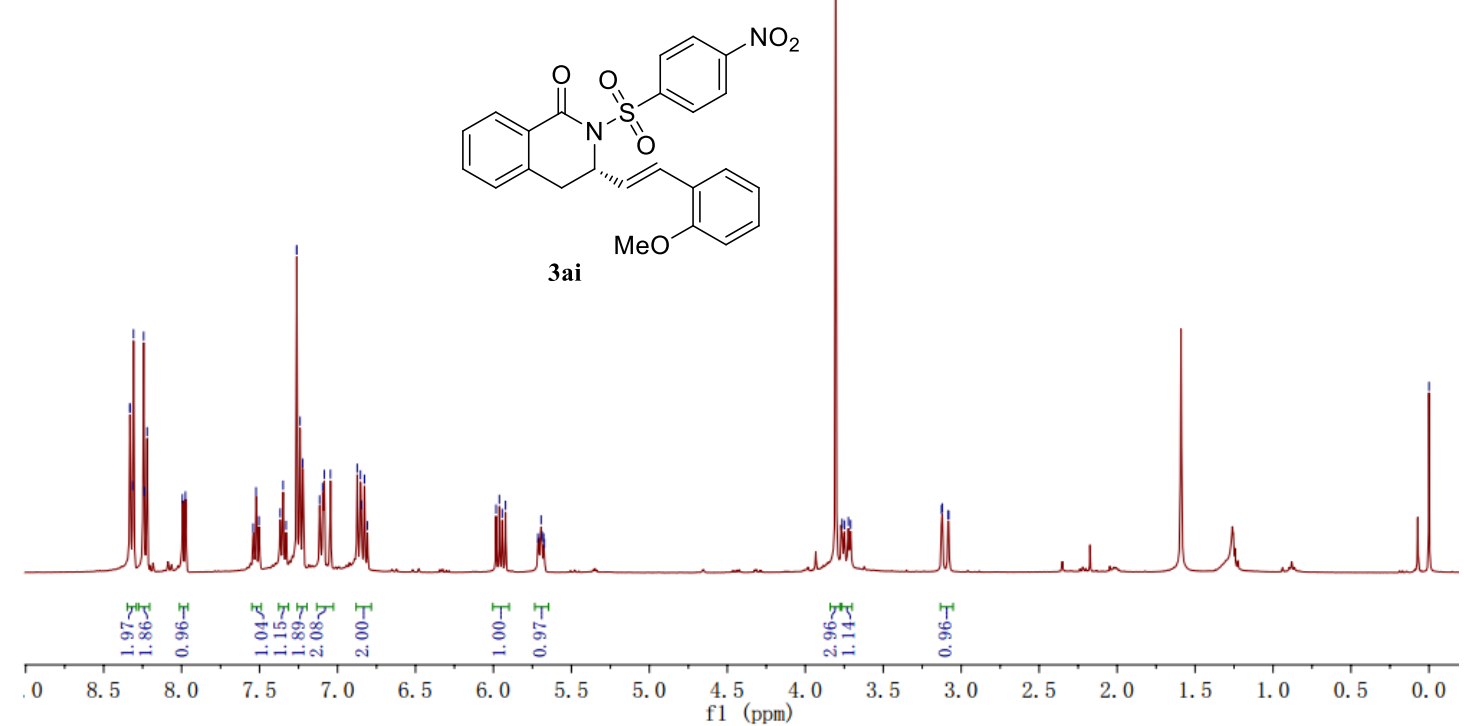

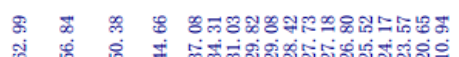

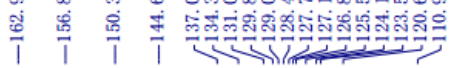

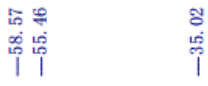

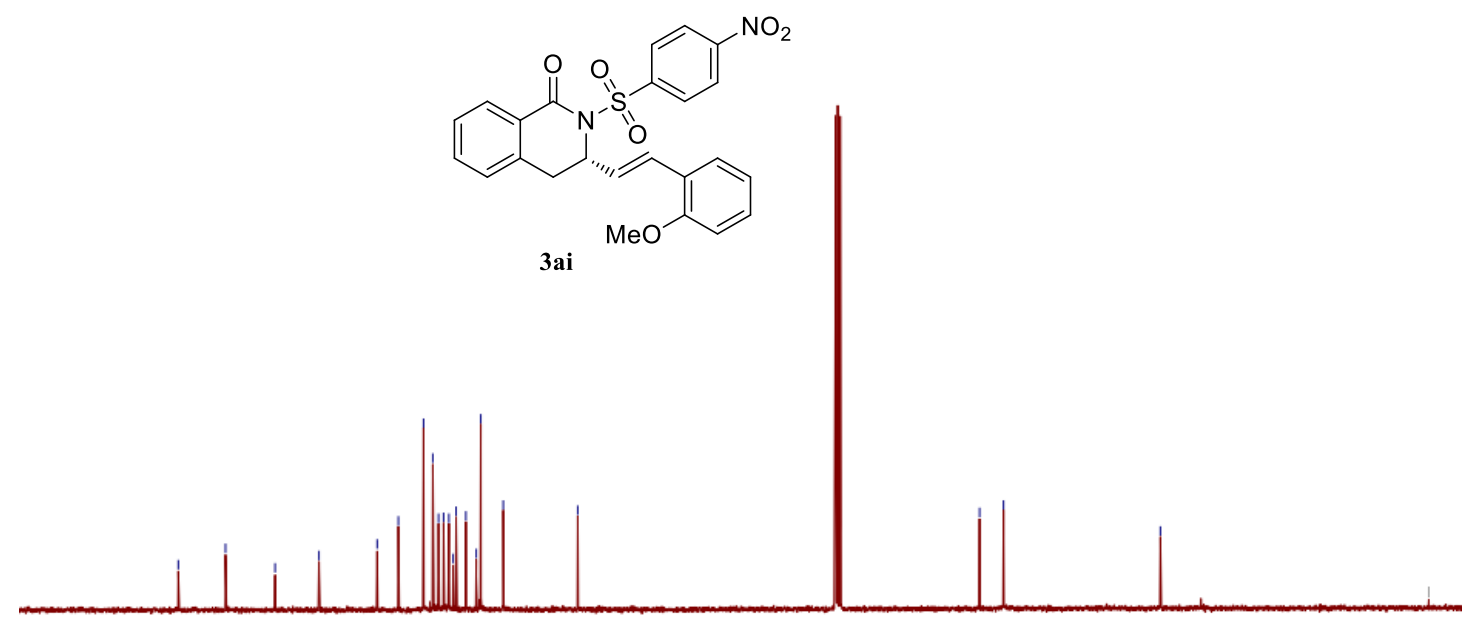

3ai

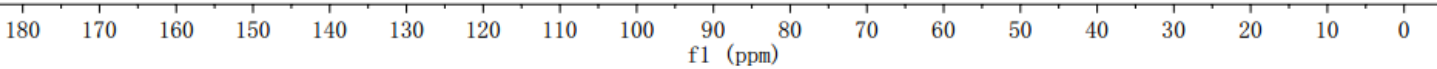


3aj

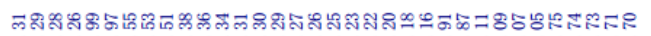

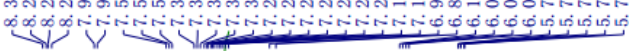

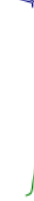

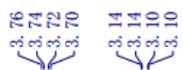

8
$i$
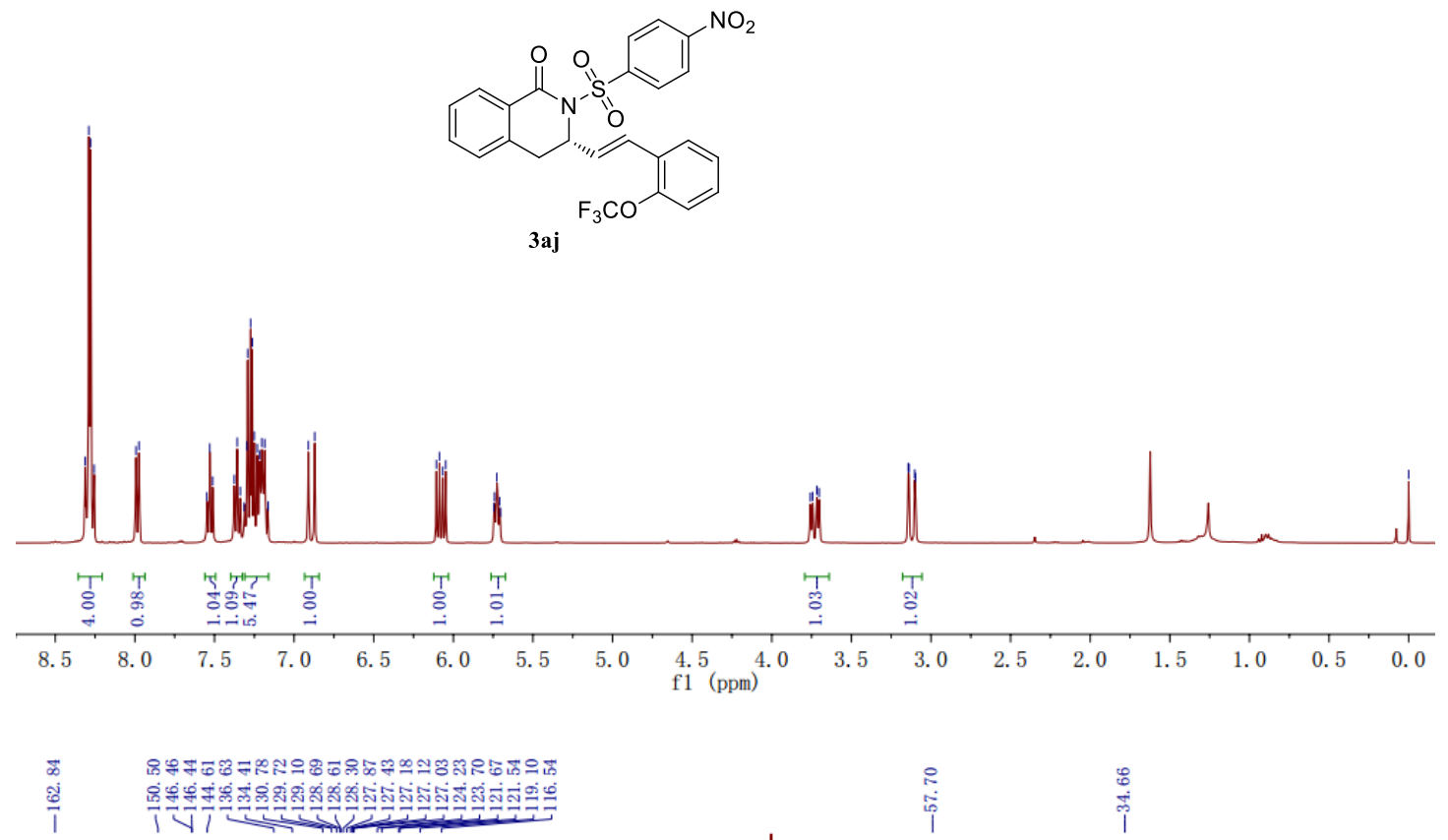

운

$\stackrel{\leftrightarrow}{i}$

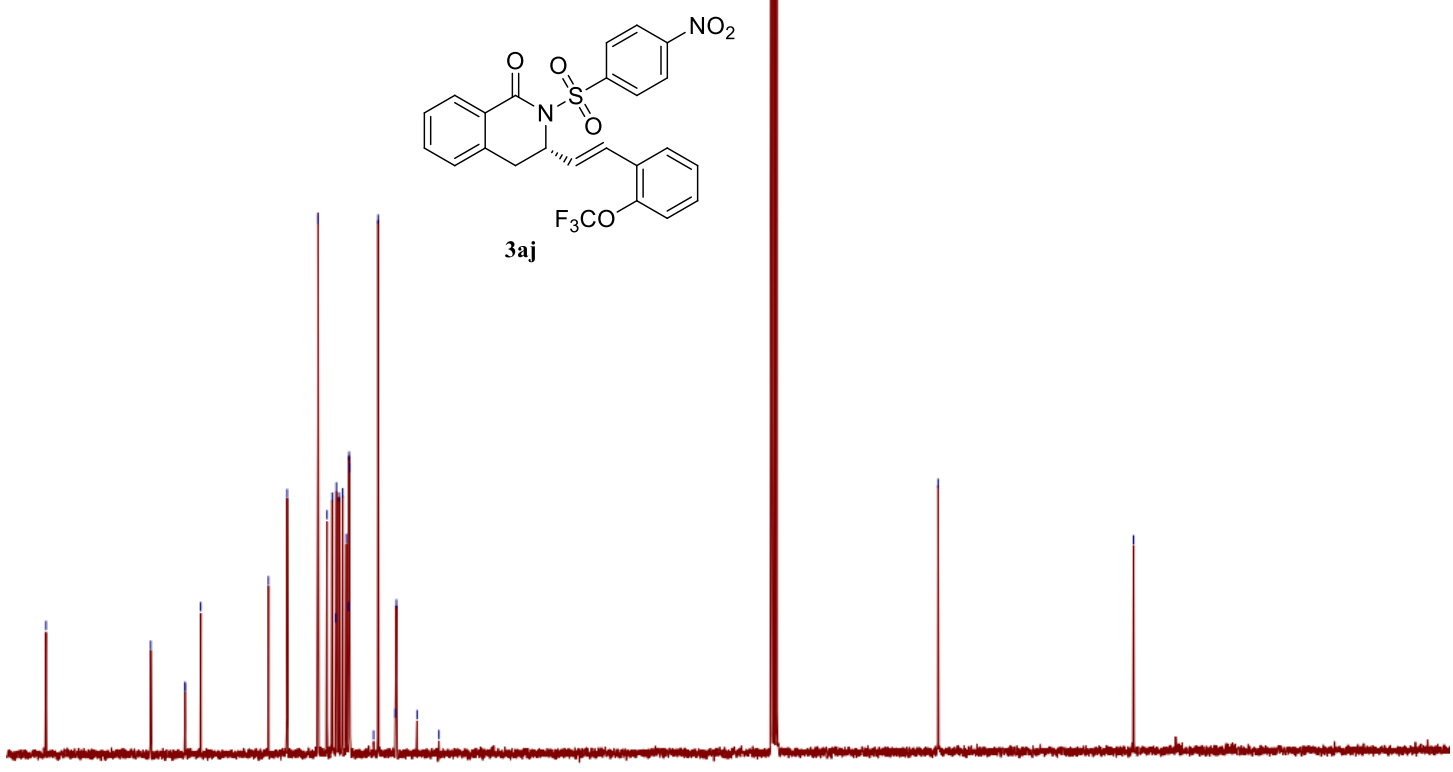

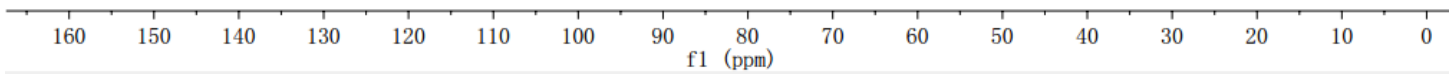

S35 
3ak

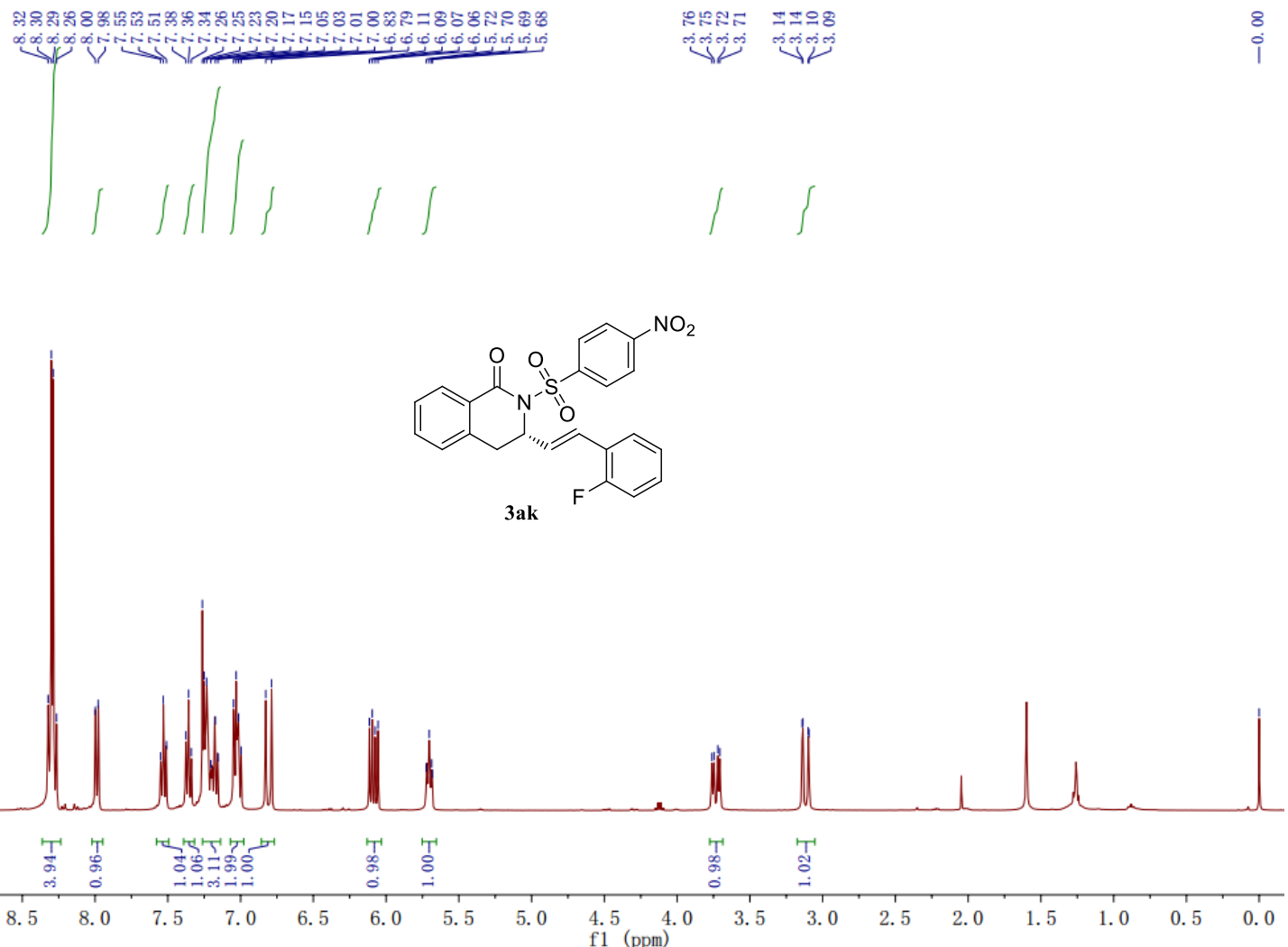

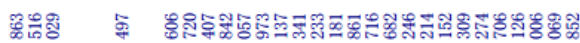

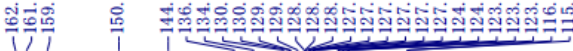
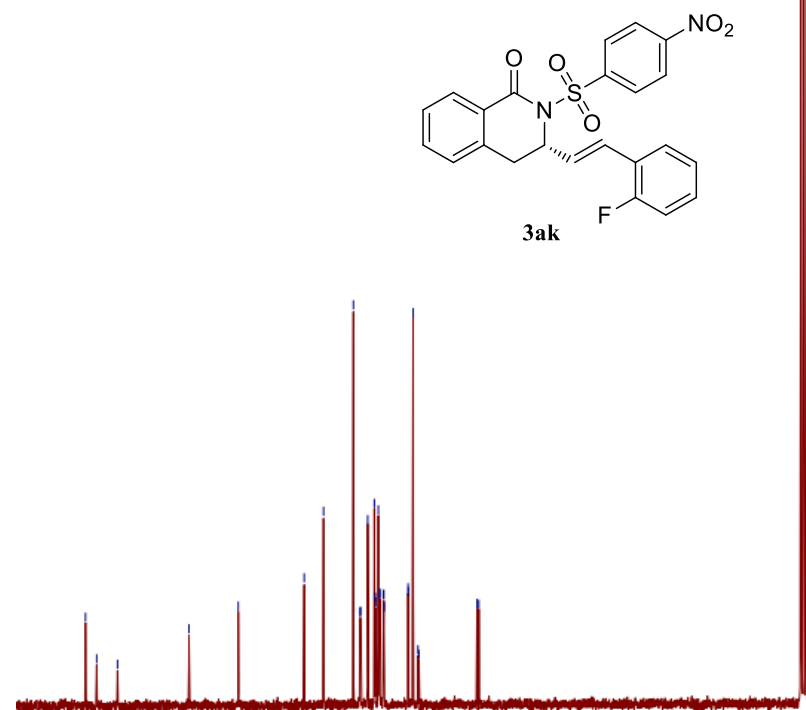

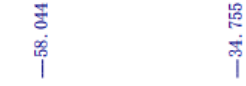

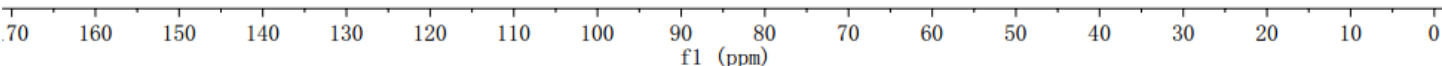


๓ூ

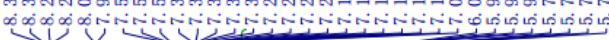

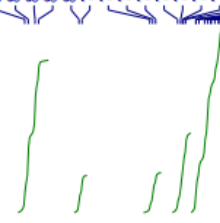<smiles>C=C=CC1Cc2ccccc2C(=O)N1S(=O)(=O)c1ccc([N+](=O)[O-])cc1</smiles>

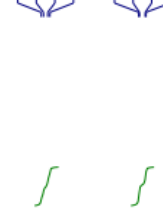 \\ i} (1) 
$3 \mathbf{a m}$
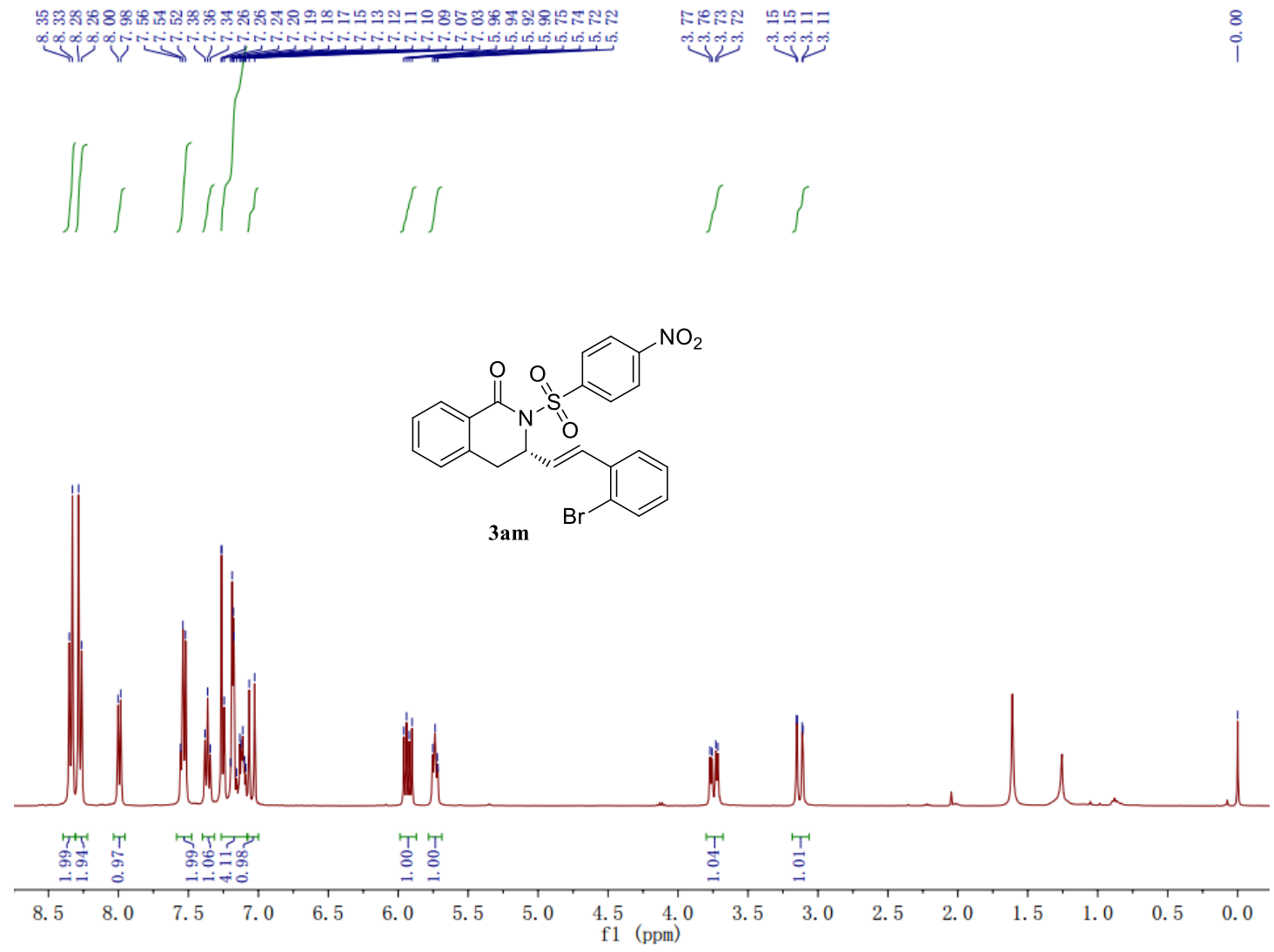

D.

管

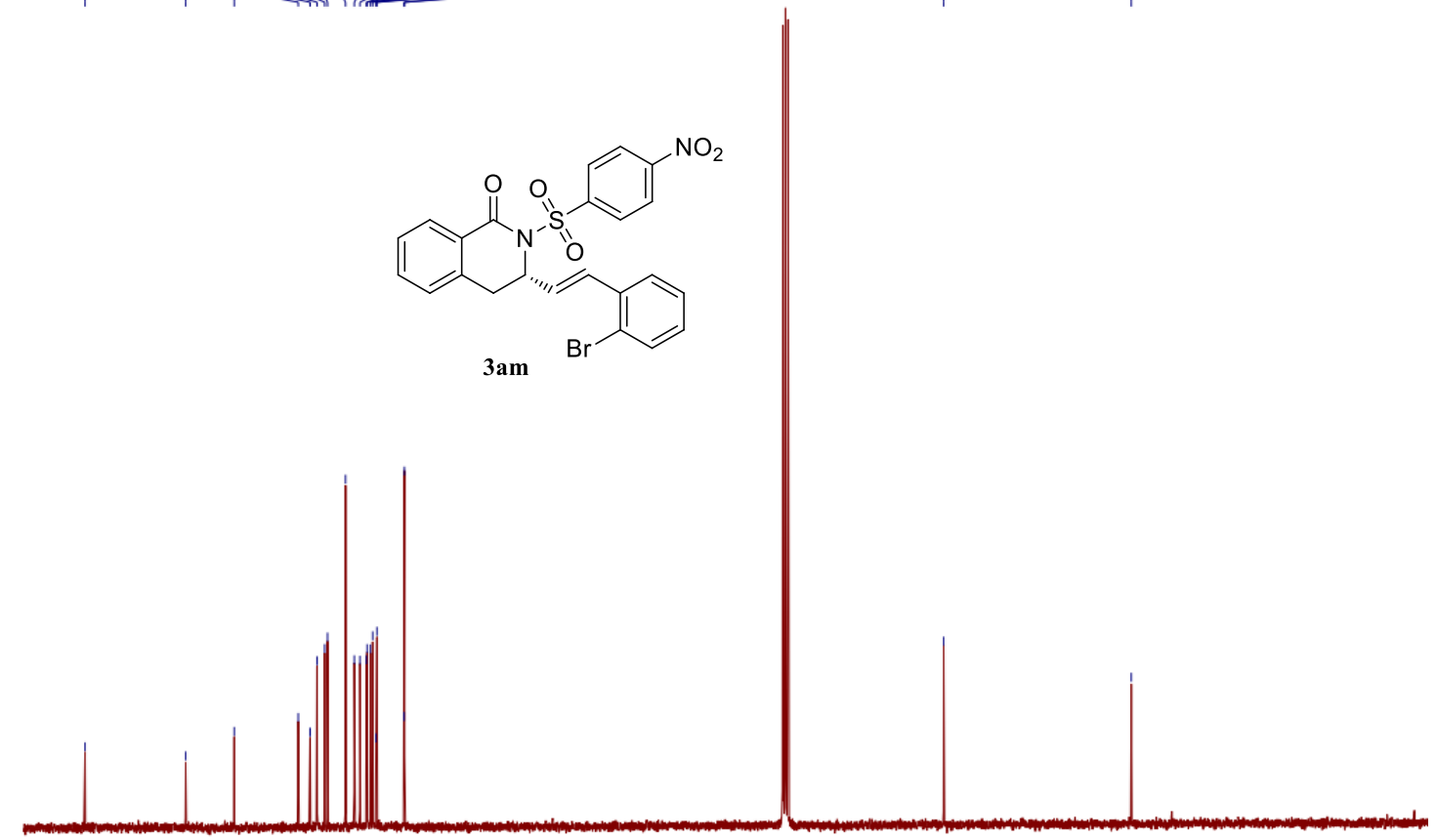

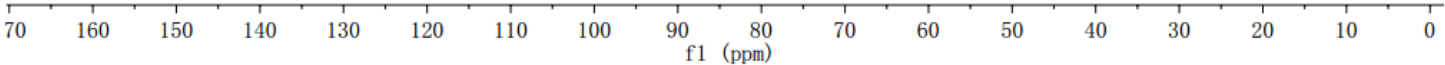


3 an

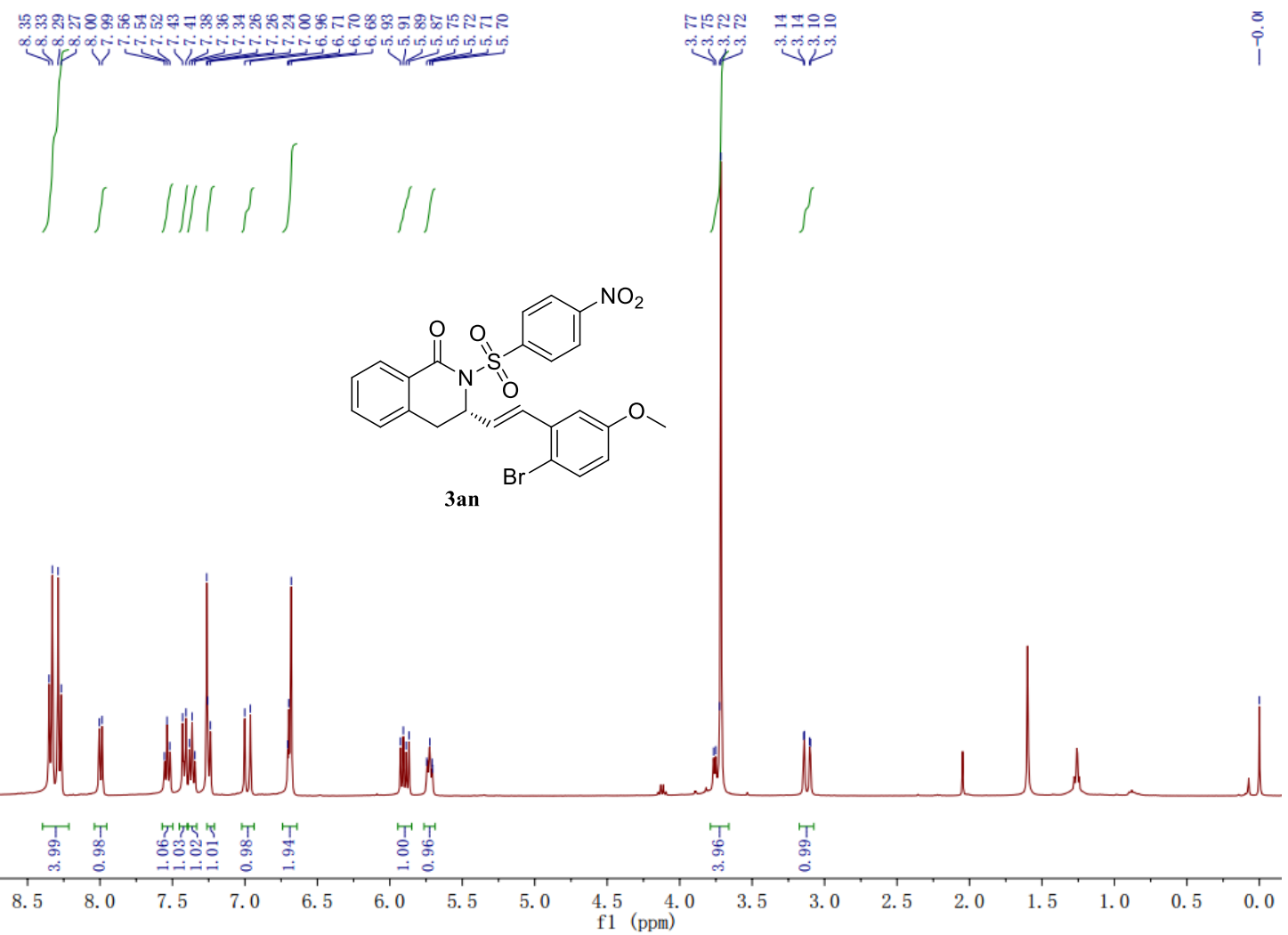

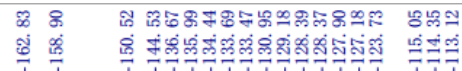

।
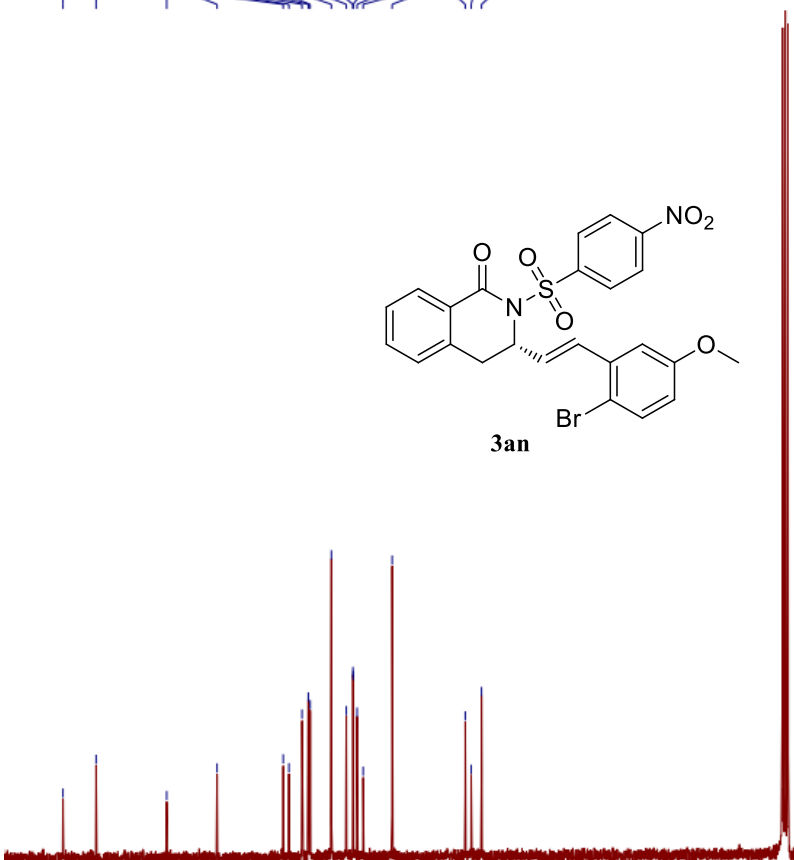

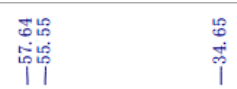

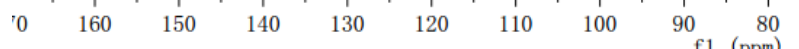




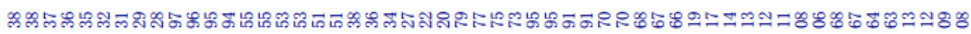
年
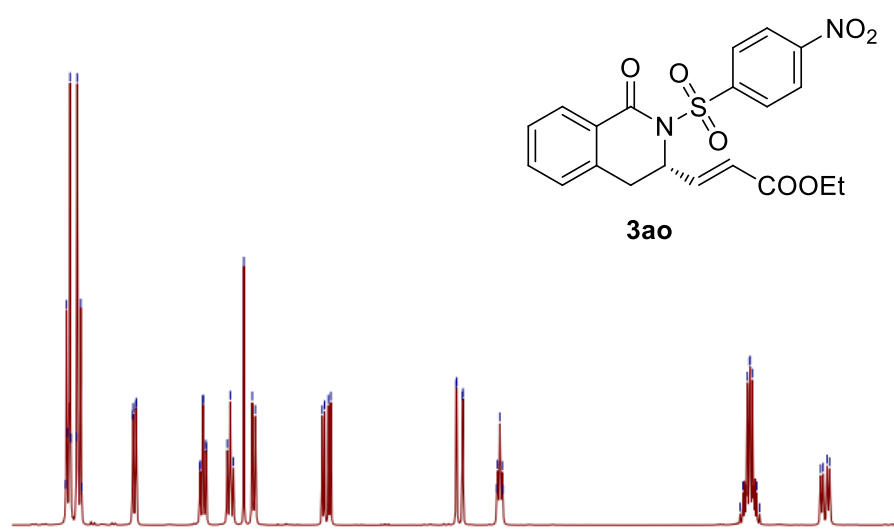

$3 a o$

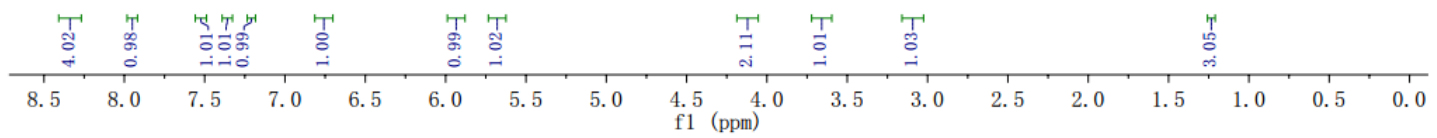

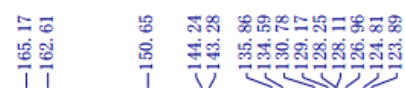

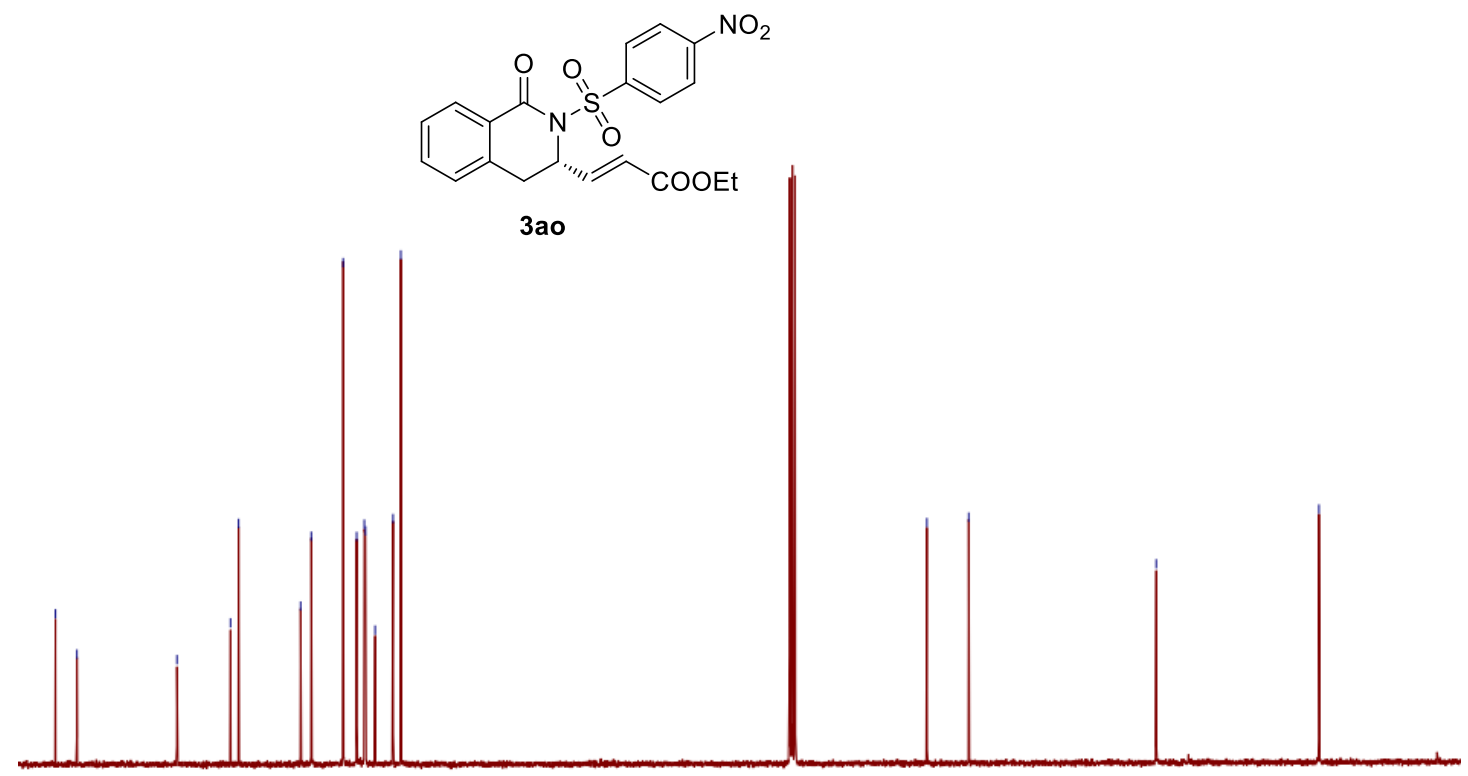

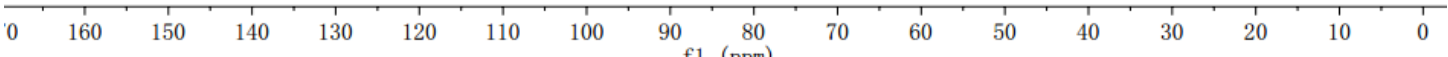


3 ap

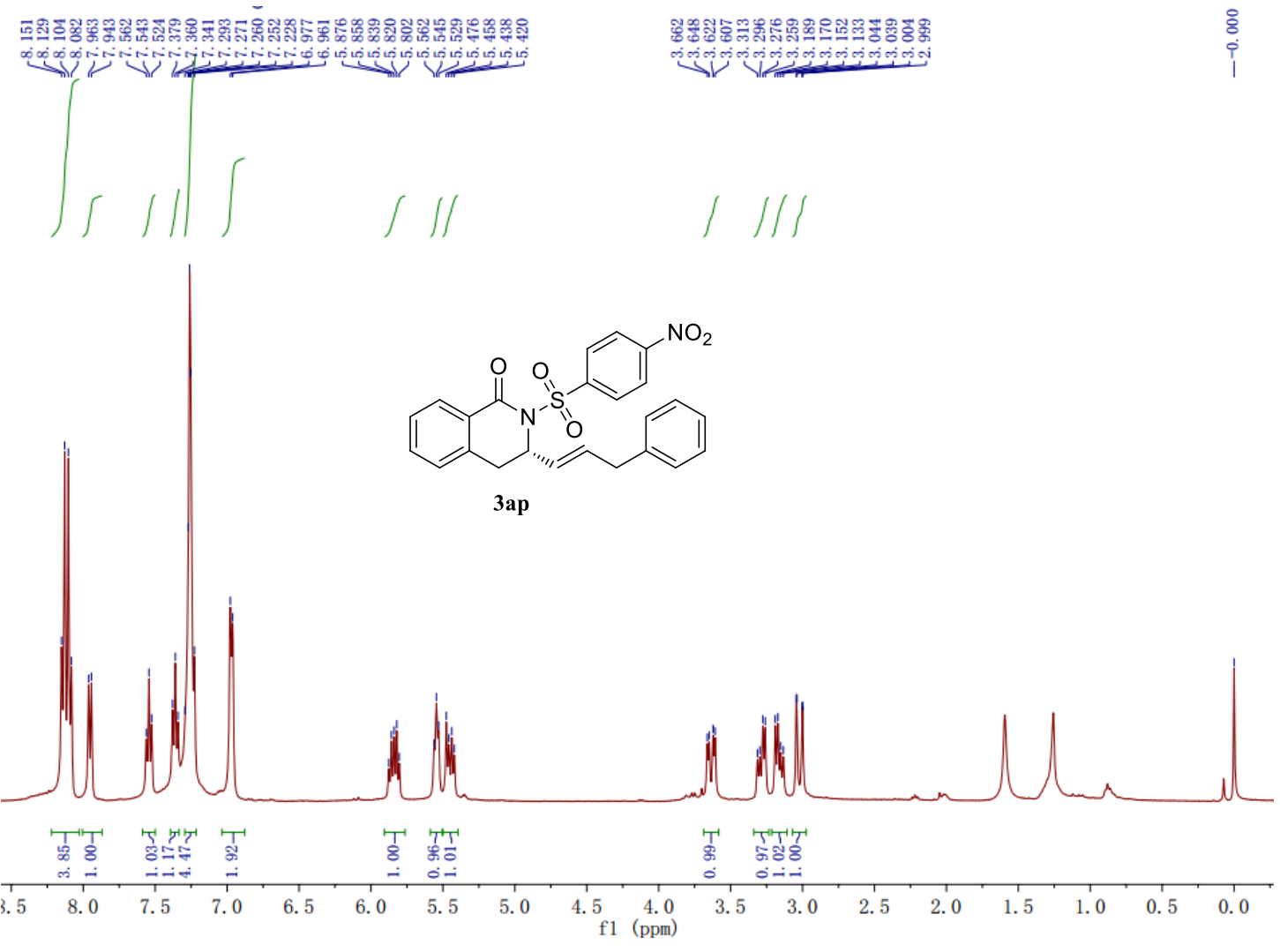

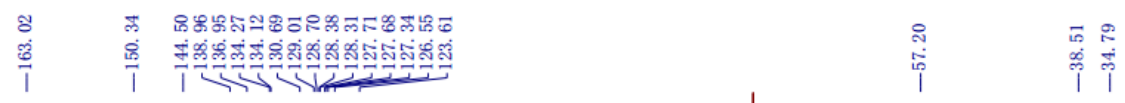

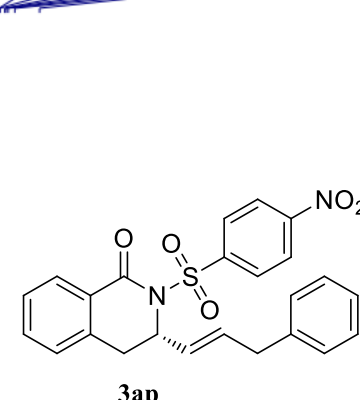

3 ap
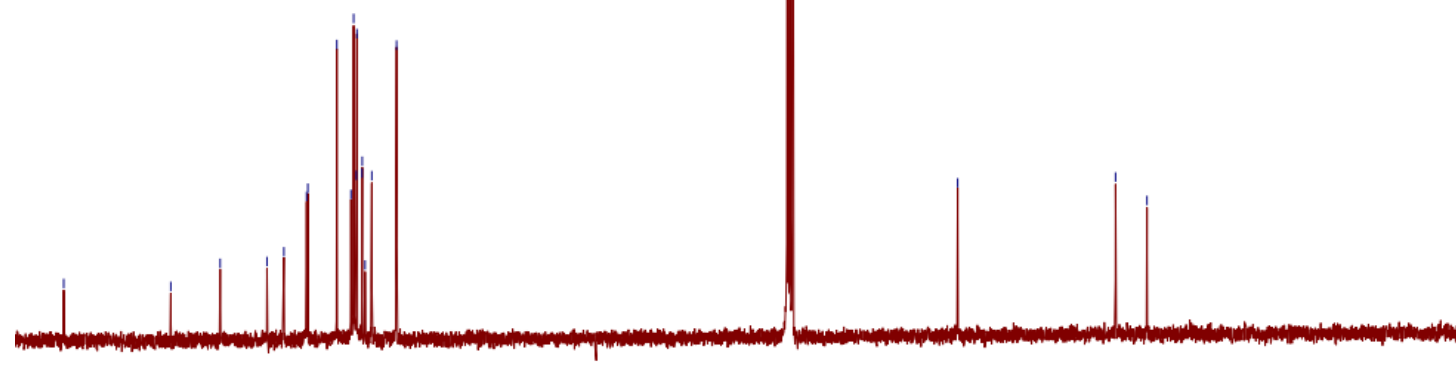

$\begin{array}{llllllll}160 & 150 & 140 & 130 & 120 & 110 & 100 & 90\end{array}$

f1 (ppm) 
$3 \mathbf{a q}$

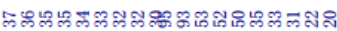

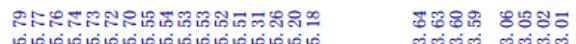

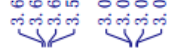

8

$\underbrace{\infty}_{0,0000000}$

(1)

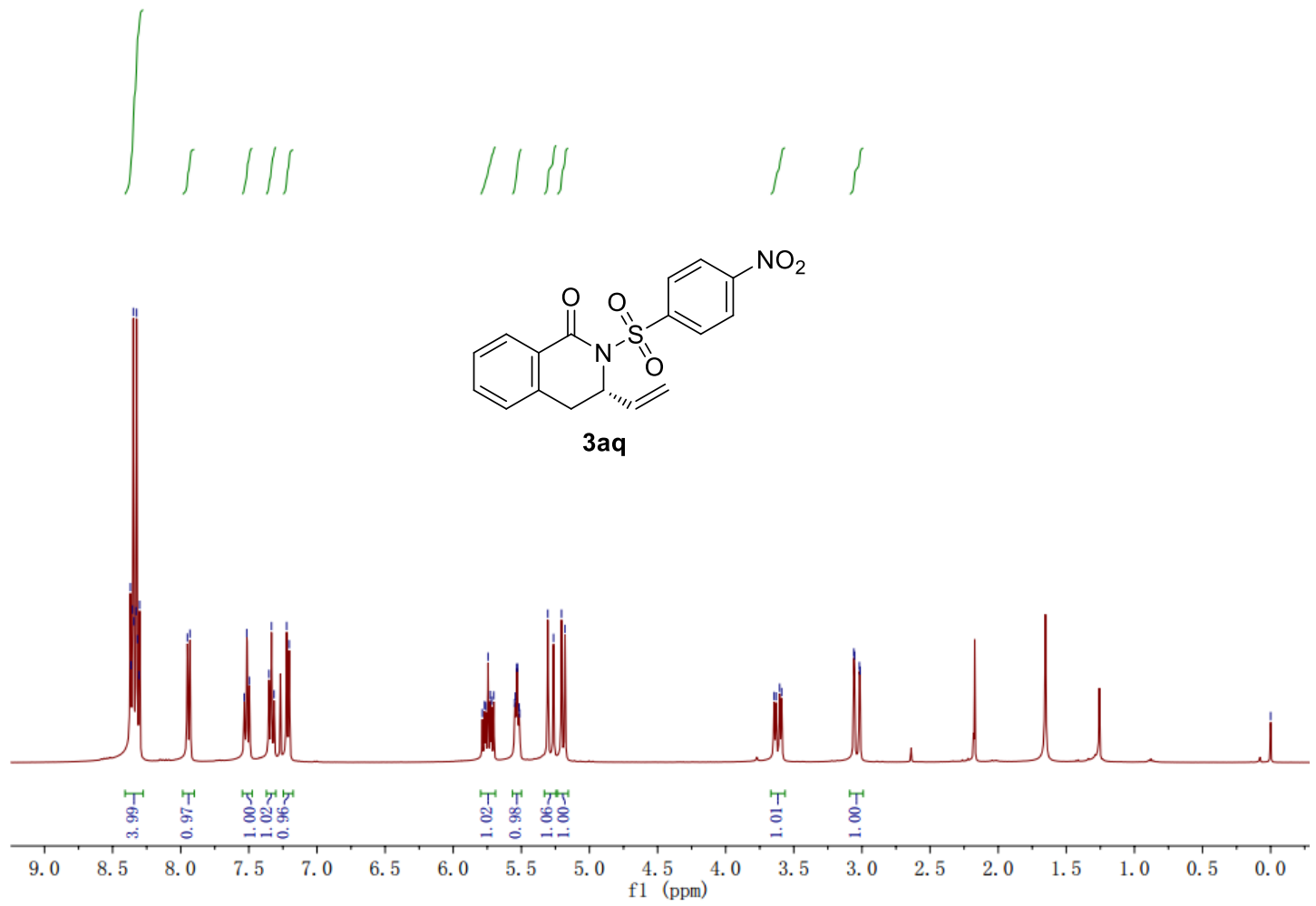

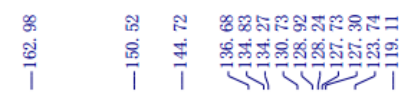

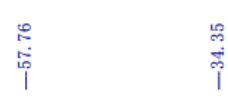<smiles>C=C[C@@H]1Cc2ccccc2C(=O)N1S(=O)(=O)c1ccc([N+](=O)[O-])cc1</smiles>

$3 a q$

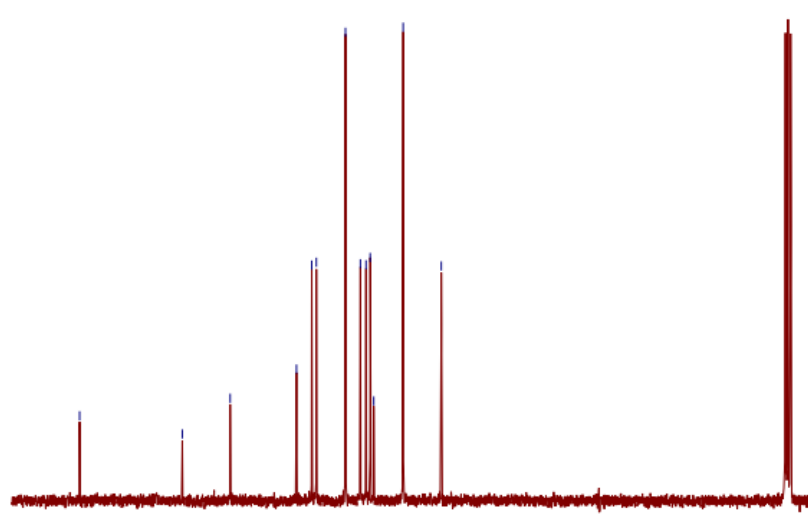

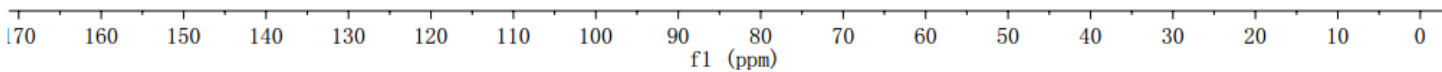


3pa
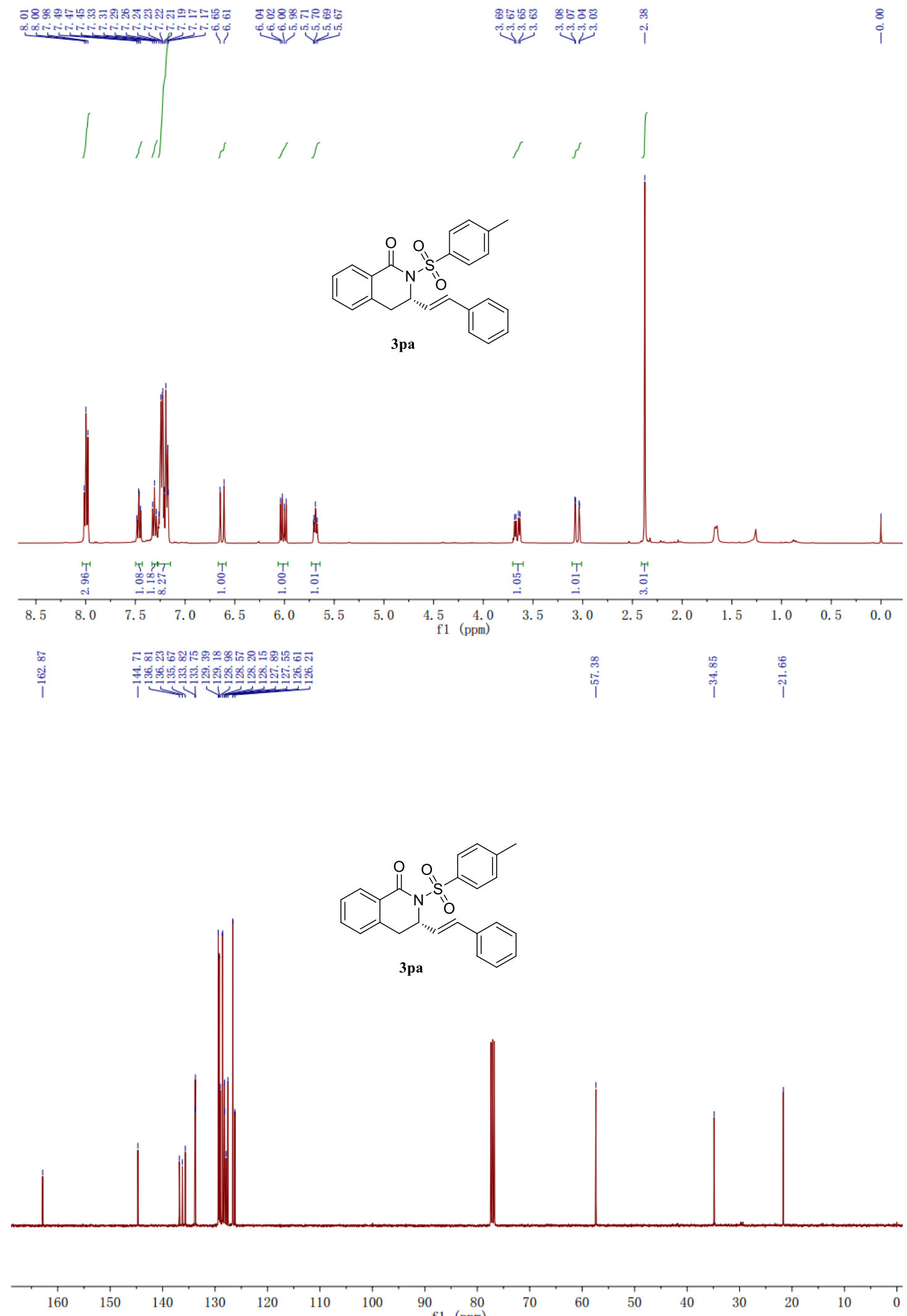
$3 q a$
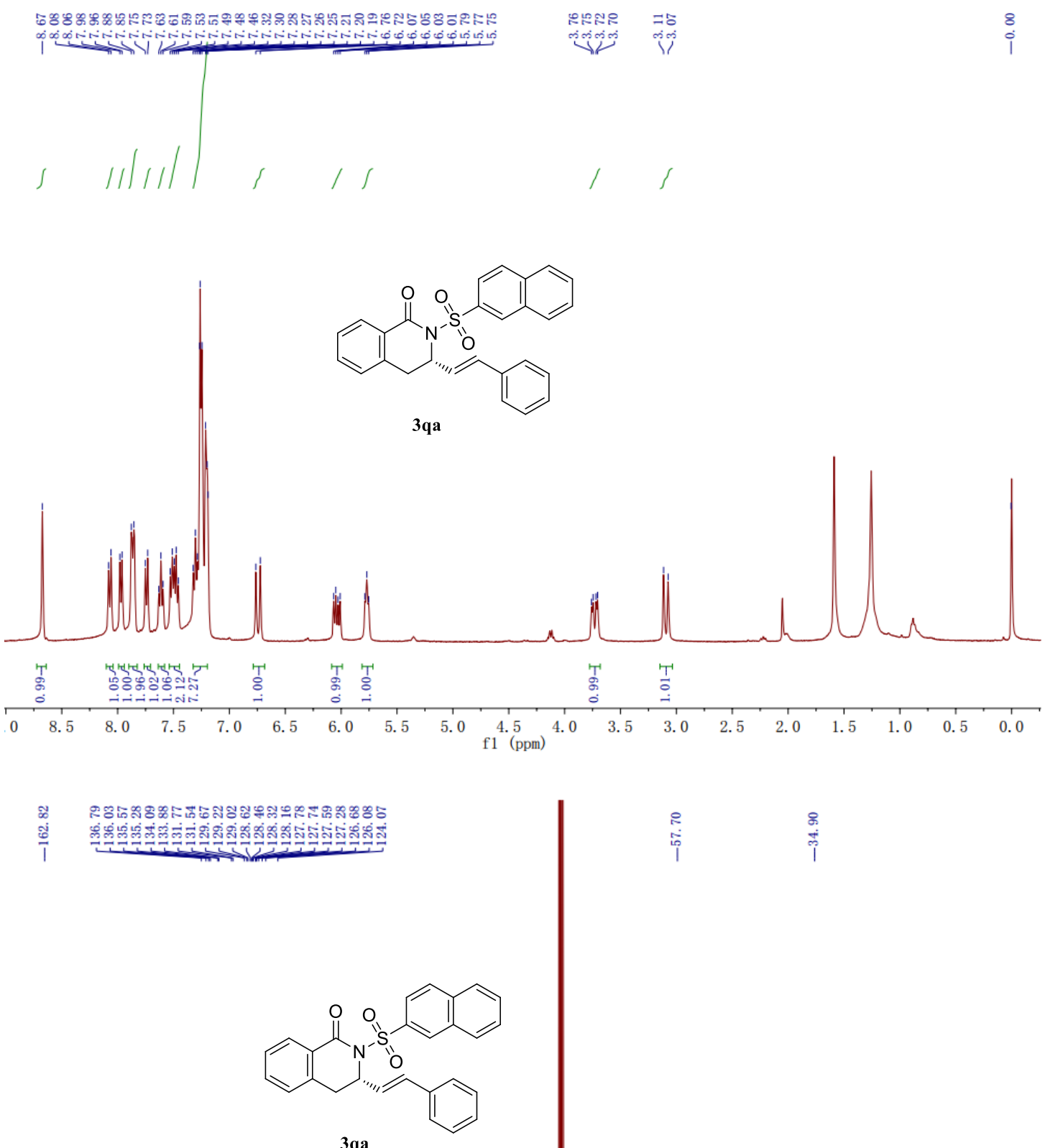

3qa

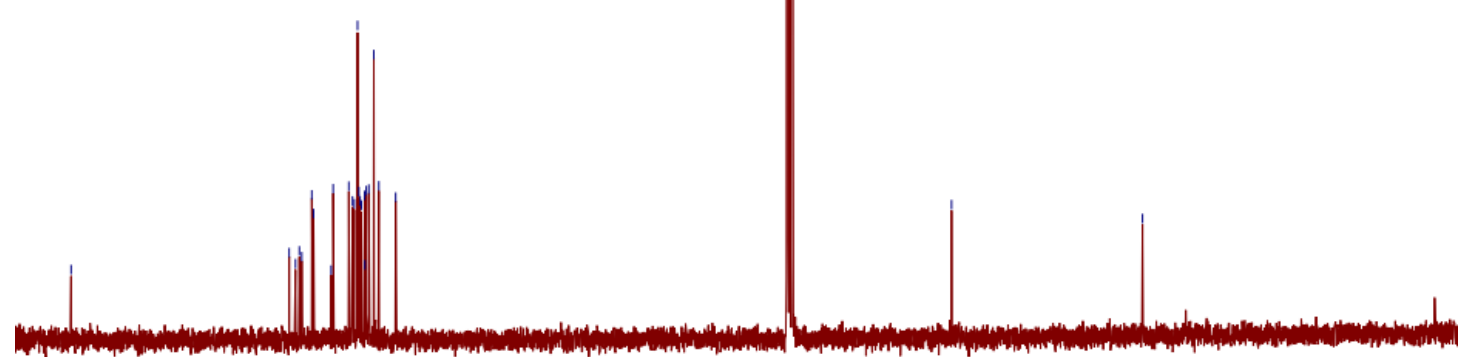

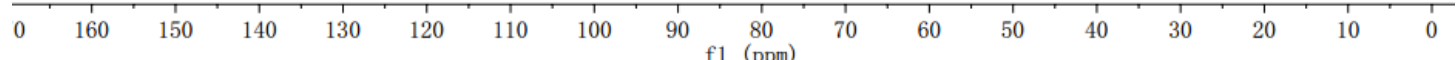


3ra
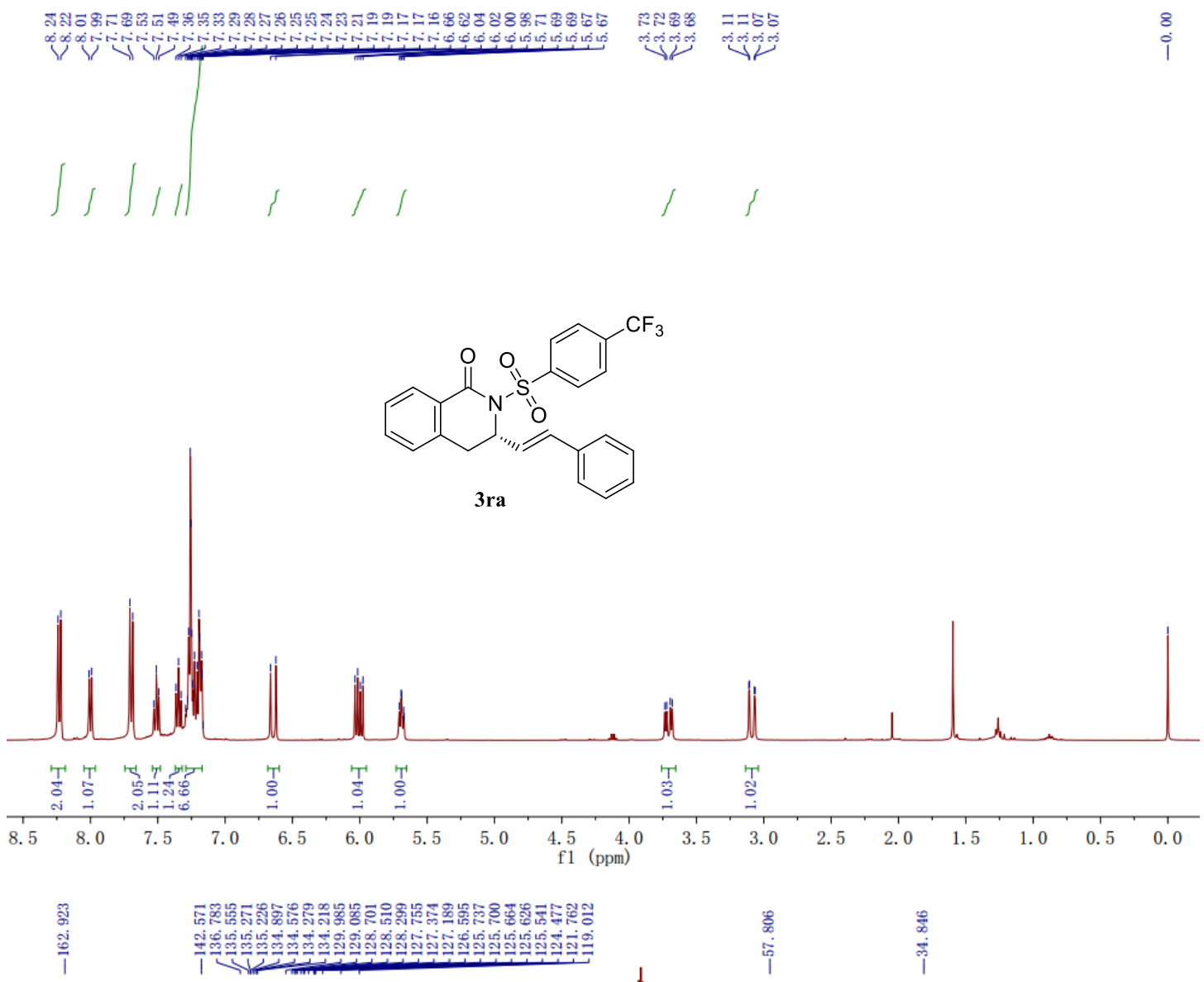

$\begin{array}{ll}0 & 0 \\ 0 & 0 \\ 0 & 0 \\ 0 & 0\end{array}$
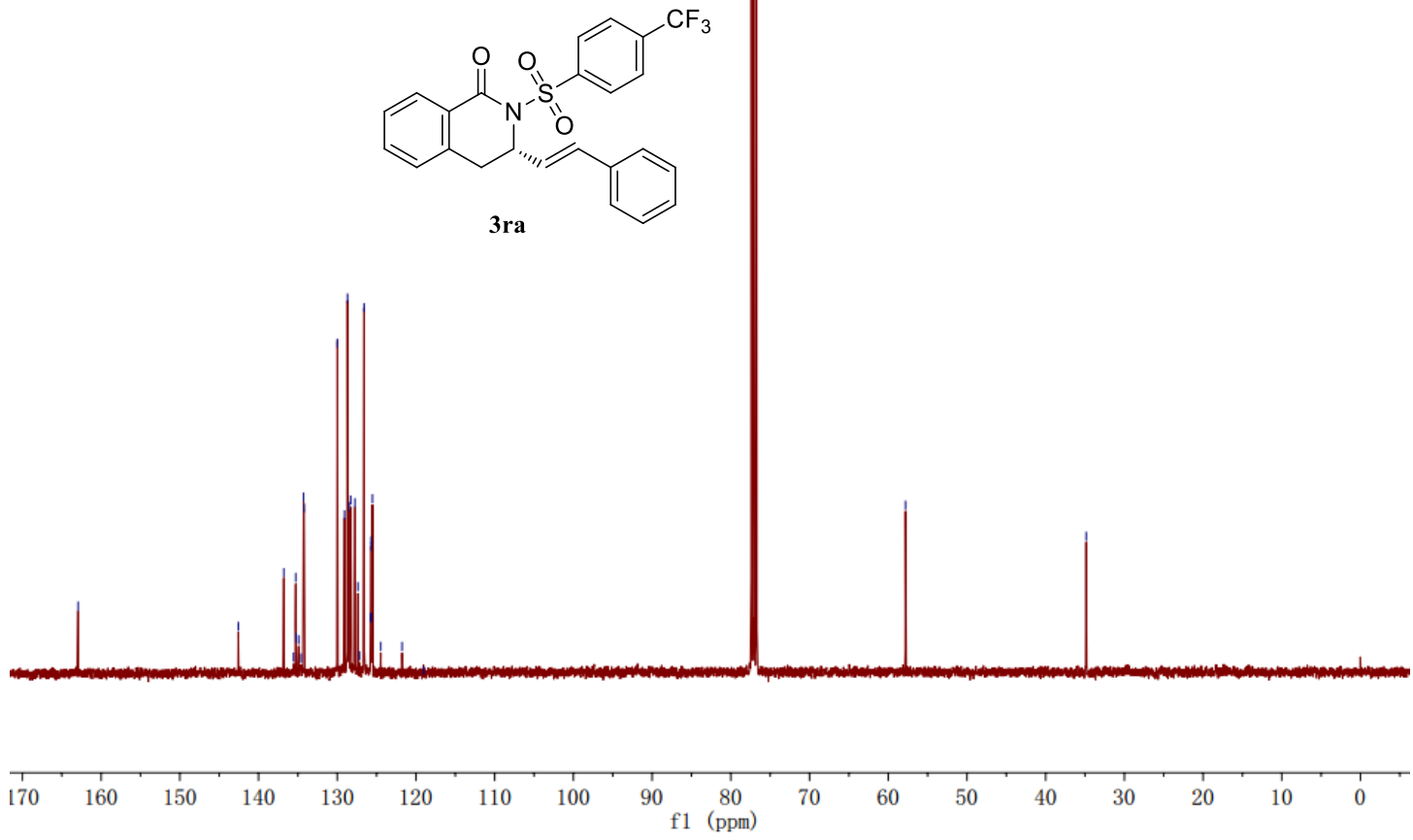

S45 
3sa

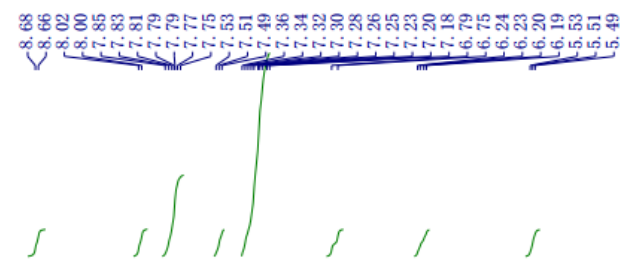

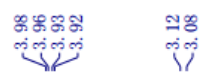

\begin{tabular}{l}
$\square$ \\
$\vdots$ \\
\hline
\end{tabular}
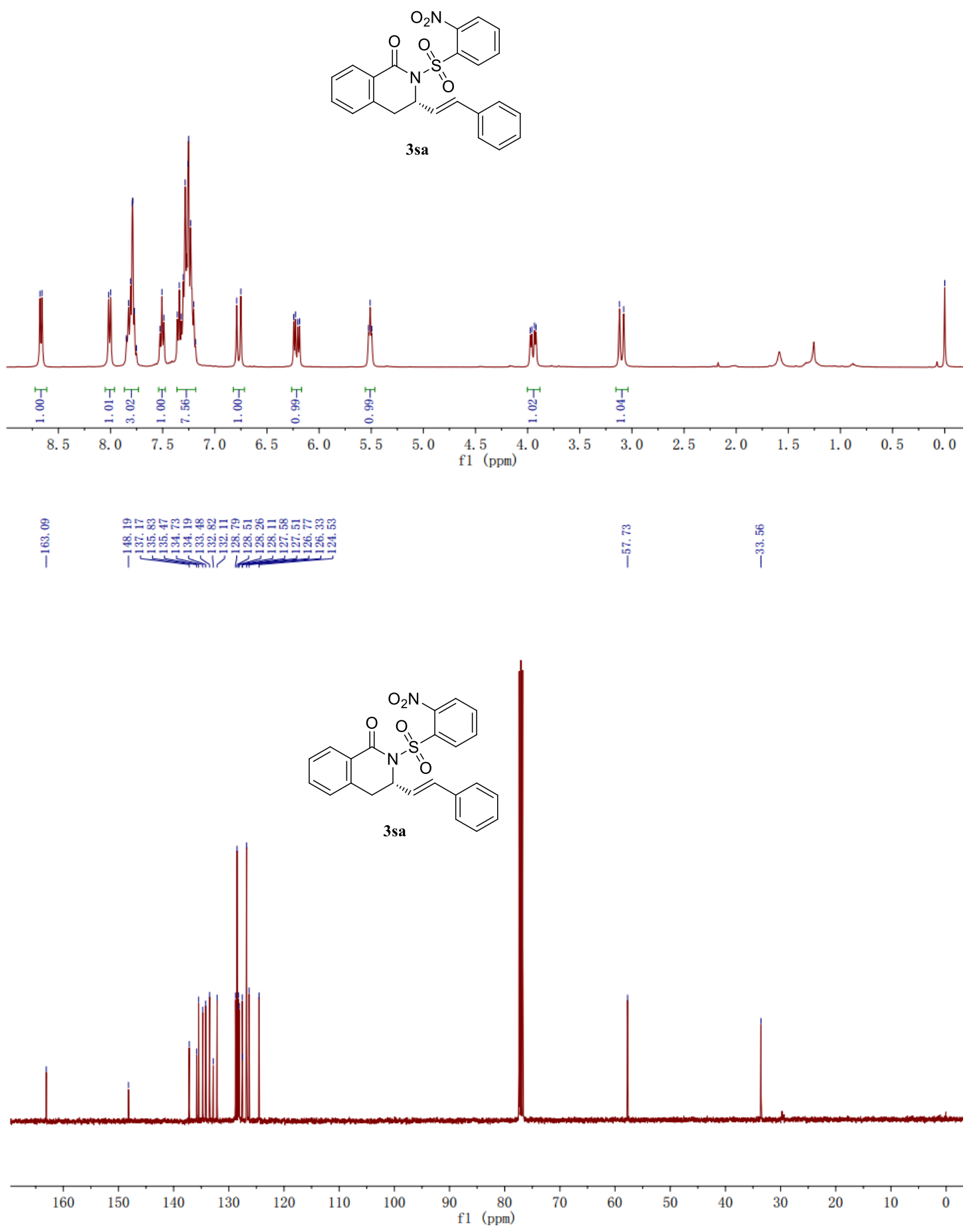

S46 
3 ta
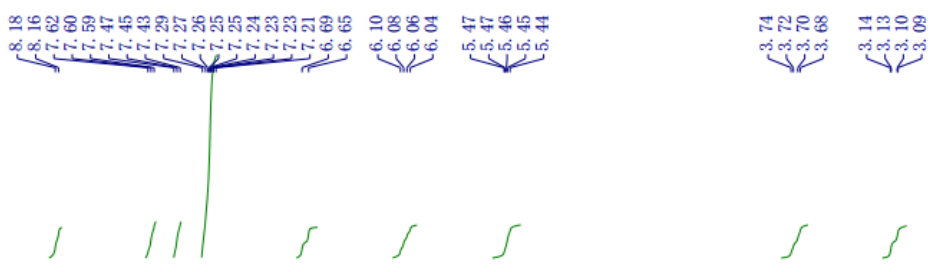

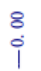
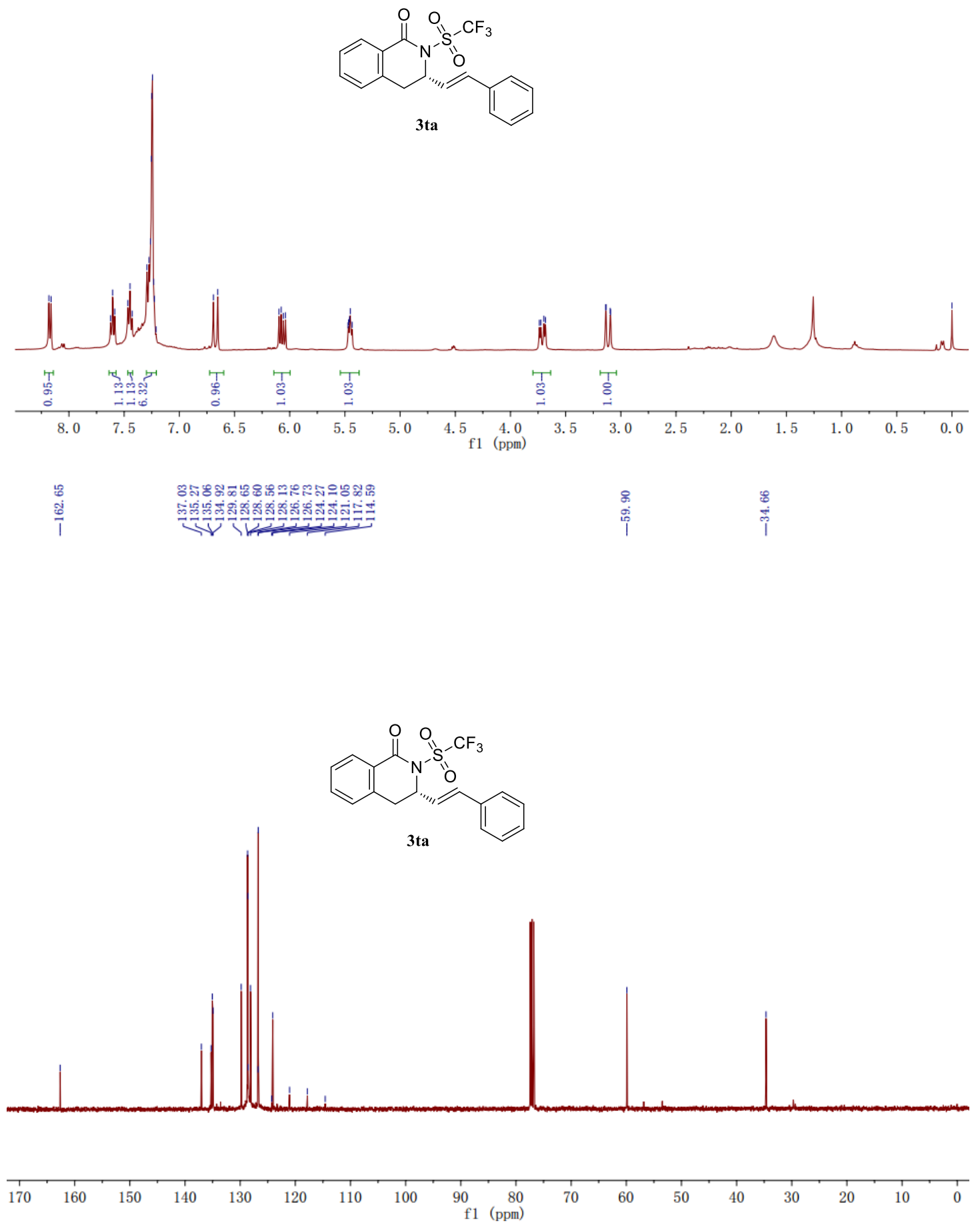

S47 
3ua
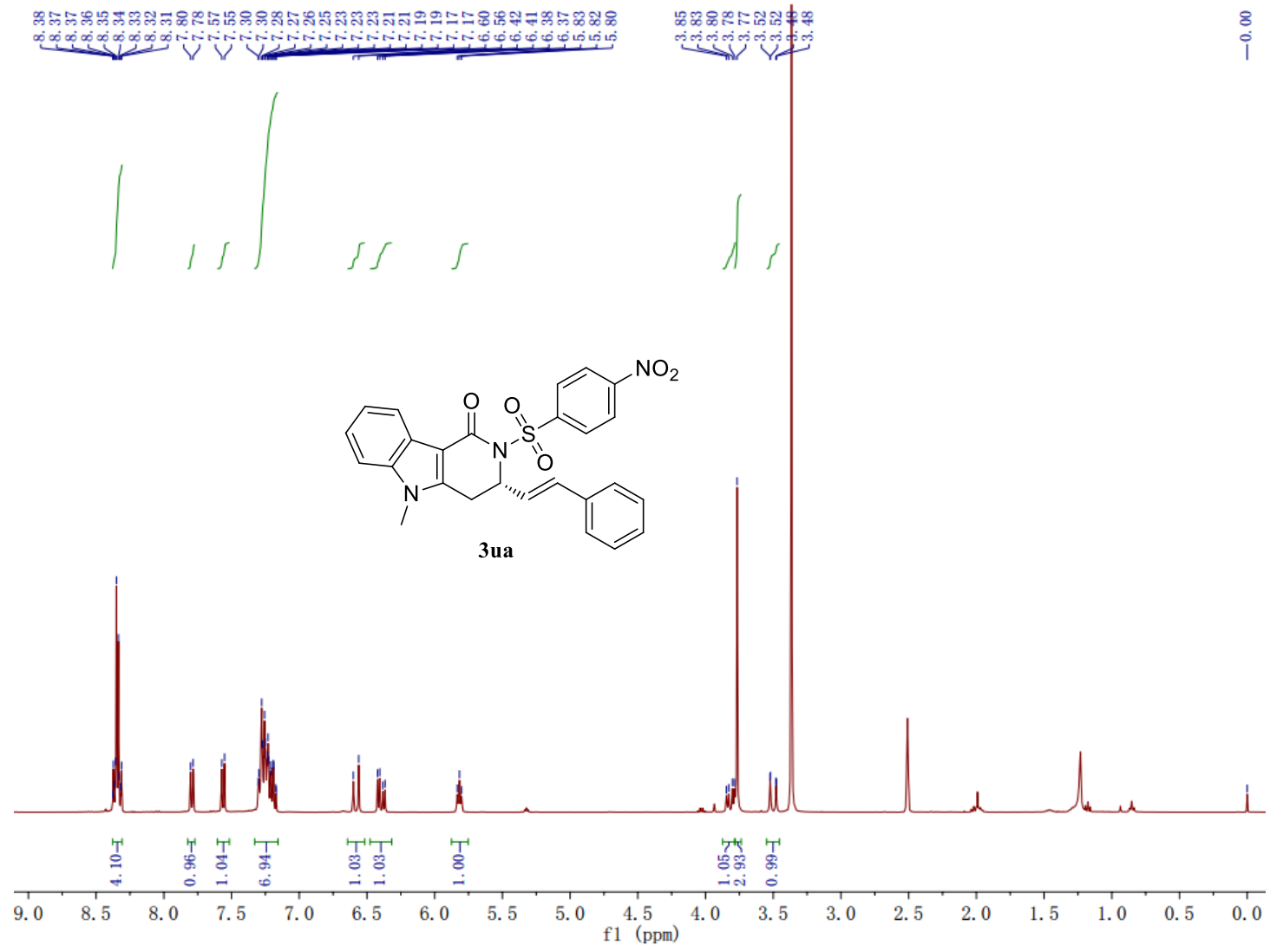

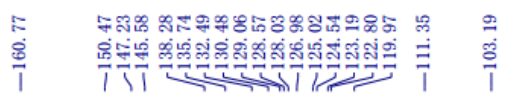
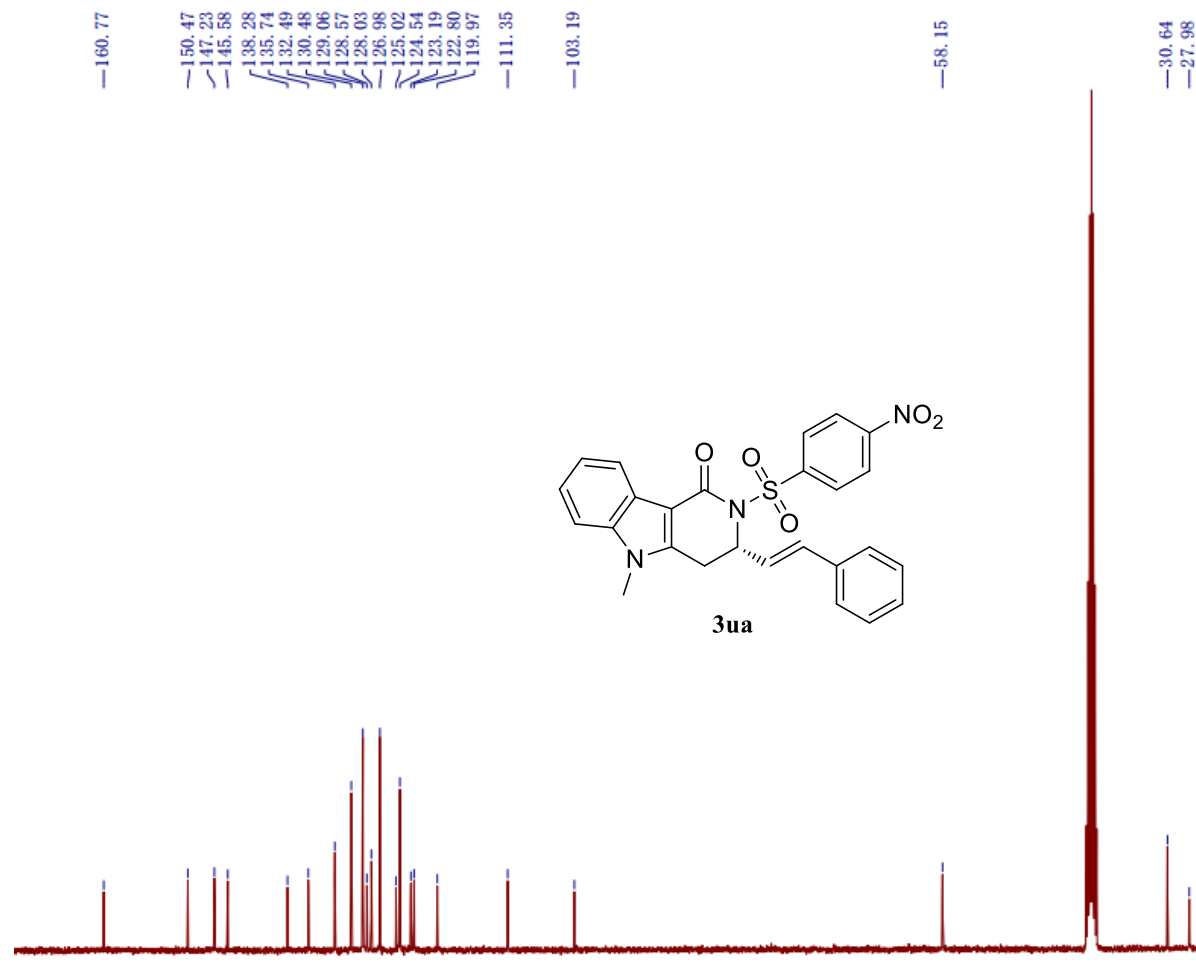

i)

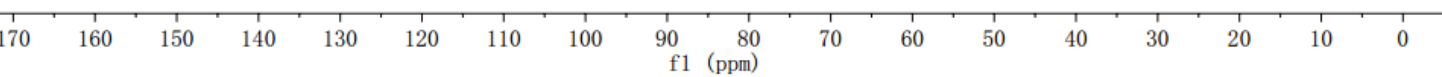


4

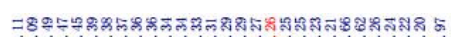

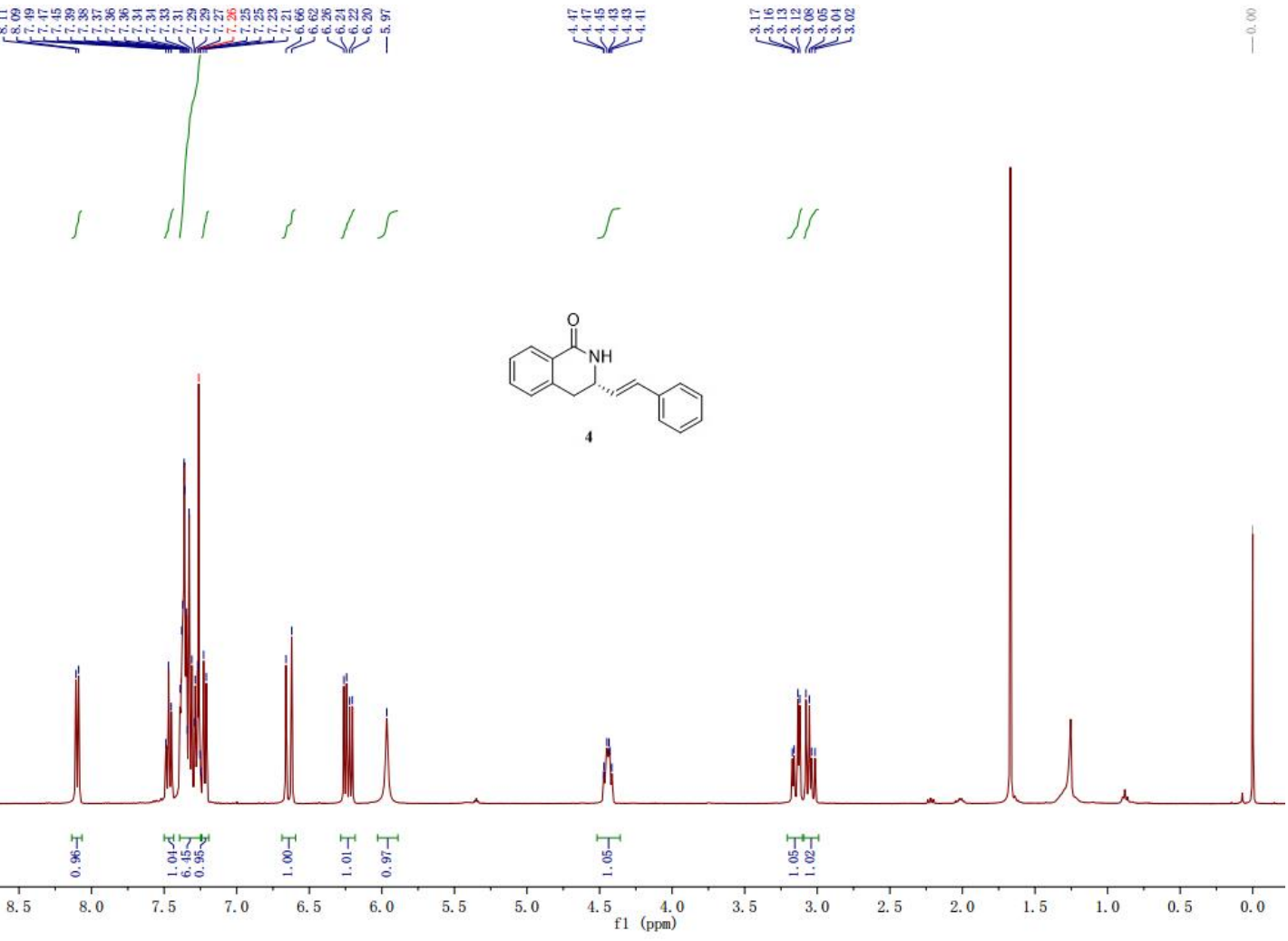

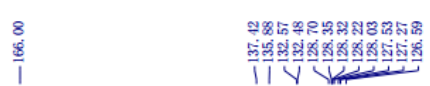

8
$\stackrel{8}{6}$
1
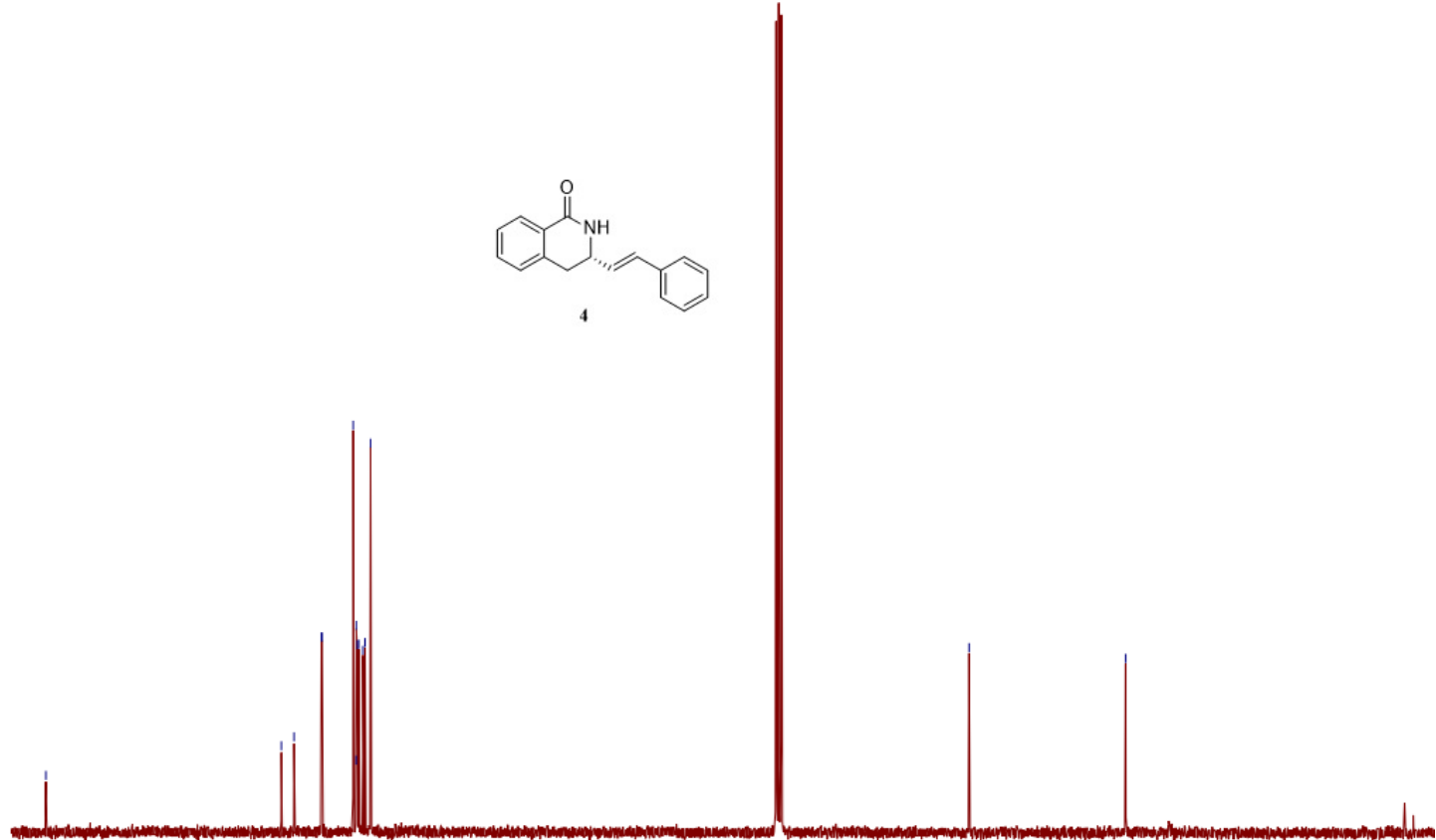

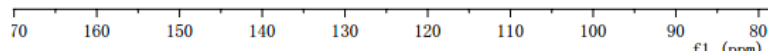

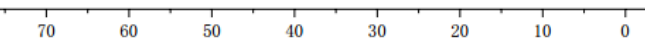


5
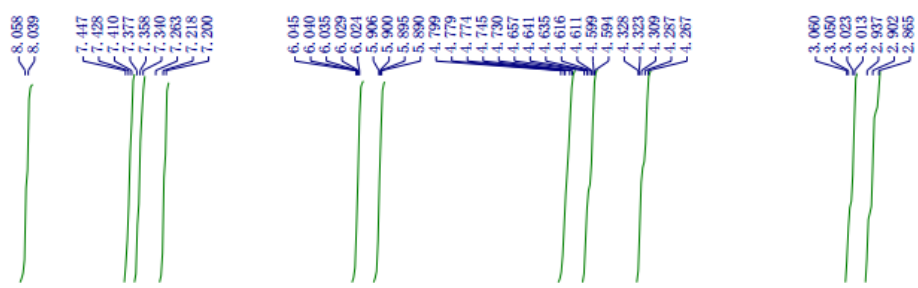

is
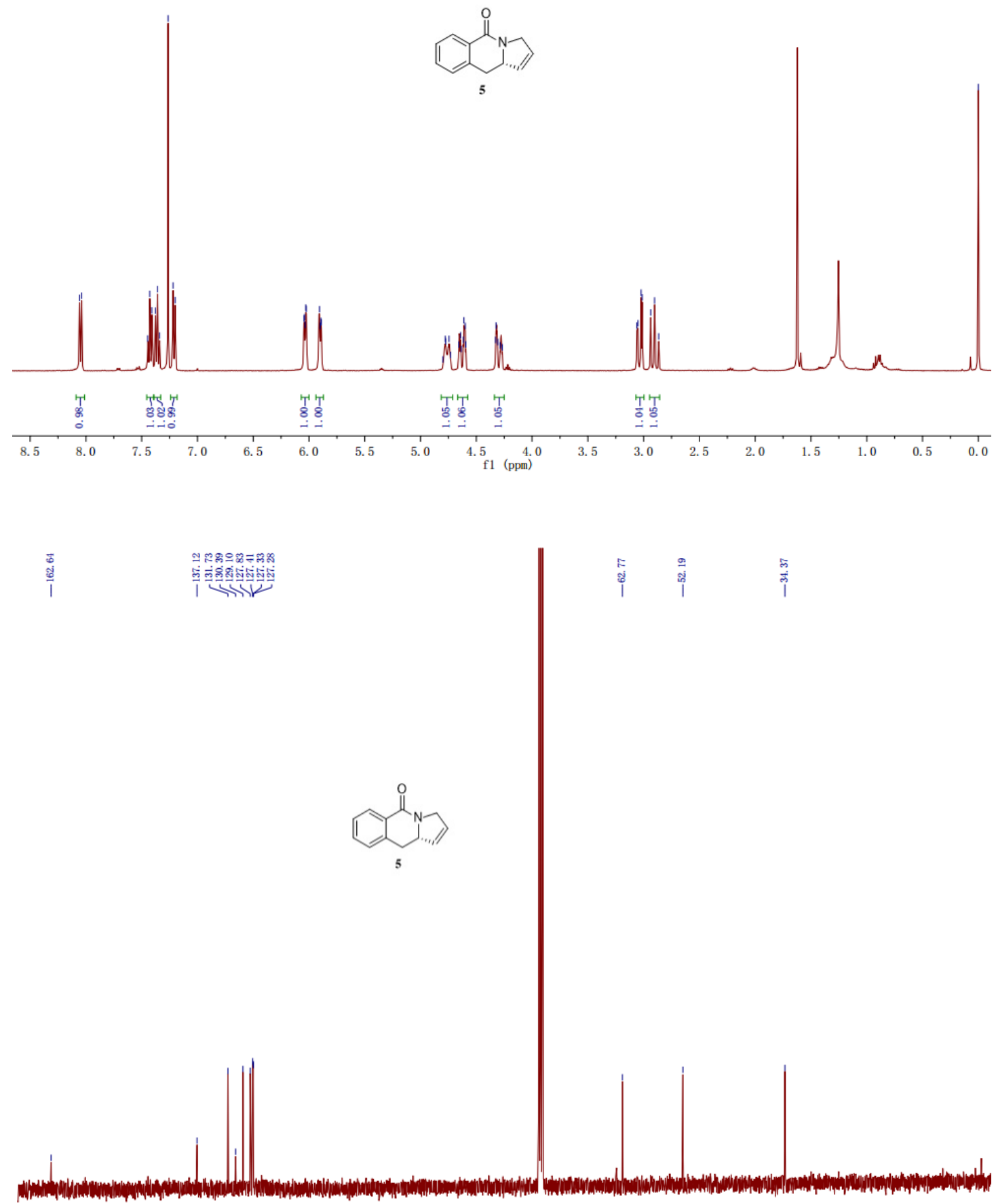

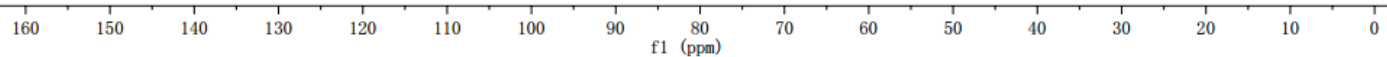



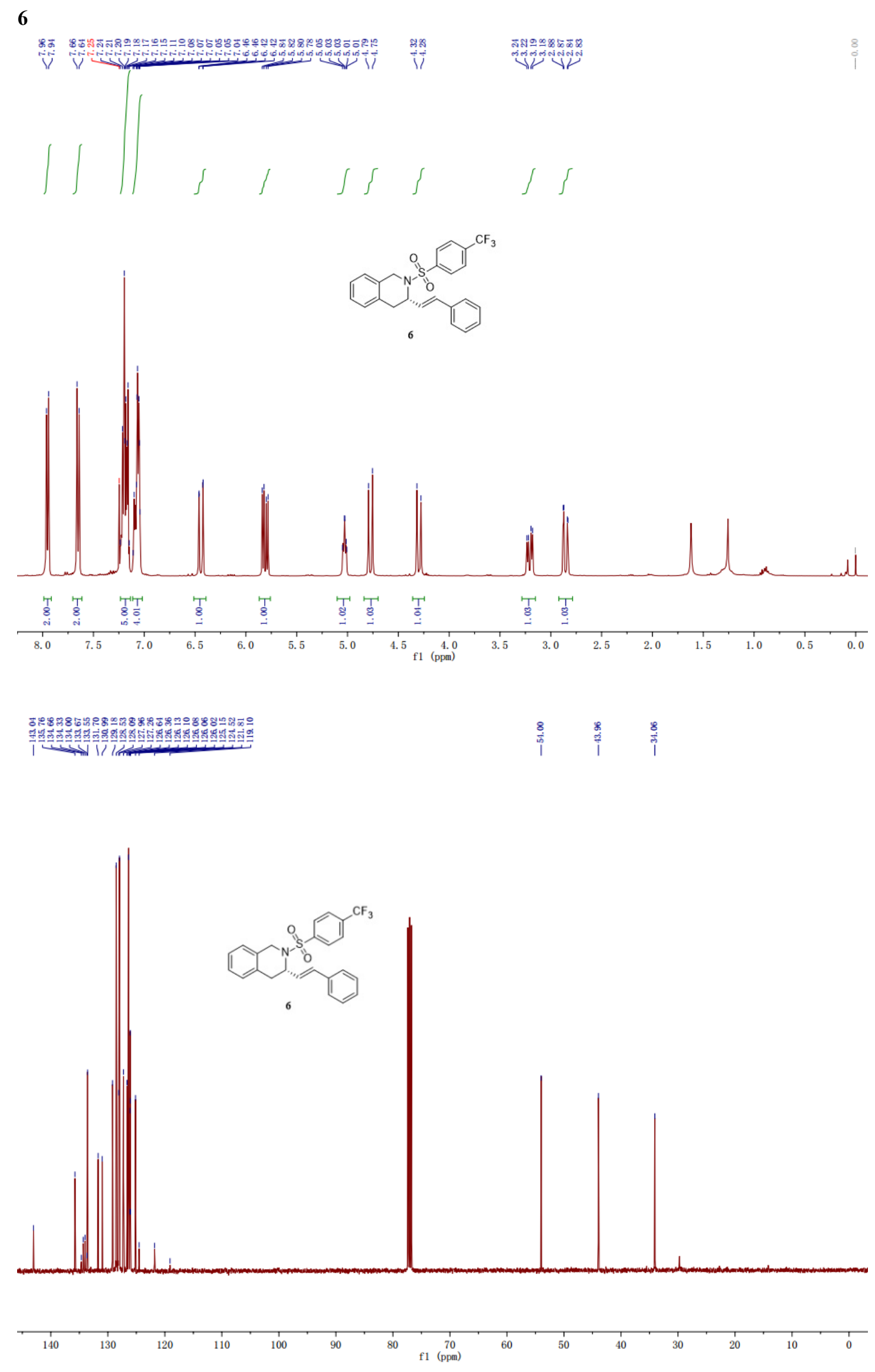

S51 
7

สำ หร๐

do

$\sqrt[4]{-688 \%}$

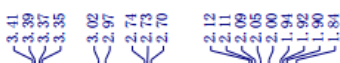

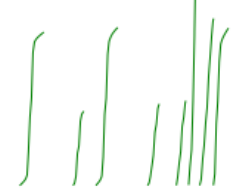

$\int \quad j \int 1 /$

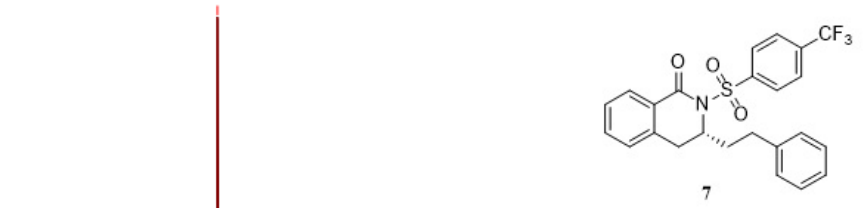

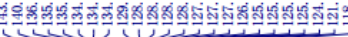
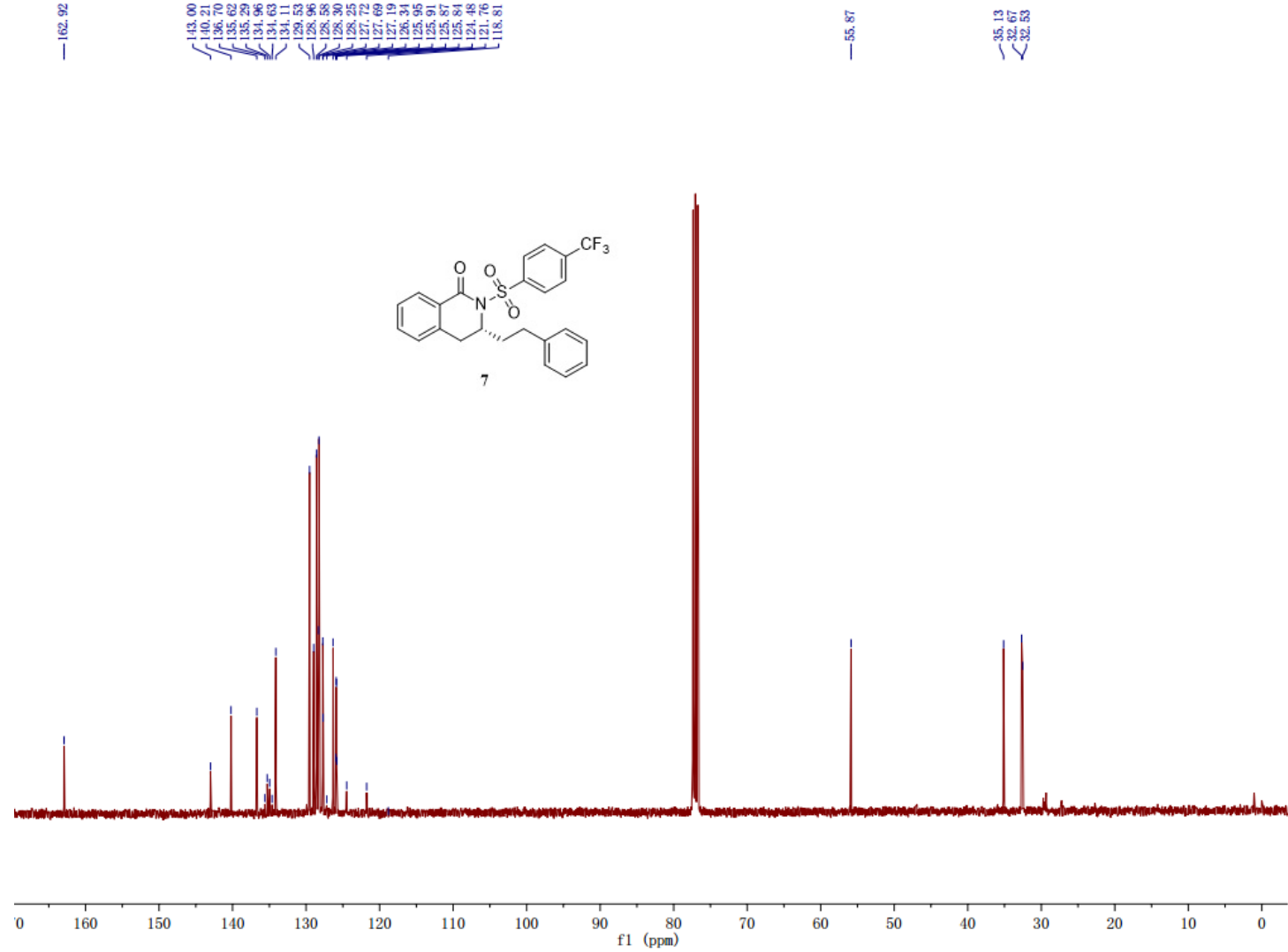


\section{HPLC Charts of related compounds}

$\mathrm{mV}$

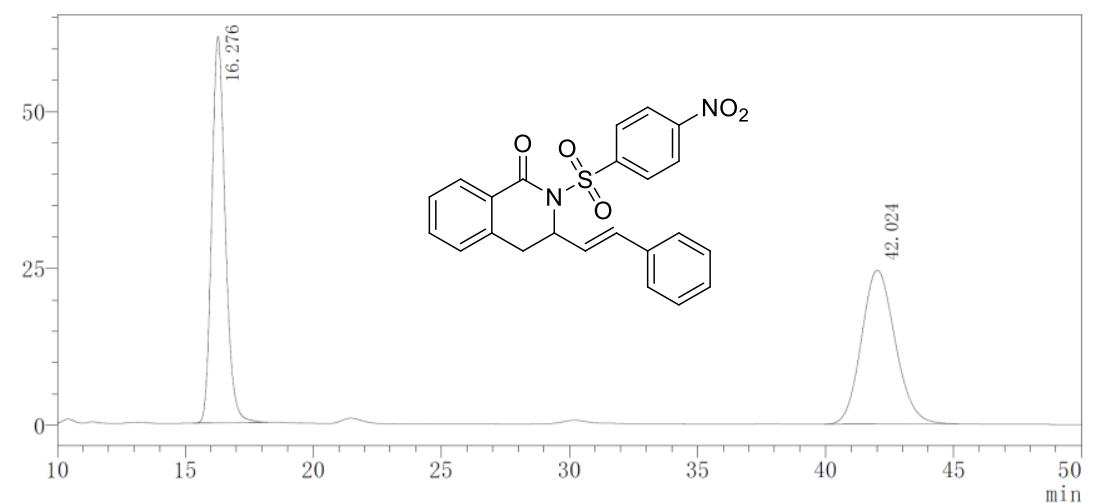

\begin{tabular}{|c|c|c|c|c|}
\hline Peak & RetTime $(\mathrm{min})$ & Area $(\mathrm{mV} * \mathrm{~S})$ & Height $(\mathrm{mV})$ & Area $(\%)$ \\
\hline 1 & 16.276 & 2251633 & 61578 & 50.050 \\
\hline 2 & 42.024 & 2247156 & 24525 & 49.950 \\
\hline
\end{tabular}

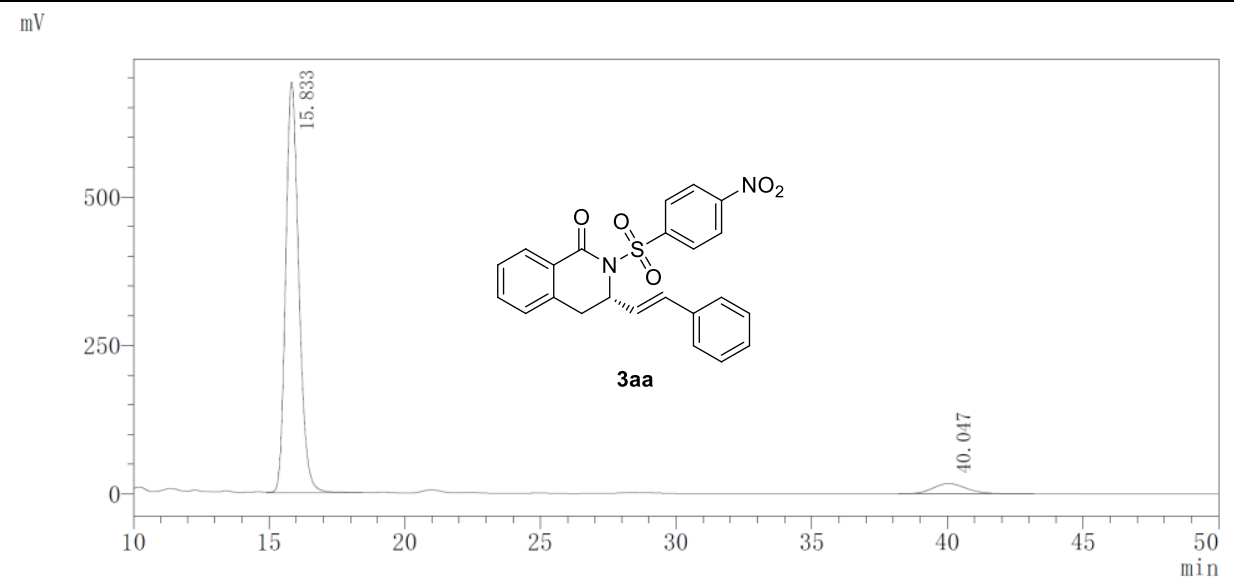

\begin{tabular}{|c|c|c|c|c|}
\hline Peak & RetTime $(\mathrm{min})$ & Area $(\mathrm{mV} * \mathrm{~S})$ & Height $(\mathrm{mV})$ & Area $(\%)$ \\
\hline 1 & 15.833 & 23228800 & 691035 & 94.052 \\
\hline 2 & 40.047 & 1469137 & 17226 & 5.948 \\
\hline
\end{tabular}

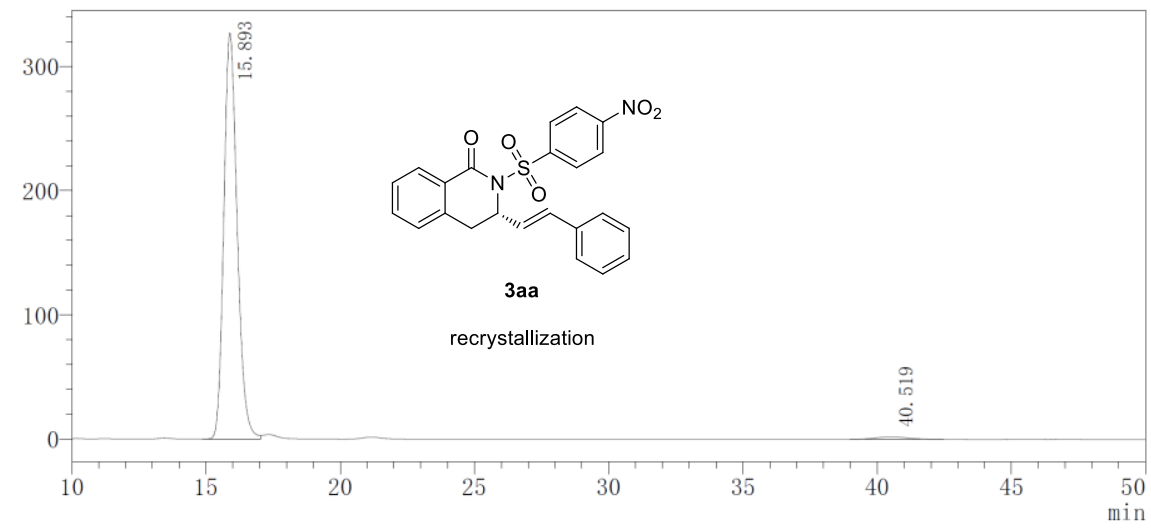

\begin{tabular}{|c|c|c|c|c|}
\hline Peak & RetTime $(\mathrm{min})$ & Area $(\mathrm{mV} * \mathrm{~S})$ & Height $(\mathrm{mV})$ & Area $(\%)$ \\
\hline 1 & 15.893 & 11284795 & 326788 & 98.456 \\
\hline 2 & 40.519 & 176916 & 2094 & 1.544 \\
\hline
\end{tabular}


3 ba

mV

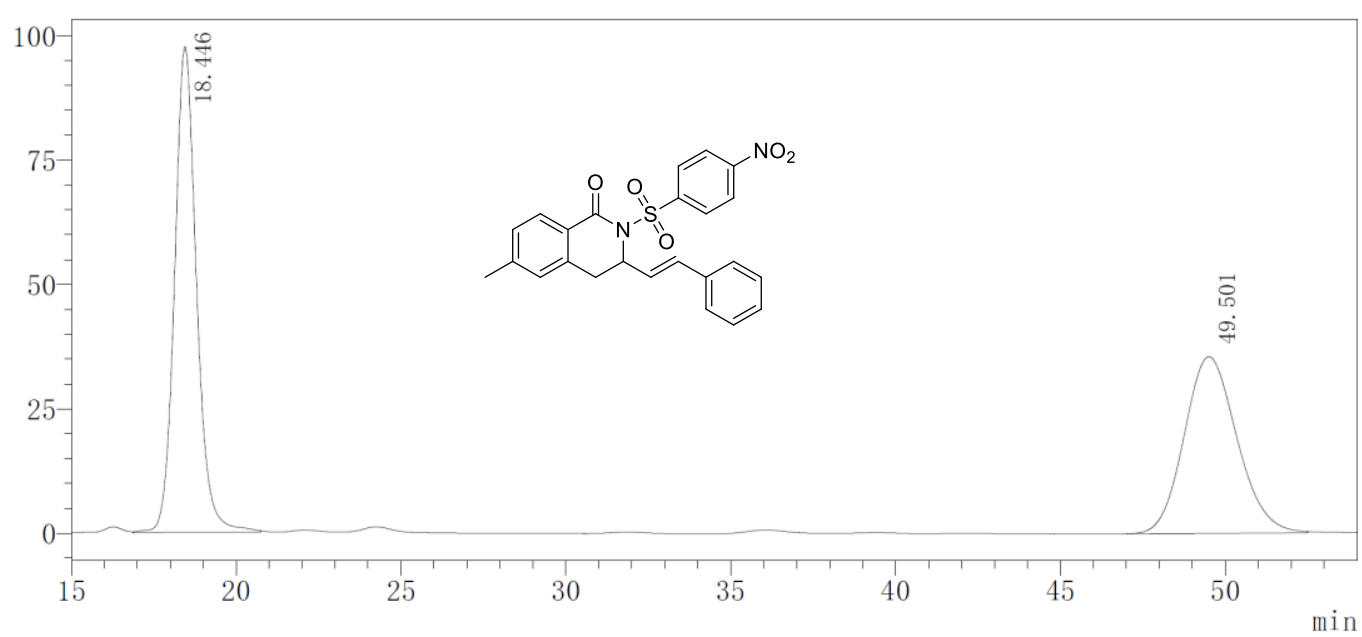

\begin{tabular}{|c|l|l|l|l|}
\hline $\begin{array}{c}\text { Peak } \\
\#\end{array}$ & $\begin{array}{c}\text { RetTime } \\
(\mathrm{min})\end{array}$ & $\begin{array}{c}\text { Area } \\
(\mathrm{mV} * \mathrm{~S})\end{array}$ & $\begin{array}{c}\text { Height } \\
(\mathrm{mV})\end{array}$ & $\begin{array}{c}\text { Area } \\
(\%)\end{array}$ \\
\hline 1 & 18.446 & 4522778 & 97640 & 53.343 \\
\hline 2 & 49.501 & 3955934 & 35548 & 46.657 \\
\hline
\end{tabular}

$\mathrm{mV}$

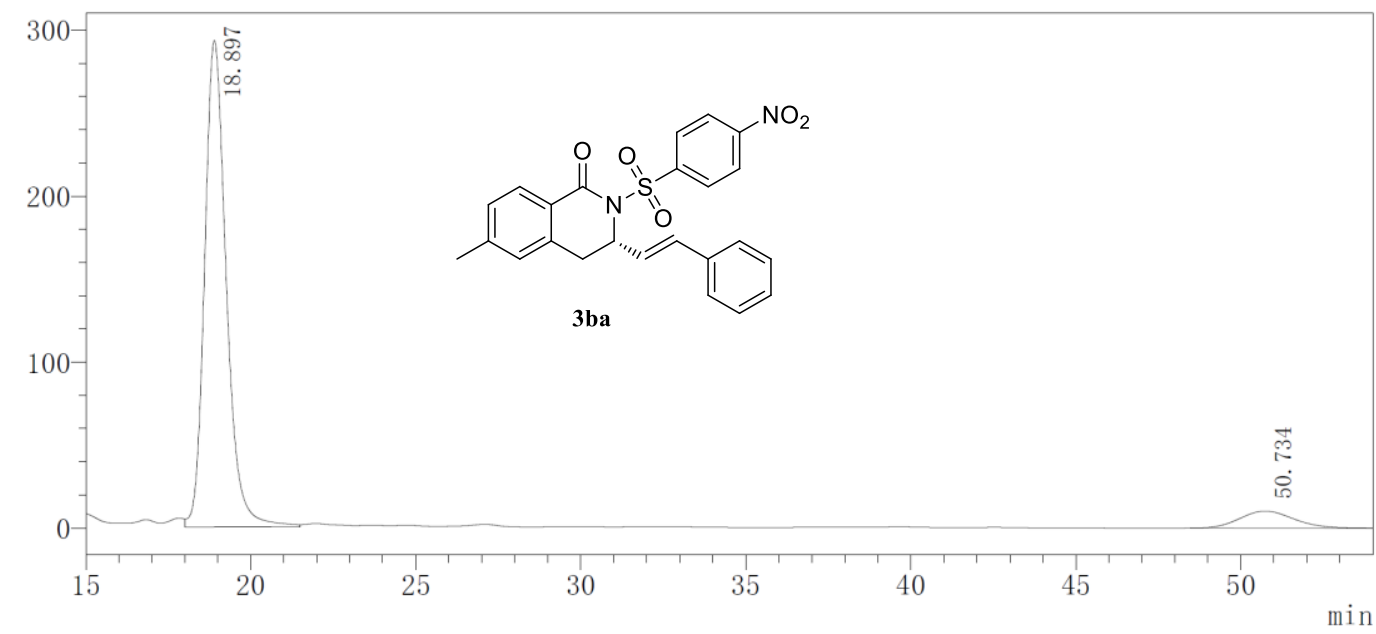

\begin{tabular}{|c|l|c|l|l|}
\hline $\begin{array}{c}\text { Peak } \\
\#\end{array}$ & $\begin{array}{c}\text { RetTime } \\
(\mathrm{min})\end{array}$ & $\begin{array}{c}\text { Area } \\
(\mathrm{mV} * \mathrm{~S})\end{array}$ & $\begin{array}{c}\text { Height } \\
(\mathrm{mV})\end{array}$ & $\begin{array}{c}\text { Area } \\
(\%)\end{array}$ \\
\hline 1 & 18.897 & 13358094 & 292889 & 91.896 \\
\hline 2 & 50.734 & 1177987 & 10344 & 8.104 \\
\hline
\end{tabular}


3 ca

$\mathrm{mV}$

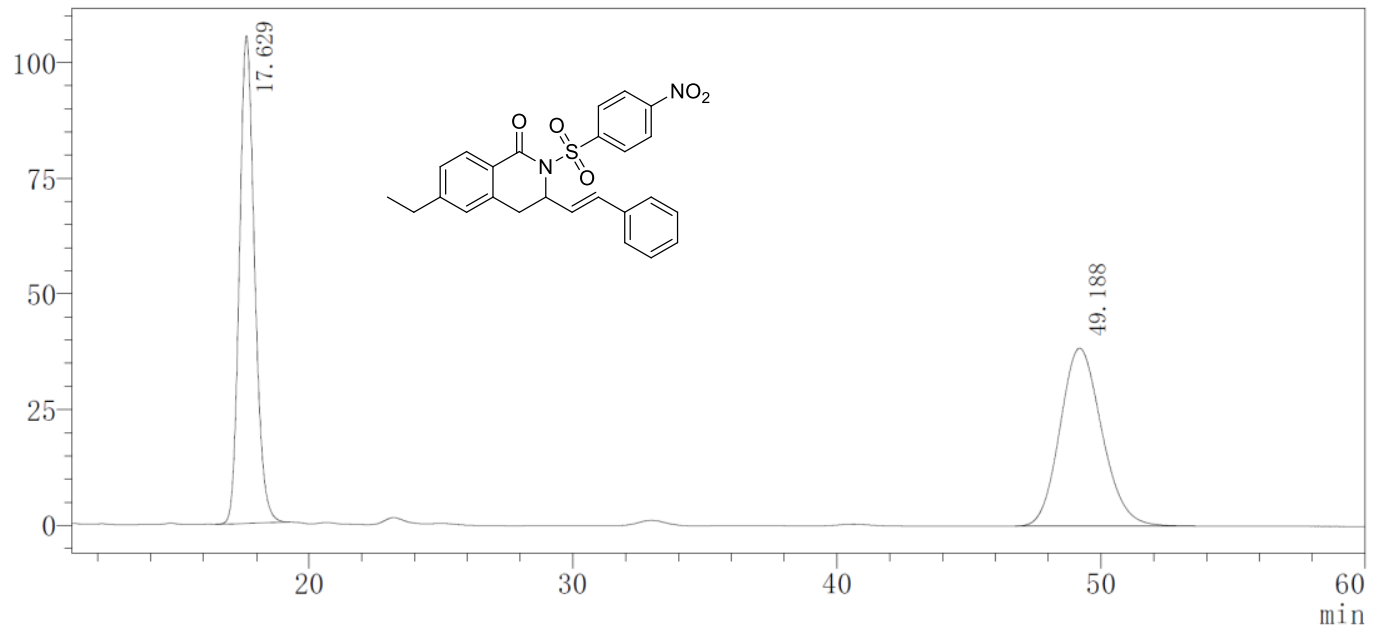

\begin{tabular}{|c|c|c|c|c|}
\hline $\begin{array}{c}\text { Peak } \\
\#\end{array}$ & $\begin{array}{c}\text { RetTime } \\
(\mathrm{min})\end{array}$ & $\begin{array}{c}\text { Area } \\
(\mathrm{mV} * \mathrm{~S})\end{array}$ & $\begin{array}{c}\text { Height } \\
(\mathrm{mV})\end{array}$ & $\begin{array}{c}\text { Area } \\
(\%)\end{array}$ \\
\hline 1 & 17.629 & 4226952 & 105400 & 49.892 \\
\hline 2 & 49.188 & 4245272 & 38485 & 50.108 \\
\hline
\end{tabular}

$\mathrm{mV}$

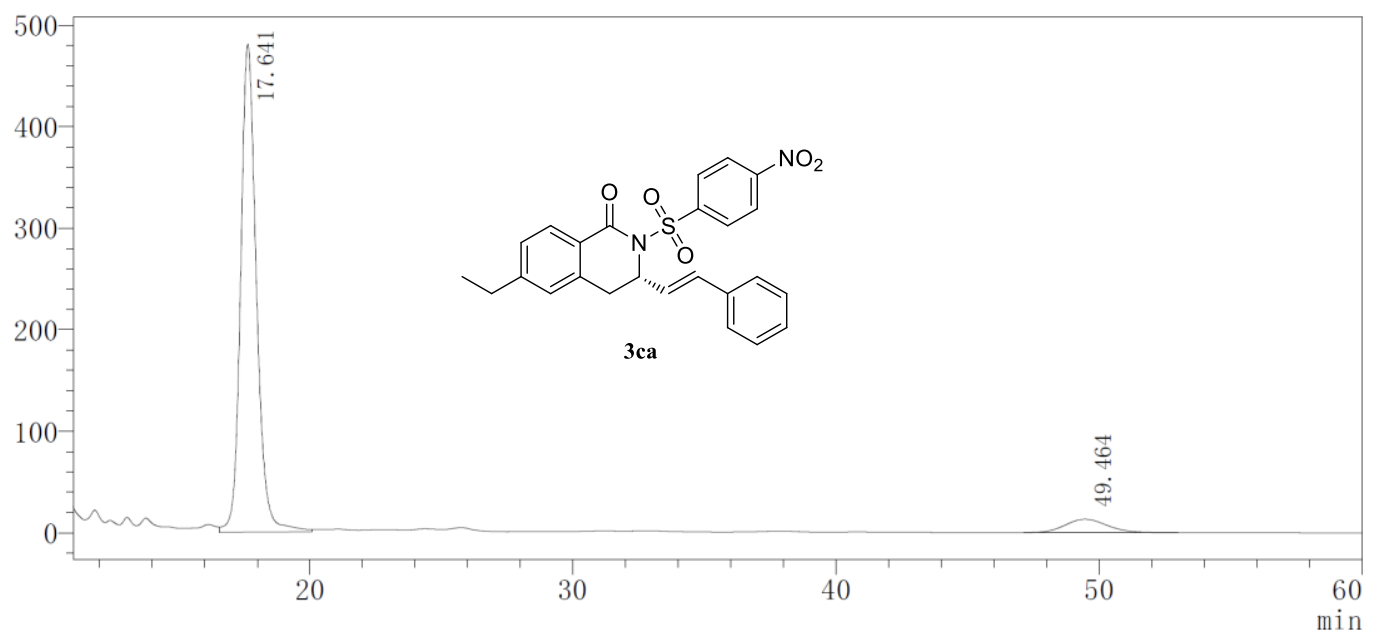

\begin{tabular}{|c|l|l|l|l|}
\hline $\begin{array}{c}\text { Peak } \\
\#\end{array}$ & $\begin{array}{c}\text { RetTime } \\
(\mathrm{min})\end{array}$ & $\begin{array}{c}\text { Area } \\
(\mathrm{mV} * \mathrm{~S})\end{array}$ & $\begin{array}{c}\text { Height } \\
(\mathrm{mV})\end{array}$ & $\begin{array}{c}\text { Area } \\
(\%)\end{array}$ \\
\hline 1 & 17.641 & 20121656 & 480449 & 93.171 \\
\hline 2 & 49.464 & 1474866 & 13252 & 6.829 \\
\hline
\end{tabular}


3da

mV

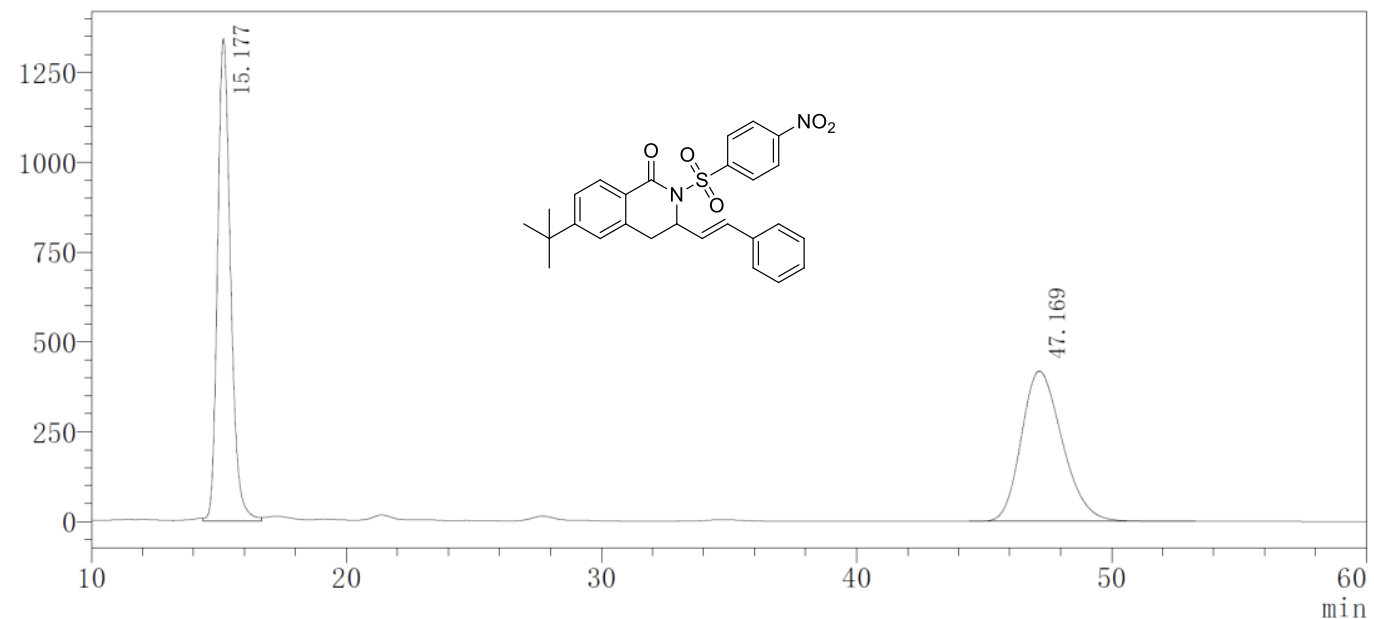

\begin{tabular}{|c|c|c|c|c|}
\hline $\begin{array}{c}\text { Peak } \\
\#\end{array}$ & $\begin{array}{c}\text { RetTime } \\
(\mathrm{min})\end{array}$ & $\begin{array}{c}\text { Area } \\
(\mathrm{mV} * \mathrm{~S})\end{array}$ & $\begin{array}{c}\text { Height } \\
(\mathrm{mV})\end{array}$ & $\begin{array}{c}\text { Area } \\
(\%)\end{array}$ \\
\hline 1 & 15.177 & 47904940 & 1343093 & 50.603 \\
\hline 2 & 47.169 & 46762483 & 418381 & 49.397 \\
\hline
\end{tabular}

$\mathrm{mV}$

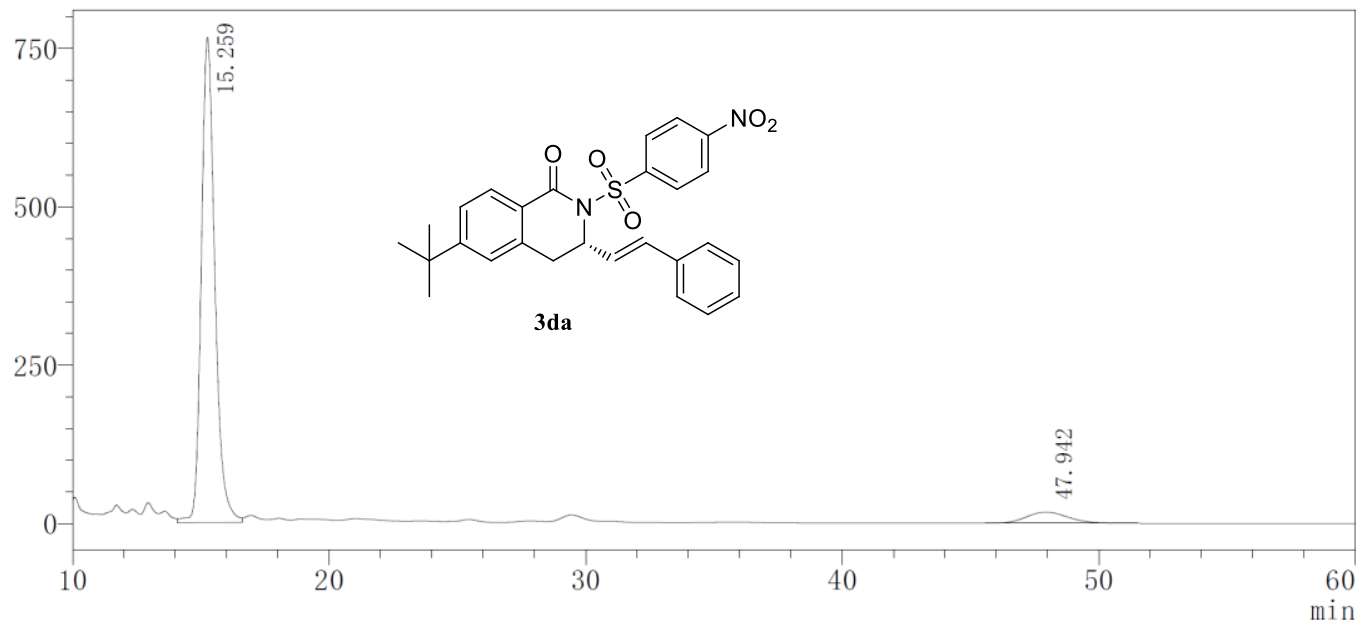

\begin{tabular}{|c|l|l|l|l|}
\hline $\begin{array}{c}\text { Peak } \\
\#\end{array}$ & $\begin{array}{c}\text { RetTime } \\
(\mathrm{min})\end{array}$ & $\begin{array}{c}\text { Area } \\
(\mathrm{mV} * \mathrm{~S})\end{array}$ & $\begin{array}{c}\text { Height } \\
(\mathrm{mV})\end{array}$ & $\begin{array}{c}\text { Area } \\
(\%)\end{array}$ \\
\hline 1 & 15.259 & 28137748 & 765876 & 93.270 \\
\hline 2 & 47.942 & 2030151 & 17885 & 6.730 \\
\hline
\end{tabular}


3 ea

$\mathrm{mV}$

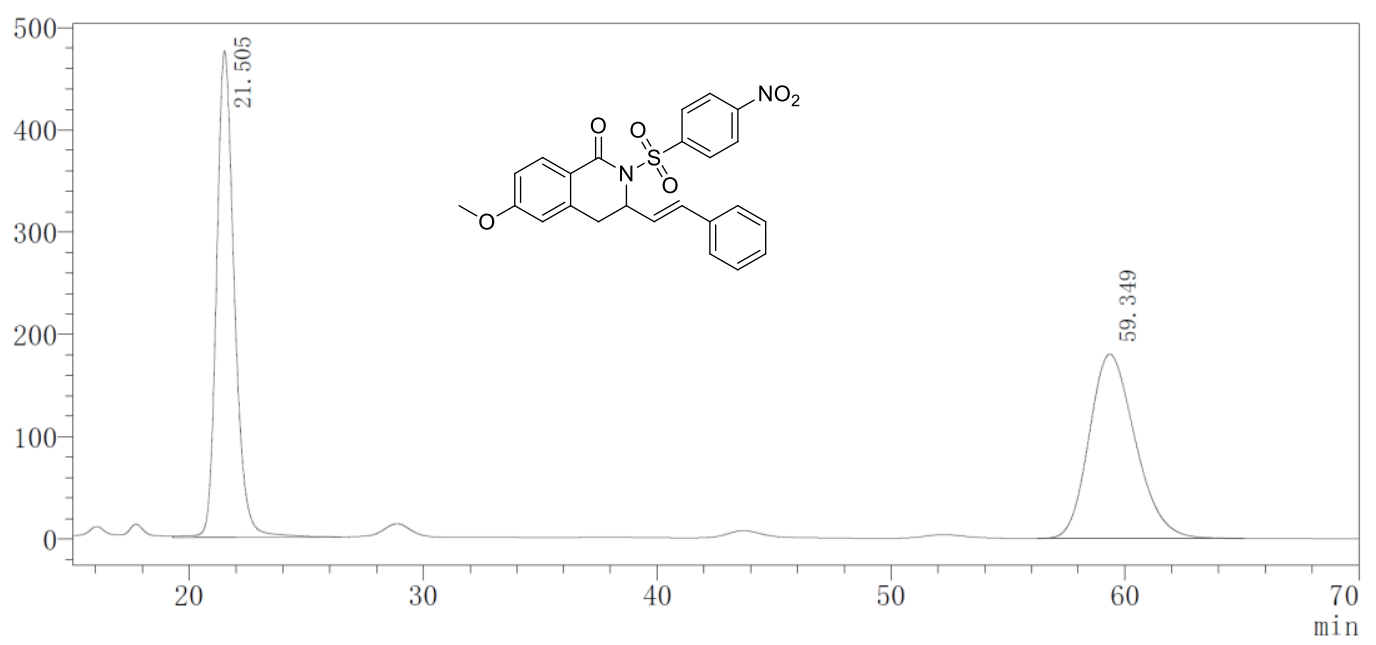

\begin{tabular}{|c|l|c|c|c|}
\hline $\begin{array}{c}\text { Peak } \\
\#\end{array}$ & $\begin{array}{c}\text { RetTime } \\
(\mathrm{min})\end{array}$ & $\begin{array}{c}\text { Area } \\
(\mathrm{mV} * \mathrm{~S})\end{array}$ & $\begin{array}{c}\text { Height } \\
(\mathrm{mV})\end{array}$ & $\begin{array}{c}\text { Area } \\
(\%)\end{array}$ \\
\hline 1 & 21.505 & 25204987 & 475367 & 50.540 \\
\hline 2 & 59.349 & 24666188 & 180081 & 49.460 \\
\hline
\end{tabular}

$\mathrm{mV}$

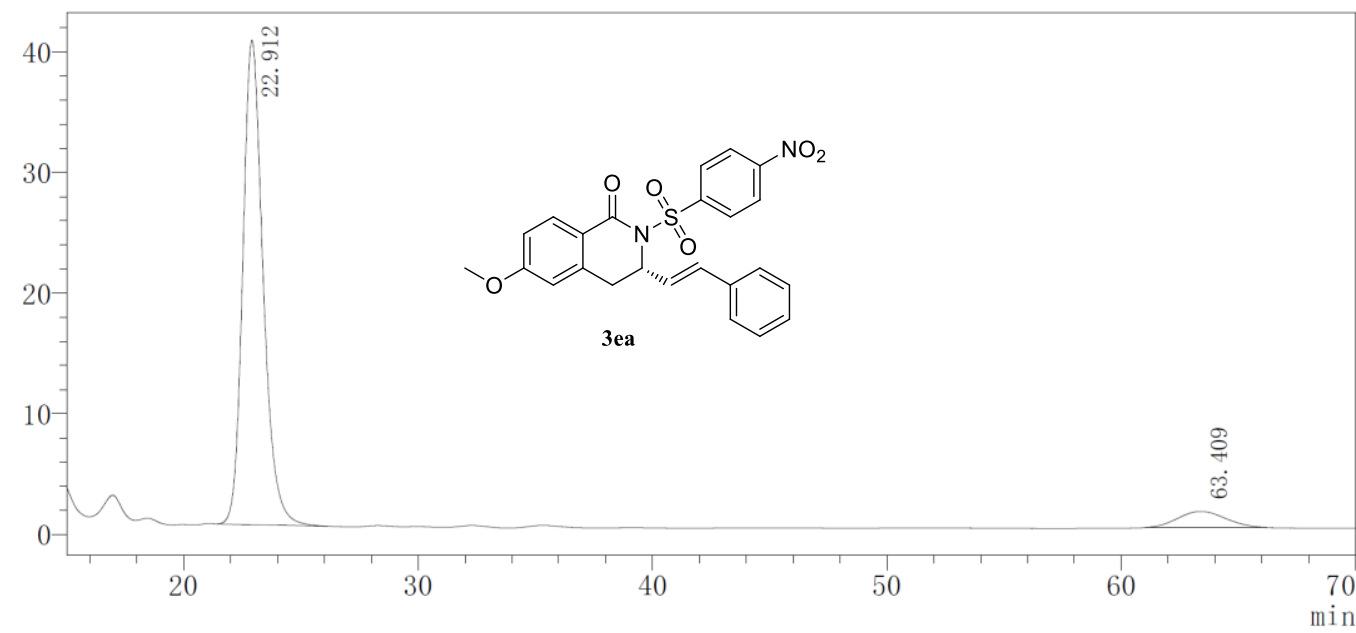

\begin{tabular}{|c|l|l|l|l|}
\hline $\begin{array}{c}\text { Peak } \\
\#\end{array}$ & $\begin{array}{c}\text { RetTime } \\
(\mathrm{min})\end{array}$ & $\begin{array}{c}\text { Area } \\
(\mathrm{mV} * \mathrm{~S})\end{array}$ & $\begin{array}{c}\text { Height } \\
(\mathrm{mV})\end{array}$ & $\begin{array}{c}\text { Area } \\
(\%)\end{array}$ \\
\hline 1 & 22.912 & 2421244 & 40167 & 92.703 \\
\hline 2 & 63.409 & 190572 & 1346 & 7.297 \\
\hline
\end{tabular}




\section{3fa}

uV

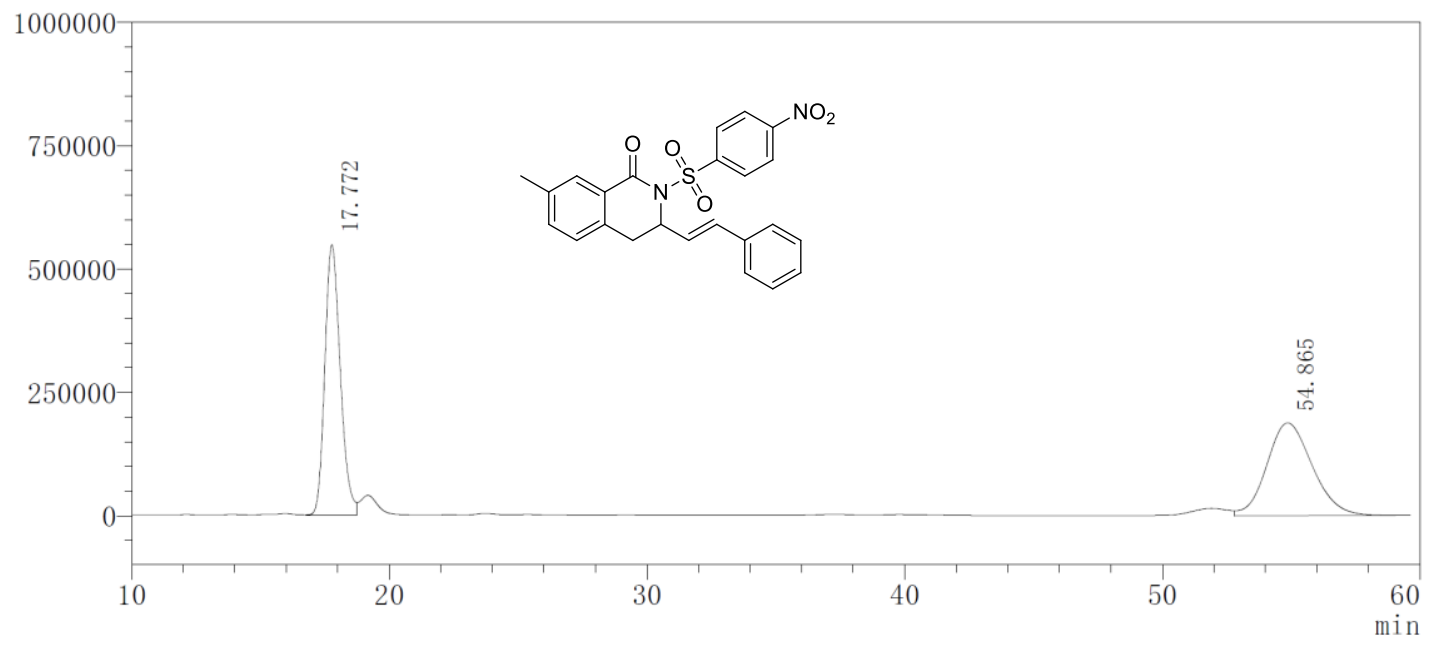

\begin{tabular}{|c|c|c|c|c|}
\hline $\begin{array}{c}\text { Peak } \\
\#\end{array}$ & $\begin{array}{c}\text { RetTime } \\
(\mathrm{min})\end{array}$ & $\begin{array}{c}\text { Area } \\
(\mathrm{mV} * \mathrm{~S})\end{array}$ & $\begin{array}{c}\text { Height } \\
(\mathrm{mV})\end{array}$ & $\begin{array}{c}\text { Area } \\
(\%)\end{array}$ \\
\hline 1 & 17.772 & 22829359 & 548168 & 49.745 \\
\hline 2 & 54.865 & 23063141 & 187926 & 50.255 \\
\hline
\end{tabular}

$\mathrm{uV}$

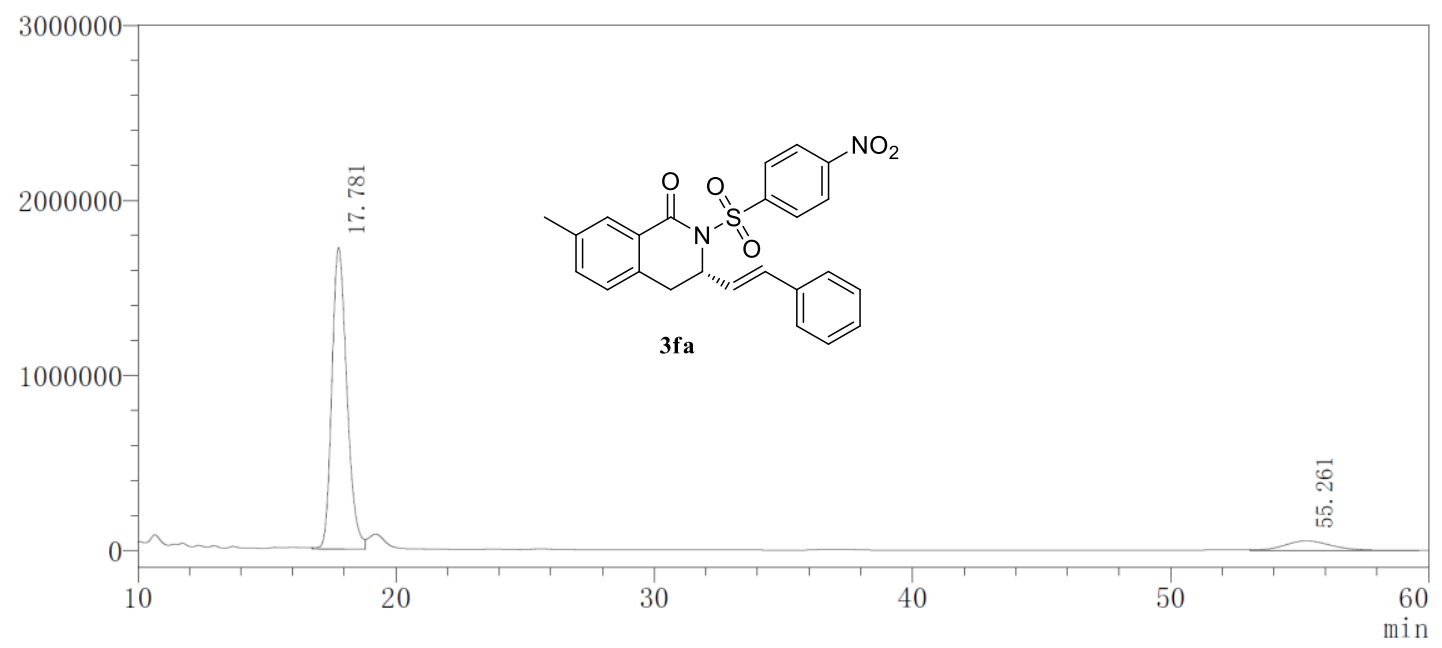

\begin{tabular}{|c|l|l|l|l|}
\hline $\begin{array}{c}\text { Peak } \\
\#\end{array}$ & $\begin{array}{c}\text { RetTime } \\
(\mathrm{min})\end{array}$ & $\begin{array}{c}\text { Area } \\
(\mathrm{mV} * \mathrm{~S})\end{array}$ & $\begin{array}{c}\text { Height } \\
(\mathrm{mV})\end{array}$ & $\begin{array}{c}\text { Area } \\
(\%)\end{array}$ \\
\hline 1 & 17.781 & 69273374 & 1722162 & 91.158 \\
\hline 2 & 55.261 & 6719280 & 54377 & 8.842 \\
\hline
\end{tabular}




\section{3ga}

$\mathrm{mV}$

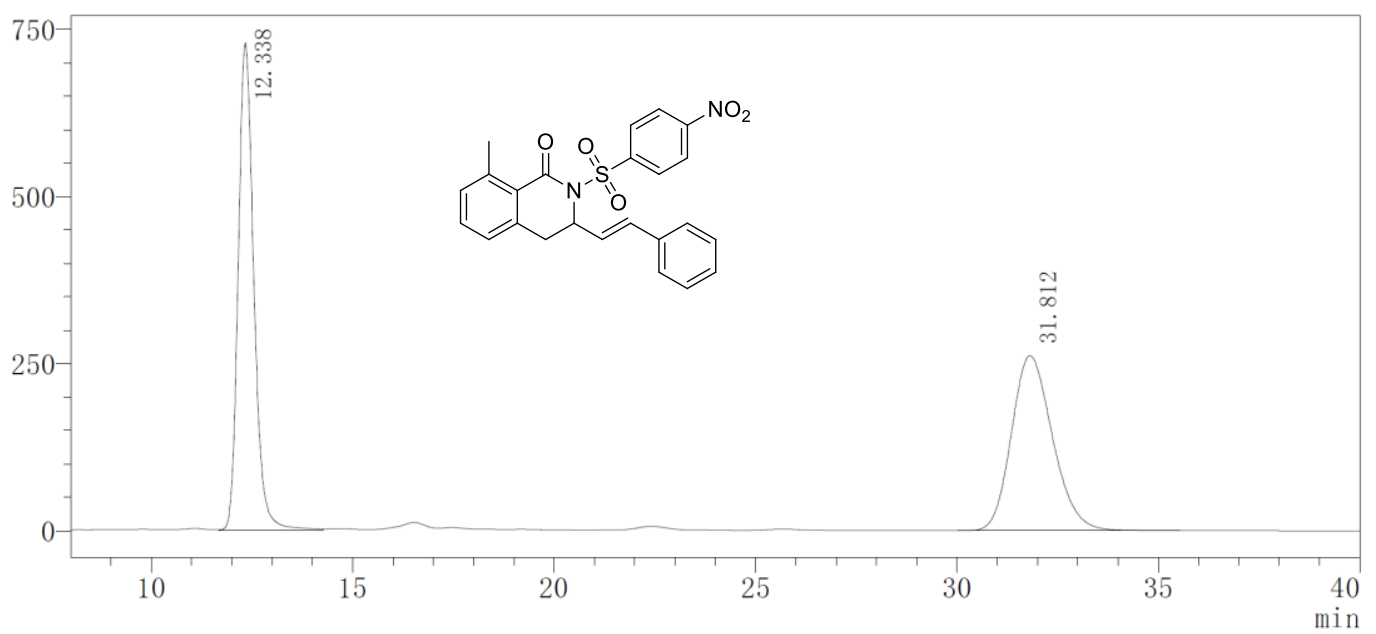

\begin{tabular}{|c|c|c|c|c|}
\hline $\begin{array}{c}\text { Peak } \\
\#\end{array}$ & $\begin{array}{c}\text { RetTime } \\
(\mathrm{min})\end{array}$ & $\begin{array}{c}\text { Area } \\
(\mathrm{mV} * \mathrm{~S})\end{array}$ & $\begin{array}{c}\text { Height } \\
(\mathrm{mV})\end{array}$ & $\begin{array}{c}\text { Area } \\
(\%)\end{array}$ \\
\hline 1 & 12.338 & 19705468 & 729145 & 51.467 \\
\hline 2 & 31.812 & 18582126 & 261715 & 48.533 \\
\hline
\end{tabular}

$\mathrm{mV}$

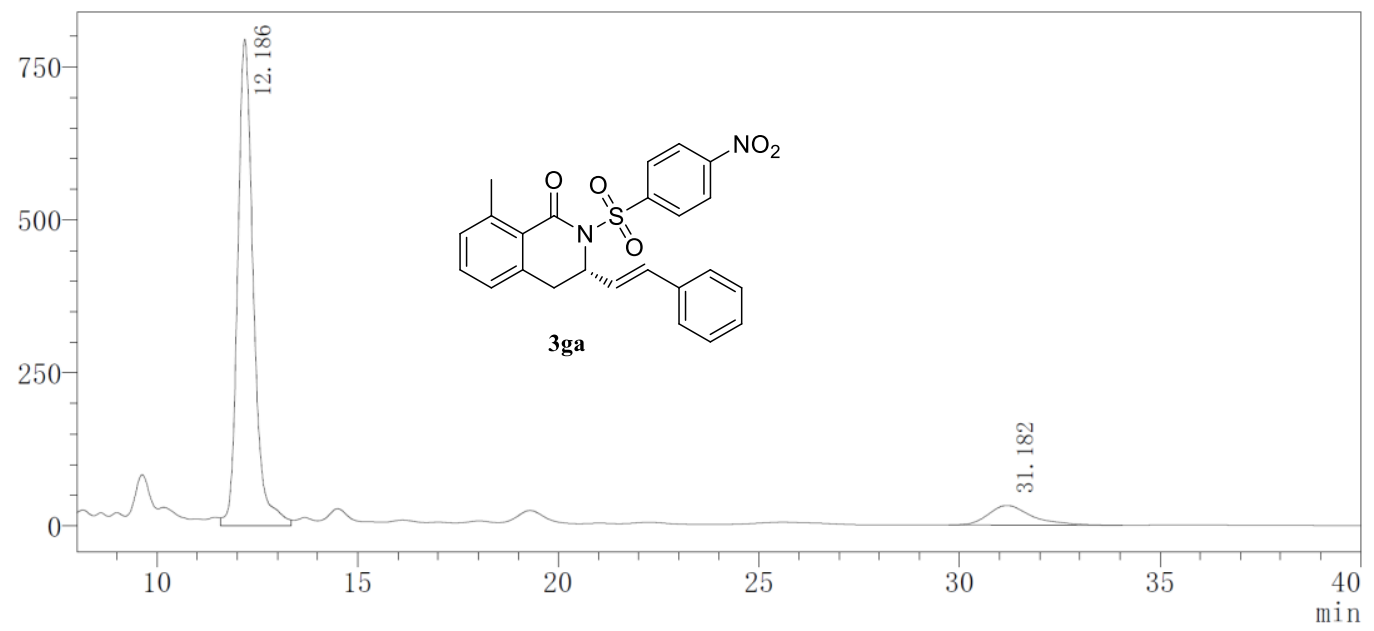

\begin{tabular}{|c|c|c|c|c|}
\hline $\begin{array}{c}\text { Peak } \\
\#\end{array}$ & $\begin{array}{c}\text { RetTime } \\
(\mathrm{min})\end{array}$ & $\begin{array}{c}\text { Area } \\
(\mathrm{mV} * \mathrm{~S})\end{array}$ & $\begin{array}{c}\text { Height } \\
(\mathrm{mV})\end{array}$ & $\begin{array}{c}\text { Area } \\
(\%)\end{array}$ \\
\hline 1 & 12.186 & 21608226 & 794703 & 90.072 \\
\hline 2 & 31.182 & 2381785 & 31885 & 9.928 \\
\hline
\end{tabular}


3ha

$\mathrm{mV}$

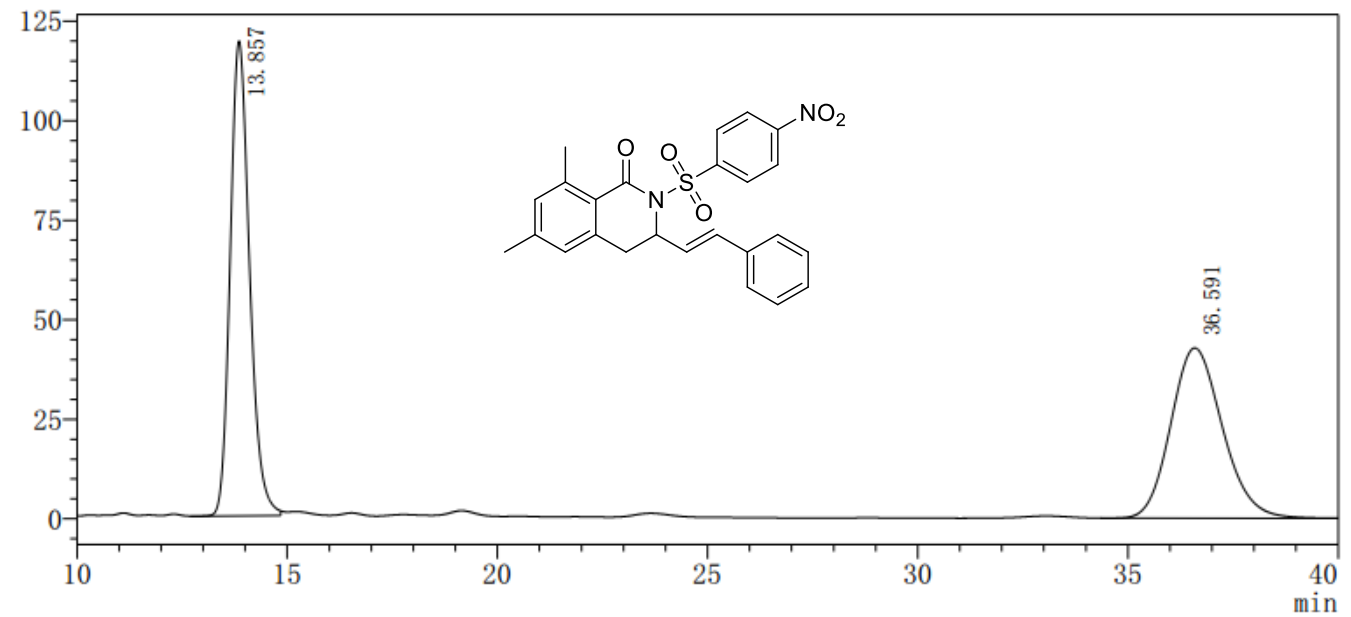

\begin{tabular}{|c|l|l|l|l|}
\hline $\begin{array}{c}\text { Peak } \\
\#\end{array}$ & $\begin{array}{c}\text { RetTime } \\
(\mathrm{min})\end{array}$ & $\begin{array}{c}\text { Area } \\
(\mathrm{mV} * \mathrm{~S})\end{array}$ & $\begin{array}{c}\text { Height } \\
(\mathrm{mV})\end{array}$ & $\begin{array}{c}\text { Area } \\
(\%)\end{array}$ \\
\hline 1 & 13.857 & 3830832 & 119276 & 51.119 \\
\hline 2 & 36.591 & 3663089 & 42791 & 48.881 \\
\hline
\end{tabular}

mV

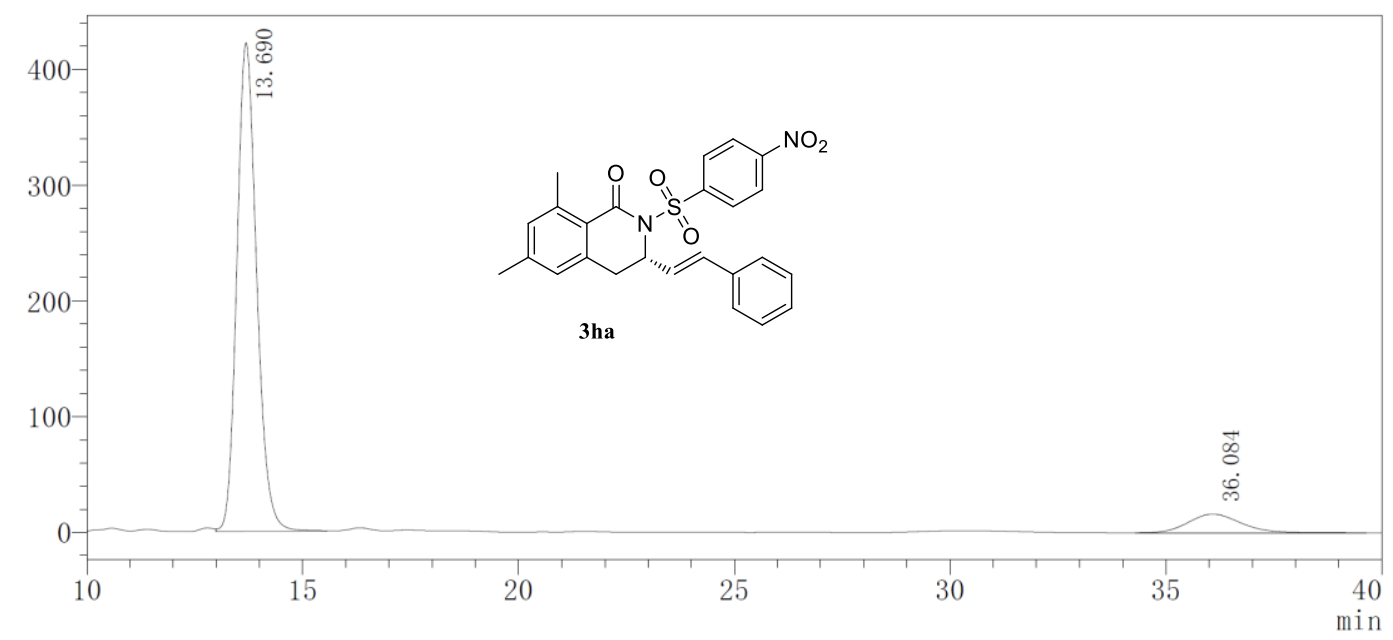

\begin{tabular}{|c|l|l|l|l|}
\hline $\begin{array}{c}\text { Peak } \\
\#\end{array}$ & $\begin{array}{c}\text { RetTime } \\
(\mathrm{min})\end{array}$ & $\begin{array}{c}\text { Area } \\
(\mathrm{mV} * \mathrm{~S})\end{array}$ & $\begin{array}{c}\text { Height } \\
(\mathrm{mV})\end{array}$ & $\begin{array}{c}\text { Area } \\
(\%)\end{array}$ \\
\hline 1 & 13.690 & 13497390 & 422204 & 90.796 \\
\hline 2 & 36.084 & 1368243 & 16083 & 9.204 \\
\hline
\end{tabular}


3 ia

$\mathrm{mV}$

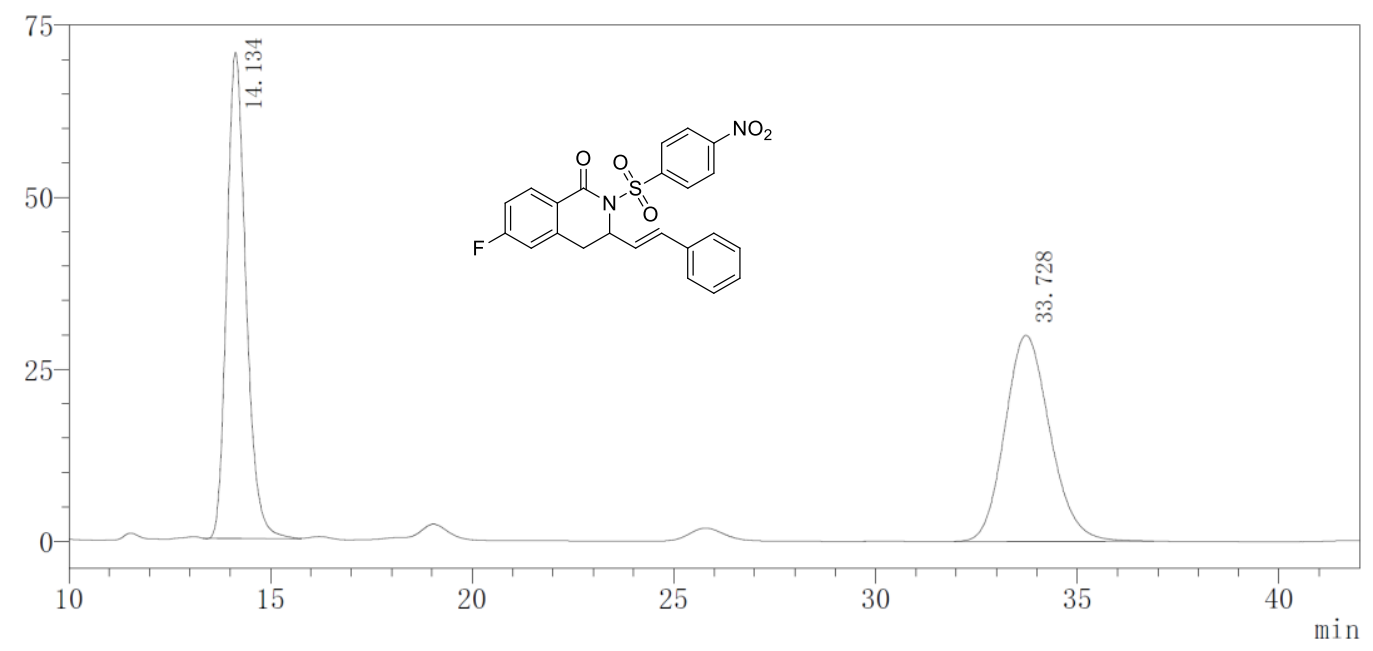

\begin{tabular}{|c|l|l|l|l|}
\hline $\begin{array}{c}\text { Peak } \\
\#\end{array}$ & $\begin{array}{c}\text { RetTime } \\
(\mathrm{min})\end{array}$ & $\begin{array}{c}\text { Area } \\
(\mathrm{mV} * \mathrm{~S})\end{array}$ & $\begin{array}{c}\text { Height } \\
(\mathrm{mV})\end{array}$ & $\begin{array}{c}\text { Area } \\
(\%)\end{array}$ \\
\hline 1 & 14.134 & 2344697 & 70633 & 50.229 \\
\hline 2 & 33.728 & 2323354 & 29926 & 49.771 \\
\hline
\end{tabular}

$\mathrm{mV}$

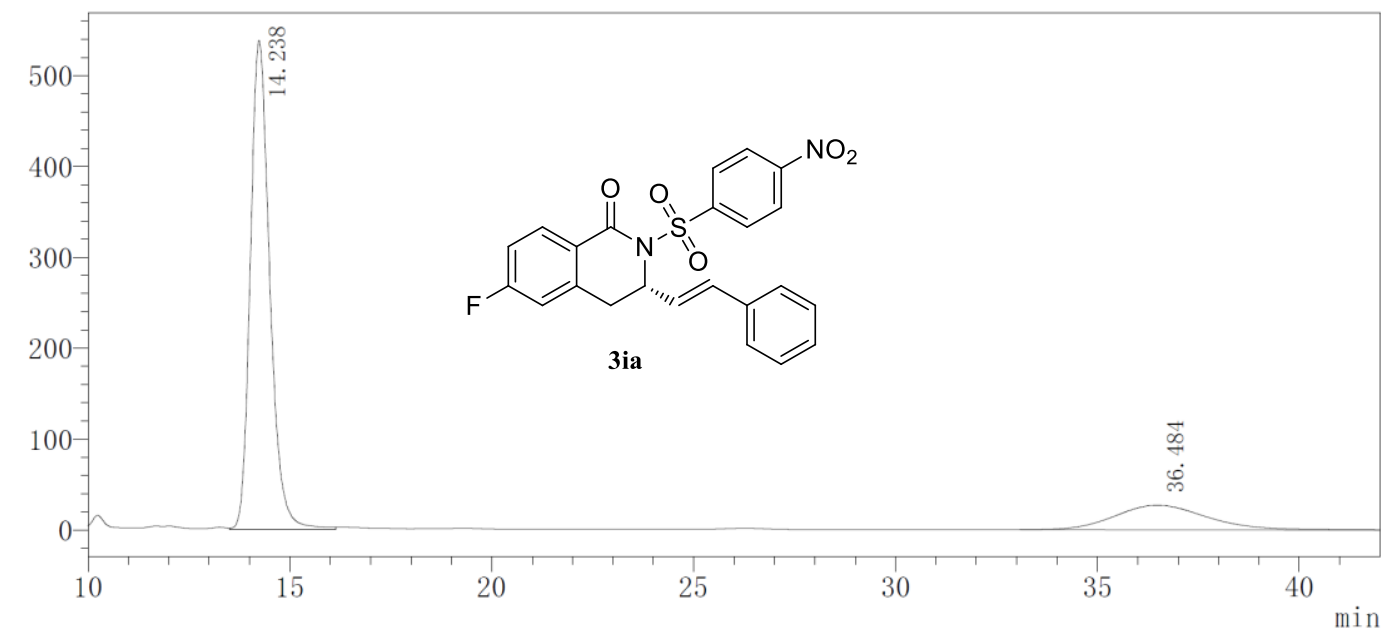

\begin{tabular}{|c|l|l|l|l|}
\hline $\begin{array}{c}\text { Peak } \\
\#\end{array}$ & $\begin{array}{c}\text { RetTime } \\
(\mathrm{min})\end{array}$ & $\begin{array}{c}\text { Area } \\
(\mathrm{mV} * \mathrm{~S})\end{array}$ & $\begin{array}{c}\text { Height } \\
(\mathrm{mV})\end{array}$ & $\begin{array}{c}\text { Area } \\
(\%)\end{array}$ \\
\hline 1 & 14.238 & 18062255 & 538280 & 80.702 \\
\hline 2 & 36.484 & 4319052 & 26954 & 19.298 \\
\hline
\end{tabular}




\section{$3 \mathbf{j a}$}

mV

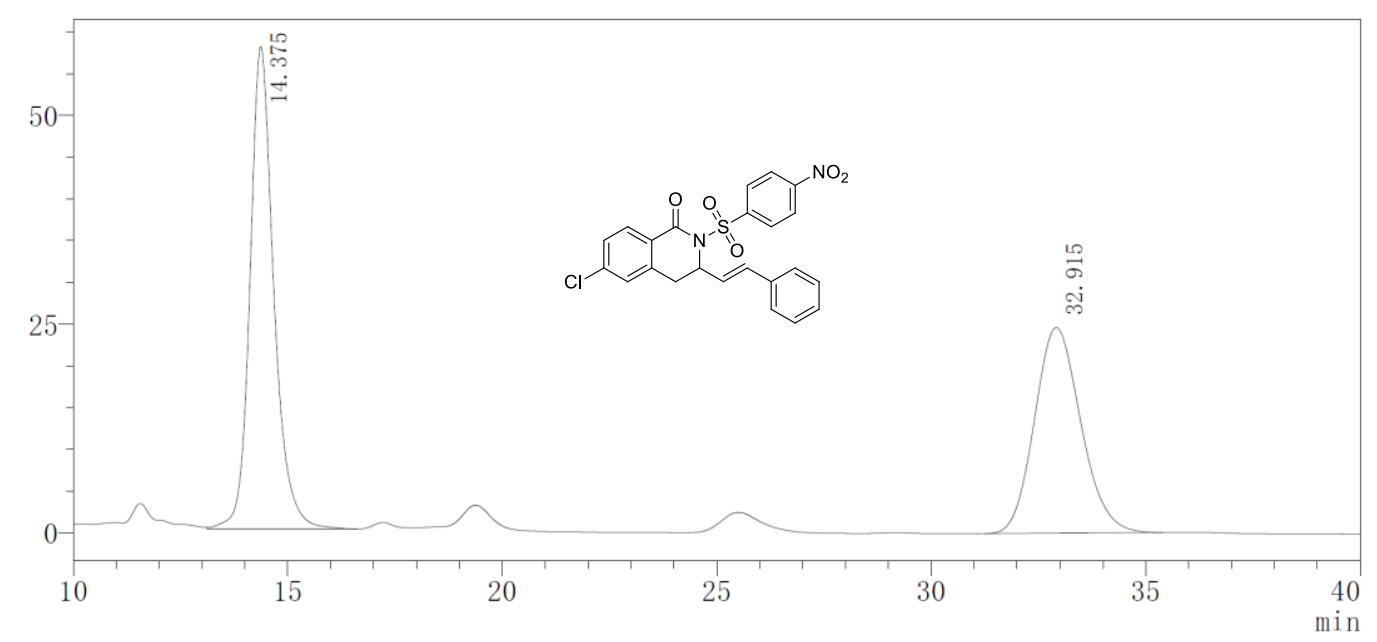

\begin{tabular}{|c|c|c|c|c|}
\hline $\begin{array}{c}\text { Peak } \\
\#\end{array}$ & $\begin{array}{c}\text { RetTime } \\
(\mathrm{min})\end{array}$ & $\begin{array}{c}\text { Area } \\
(\mathrm{mV} * \mathrm{~S})\end{array}$ & $\begin{array}{c}\text { Height } \\
(\mathrm{mV})\end{array}$ & $\begin{array}{c}\text { Area } \\
(\%)\end{array}$ \\
\hline 1 & 14.375 & 2239470 & 57818 & 54.717 \\
\hline 2 & 32.915 & 1853347 & 24659 & 45.283 \\
\hline
\end{tabular}

$\mathrm{mV}$

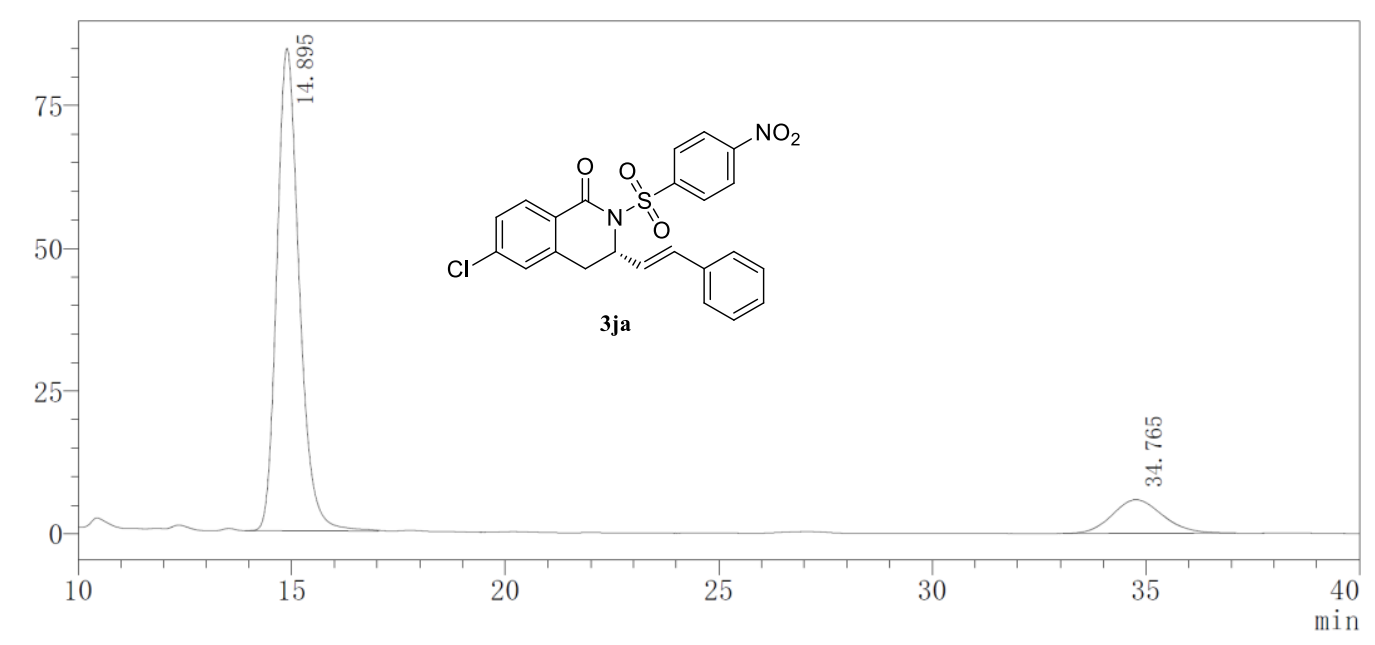

\begin{tabular}{|c|c|c|c|c|}
\hline $\begin{array}{c}\text { Peak } \\
\#\end{array}$ & $\begin{array}{c}\text { RetTime } \\
(\mathrm{min})\end{array}$ & $\begin{array}{c}\text { Area } \\
(\mathrm{mV} * \mathrm{~S})\end{array}$ & $\begin{array}{c}\text { Height } \\
(\mathrm{mV})\end{array}$ & $\begin{array}{c}\text { Area } \\
(\%)\end{array}$ \\
\hline 1 & 14.895 & 3093874 & 84558 & 86.559 \\
\hline 2 & 34.765 & 480403 & 5869 & 13.441 \\
\hline
\end{tabular}


3ka

$\mathrm{mV}$

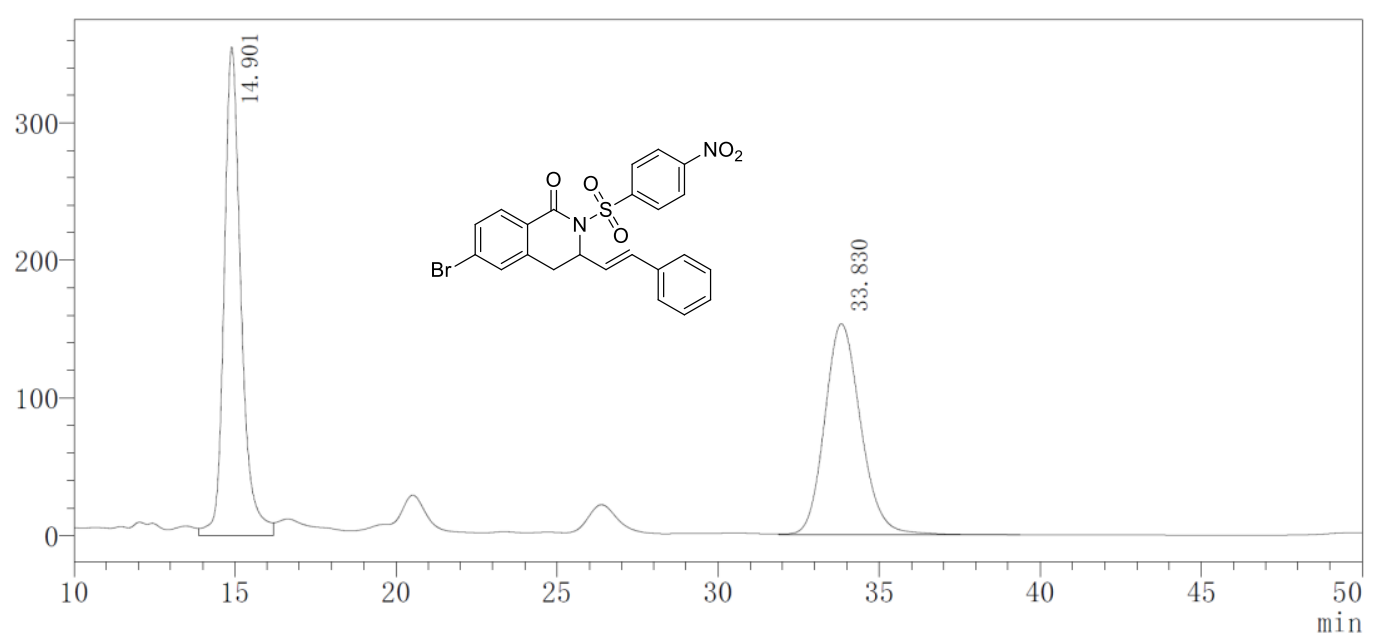

\begin{tabular}{|c|c|c|c|c|}
\hline $\begin{array}{c}\text { Peak } \\
\#\end{array}$ & $\begin{array}{c}\text { RetTime } \\
(\mathrm{min})\end{array}$ & $\begin{array}{c}\text { Area } \\
(\mathrm{mV} * \mathrm{~S})\end{array}$ & $\begin{array}{c}\text { Height } \\
(\mathrm{mV})\end{array}$ & $\begin{array}{c}\text { Area } \\
(\%)\end{array}$ \\
\hline 1 & 14.901 & 12984958 & 354765 & 51.945 \\
\hline 2 & 33.830 & 12012434 & 153527 & 48.055 \\
\hline
\end{tabular}

$\mathrm{mV}$

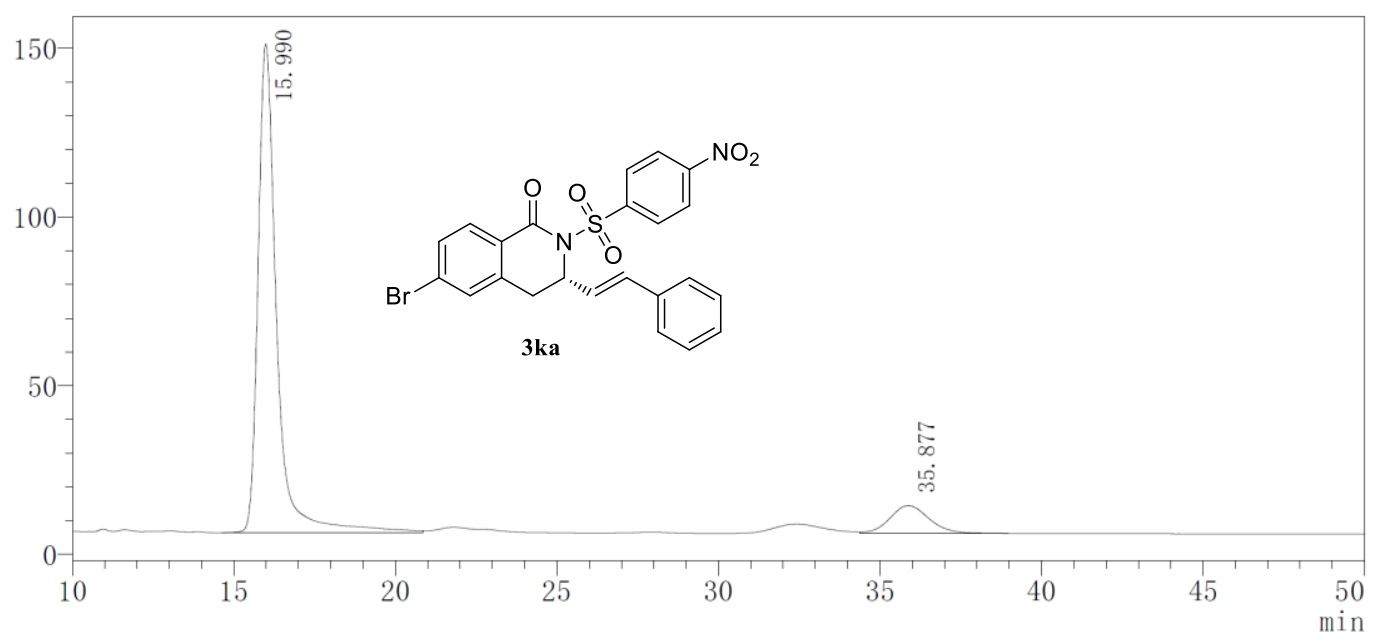

\begin{tabular}{|c|c|c|c|c|}
\hline $\begin{array}{c}\text { Peak } \\
\#\end{array}$ & $\begin{array}{c}\text { RetTime } \\
(\mathrm{min})\end{array}$ & $\begin{array}{c}\text { Area } \\
(\mathrm{mV} * \mathrm{~S})\end{array}$ & $\begin{array}{c}\text { Height } \\
(\mathrm{mV})\end{array}$ & $\begin{array}{c}\text { Area } \\
(\%)\end{array}$ \\
\hline 1 & 15.990 & 5833351 & 144781 & 89.223 \\
\hline 2 & 35.877 & 704597 & 8254 & 10.777 \\
\hline
\end{tabular}


$31 a$

$\mathrm{mV}$

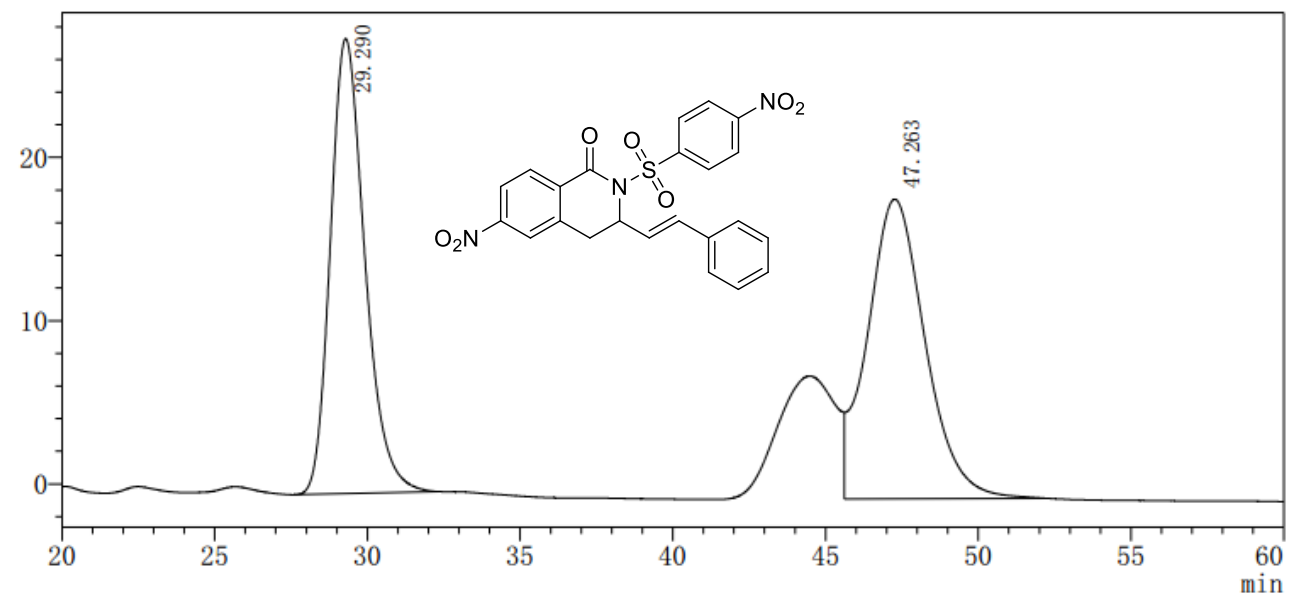

\begin{tabular}{|l|l|l|l|l|}
\hline $\begin{array}{l}\text { Peak } \\
\#\end{array}$ & $\begin{array}{l}\text { RetTime } \\
(\mathrm{min})\end{array}$ & $\begin{array}{l}\text { Area } \\
(\mathrm{mV} * \mathrm{~S})\end{array}$ & $\begin{array}{l}\text { Height } \\
(\mathrm{mV})\end{array}$ & $\begin{array}{l}\text { Area } \\
(\%)\end{array}$ \\
\hline 1 & 29.290 & 2190423 & 27873 & 47.524 \\
\hline 2 & 47.263 & 2418697 & 18347 & 52.476 \\
\hline
\end{tabular}

$\mathrm{mV}$

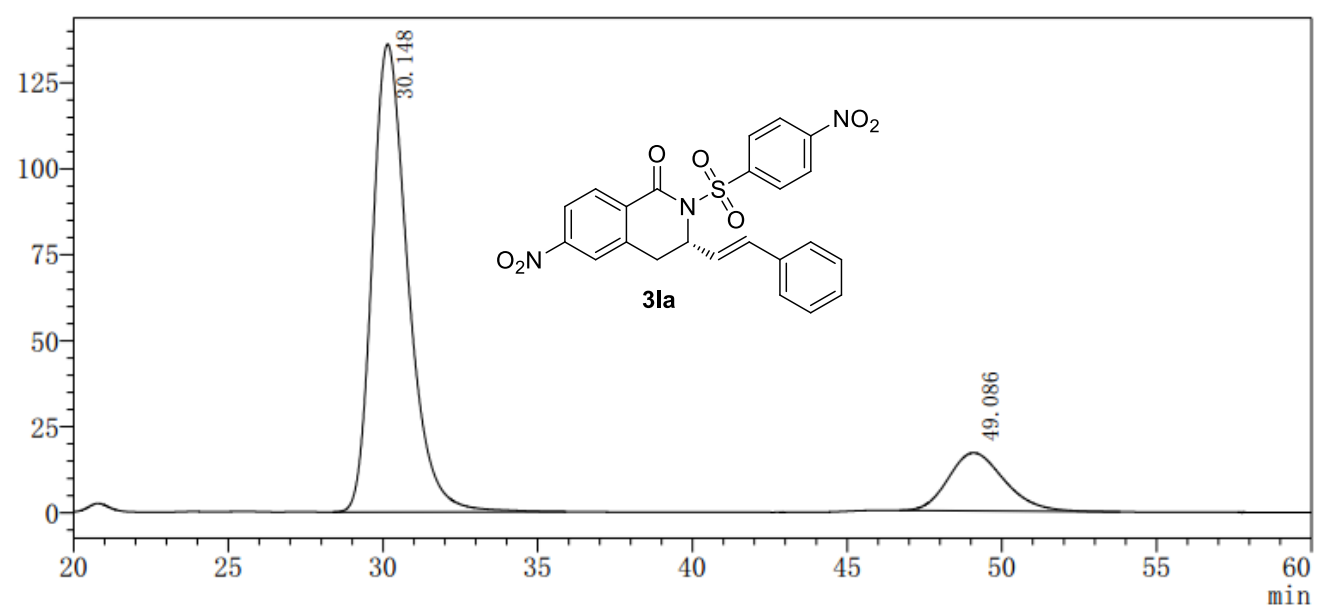

\begin{tabular}{|l|l|l|l|l|}
\hline $\begin{array}{l}\text { Peak } \\
\#\end{array}$ & $\begin{array}{l}\text { RetTime } \\
(\mathrm{min})\end{array}$ & $\begin{array}{l}\text { Area } \\
(\mathrm{mV} * \mathrm{~s})\end{array}$ & $\begin{array}{l}\text { Height } \\
(\mathrm{mV})\end{array}$ & $\begin{array}{l}\text { Area } \\
(\%)\end{array}$ \\
\hline 1 & 30.148 & 10886878 & 136061 & 83.650 \\
\hline 2 & 49.086 & 2127929 & 16872 & 16.350 \\
\hline
\end{tabular}


3ma

$\mathrm{mV}$

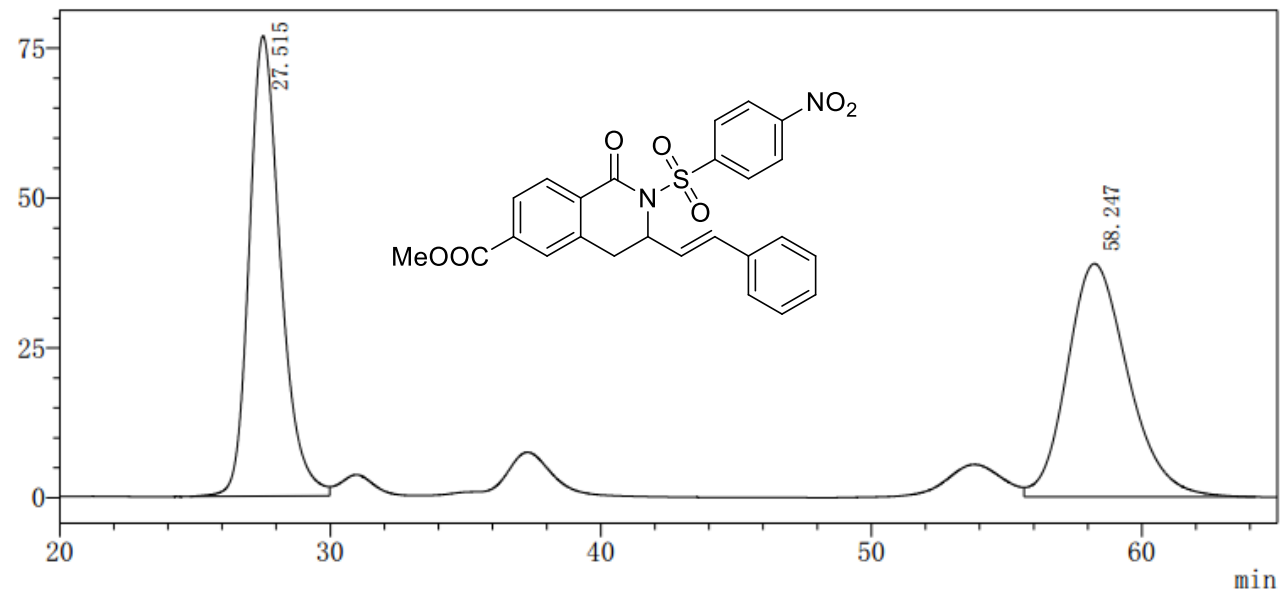

\begin{tabular}{|l|l|l|l|l|}
\hline $\begin{array}{l}\text { Peak } \\
\#\end{array}$ & $\begin{array}{l}\text { RetTime } \\
(\mathrm{min})\end{array}$ & $\begin{array}{l}\text { Area } \\
(\mathrm{mV} * \mathrm{~S})\end{array}$ & $\begin{array}{l}\text { Height } \\
(\mathrm{mV})\end{array}$ & $\begin{array}{l}\text { Area } \\
(\%)\end{array}$ \\
\hline 1 & 27.515 & 6186464 & 76753 & 50.403 \\
\hline 2 & 58.247 & 6087642 & 38888 & 49.597 \\
\hline
\end{tabular}

$\mathrm{mV}$

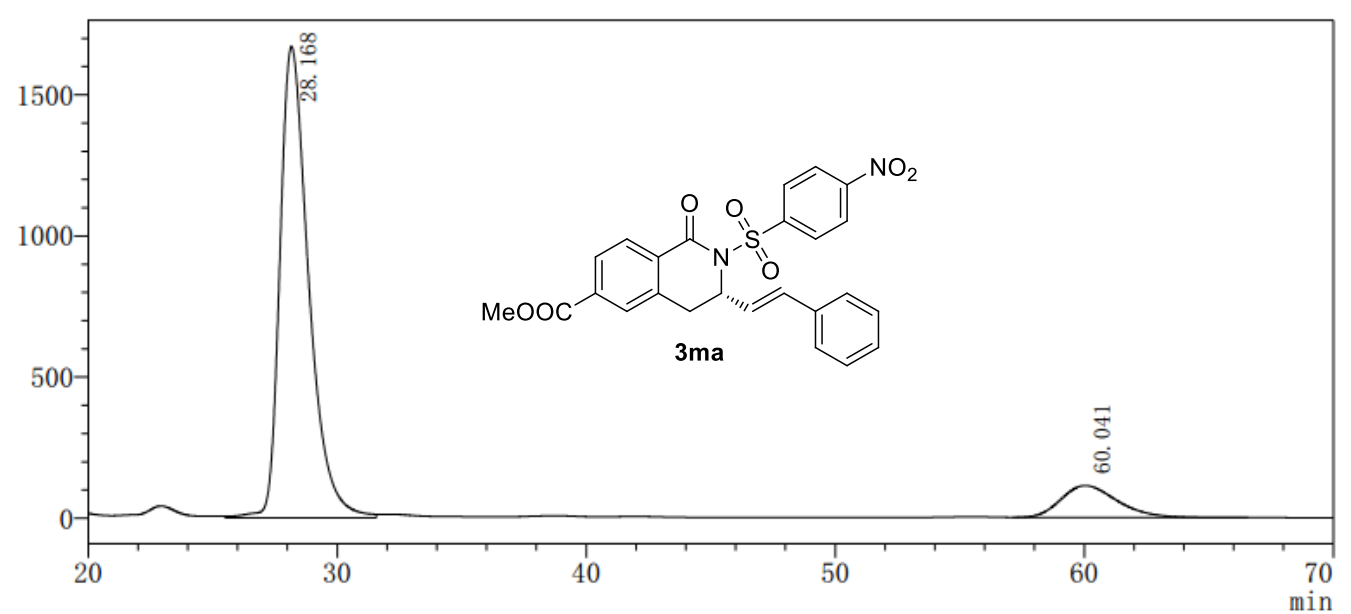

\begin{tabular}{|l|l|l|l|l|}
\hline $\begin{array}{l}\text { Peak } \\
\#\end{array}$ & $\begin{array}{l}\text { RetTime } \\
(\mathrm{min})\end{array}$ & $\begin{array}{l}\text { Area } \\
(\mathrm{mV} * \mathrm{~S})\end{array}$ & $\begin{array}{l}\text { Height } \\
(\mathrm{mV})\end{array}$ & $\begin{array}{l}\text { Area } \\
(\%)\end{array}$ \\
\hline 1 & 28.168 & 134297070 & 1669542 & 88.197 \\
\hline 2 & 60.041 & 17973052 & 113411 & 11.803 \\
\hline
\end{tabular}


$\mathrm{mV}$

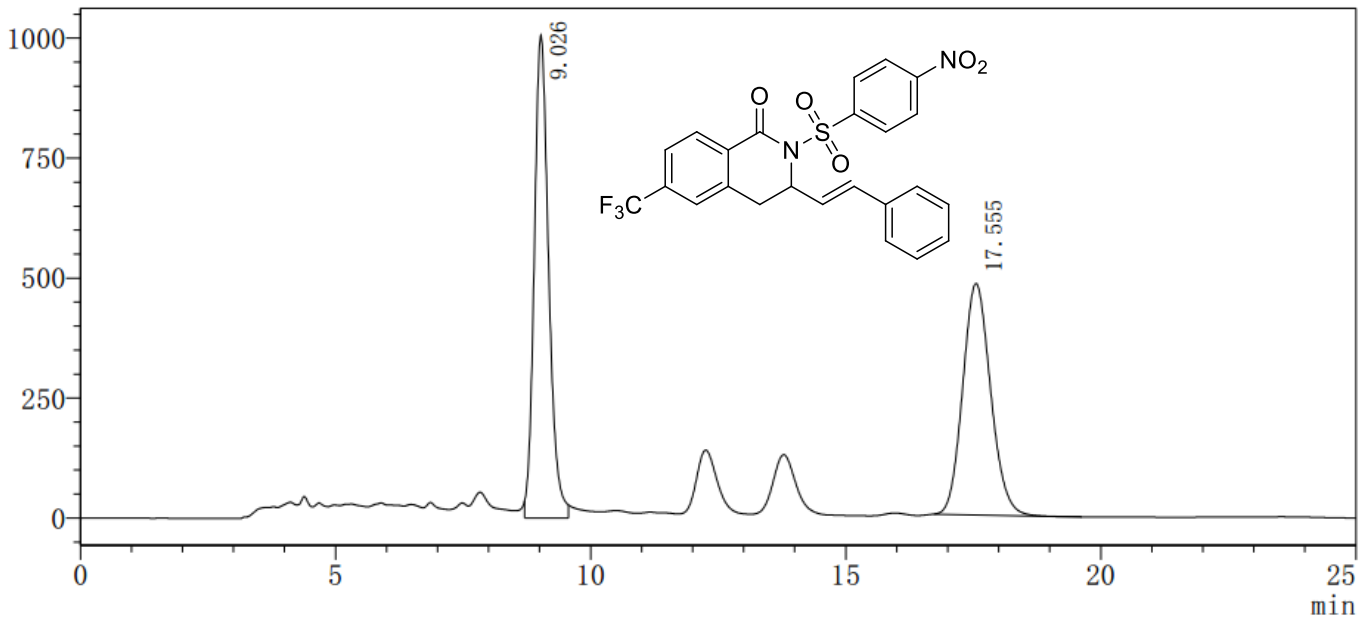

\begin{tabular}{|l|l|l|l|l|}
\hline $\begin{array}{l}\text { Peak } \\
\#\end{array}$ & $\begin{array}{l}\text { RetTime } \\
(\mathrm{min})\end{array}$ & $\begin{array}{l}\text { Area } \\
(\mathrm{mV} * \mathrm{~S})\end{array}$ & $\begin{array}{l}\text { Height } \\
(\mathrm{mV})\end{array}$ & $\begin{array}{l}\text { Area } \\
(\%)\end{array}$ \\
\hline 1 & 9.026 & 19729581 & 1005888 & 51.783 \\
\hline 2 & 17.555 & 18371249 & 482247 & 48.217 \\
\hline
\end{tabular}

$\mathrm{mV}$

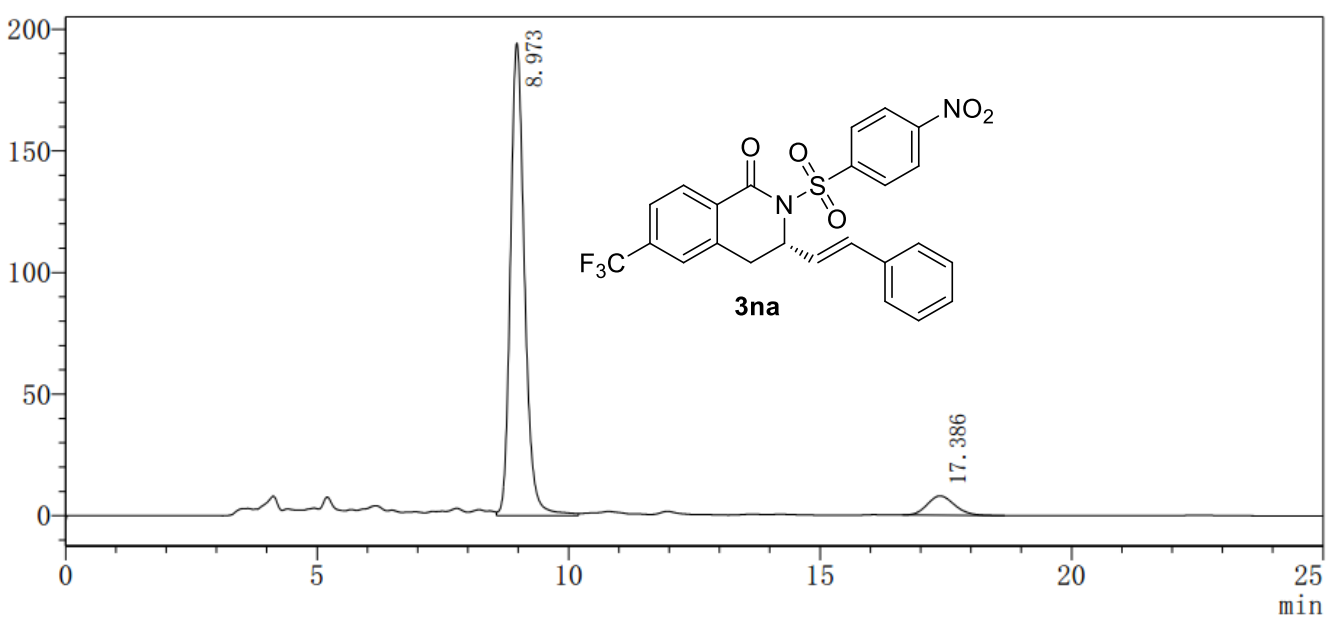

\begin{tabular}{|l|l|l|l|l|}
\hline $\begin{array}{l}\text { Peak } \\
\#\end{array}$ & $\begin{array}{l}\text { RetTime } \\
(\mathrm{min})\end{array}$ & $\begin{array}{l}\text { Area } \\
(\mathrm{mV} * \mathrm{~S})\end{array}$ & $\begin{array}{l}\text { Height } \\
(\mathrm{mV})\end{array}$ & $\begin{array}{l}\text { Area } \\
(\%)\end{array}$ \\
\hline 1 & 8.973 & 3783077 & 194119 & 92.667 \\
\hline 2 & 17.386 & 299377 & 7935 & 7.333 \\
\hline
\end{tabular}


3oa

$\mathrm{mV}$

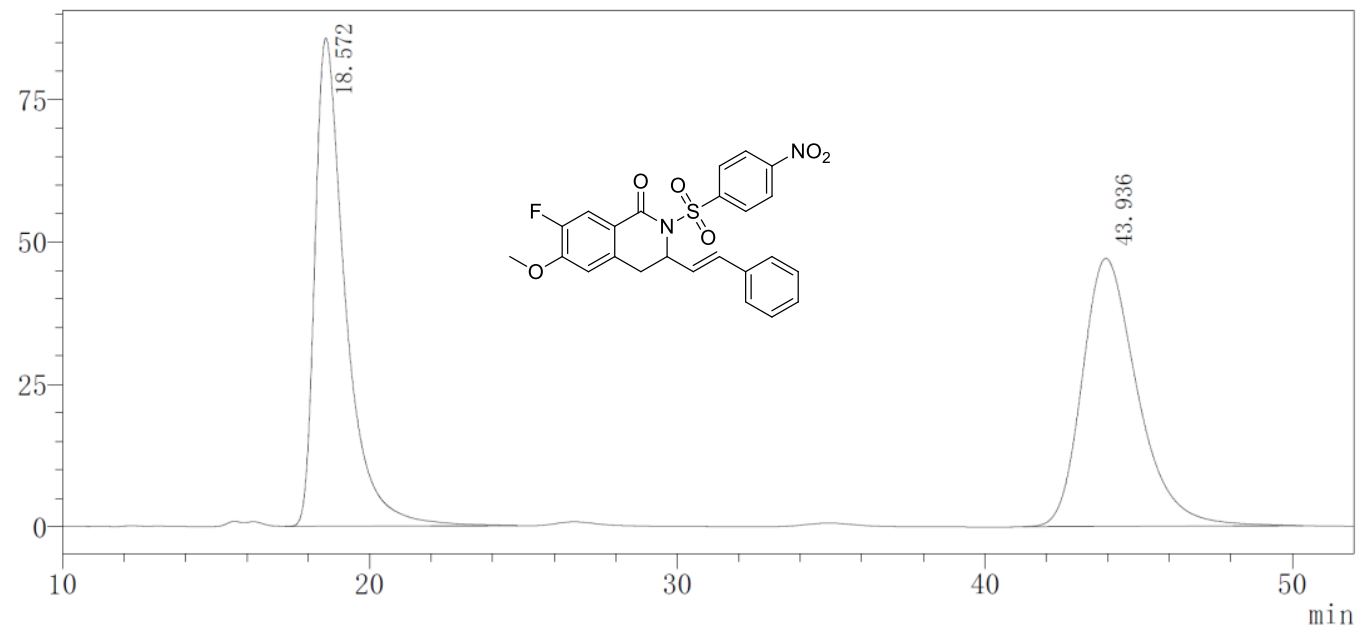

\begin{tabular}{|c|c|c|l|c|}
\hline $\begin{array}{c}\text { Peak } \\
\#\end{array}$ & $\begin{array}{c}\text { RetTime } \\
(\mathrm{min})\end{array}$ & $\begin{array}{c}\text { Area } \\
(\mathrm{mV} * \mathrm{~S})\end{array}$ & $\begin{array}{c}\text { Height } \\
(\mathrm{mV})\end{array}$ & $\begin{array}{c}\text { Area } \\
(\%)\end{array}$ \\
\hline 1 & 18.572 & 5919600 & 85686 & 50.181 \\
\hline 2 & 43.936 & 5876861 & 47045 & 49.819 \\
\hline
\end{tabular}

$\mathrm{mV}$

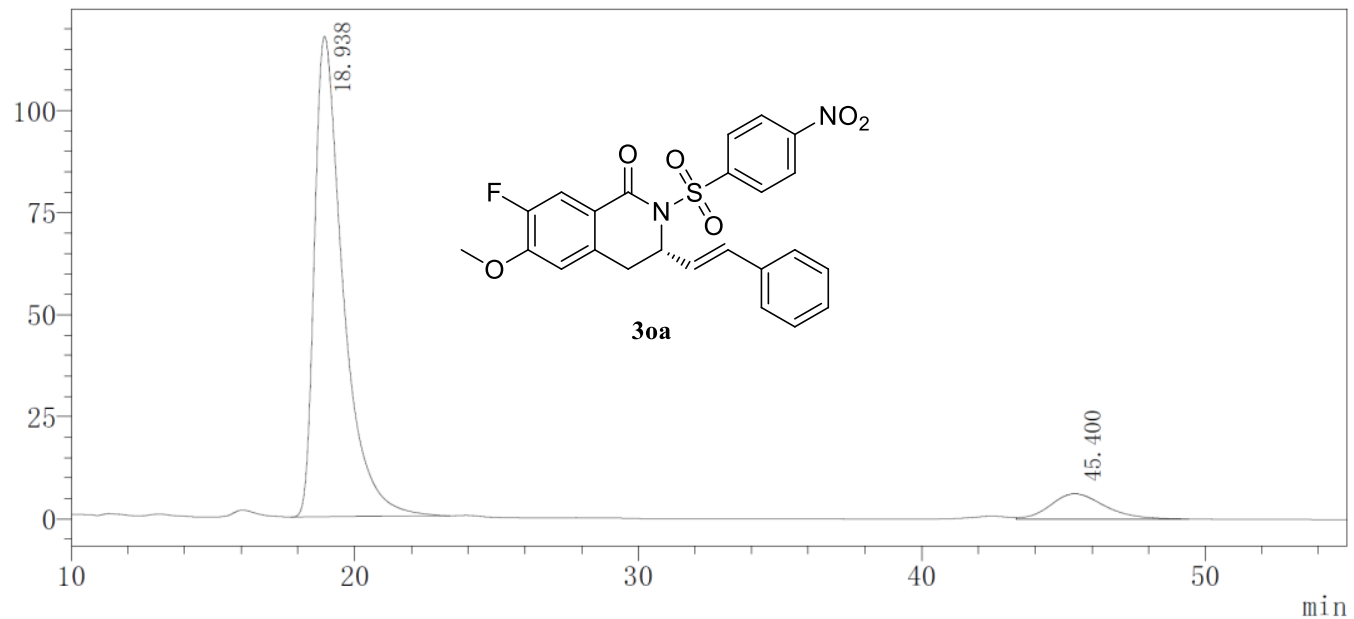

\begin{tabular}{|c|l|l|l|l|}
\hline $\begin{array}{c}\text { Peak } \\
\#\end{array}$ & $\begin{array}{c}\text { RetTime } \\
(\mathrm{min})\end{array}$ & $\begin{array}{c}\text { Area } \\
(\mathrm{mV} * \mathrm{~S})\end{array}$ & $\begin{array}{c}\text { Height } \\
(\mathrm{mV})\end{array}$ & $\begin{array}{c}\text { Area } \\
(\%)\end{array}$ \\
\hline 1 & 18.938 & 8245488 & 117619 & 91.019 \\
\hline 2 & 45.400 & 813641 & 6149 & 8.981 \\
\hline
\end{tabular}


$3 a b$

$\mathrm{mV}$

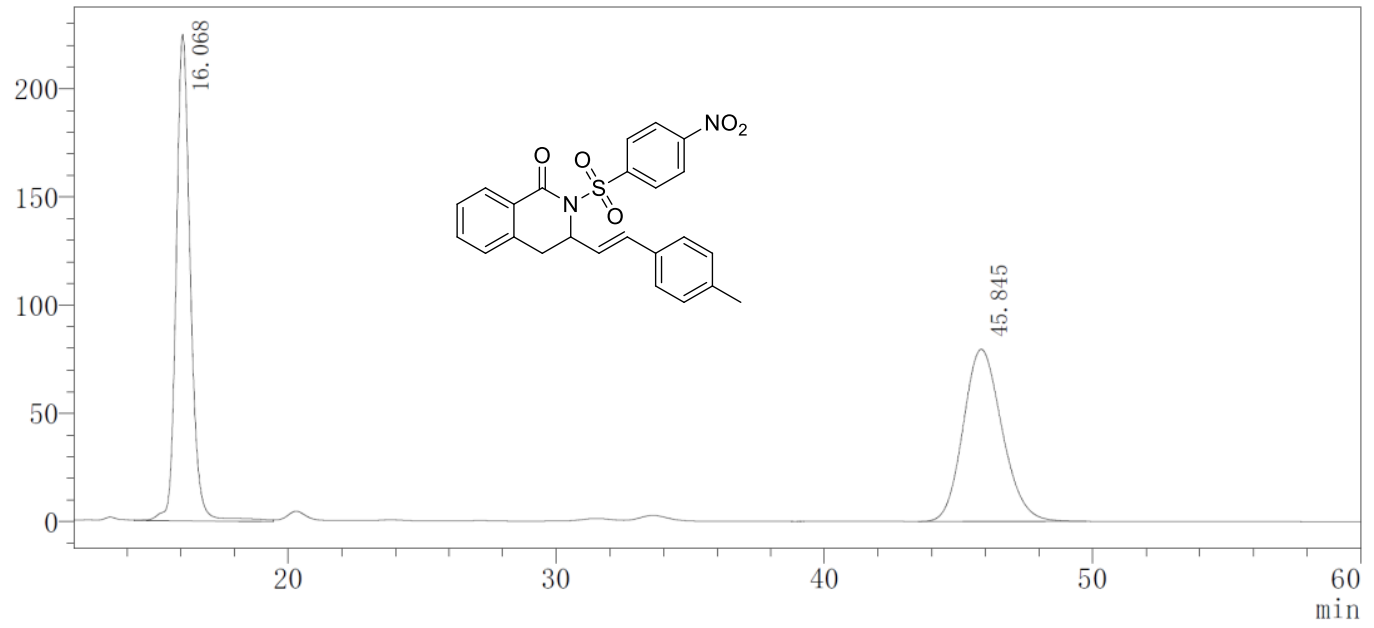

\begin{tabular}{|c|c|c|c|c|}
\hline $\begin{array}{c}\text { Peak } \\
\#\end{array}$ & $\begin{array}{c}\text { RetTime } \\
(\mathrm{min})\end{array}$ & $\begin{array}{c}\text { Area } \\
(\mathrm{mV} * \mathrm{~S})\end{array}$ & $\begin{array}{c}\text { Height } \\
(\mathrm{mV})\end{array}$ & $\begin{array}{c}\text { Area } \\
(\%)\end{array}$ \\
\hline 1 & 16.068 & 8331785 & 224619 & 50.979 \\
\hline 2 & 45.845 & 8011634 & 79541 & 49.021 \\
\hline
\end{tabular}

$\mathrm{mV}$

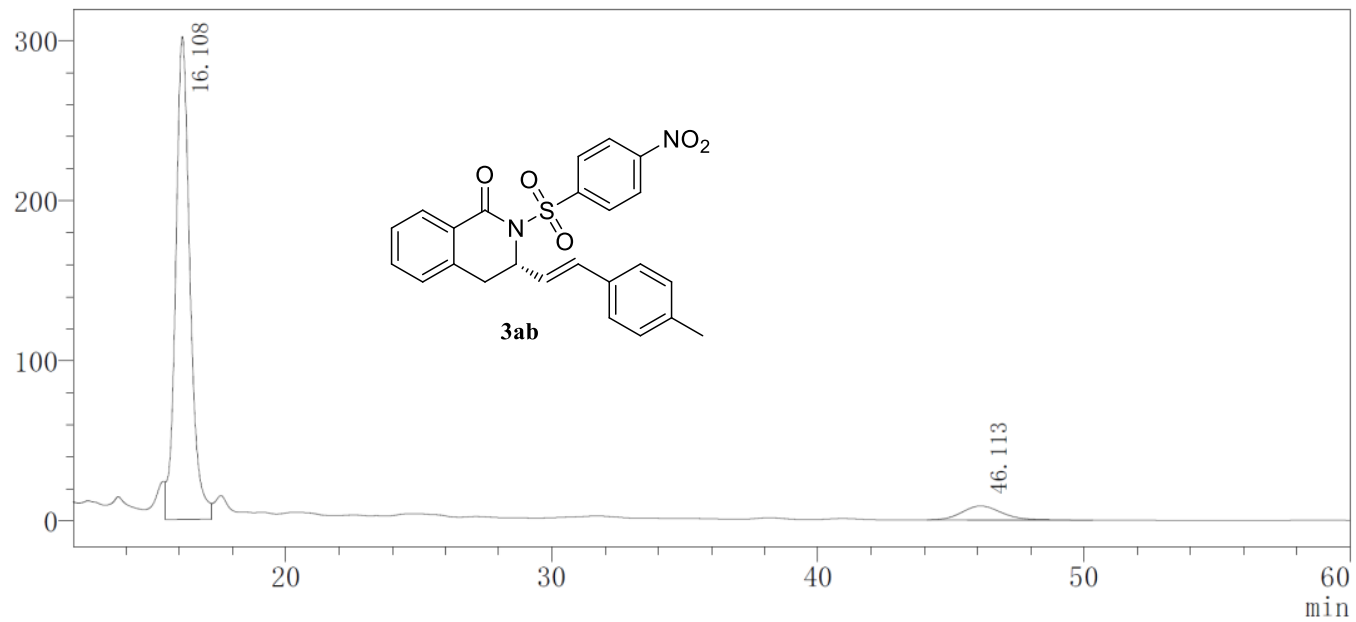

\begin{tabular}{|c|l|l|l|l|}
\hline $\begin{array}{c}\text { Peak } \\
\#\end{array}$ & $\begin{array}{c}\text { RetTime } \\
(\mathrm{min})\end{array}$ & $\begin{array}{c}\text { Area } \\
(\mathrm{mV} * \mathrm{~S})\end{array}$ & $\begin{array}{c}\text { Height } \\
(\mathrm{mV})\end{array}$ & $\begin{array}{c}\text { Area } \\
(\%)\end{array}$ \\
\hline 1 & 16.108 & 11326977 & 301510 & 92.565 \\
\hline 2 & 46.113 & 909807 & 8768 & 7.435 \\
\hline
\end{tabular}


$3 a c$

$\mathrm{mV}$

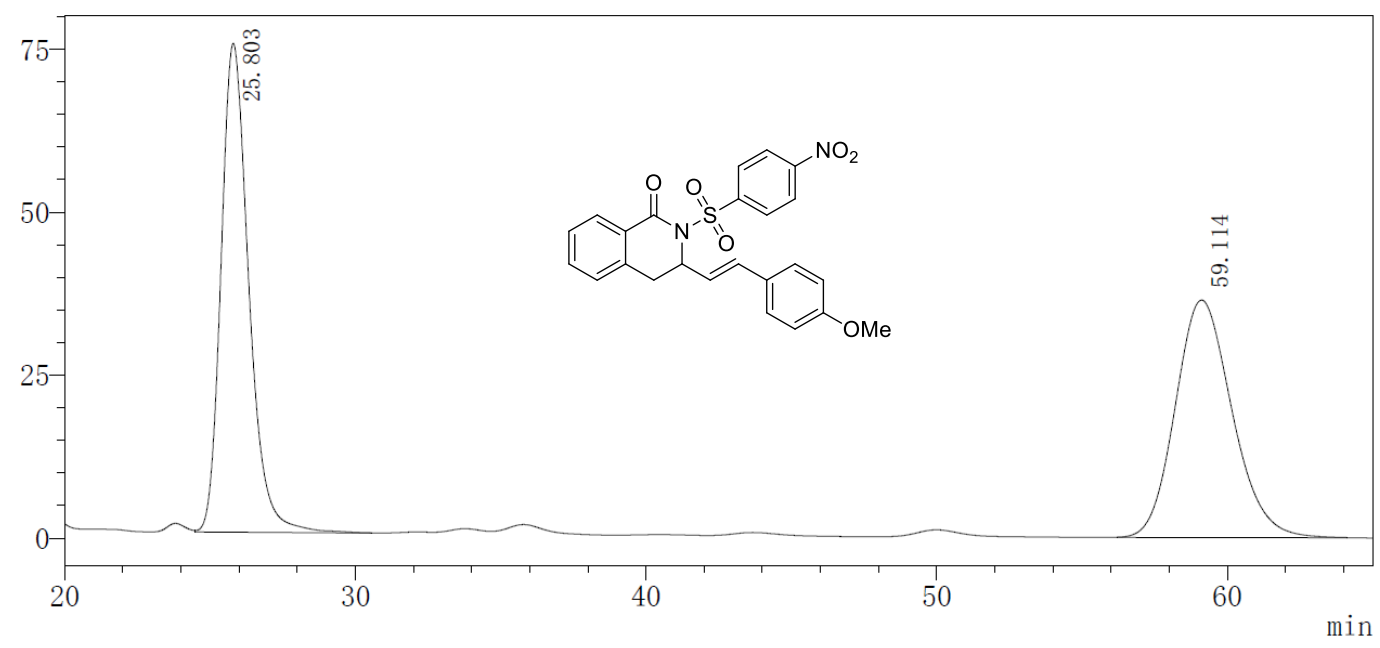

\begin{tabular}{|c|l|l|l|l|}
\hline $\begin{array}{c}\text { Peak } \\
\#\end{array}$ & $\begin{array}{c}\text { RetTime } \\
(\mathrm{min})\end{array}$ & $\begin{array}{c}\text { Area } \\
(\mathrm{mV} * \mathrm{~S})\end{array}$ & $\begin{array}{c}\text { Height } \\
(\mathrm{mV})\end{array}$ & $\begin{array}{c}\text { Area } \\
(\%)\end{array}$ \\
\hline 1 & 25.803 & 4971115 & 75016 & 50.210 \\
\hline 2 & 59.114 & 4929530 & 36410 & 49.790 \\
\hline
\end{tabular}

$\mathrm{mV}$

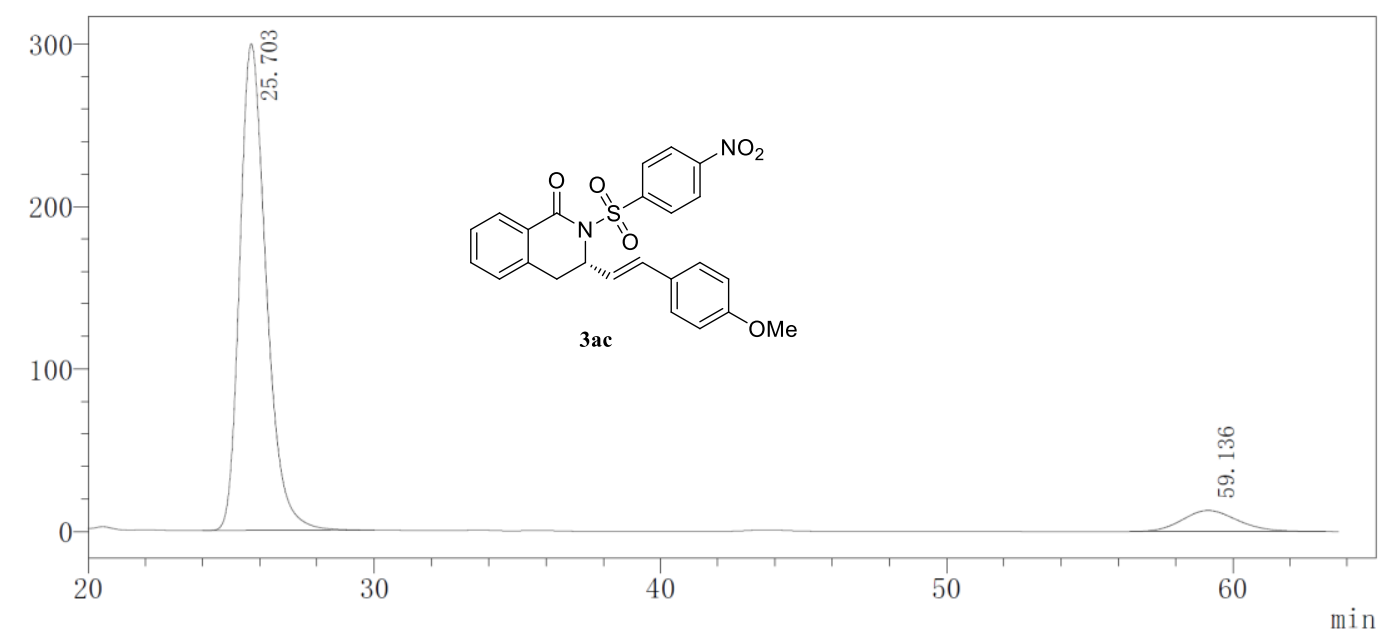

\begin{tabular}{|c|l|l|l|l|}
\hline $\begin{array}{c}\text { Peak } \\
\#\end{array}$ & $\begin{array}{c}\text { RetTime } \\
(\mathrm{min})\end{array}$ & $\begin{array}{c}\text { Area } \\
(\mathrm{mV} * \mathrm{~S})\end{array}$ & $\begin{array}{c}\text { Height } \\
(\mathrm{mV})\end{array}$ & $\begin{array}{c}\text { Area } \\
(\%)\end{array}$ \\
\hline 1 & 25.703 & 19251653 & 299580 & 91.721 \\
\hline 2 & 59.136 & 1737677 & 12854 & 8.279 \\
\hline
\end{tabular}


3ad

mV

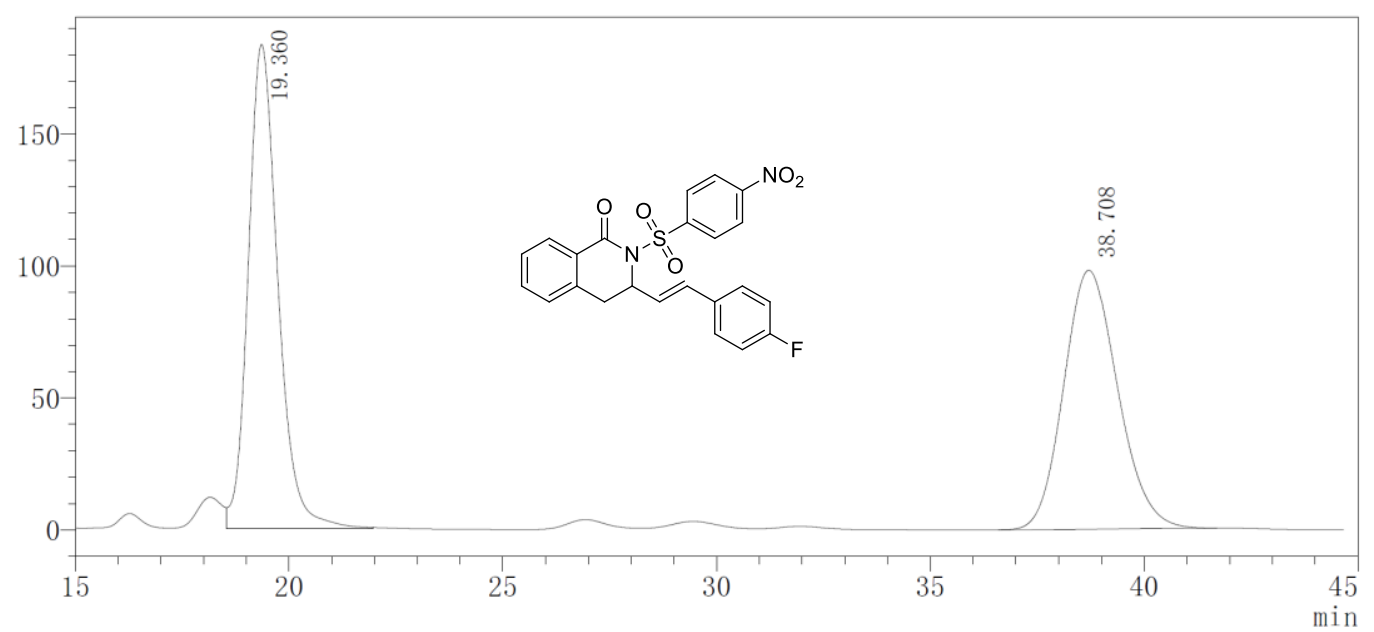

\begin{tabular}{|c|l|l|l|l|}
\hline $\begin{array}{c}\text { Peak } \\
\#\end{array}$ & $\begin{array}{c}\text { RetTime } \\
(\mathrm{min})\end{array}$ & $\begin{array}{c}\text { Area } \\
(\mathrm{mV} * \mathrm{~S})\end{array}$ & $\begin{array}{c}\text { Height } \\
(\mathrm{mV})\end{array}$ & $\begin{array}{c}\text { Area } \\
(\%)\end{array}$ \\
\hline 1 & 19.360 & 8919301 & 183669 & 50.925 \\
\hline 2 & 38.708 & 8595378 & 98093 & 49.075 \\
\hline $\mathrm{mV}$
\end{tabular}

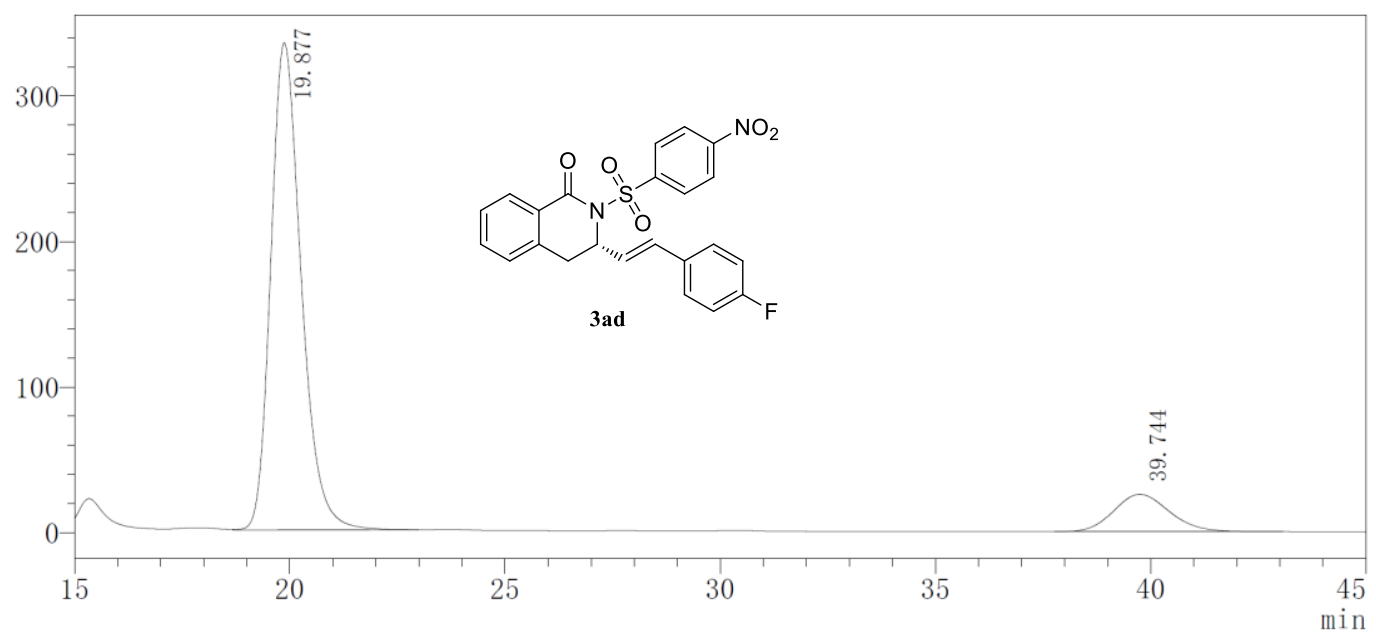

\begin{tabular}{|c|c|c|c|c|}
\hline $\begin{array}{c}\text { Peak } \\
\#\end{array}$ & $\begin{array}{c}\text { RetTime } \\
(\mathrm{min})\end{array}$ & $\begin{array}{c}\text { Area } \\
(\mathrm{mV} * \mathrm{~S})\end{array}$ & $\begin{array}{c}\text { Height } \\
(\mathrm{mV})\end{array}$ & $\begin{array}{c}\text { Area } \\
(\%)\end{array}$ \\
\hline 1 & 19.877 & 16496459 & 334948 & 87.680 \\
\hline 2 & 39.744 & 2317909 & 25690 & 12.320 \\
\hline
\end{tabular}


3 ae

mV

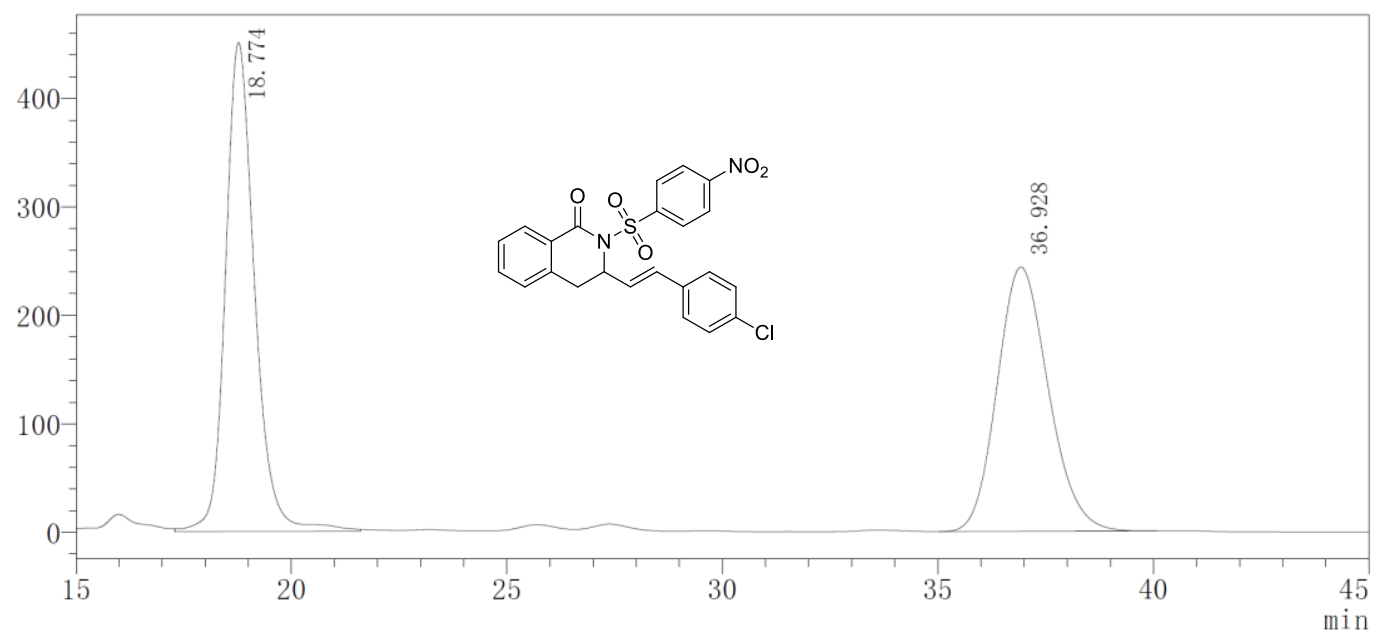

\begin{tabular}{|c|l|c|c|c|}
\hline $\begin{array}{c}\text { Peak } \\
\#\end{array}$ & $\begin{array}{c}\text { RetTime } \\
(\mathrm{min})\end{array}$ & $\begin{array}{c}\text { Area } \\
(\mathrm{mV} * \mathrm{~S})\end{array}$ & $\begin{array}{c}\text { Height } \\
(\mathrm{mV})\end{array}$ & $\begin{array}{c}\text { Area } \\
(\%)\end{array}$ \\
\hline 1 & 18.774 & 21271837 & 450471 & 51.321 \\
\hline 2 & 36.928 & 20176605 & 243537 & 48.679 \\
\hline
\end{tabular}

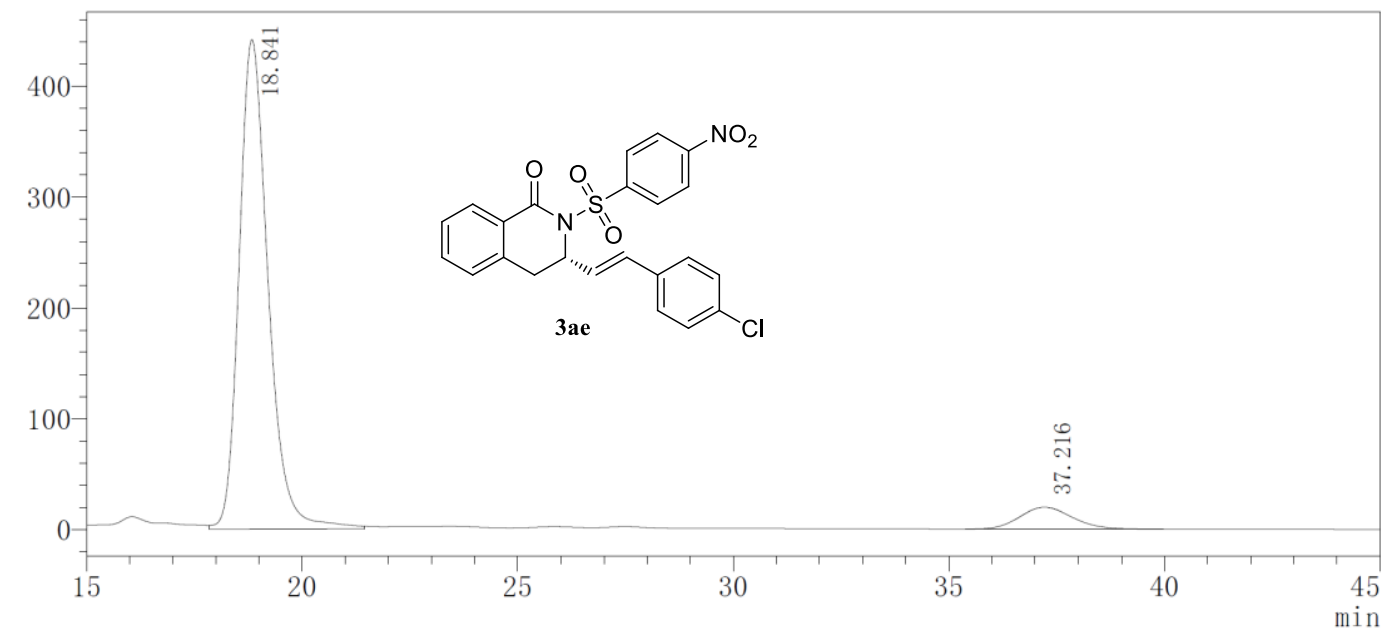

\begin{tabular}{|c|l|l|l|l|}
\hline $\begin{array}{c}\text { Peak } \\
\#\end{array}$ & $\begin{array}{c}\text { RetTime } \\
(\mathrm{min})\end{array}$ & $\begin{array}{c}\text { Area } \\
(\mathrm{mV} * \mathrm{~S})\end{array}$ & $\begin{array}{c}\text { Height } \\
(\mathrm{mV})\end{array}$ & $\begin{array}{c}\text { Area } \\
(\%)\end{array}$ \\
\hline 1 & 18.841 & 20673879 & 441473 & 92.489 \\
\hline 2 & 37.216 & 1678967 & 19769 & 7.511 \\
\hline
\end{tabular}


$3 a f$

$\mathrm{mV}$

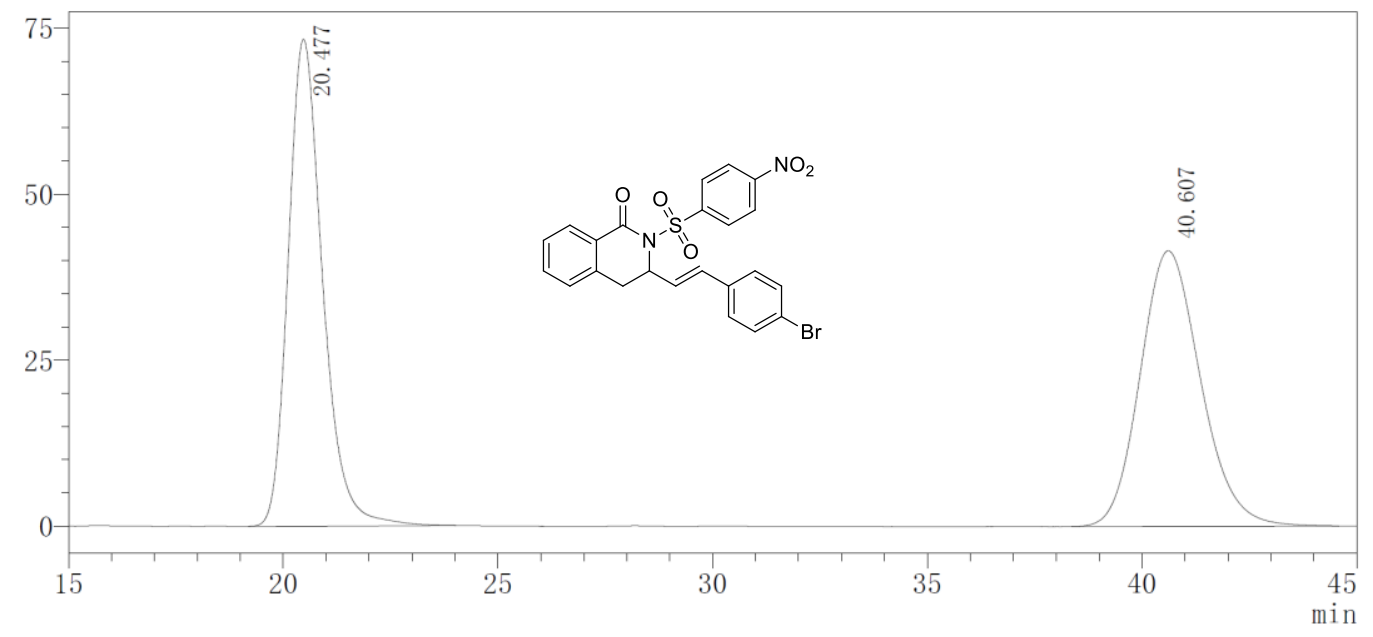

\begin{tabular}{|c|l|l|l|l|}
\hline $\begin{array}{c}\text { Peak } \\
\#\end{array}$ & $\begin{array}{c}\text { RetTime } \\
(\mathrm{min})\end{array}$ & $\begin{array}{c}\text { Area } \\
(\mathrm{mV} * \mathrm{~S})\end{array}$ & $\begin{array}{c}\text { Height } \\
(\mathrm{mV})\end{array}$ & $\begin{array}{c}\text { Area } \\
(\%)\end{array}$ \\
\hline 1 & 20.477 & 4076298 & 73294 & 50.108 \\
\hline 2 & 40.607 & 4058740 & 41458 & 49.892 \\
\hline
\end{tabular}

$\mathrm{mV}$

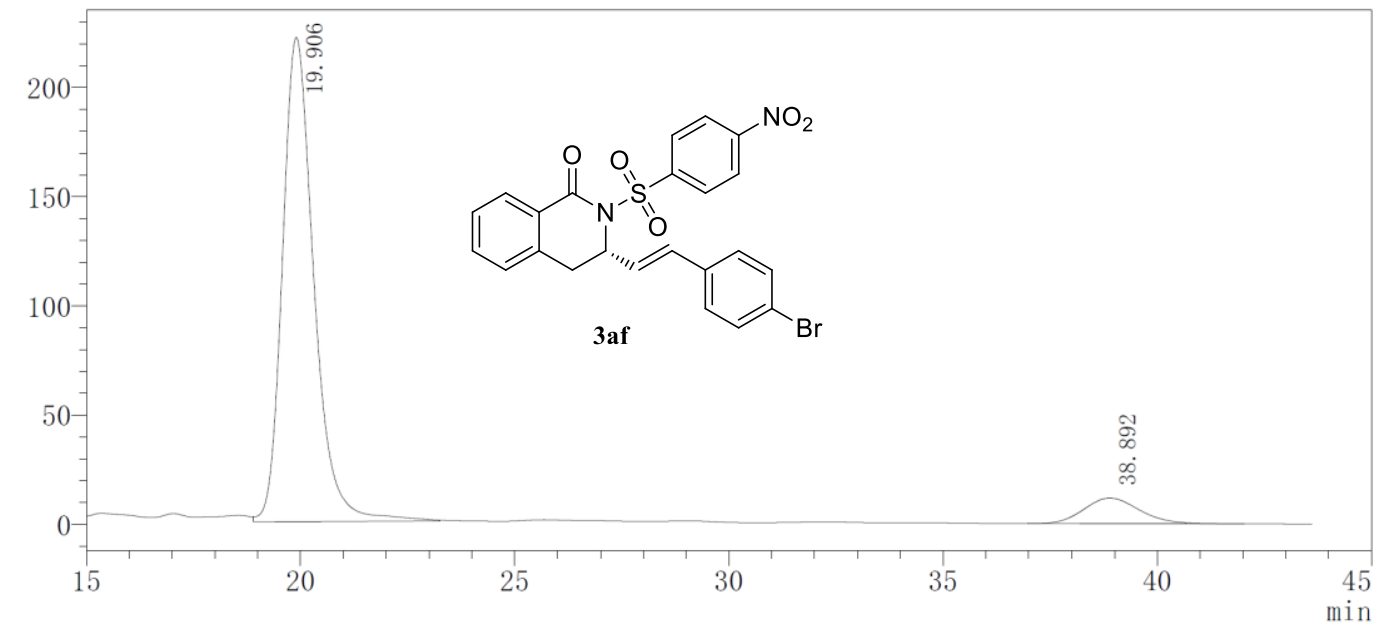

\begin{tabular}{|c|l|l|l|l|}
\hline $\begin{array}{c}\text { Peak } \\
\#\end{array}$ & $\begin{array}{c}\text { RetTime } \\
(\mathrm{min})\end{array}$ & $\begin{array}{c}\text { Area } \\
(\mathrm{mV} * \mathrm{~S})\end{array}$ & $\begin{array}{c}\text { Height } \\
(\mathrm{mV})\end{array}$ & $\begin{array}{c}\text { Area } \\
(\%)\end{array}$ \\
\hline 1 & 19.906 & 11343738 & 221746 & 91.441 \\
\hline 2 & 38.892 & 1061839 & 11709 & 8.559 \\
\hline
\end{tabular}


$3 a g$

$\mathrm{mV}$

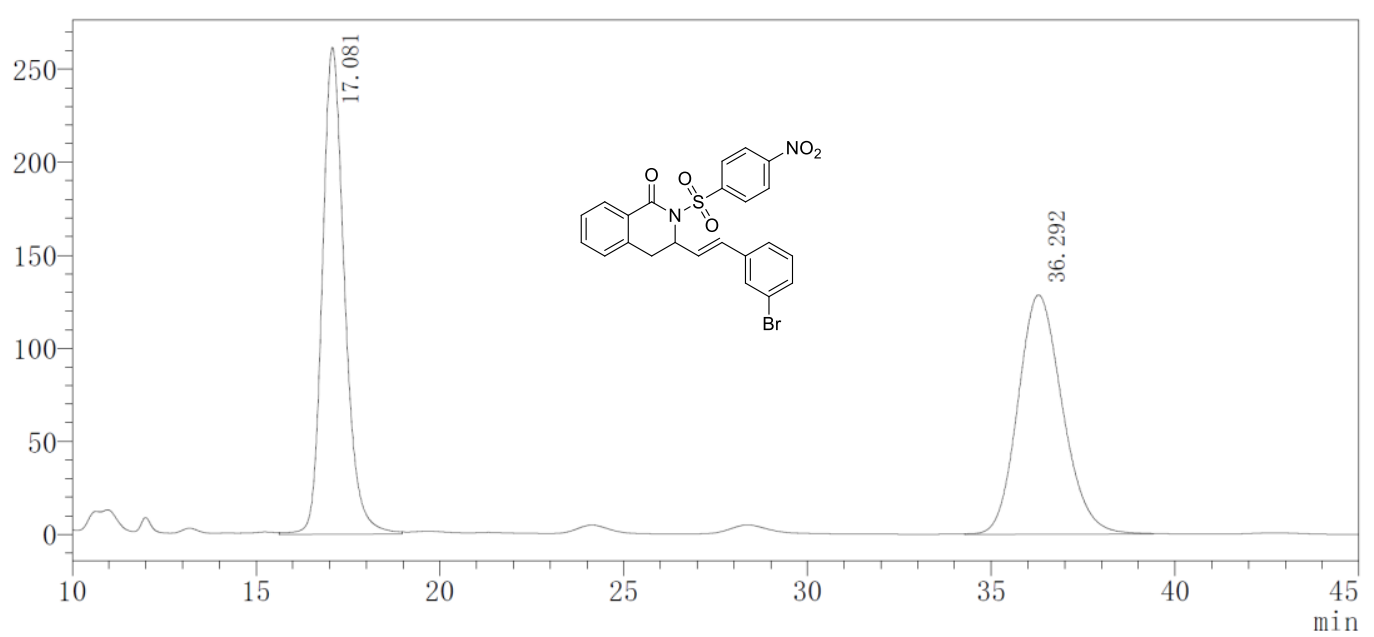

\begin{tabular}{|c|l|c|c|c|}
\hline $\begin{array}{c}\text { Peak } \\
\#\end{array}$ & $\begin{array}{c}\text { RetTime } \\
(\mathrm{min})\end{array}$ & $\begin{array}{c}\text { Area } \\
(\mathrm{mV} * \mathrm{~S})\end{array}$ & $\begin{array}{c}\text { Height } \\
(\mathrm{mV})\end{array}$ & $\begin{array}{c}\text { Area } \\
(\%)\end{array}$ \\
\hline 1 & 17.081 & 10999479 & 261544 & 50.408 \\
\hline 2 & 36.292 & 10821370 & 128583 & 49.592 \\
\hline $\mathrm{mV}$
\end{tabular}

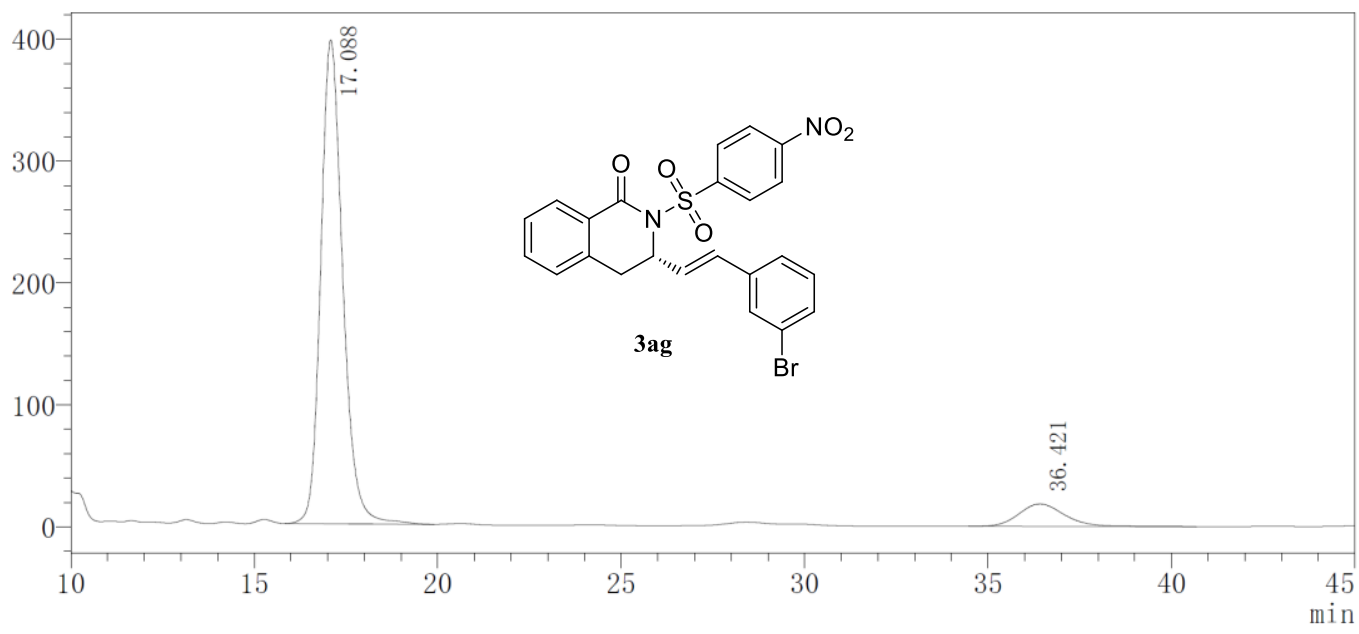

\begin{tabular}{|c|c|c|l|l|}
\hline $\begin{array}{c}\text { Peak } \\
\#\end{array}$ & $\begin{array}{c}\text { RetTime } \\
(\mathrm{min})\end{array}$ & $\begin{array}{c}\text { Area } \\
(\mathrm{mV} * \mathrm{~S})\end{array}$ & $\begin{array}{c}\text { Height } \\
(\mathrm{mV})\end{array}$ & $\begin{array}{c}\text { Area } \\
(\%)\end{array}$ \\
\hline 1 & 17.088 & 16560067 & 396726 & 91.194 \\
\hline 2 & 36.421 & 1599167 & 18380 & 8.806 \\
\hline
\end{tabular}


3ah

$\mathrm{mV}$

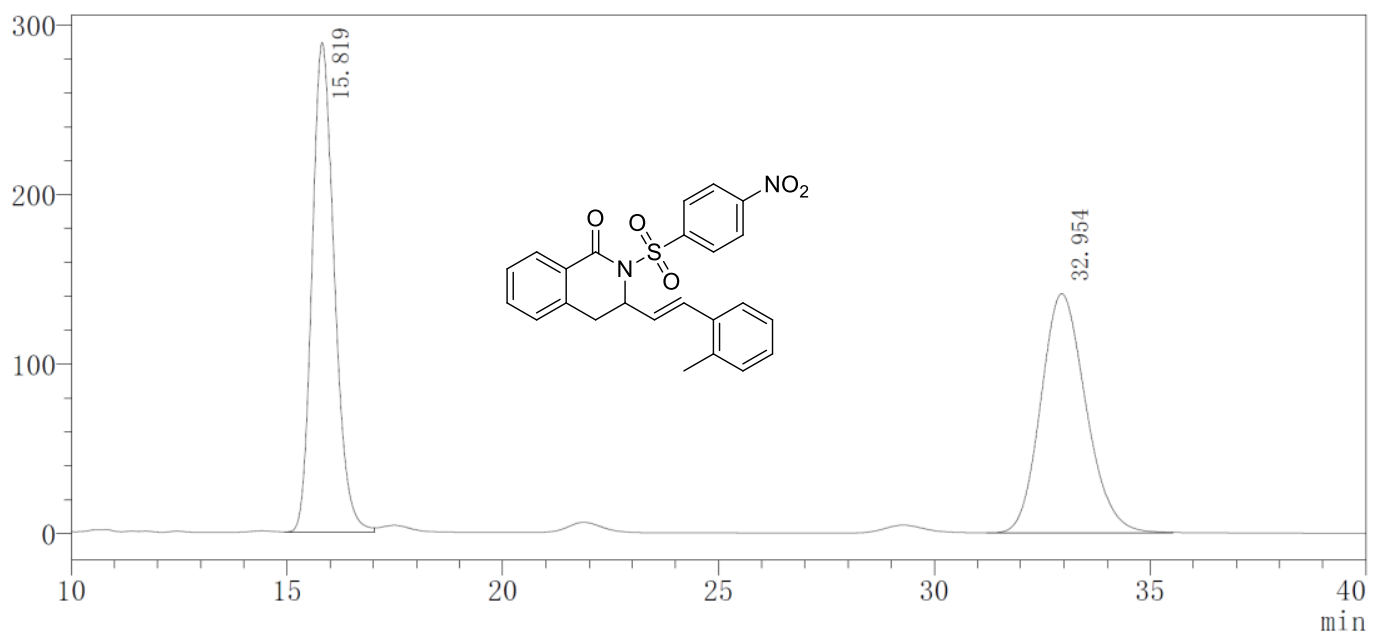

\begin{tabular}{|c|l|c|l|c|}
\hline $\begin{array}{c}\text { Peak } \\
\#\end{array}$ & $\begin{array}{c}\text { RetTime } \\
(\mathrm{min})\end{array}$ & $\begin{array}{c}\text { Area } \\
(\mathrm{mV} * \mathrm{~S})\end{array}$ & $\begin{array}{c}\text { Height } \\
(\mathrm{mV})\end{array}$ & $\begin{array}{c}\text { Area } \\
(\%)\end{array}$ \\
\hline 1 & 15.819 & 10291572 & 288899 & 50.058 \\
\hline 2 & 32.954 & 10267830 & 141133 & 49.942 \\
\hline $\mathrm{mV}$
\end{tabular}

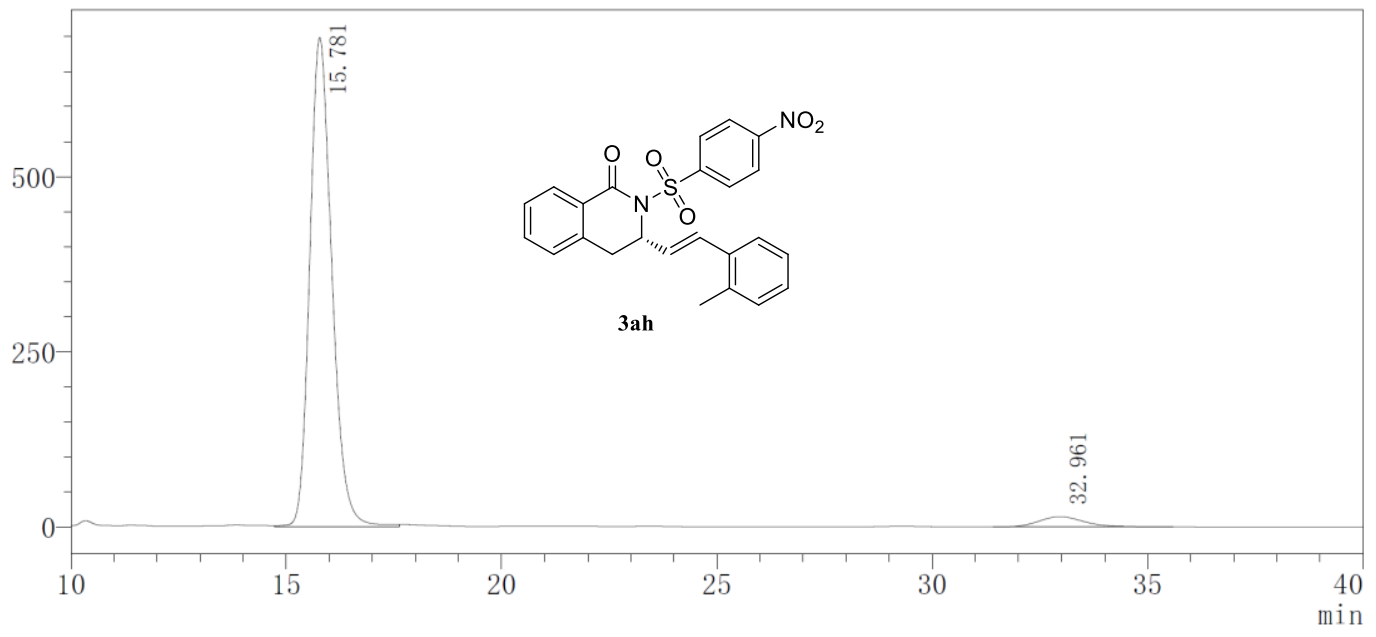

\begin{tabular}{|c|l|l|l|l|}
\hline $\begin{array}{c}\text { Peak } \\
\#\end{array}$ & $\begin{array}{c}\text { RetTime } \\
(\mathrm{min})\end{array}$ & $\begin{array}{c}\text { Area } \\
(\mathrm{mV} * \mathrm{~S})\end{array}$ & $\begin{array}{c}\text { Height } \\
(\mathrm{mV})\end{array}$ & $\begin{array}{c}\text { Area } \\
(\%)\end{array}$ \\
\hline 1 & 15.781 & 24804965 & 698805 & 95.747 \\
\hline 2 & 32.961 & 1101744 & 14797 & 4.253 \\
\hline
\end{tabular}


$3 a i$

mV

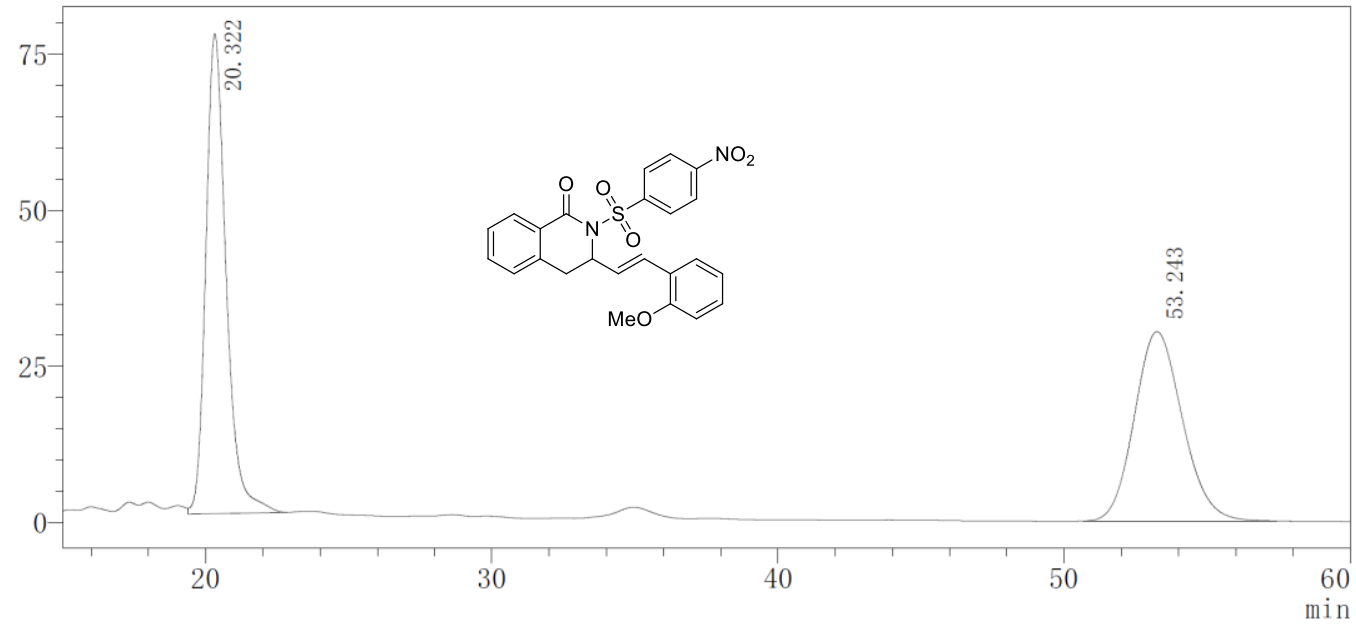

\begin{tabular}{|c|c|c|c|c|}
\hline $\begin{array}{c}\text { Peak } \\
\#\end{array}$ & $\begin{array}{c}\text { RetTime } \\
(\mathrm{min})\end{array}$ & $\begin{array}{c}\text { Area } \\
(\mathrm{mV} * \mathrm{~S})\end{array}$ & $\begin{array}{c}\text { Height } \\
(\mathrm{mV})\end{array}$ & $\begin{array}{c}\text { Area } \\
(\%)\end{array}$ \\
\hline 1 & 20.322 & 3802690 & 76920 & 51.793 \\
\hline 2 & 53.243 & 3539371 & 30348 & 48.207 \\
\hline
\end{tabular}

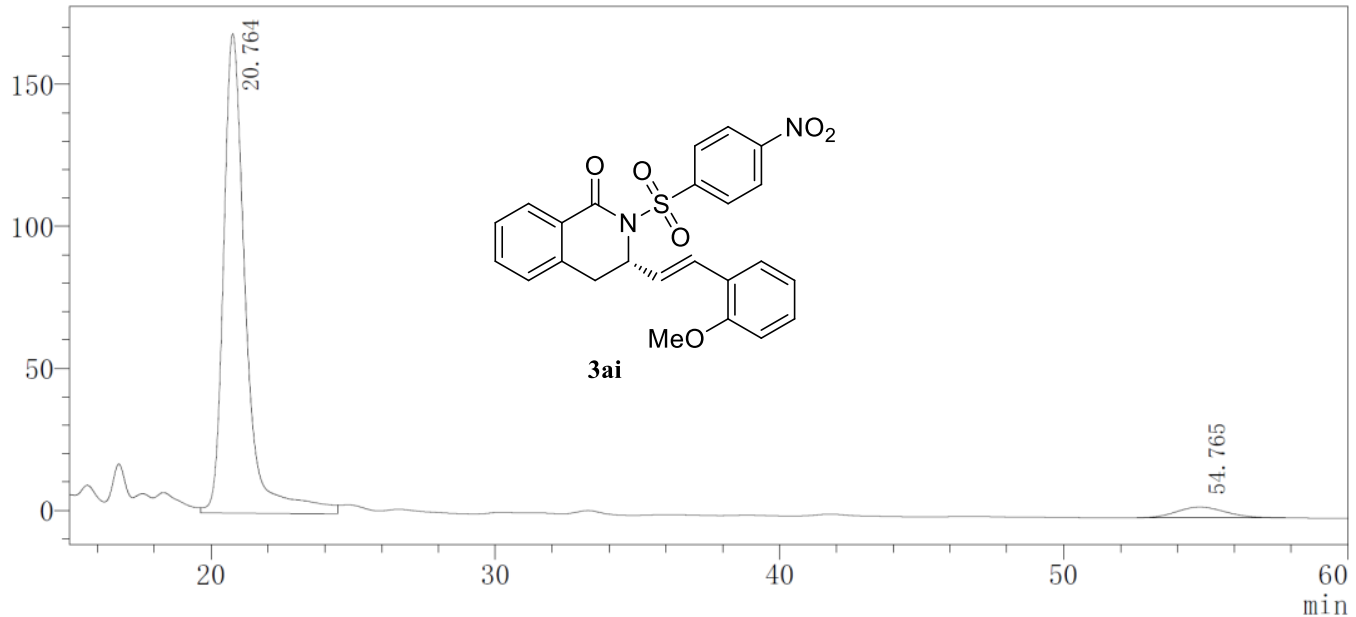

\begin{tabular}{|c|l|l|l|l|}
\hline $\begin{array}{c}\text { Peak } \\
\#\end{array}$ & $\begin{array}{c}\text { RetTime } \\
(\mathrm{min})\end{array}$ & $\begin{array}{c}\text { Area } \\
(\mathrm{mV} * \mathrm{~S})\end{array}$ & $\begin{array}{c}\text { Height } \\
(\mathrm{mV})\end{array}$ & $\begin{array}{c}\text { Area } \\
(\%)\end{array}$ \\
\hline 1 & 20.764 & 9105599 & 168847 & 95.139 \\
\hline 2 & 54.765 & 465231 & 3861 & 4.861 \\
\hline
\end{tabular}


3aj

mV

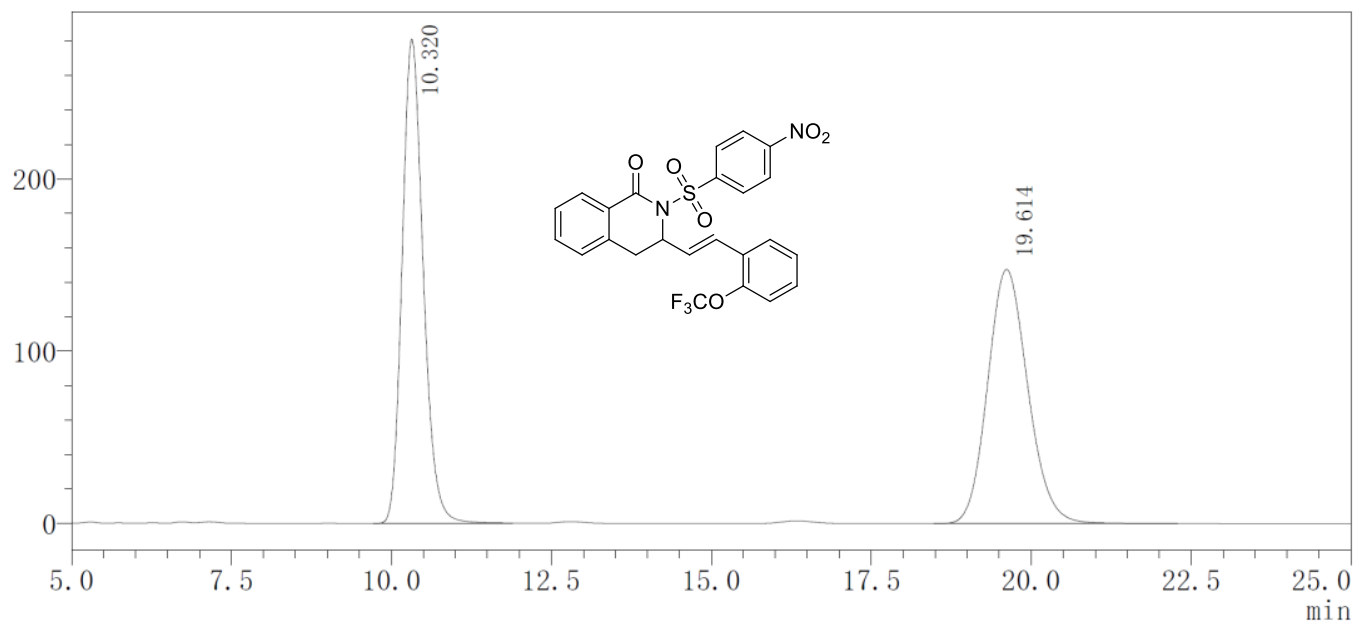

\begin{tabular}{|c|c|c|c|c|}
\hline $\begin{array}{c}\text { Peak } \\
\#\end{array}$ & $\begin{array}{c}\text { RetTime } \\
(\mathrm{min})\end{array}$ & $\begin{array}{c}\text { Area } \\
(\mathrm{mV} * \mathrm{~S})\end{array}$ & $\begin{array}{c}\text { Height } \\
(\mathrm{mV})\end{array}$ & $\begin{array}{c}\text { Area } \\
(\%)\end{array}$ \\
\hline 1 & 10.320 & 6452387 & 280917 & 49.983 \\
\hline 2 & 19.614 & 6456716 & 147403 & 50.017 \\
\hline
\end{tabular}

$\mathrm{mV}$

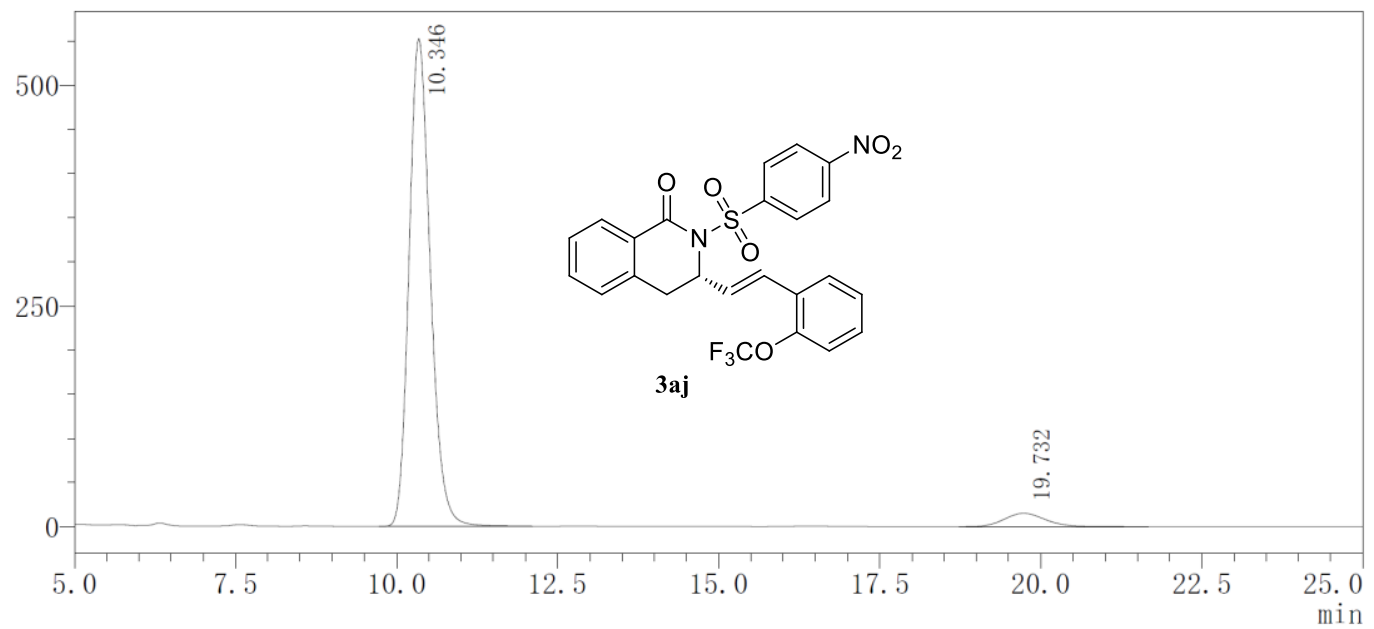

\begin{tabular}{|c|c|c|c|c|}
\hline $\begin{array}{c}\text { Peak } \\
\#\end{array}$ & $\begin{array}{c}\text { RetTime } \\
(\mathrm{min})\end{array}$ & $\begin{array}{c}\text { Area } \\
(\mathrm{mV} * \mathrm{~S})\end{array}$ & $\begin{array}{c}\text { Height } \\
(\mathrm{mV})\end{array}$ & $\begin{array}{c}\text { Area } \\
(\%)\end{array}$ \\
\hline 1 & 10.346 & 12737010 & 552316 & 94.963 \\
\hline 2 & 19.732 & 675633 & 15158 & 5.037 \\
\hline
\end{tabular}


3ak

$\mathrm{mV}$

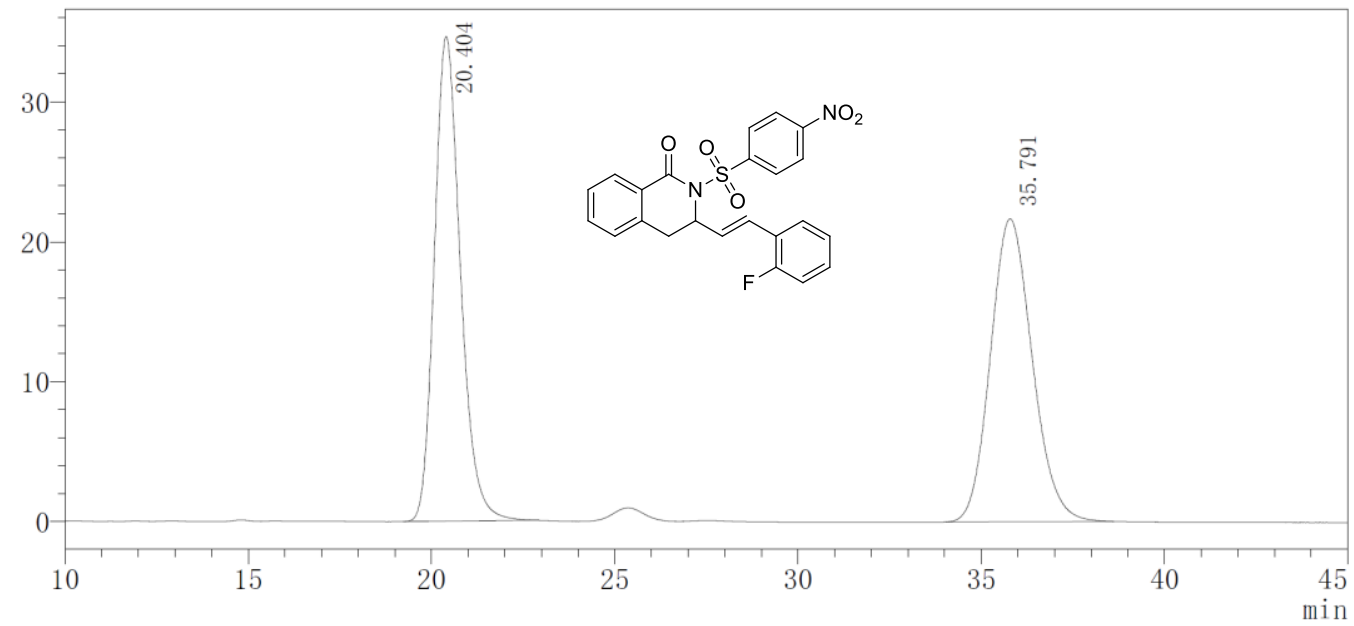

\begin{tabular}{|c|c|c|c|c|}
\hline $\begin{array}{c}\text { Peak } \\
\#\end{array}$ & $\begin{array}{c}\text { RetTime } \\
(\mathrm{min})\end{array}$ & $\begin{array}{c}\text { Area } \\
(\mathrm{mV} * \mathrm{~S})\end{array}$ & $\begin{array}{c}\text { Height } \\
(\mathrm{mV})\end{array}$ & $\begin{array}{c}\text { Area } \\
(\%)\end{array}$ \\
\hline 1 & 20.404 & 1715198 & 34627 & 49.963 \\
\hline 2 & 35.791 & 1717736 & 21644 & 50.037 \\
\hline
\end{tabular}

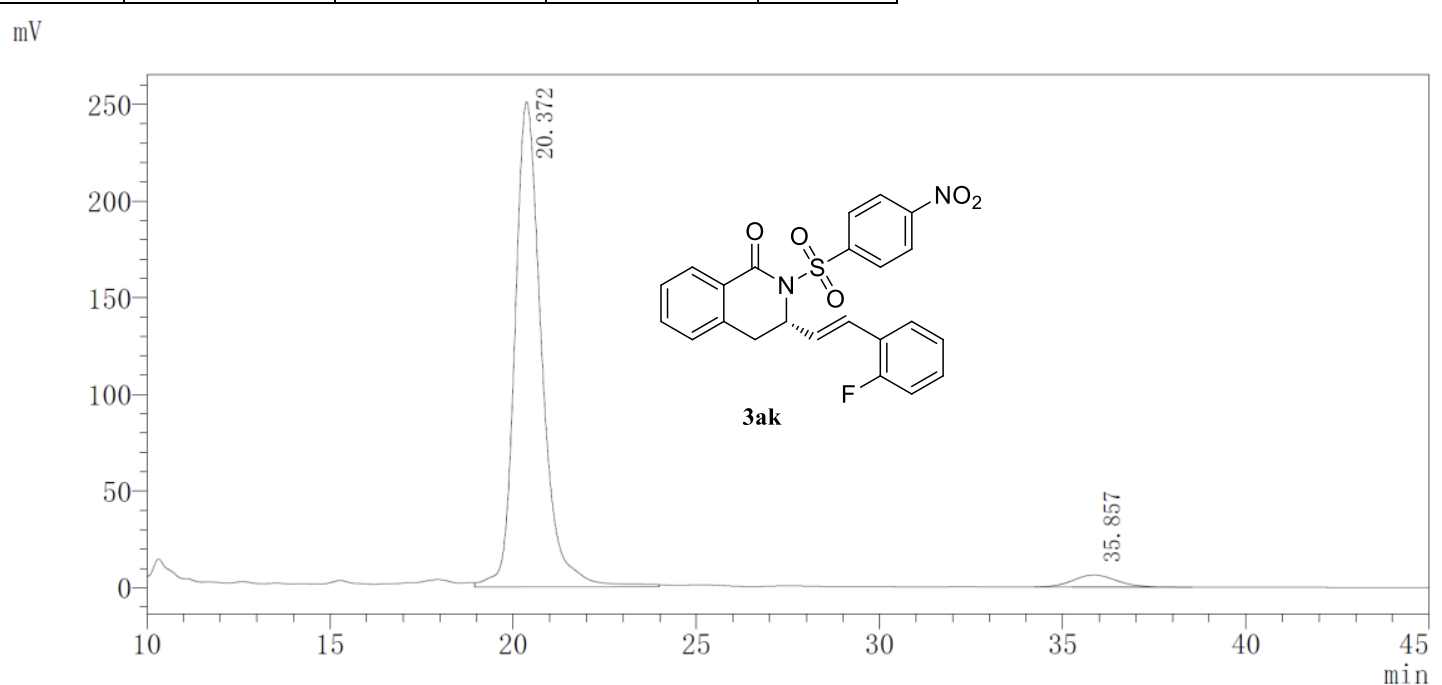

\begin{tabular}{|c|l|l|l|l|}
\hline $\begin{array}{c}\text { Peak } \\
\#\end{array}$ & $\begin{array}{c}\text { RetTime } \\
(\mathrm{min})\end{array}$ & $\begin{array}{c}\text { Area } \\
(\mathrm{mV} * \mathrm{~S})\end{array}$ & $\begin{array}{c}\text { Height } \\
(\mathrm{mV})\end{array}$ & $\begin{array}{c}\text { Area } \\
(\%)\end{array}$ \\
\hline 1 & 20.372 & 12729185 & 250824 & 96.123 \\
\hline 2 & 35.857 & 513473 & 6260 & 3.877 \\
\hline
\end{tabular}




\section{3al}

$\mathrm{mV}$

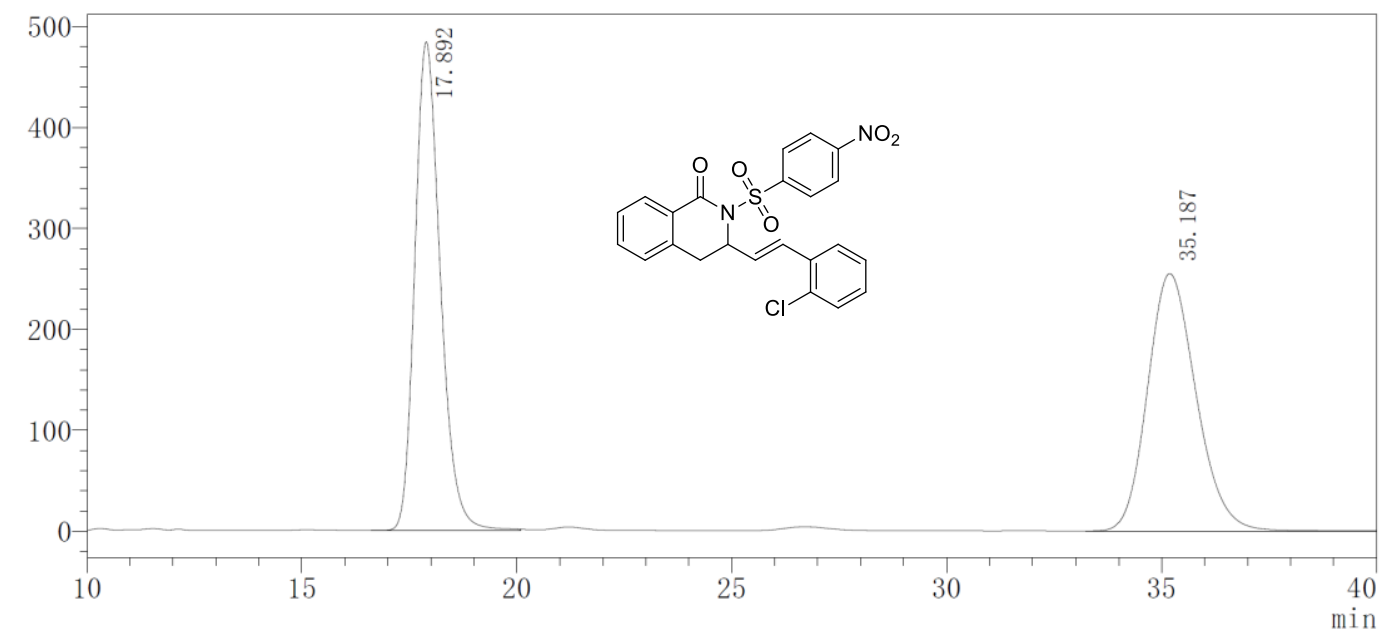

\begin{tabular}{|c|c|c|c|c|}
\hline $\begin{array}{c}\text { Peak } \\
\#\end{array}$ & $\begin{array}{c}\text { RetTime } \\
(\mathrm{min})\end{array}$ & $\begin{array}{c}\text { Area } \\
(\mathrm{mV} * \mathrm{~S})\end{array}$ & $\begin{array}{c}\text { Height } \\
(\mathrm{mV})\end{array}$ & $\begin{array}{c}\text { Area } \\
(\%)\end{array}$ \\
\hline 1 & 17.892 & 20045265 & 484124 & 50.022 \\
\hline 2 & 35.187 & 20027865 & 255060 & 49.978 \\
\hline
\end{tabular}

$\mathrm{mV}$

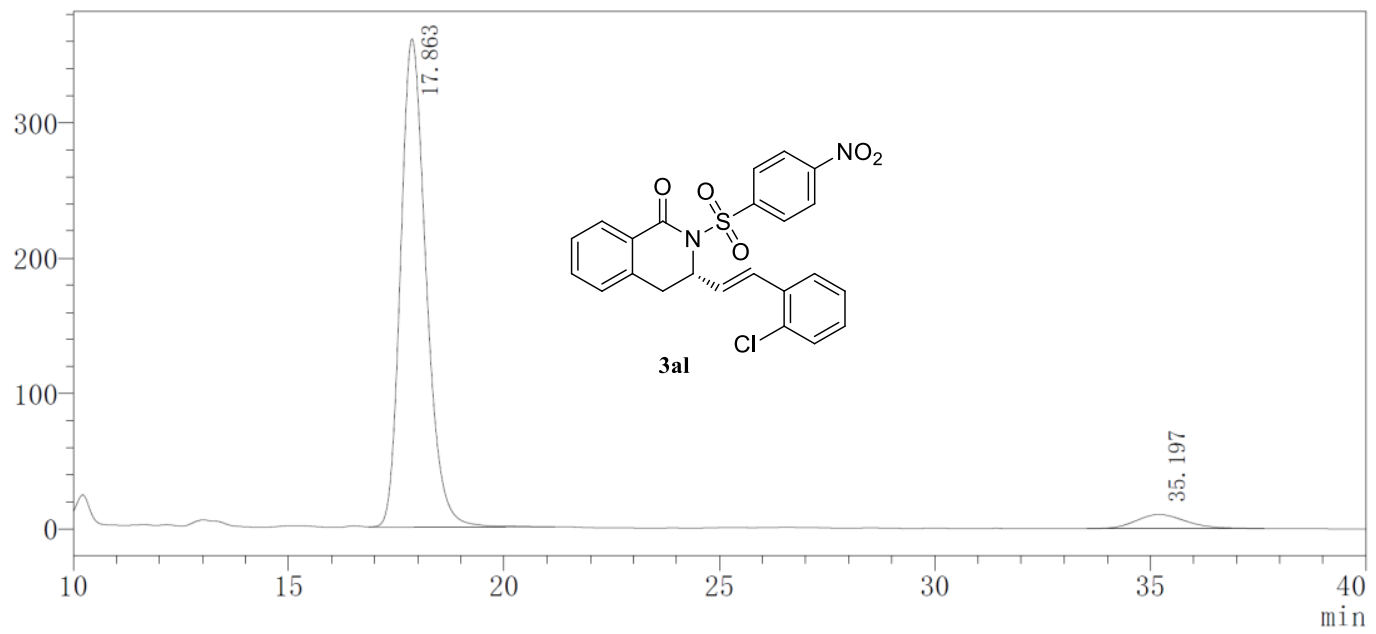

\begin{tabular}{|c|l|l|l|l|}
\hline $\begin{array}{c}\text { Peak } \\
\#\end{array}$ & $\begin{array}{c}\text { RetTime } \\
(\mathrm{min})\end{array}$ & $\begin{array}{c}\text { Area } \\
(\mathrm{mV} * \mathrm{~S})\end{array}$ & $\begin{array}{c}\text { Height } \\
(\mathrm{mV})\end{array}$ & $\begin{array}{c}\text { Area } \\
(\%)\end{array}$ \\
\hline 1 & 17.863 & 14849309 & 360570 & 94.628 \\
\hline 2 & 35.197 & 842915 & 10463 & 5.372 \\
\hline
\end{tabular}


3 am

$\mathrm{mV}$

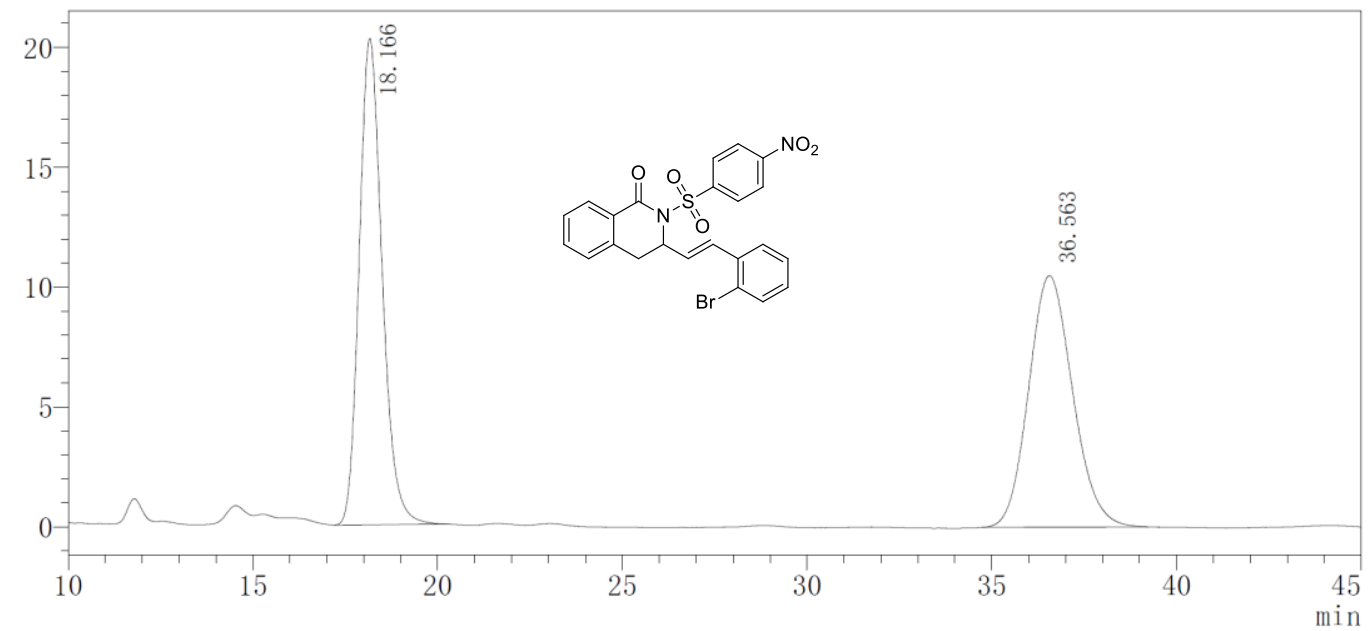

\begin{tabular}{|c|c|c|l|c|}
\hline $\begin{array}{c}\text { Peak } \\
\#\end{array}$ & $\begin{array}{c}\text { RetTime } \\
(\mathrm{min})\end{array}$ & $\begin{array}{c}\text { Area } \\
(\mathrm{mV} * \mathrm{~S})\end{array}$ & $\begin{array}{c}\text { Height } \\
(\mathrm{mV})\end{array}$ & $\begin{array}{c}\text { Area } \\
(\%)\end{array}$ \\
\hline 1 & 18.166 & 877031 & 20278 & 50.260 \\
\hline 2 & 36.563 & 867969 & 10492 & 49.740 \\
\hline
\end{tabular}

$\mathrm{mV}$

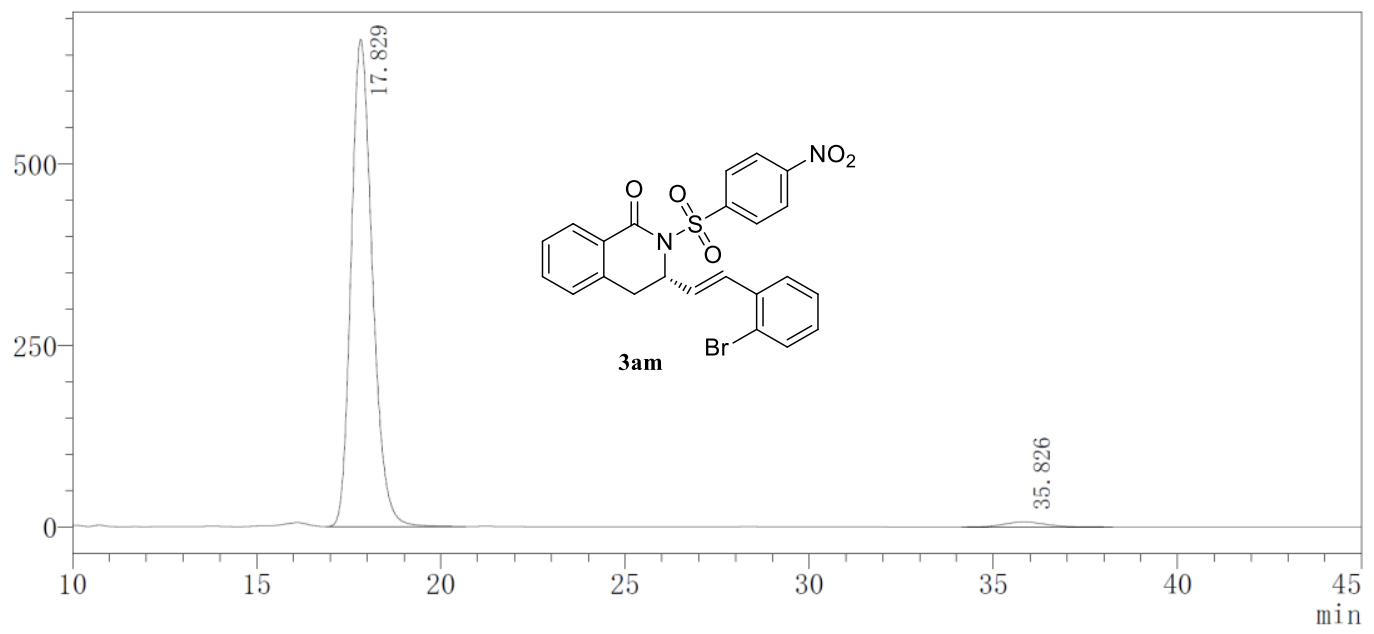

\begin{tabular}{|c|l|l|l|l|}
\hline $\begin{array}{c}\text { Peak } \\
\#\end{array}$ & $\begin{array}{c}\text { RetTime } \\
(\mathrm{min})\end{array}$ & $\begin{array}{c}\text { Area } \\
(\mathrm{mV} * \mathrm{~S})\end{array}$ & $\begin{array}{c}\text { Height } \\
(\mathrm{mV})\end{array}$ & $\begin{array}{c}\text { Area } \\
(\%)\end{array}$ \\
\hline 1 & 17.829 & 27017090 & 671116 & 97.966 \\
\hline 2 & 35.826 & 560898 & 6992 & 2.034 \\
\hline
\end{tabular}


3 an

$\mathrm{mV}$

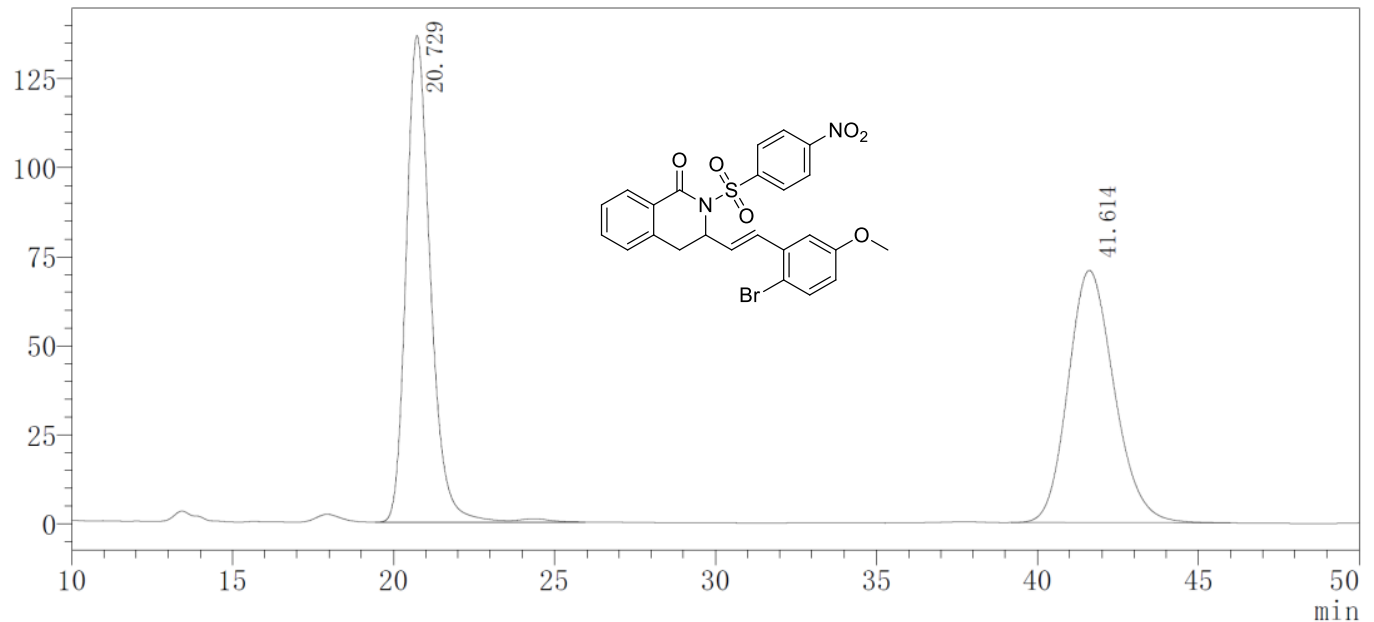

\begin{tabular}{|c|c|c|c|c|}
\hline $\begin{array}{c}\text { Peak } \\
\#\end{array}$ & $\begin{array}{c}\text { RetTime } \\
(\mathrm{min})\end{array}$ & $\begin{array}{c}\text { Area } \\
(\mathrm{mV} * \mathrm{~S})\end{array}$ & $\begin{array}{c}\text { Height } \\
(\mathrm{mV})\end{array}$ & $\begin{array}{c}\text { Area } \\
(\%)\end{array}$ \\
\hline 1 & 20.729 & 7213853 & 136612 & 50.510 \\
\hline 2 & 41.614 & 7068072 & 70876 & 49.490 \\
\hline
\end{tabular}

$\mathrm{mV}$

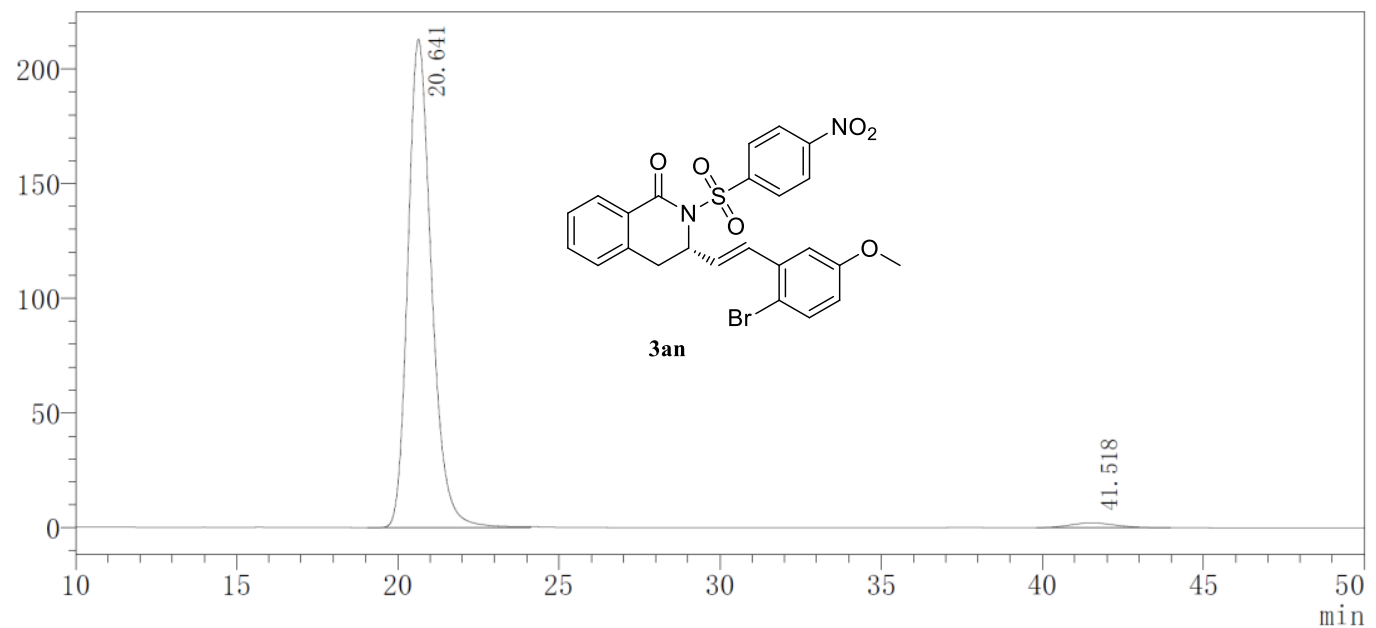

\begin{tabular}{|c|l|l|l|l|}
\hline $\begin{array}{c}\text { Peak } \\
\#\end{array}$ & $\begin{array}{c}\text { RetTime } \\
(\mathrm{min})\end{array}$ & $\begin{array}{c}\text { Area } \\
(\mathrm{mV} * \mathrm{~S})\end{array}$ & $\begin{array}{c}\text { Height } \\
(\mathrm{mV})\end{array}$ & $\begin{array}{c}\text { Area } \\
(\%)\end{array}$ \\
\hline 1 & 20.641 & 10931544 & 212919 & 98.142 \\
\hline 2 & 41.518 & 206945 & 2119 & 1.858 \\
\hline
\end{tabular}


3 ao

$\mathrm{mV}$

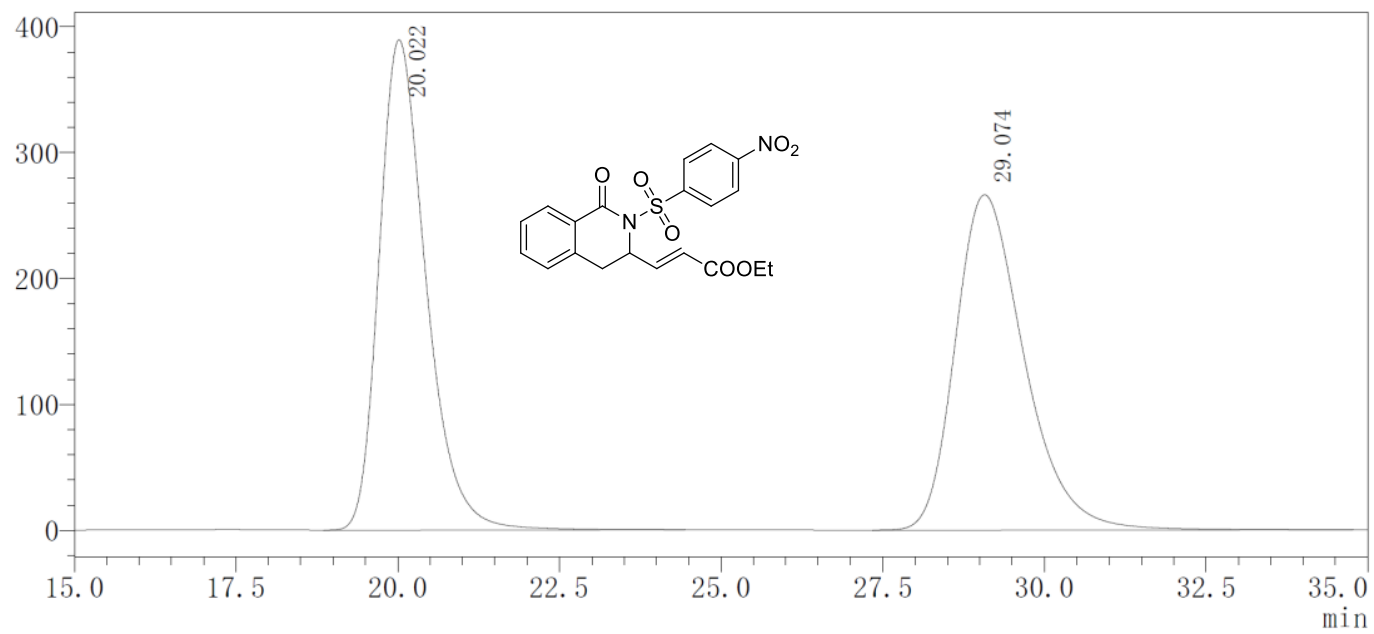

\begin{tabular}{|c|c|c|c|c|}
\hline $\begin{array}{c}\text { Peak } \\
\#\end{array}$ & $\begin{array}{c}\text { RetTime } \\
(\mathrm{min})\end{array}$ & $\begin{array}{c}\text { Area } \\
(\mathrm{mV} * \mathrm{~S})\end{array}$ & $\begin{array}{c}\text { Height } \\
(\mathrm{mV})\end{array}$ & $\begin{array}{c}\text { Area } \\
(\%)\end{array}$ \\
\hline 1 & 20.022 & 19659691 & 389297 & 49.852 \\
\hline 2 & 29.074 & 19776116 & 266318 & 50.148 \\
\hline
\end{tabular}

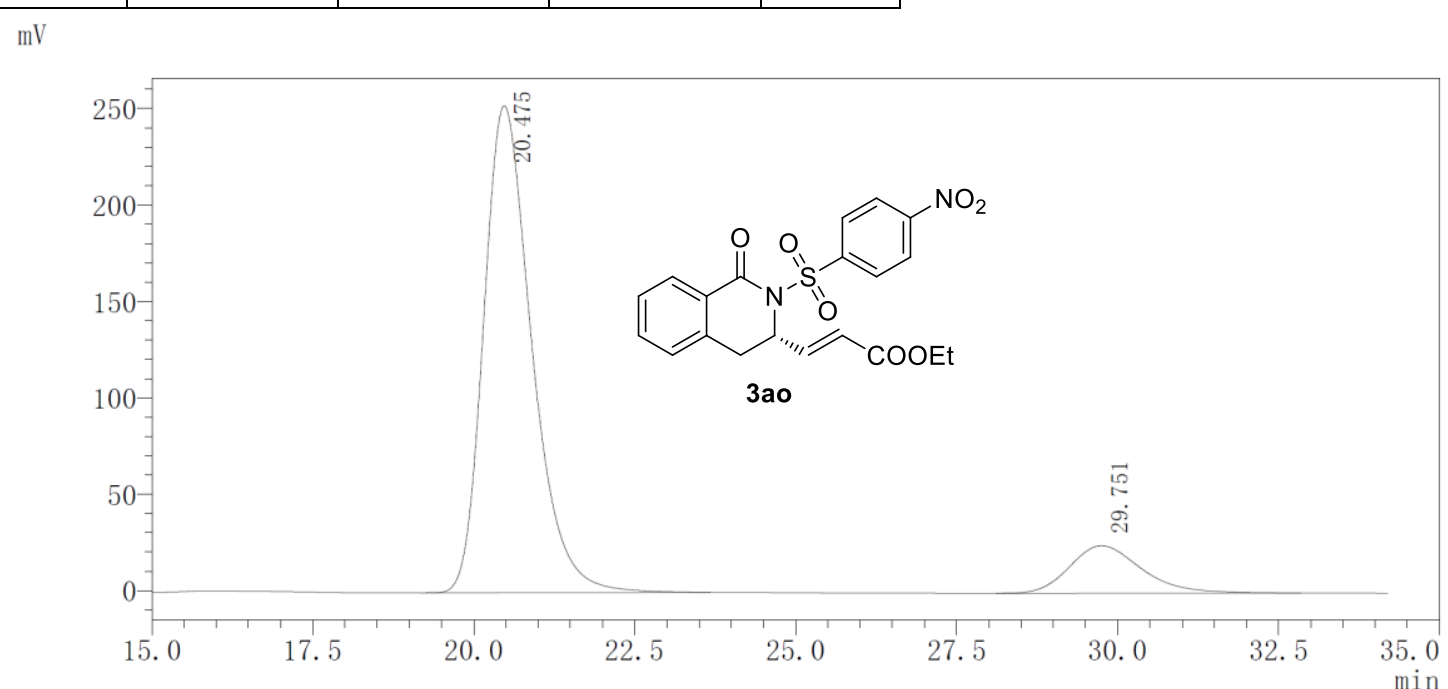

\begin{tabular}{|c|l|l|l|l|}
\hline $\begin{array}{c}\text { Peak } \\
\#\end{array}$ & $\begin{array}{c}\text { RetTime } \\
(\mathrm{min})\end{array}$ & $\begin{array}{c}\text { Area } \\
(\mathrm{mV} * \mathrm{~S})\end{array}$ & $\begin{array}{c}\text { Height } \\
(\mathrm{mV})\end{array}$ & $\begin{array}{c}\text { Area } \\
(\%)\end{array}$ \\
\hline 1 & 20.475 & 13124463 & 252401 & 87.247 \\
\hline 2 & 29.751 & 1918389 & 24611 & 12.753 \\
\hline
\end{tabular}


3 ap

$\mathrm{mV}$

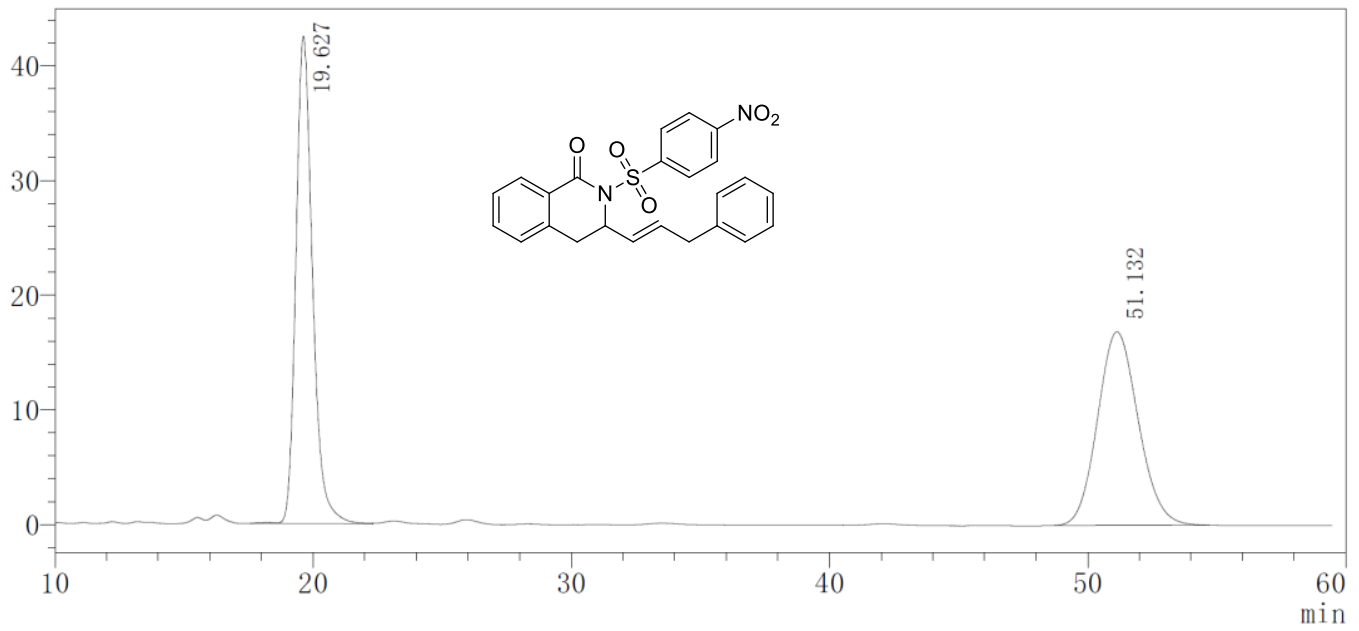

\begin{tabular}{|c|l|l|l|l|}
\hline $\begin{array}{c}\text { Peak } \\
\#\end{array}$ & $\begin{array}{c}\text { RetTime } \\
(\mathrm{min})\end{array}$ & $\begin{array}{c}\text { Area } \\
(\mathrm{mV} * \mathrm{~S})\end{array}$ & $\begin{array}{c}\text { Height } \\
(\mathrm{mV})\end{array}$ & $\begin{array}{c}\text { Area } \\
(\%)\end{array}$ \\
\hline 1 & 19.627 & 1905823 & 42485 & 50.707 \\
\hline 2 & 51.132 & 1852702 & 16877 & 49.293 \\
\hline
\end{tabular}

$\mathrm{mV}$

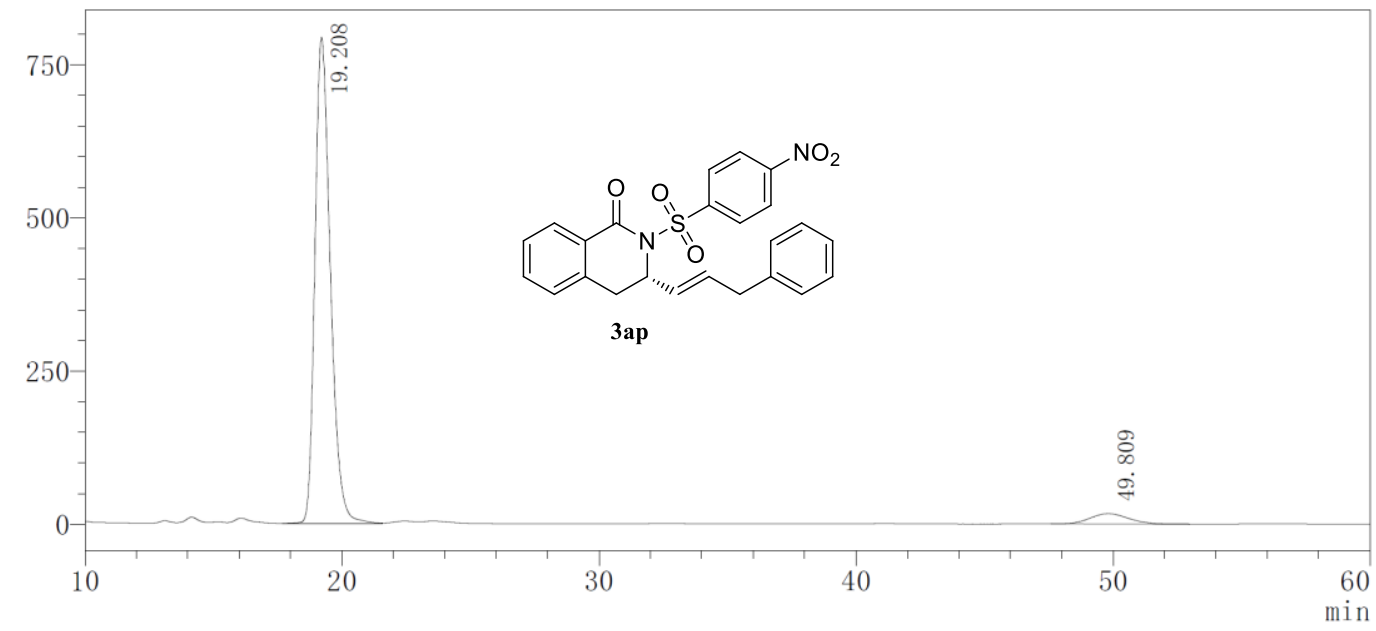

\begin{tabular}{|c|l|l|l|l|}
\hline $\begin{array}{c}\text { Peak } \\
\#\end{array}$ & $\begin{array}{c}\text { RetTime } \\
(\mathrm{min})\end{array}$ & $\begin{array}{c}\text { Area } \\
(\mathrm{mV} * \mathrm{~S})\end{array}$ & $\begin{array}{c}\text { Height } \\
(\mathrm{mV})\end{array}$ & $\begin{array}{c}\text { Area } \\
(\%)\end{array}$ \\
\hline 1 & 19.208 & 33352846 & 794275 & 94.918 \\
\hline 2 & 49.809 & 1785625 & 16972 & 5.082 \\
\hline
\end{tabular}


$3 a q$

$\mathrm{mV}$

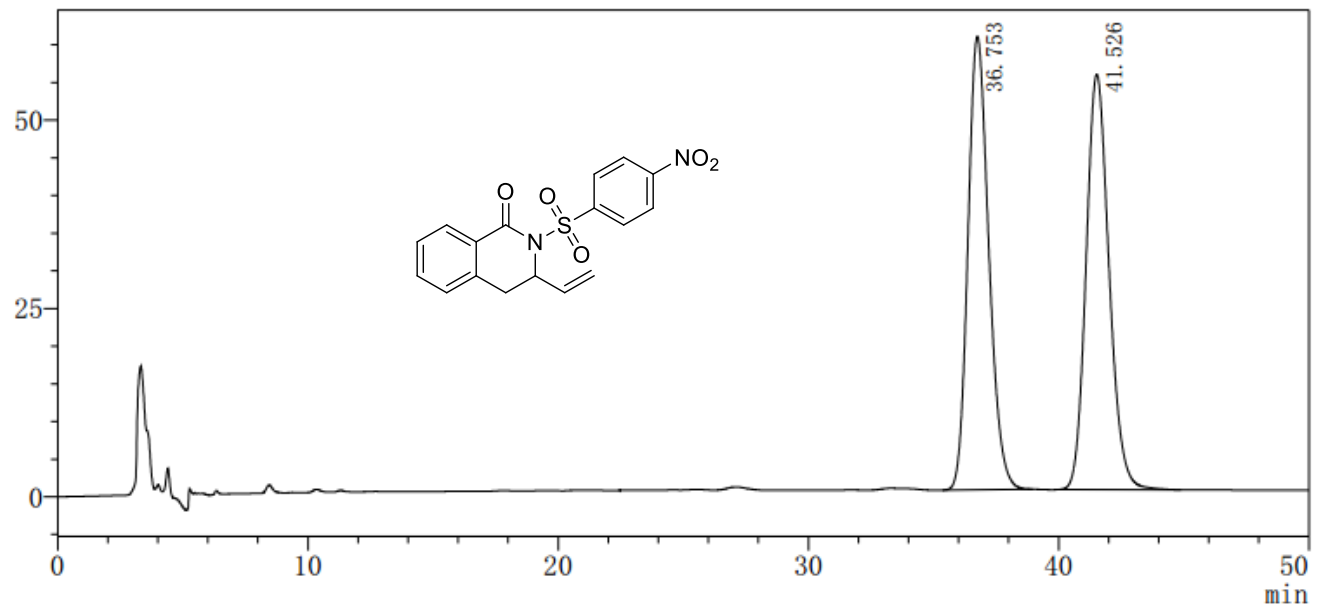

\begin{tabular}{|c|c|c|c|c|}
\hline $\begin{array}{c}\text { Peak } \\
\#\end{array}$ & $\begin{array}{c}\text { RetTime } \\
(\mathrm{min})\end{array}$ & $\begin{array}{c}\text { Area } \\
(\mathrm{mV} * \mathrm{~S})\end{array}$ & $\begin{array}{c}\text { Height } \\
(\mathrm{mV})\end{array}$ & $\begin{array}{c}\text { Area } \\
(\%)\end{array}$ \\
\hline 1 & 36.753 & 3484446 & 60167 & 49.741 \\
\hline 2 & 41.526 & 3520706 & 55169 & 50.259 \\
\hline
\end{tabular}

$\mathrm{mV}$

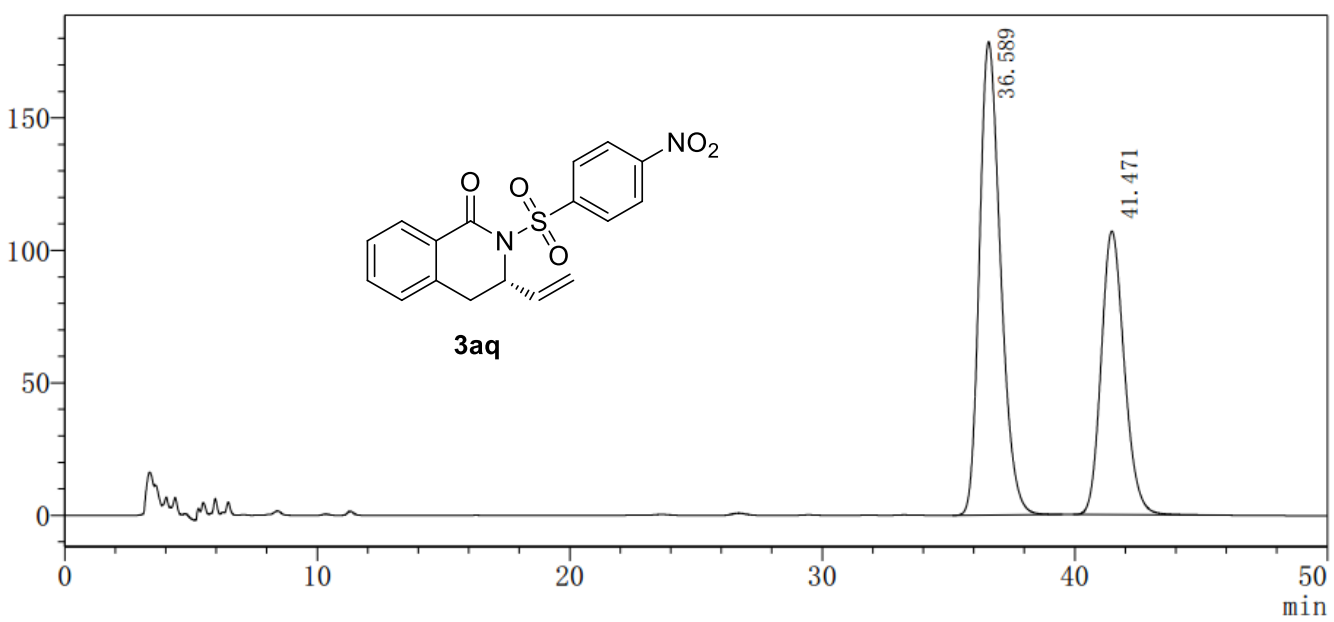

\begin{tabular}{|c|c|c|c|c|}
\hline $\begin{array}{c}\text { Peak } \\
\#\end{array}$ & $\begin{array}{c}\text { RetTime } \\
(\mathrm{min})\end{array}$ & $\begin{array}{c}\text { Area } \\
(\mathrm{mV} * \mathrm{~S})\end{array}$ & $\begin{array}{c}\text { Height } \\
(\mathrm{mV})\end{array}$ & $\begin{array}{c}\text { Area } \\
(\%)\end{array}$ \\
\hline 1 & 36.589 & 10401691 & 178616 & 60.444 \\
\hline 2 & 41.471 & 6807087 & 107071 & 39.556 \\
\hline
\end{tabular}




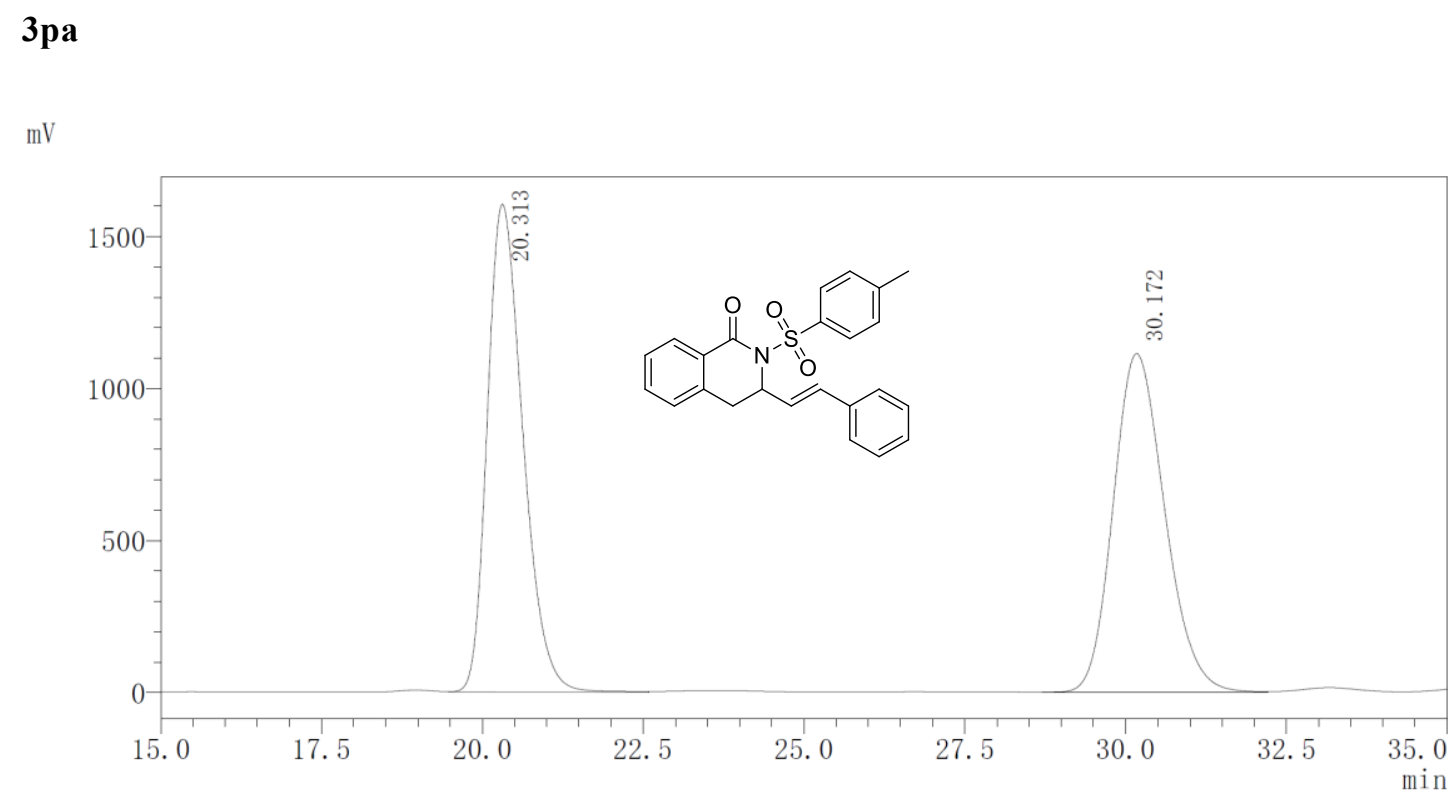

\begin{tabular}{|c|l|c|c|c|}
\hline $\begin{array}{c}\text { Peak } \\
\#\end{array}$ & $\begin{array}{c}\text { RetTime } \\
(\mathrm{min})\end{array}$ & $\begin{array}{c}\text { Area } \\
(\mathrm{mV} * \mathrm{~S})\end{array}$ & $\begin{array}{c}\text { Height } \\
(\mathrm{mV})\end{array}$ & $\begin{array}{c}\text { Area } \\
(\%)\end{array}$ \\
\hline 1 & 20.313 & 61825915 & 1605127 & 49.896 \\
\hline 2 & 30.172 & 62084236 & 1113900 & 50.104 \\
\hline $\mathrm{mV}$
\end{tabular}

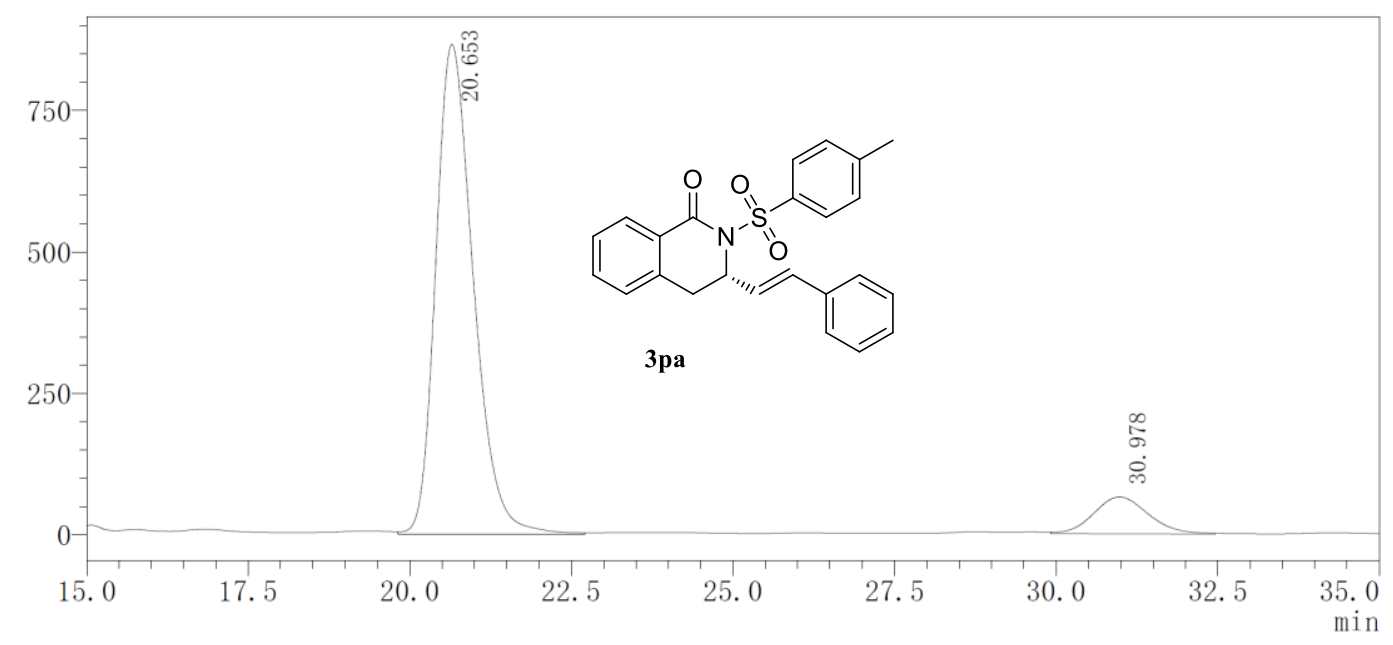

\begin{tabular}{|c|l|l|l|l|}
\hline $\begin{array}{c}\text { Peak } \\
\#\end{array}$ & $\begin{array}{c}\text { RetTime } \\
(\mathrm{min})\end{array}$ & $\begin{array}{c}\text { Area } \\
(\mathrm{mV} * \mathrm{~S})\end{array}$ & $\begin{array}{c}\text { Height } \\
(\mathrm{mV})\end{array}$ & $\begin{array}{c}\text { Area } \\
(\%)\end{array}$ \\
\hline 1 & 20.653 & 35103875 & 866416 & 90.188 \\
\hline 2 & 30.978 & 3819051 & 64951 & 9.812 \\
\hline
\end{tabular}




\section{$3 q a$}

$\mathrm{mV}$

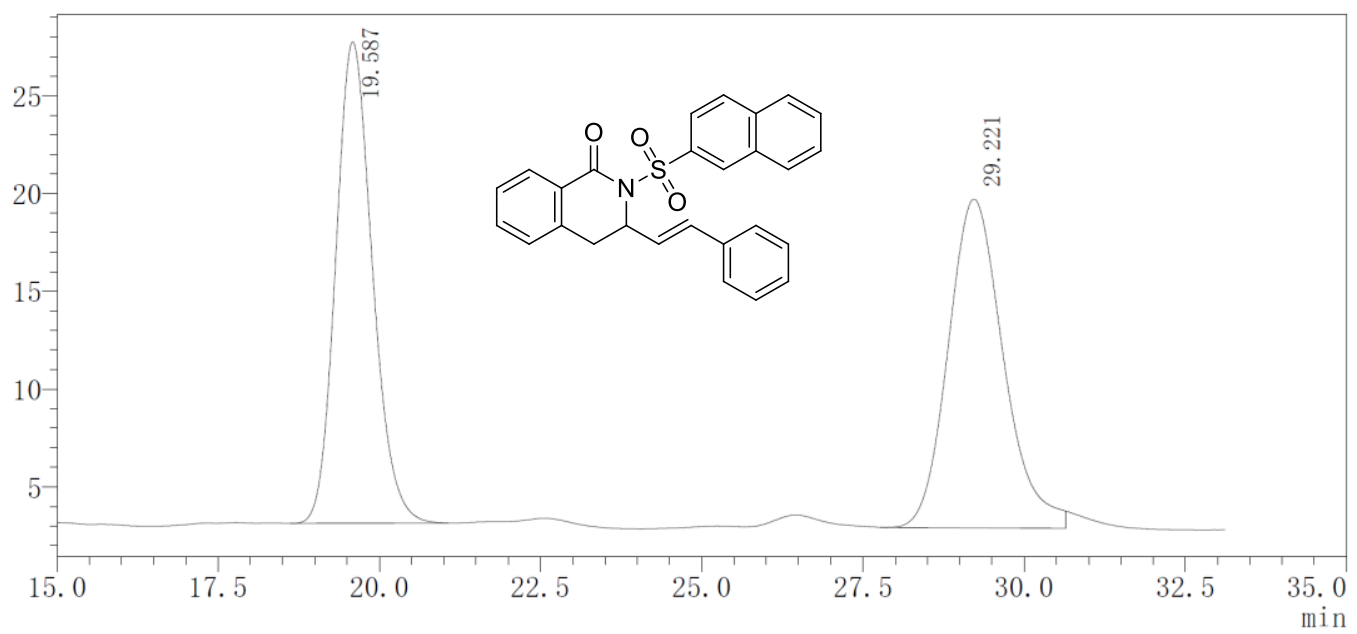

\begin{tabular}{|c|c|c|c|c|}
\hline $\begin{array}{c}\text { Peak } \\
\#\end{array}$ & $\begin{array}{c}\text { RetTime } \\
(\mathrm{min})\end{array}$ & $\begin{array}{c}\text { Area } \\
(\mathrm{mV} * \mathrm{~S})\end{array}$ & $\begin{array}{c}\text { Height } \\
(\mathrm{mV})\end{array}$ & $\begin{array}{c}\text { Area } \\
(\%)\end{array}$ \\
\hline 1 & 19.587 & 975331 & 24617 & 49.324 \\
\hline 2 & 29.221 & 1002061 & 16820 & 50.676 \\
\hline $\mathrm{mV}$
\end{tabular}

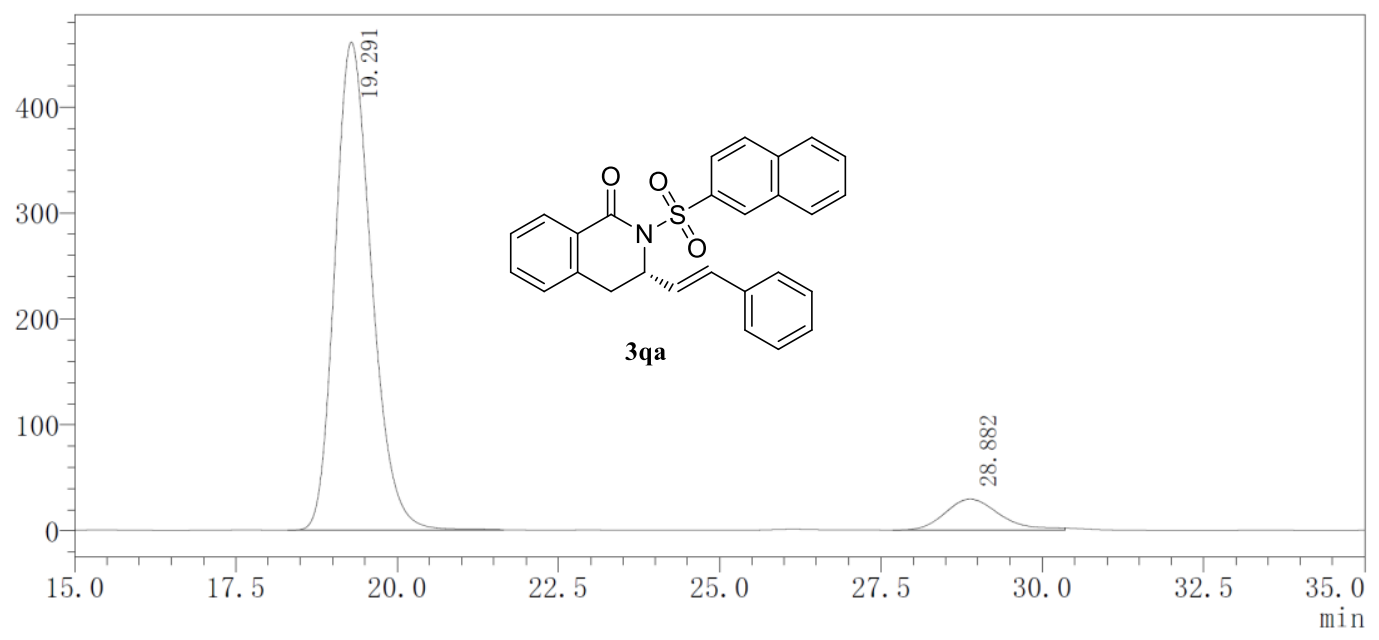

\begin{tabular}{|c|c|c|c|c|}
\hline $\begin{array}{c}\text { Peak } \\
\#\end{array}$ & $\begin{array}{c}\text { RetTime } \\
(\mathrm{min})\end{array}$ & $\begin{array}{c}\text { Area } \\
(\mathrm{mV} * \mathrm{~S})\end{array}$ & $\begin{array}{c}\text { Height } \\
(\mathrm{mV})\end{array}$ & $\begin{array}{c}\text { Area } \\
(\%)\end{array}$ \\
\hline 1 & 19.291 & 18255756 & 460962 & 91.294 \\
\hline 2 & 28.882 & 1740894 & 29271 & 8.706 \\
\hline
\end{tabular}




\section{3ra}

$\mathrm{mV}$

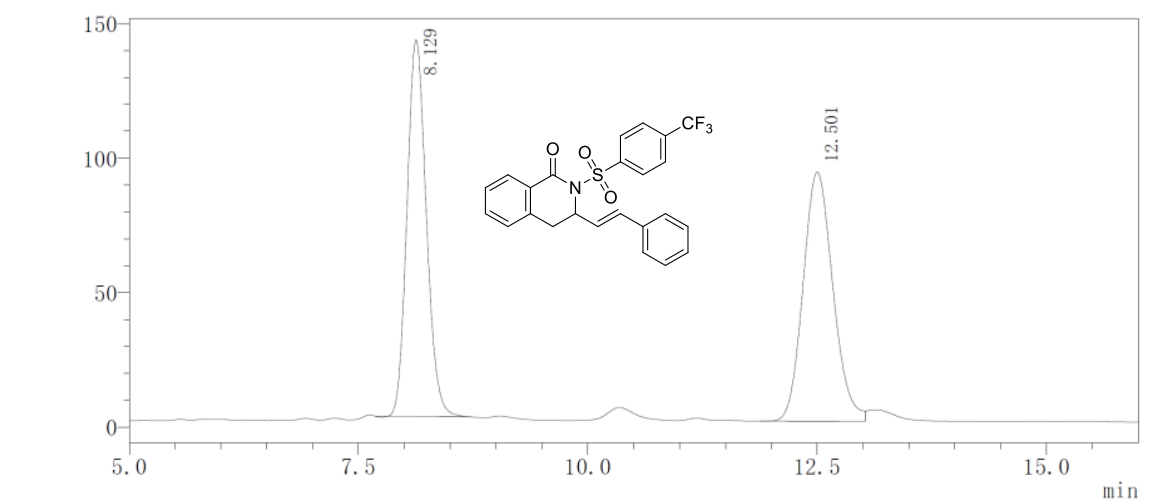

\begin{tabular}{|l|l|l|l|l|}
\hline Peak\# & RetTime $(\min )$ & $\operatorname{Area}(\mathrm{mV} * \mathrm{~S})$ & Height $(\mathrm{mV})$ & $\operatorname{Area}(\%)$ \\
\hline 1 & 8.129 & 2050929 & 140000 & 49.498 \\
\hline 2 & 12.501 & 2092529 & 92591 & 50.502 \\
\hline
\end{tabular}

$\mathrm{mV}$

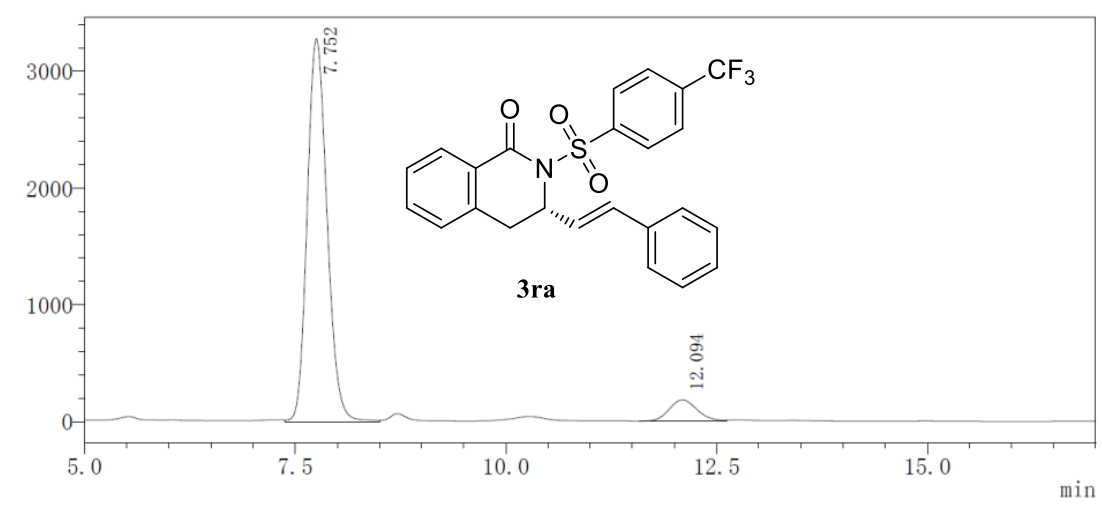

\begin{tabular}{|l|l|l|l|l|}
\hline Peak\# & RetTime $(\mathrm{min})$ & $\operatorname{Area}(\mathrm{mV} * \mathrm{~S})$ & Height $(\mathrm{mV})$ & $\mathrm{Area}(\%)$ \\
\hline 1 & 7.752 & 53755429 & 3278074 & 92.632 \\
\hline 2 & 12.094 & 4275537 & 183351 & 7.368 \\
\hline
\end{tabular}

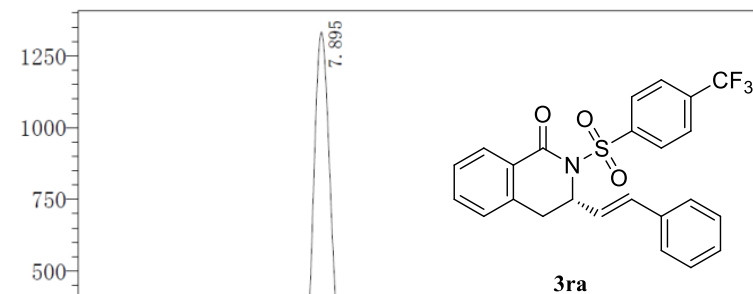

3ra

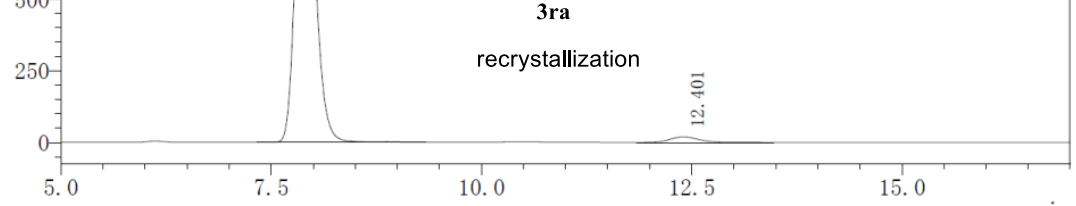

\begin{tabular}{|l|l|l|l|l|}
\hline Peak\# & RetTime $(\mathrm{min})$ & Area $(\mathrm{mV} * \mathrm{~S})$ & Height $(\mathrm{mV})$ & \multicolumn{1}{|c|}{ Area $(\%)$} \\
\hline 1 & 7.895 & 20138080 & 1331900 & 97.772 \\
\hline 2 & 12.401 & 458855 & 19544 & 2.228 \\
\hline
\end{tabular}


3sa

$\mathrm{mV}$

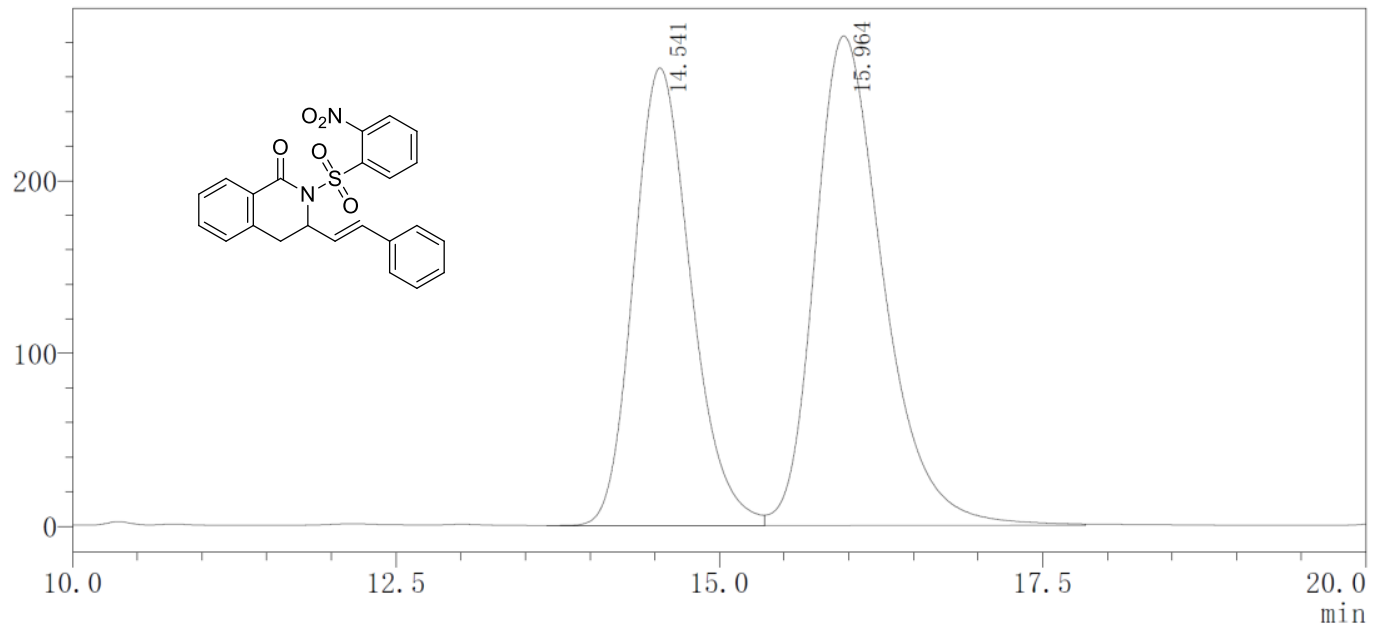

\begin{tabular}{|c|c|c|c|c|}
\hline $\begin{array}{c}\text { Peak } \\
\#\end{array}$ & $\begin{array}{c}\text { RetTime } \\
(\mathrm{min})\end{array}$ & $\begin{array}{c}\text { Area } \\
(\mathrm{mV} * \mathrm{~S})\end{array}$ & $\begin{array}{c}\text { Height } \\
(\mathrm{mV})\end{array}$ & $\begin{array}{c}\text { Area } \\
(\%)\end{array}$ \\
\hline 1 & 14.541 & 8212258 & 265000 & 44.101 \\
\hline 2 & 15.964 & 10409134 & 283441 & 55.899 \\
\hline
\end{tabular}

$\mathrm{mV}$

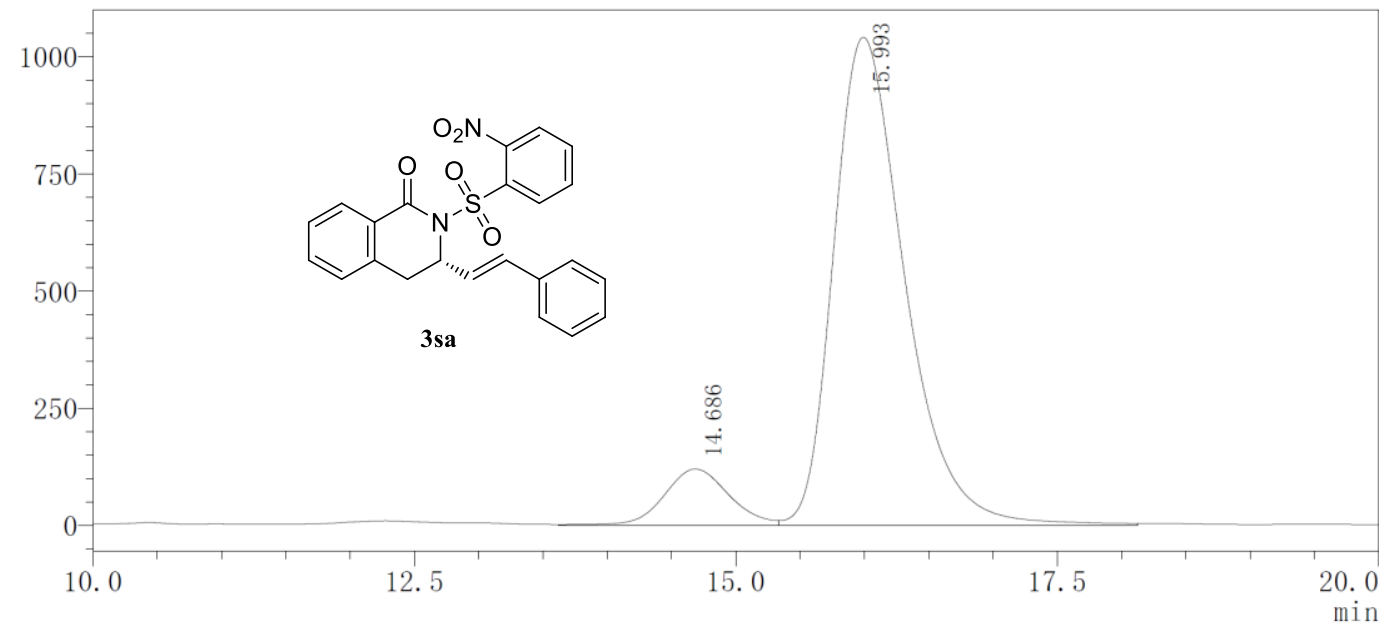

\begin{tabular}{|c|l|l|l|l|}
\hline $\begin{array}{c}\text { Peak } \\
\#\end{array}$ & $\begin{array}{c}\text { RetTime } \\
(\mathrm{min})\end{array}$ & $\begin{array}{c}\text { Area } \\
(\mathrm{mV} * \mathrm{~S})\end{array}$ & $\begin{array}{c}\text { Height } \\
(\mathrm{mV})\end{array}$ & $\begin{array}{c}\text { Area } \\
(\%)\end{array}$ \\
\hline 1 & 14.686 & 4226060 & 120019 & 9.557 \\
\hline 2 & 15.993 & 39995323 & 1040729 & 90.443 \\
\hline
\end{tabular}




\section{3ta}

$\mathrm{mV}$

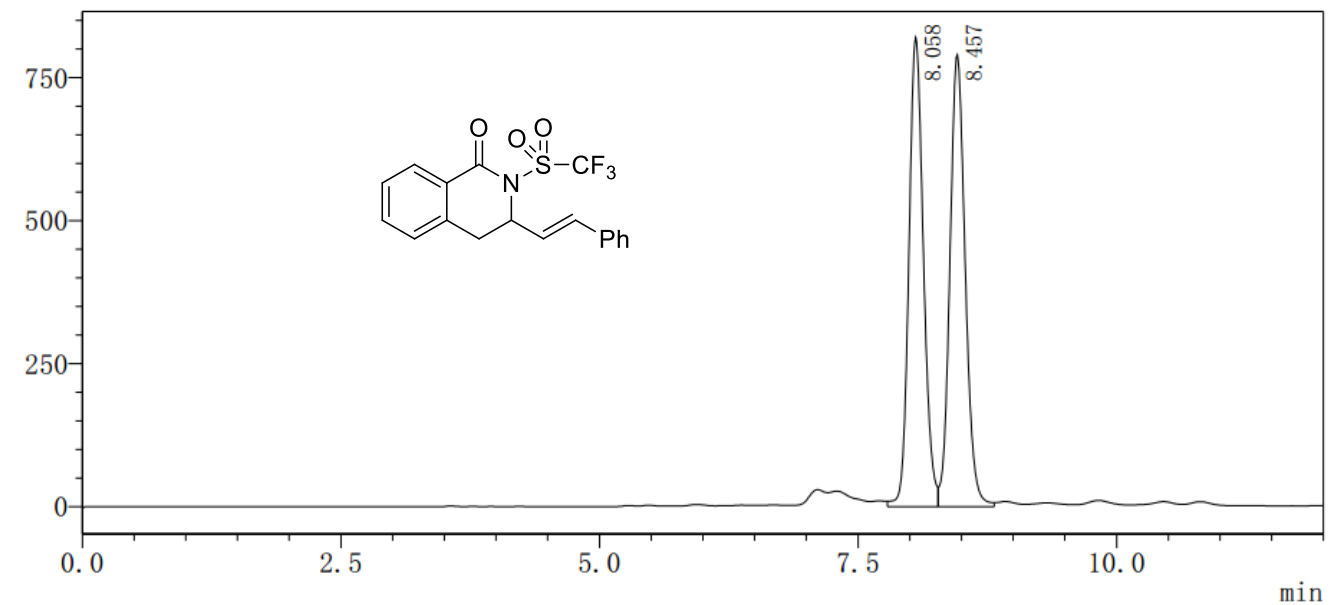

\begin{tabular}{|c|l|l|l|l|}
\hline $\begin{array}{c}\text { Peak } \\
\#\end{array}$ & $\begin{array}{c}\text { RetTime } \\
(\mathrm{min})\end{array}$ & $\begin{array}{c}\text { Area } \\
(\mathrm{mV} * \mathrm{~S})\end{array}$ & $\begin{array}{c}\text { Height } \\
(\mathrm{mV})\end{array}$ & $\begin{array}{c}\text { Area } \\
(\%)\end{array}$ \\
\hline 1 & 8.058 & 8063956 & 820037 & 49.828 \\
\hline 2 & 8.457 & 8119784 & 790096 & 50.172 \\
\hline
\end{tabular}

$\mathrm{mV}$

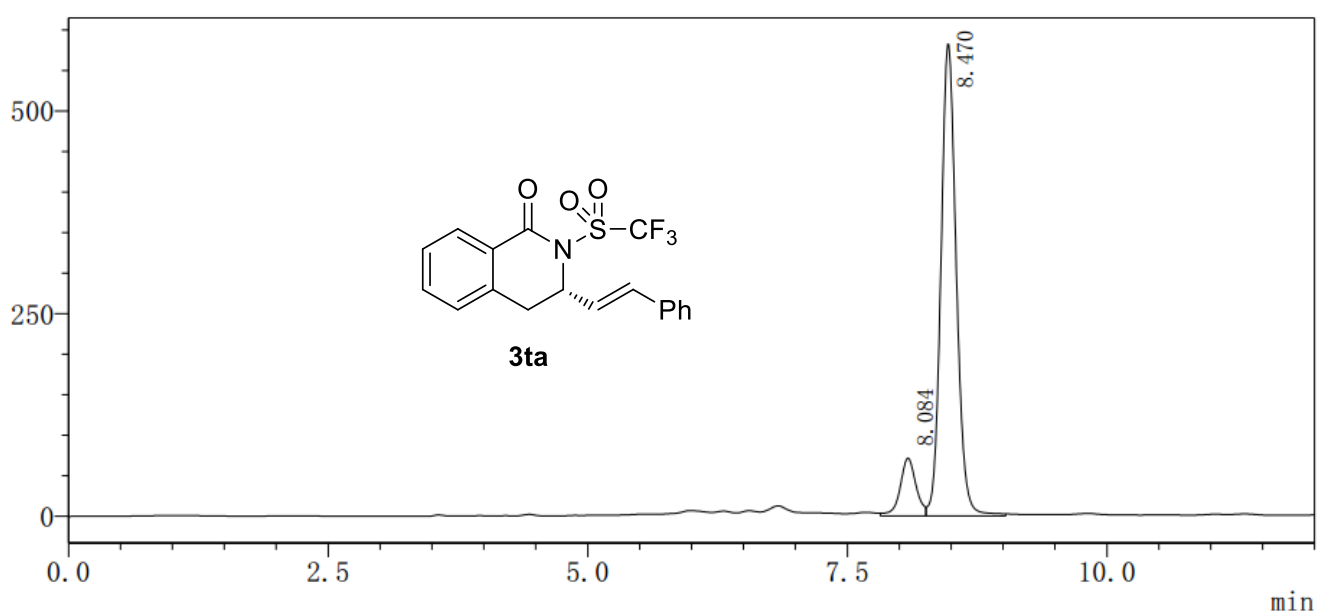

\begin{tabular}{|c|l|l|l|c|}
\hline $\begin{array}{c}\text { Peak } \\
\#\end{array}$ & $\begin{array}{c}\text { RetTime } \\
(\mathrm{min})\end{array}$ & $\begin{array}{c}\text { Area } \\
(\mathrm{mV} * \mathrm{~S})\end{array}$ & $\begin{array}{c}\text { Height } \\
(\mathrm{mV})\end{array}$ & $\begin{array}{c}\text { Area } \\
(\%)\end{array}$ \\
\hline 1 & 8.084 & 762824 & 71281 & 11.319 \\
\hline 2 & 8.470 & 5976607 & 581986 & 88.681 \\
\hline
\end{tabular}


3 ua

$\mathrm{mV}$

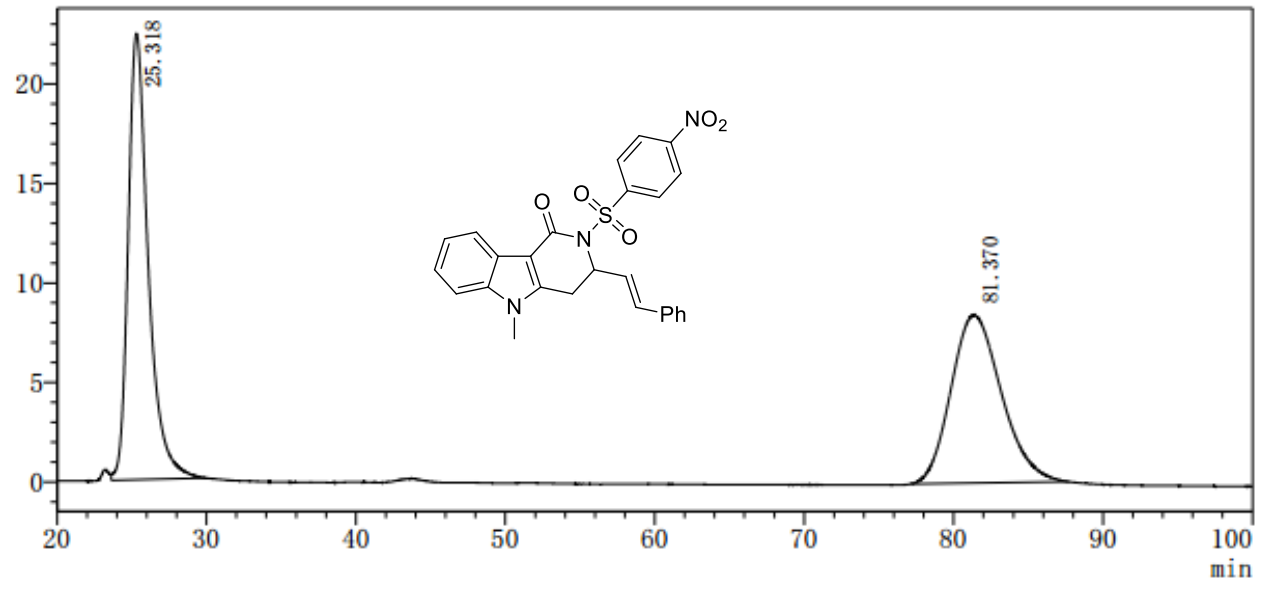

\begin{tabular}{|c|l|l|l|l|}
\hline $\begin{array}{c}\text { Peak } \\
\#\end{array}$ & $\begin{array}{c}\text { RetTime } \\
(\mathrm{min})\end{array}$ & $\begin{array}{c}\text { Area } \\
(\mathrm{mV} * \mathrm{~S})\end{array}$ & $\begin{array}{c}\text { Height } \\
(\mathrm{mV})\end{array}$ & $\begin{array}{c}\text { Area } \\
(\%)\end{array}$ \\
\hline 1 & 25.318 & 2099099 & 22421 & 51.507 \\
\hline 2 & 81.370 & 1976258 & 8432 & 48.493 \\
\hline
\end{tabular}

$\mathrm{mV}$

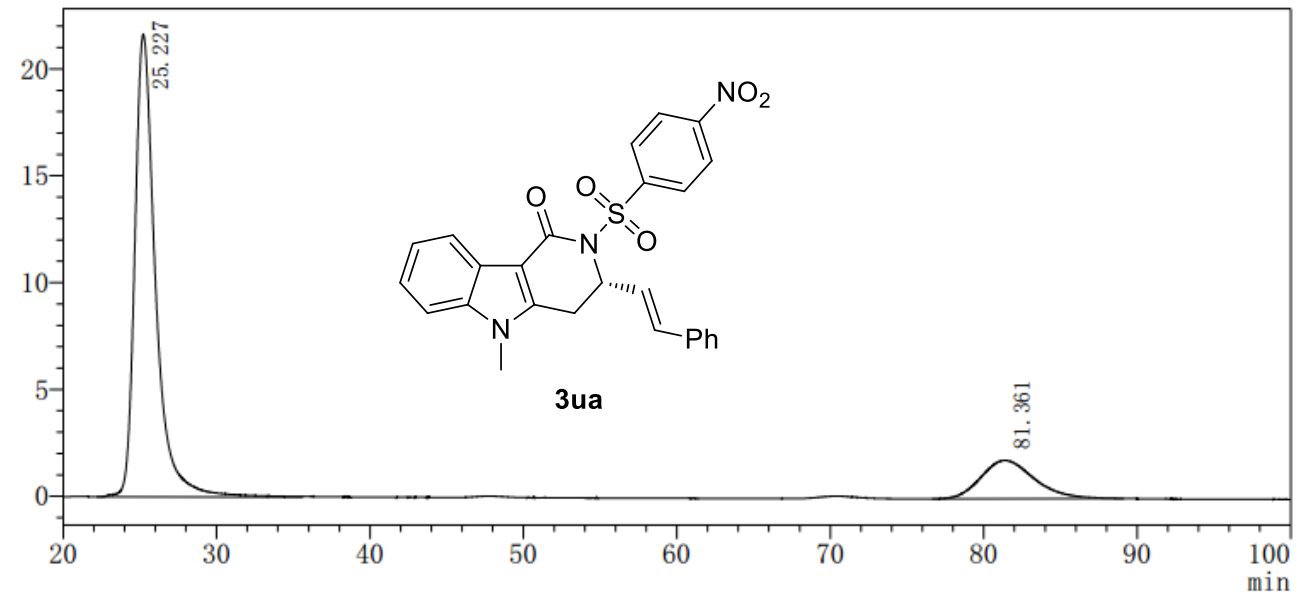

\begin{tabular}{|c|c|c|c|c|}
\hline $\begin{array}{c}\text { Peak } \\
\#\end{array}$ & $\begin{array}{c}\text { RetTime } \\
(\mathrm{min})\end{array}$ & $\begin{array}{c}\text { Area } \\
(\mathrm{mV} * \mathrm{~S})\end{array}$ & $\begin{array}{c}\text { Height } \\
(\mathrm{mV})\end{array}$ & $\begin{array}{c}\text { Area } \\
(\%)\end{array}$ \\
\hline 1 & 25.227 & 2036411 & 21628 & 82.381 \\
\hline 2 & 81.361 & 435541 & 1795 & 17.619 \\
\hline
\end{tabular}




\section{4}

$\mathrm{mV}$

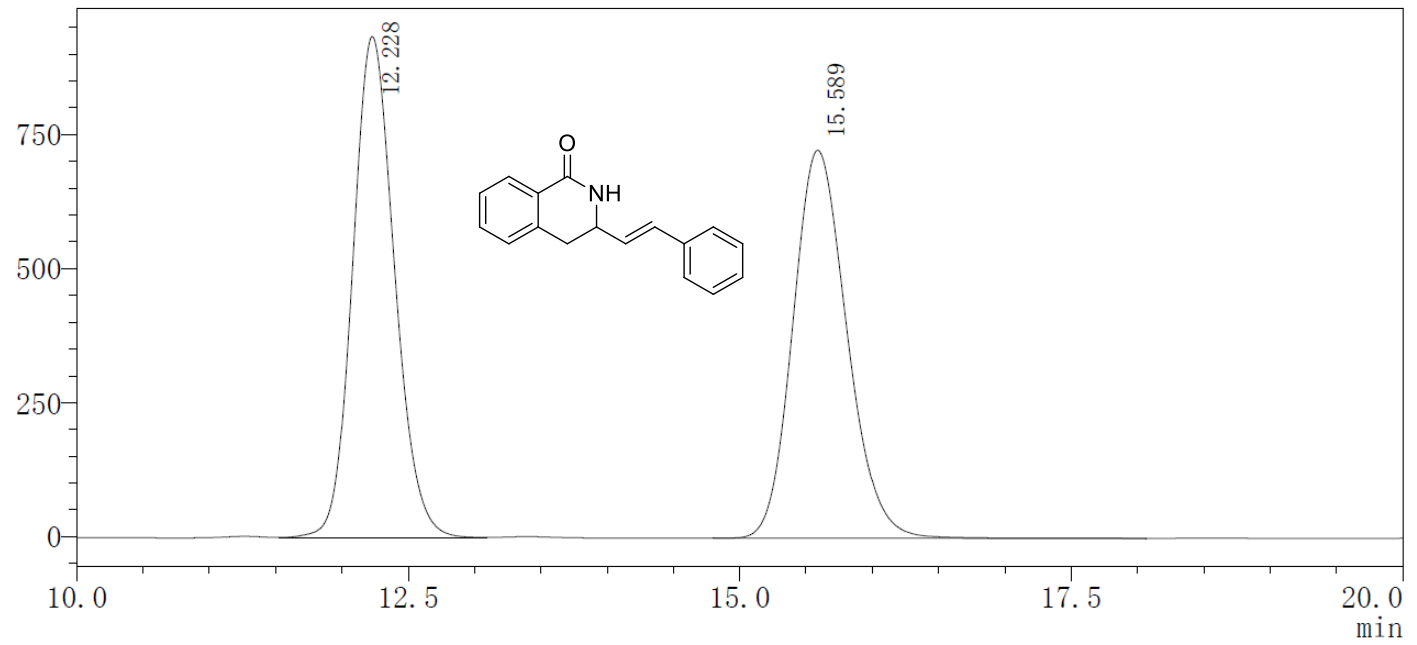

\begin{tabular}{|c|c|c|c|c|}
\hline $\begin{array}{c}\text { Peak } \\
\#\end{array}$ & $\begin{array}{c}\text { RetTime } \\
(\mathrm{min})\end{array}$ & $\begin{array}{c}\text { Area } \\
(\mathrm{mV} * \mathrm{~S})\end{array}$ & $\begin{array}{c}\text { Height } \\
(\mathrm{mV})\end{array}$ & $\begin{array}{c}\text { Area } \\
(\%)\end{array}$ \\
\hline 1 & 12.228 & 20606006 & 935645 & 50.089 \\
\hline 2 & 15.589 & 20532483 & 723929 & 49.911 \\
\hline
\end{tabular}

$\mathrm{mV}$

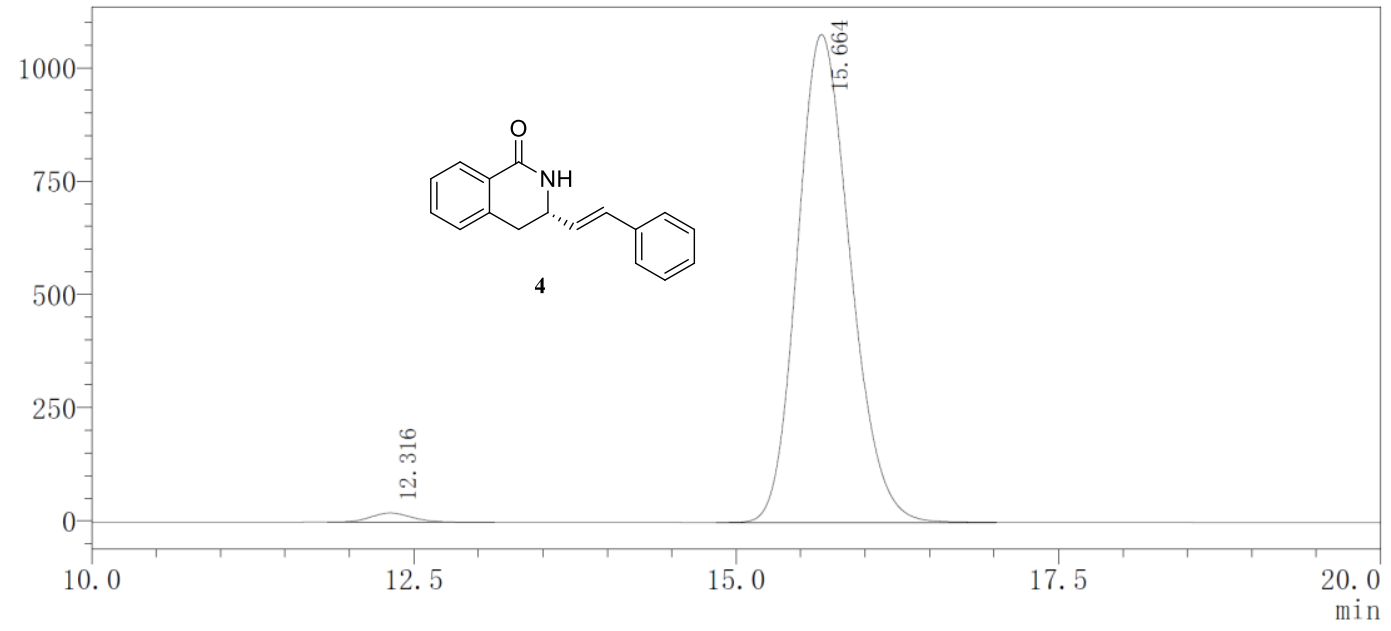

\begin{tabular}{|c|l|l|l|l|}
\hline $\begin{array}{c}\text { Peak } \\
\#\end{array}$ & $\begin{array}{c}\text { RetTime } \\
(\mathrm{min})\end{array}$ & $\begin{array}{c}\text { Area } \\
(\mathrm{mV} * \mathrm{~S})\end{array}$ & $\begin{array}{c}\text { Height } \\
(\mathrm{mV})\end{array}$ & $\begin{array}{c}\text { Area } \\
(\%)\end{array}$ \\
\hline 1 & 12.316 & 470167 & 20459 & 1.502 \\
\hline 2 & 15.664 & 30838517 & 1077268 & 98.498 \\
\hline
\end{tabular}


5

$\mathrm{mV}$

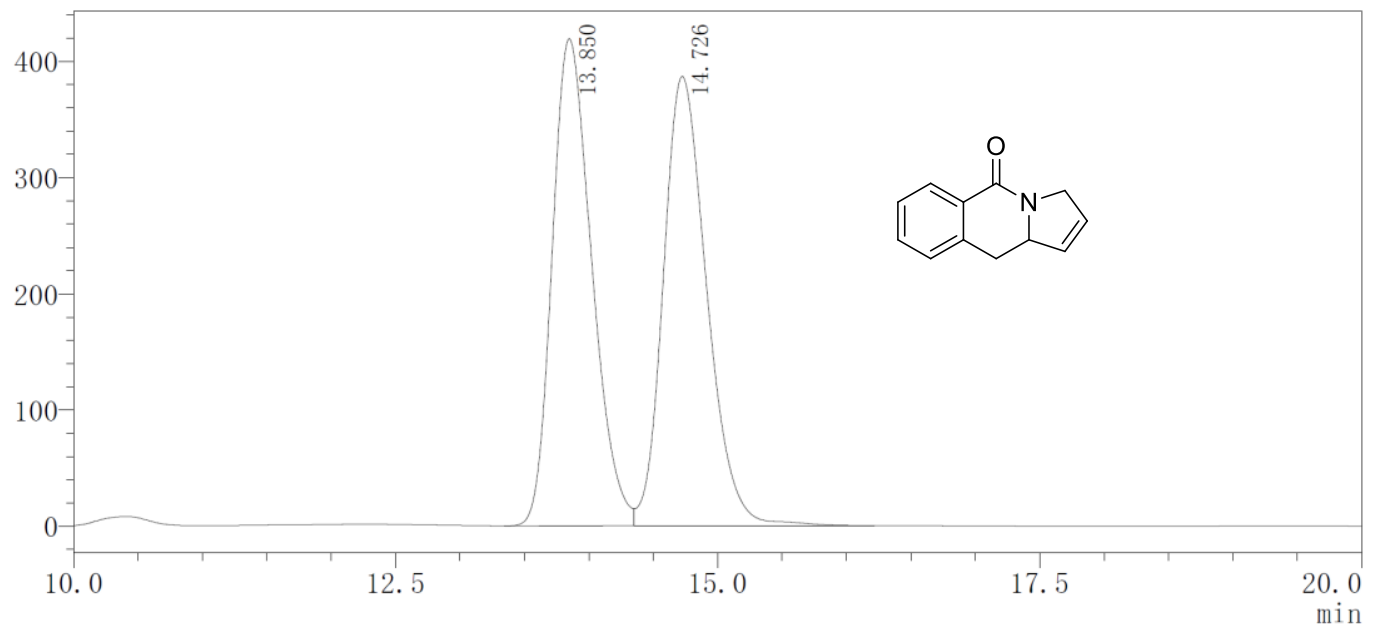

\begin{tabular}{|c|c|c|c|c|}
\hline $\begin{array}{c}\text { Peak } \\
\#\end{array}$ & $\begin{array}{c}\text { RetTime } \\
(\mathrm{min})\end{array}$ & $\begin{array}{c}\text { Area } \\
(\mathrm{mV} * \mathrm{~S})\end{array}$ & $\begin{array}{c}\text { Height } \\
(\mathrm{mV})\end{array}$ & $\begin{array}{c}\text { Area } \\
(\%)\end{array}$ \\
\hline 1 & 13.850 & 8921985 & 419182 & 49.599 \\
\hline 2 & 14.726 & 9066357 & 386718 & 50.401 \\
\hline
\end{tabular}

$\mathrm{mV}$

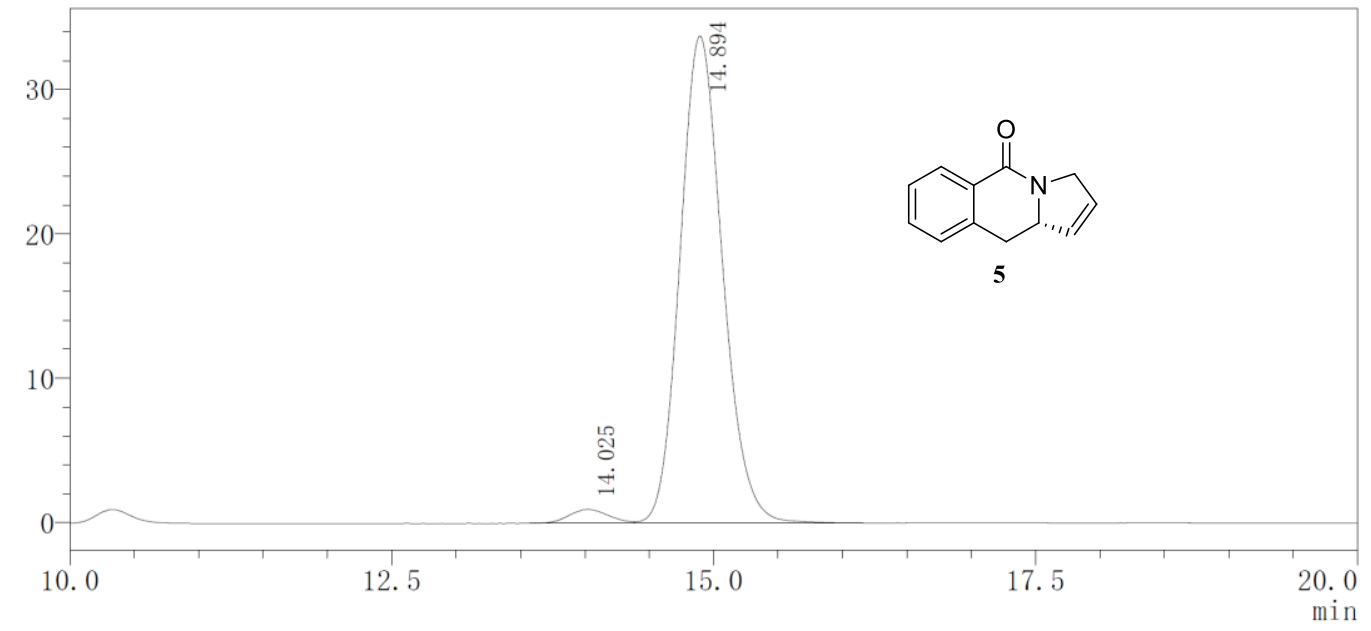

\begin{tabular}{|c|l|l|l|l|}
\hline $\begin{array}{c}\text { Peak } \\
\#\end{array}$ & $\begin{array}{c}\text { RetTime } \\
(\mathrm{min})\end{array}$ & $\begin{array}{c}\text { Area } \\
(\mathrm{mV} * \mathrm{~S})\end{array}$ & $\begin{array}{c}\text { Height } \\
(\mathrm{mV})\end{array}$ & $\begin{array}{c}\text { Area } \\
(\%)\end{array}$ \\
\hline 1 & 14.025 & 20506 & 963 & 2.574 \\
\hline 2 & 14.894 & 776224 & 33757 & 97.426 \\
\hline
\end{tabular}


6

$\mathrm{mV}$

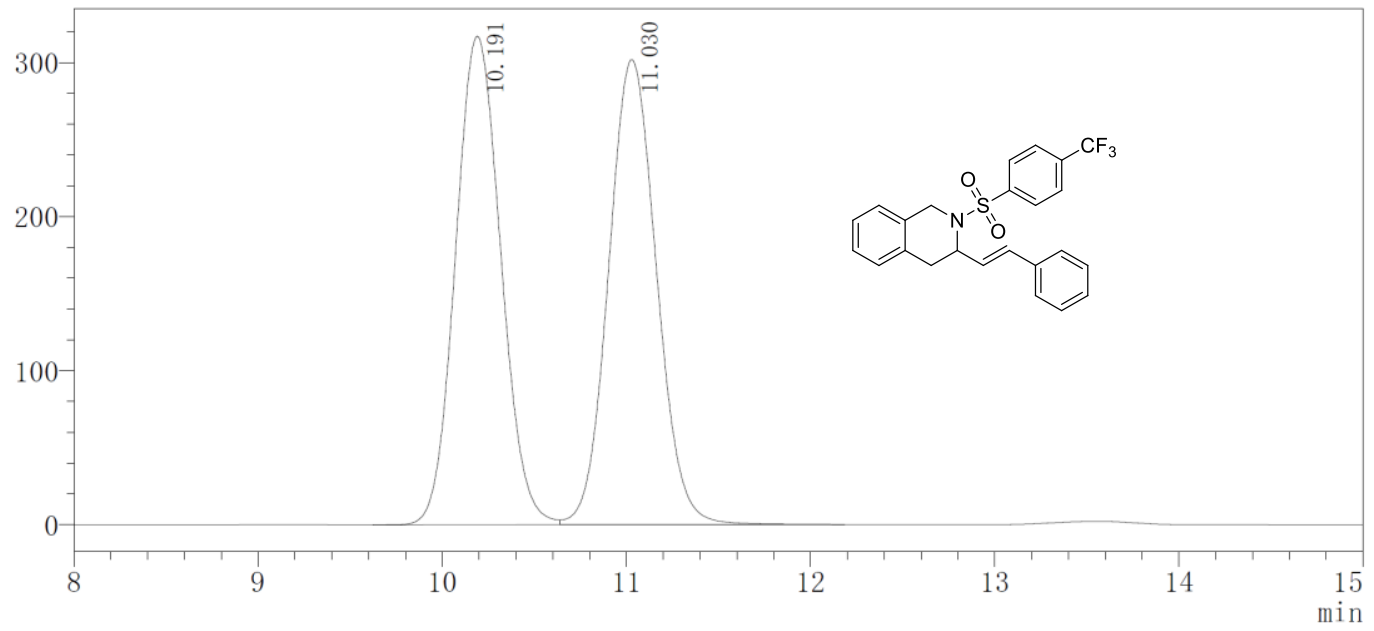

\begin{tabular}{|c|c|c|c|c|}
\hline $\begin{array}{c}\text { Peak } \\
\#\end{array}$ & $\begin{array}{c}\text { RetTime } \\
(\mathrm{min})\end{array}$ & $\begin{array}{c}\text { Area } \\
(\mathrm{mV} * \mathrm{~S})\end{array}$ & $\begin{array}{c}\text { Height } \\
(\mathrm{mV})\end{array}$ & $\begin{array}{c}\text { Area } \\
(\%)\end{array}$ \\
\hline 1 & 10.191 & 5434326 & 317248 & 49.823 \\
\hline 2 & 11.030 & 5472829 & 301983 & 50.177 \\
\hline
\end{tabular}

$\mathrm{mV}$

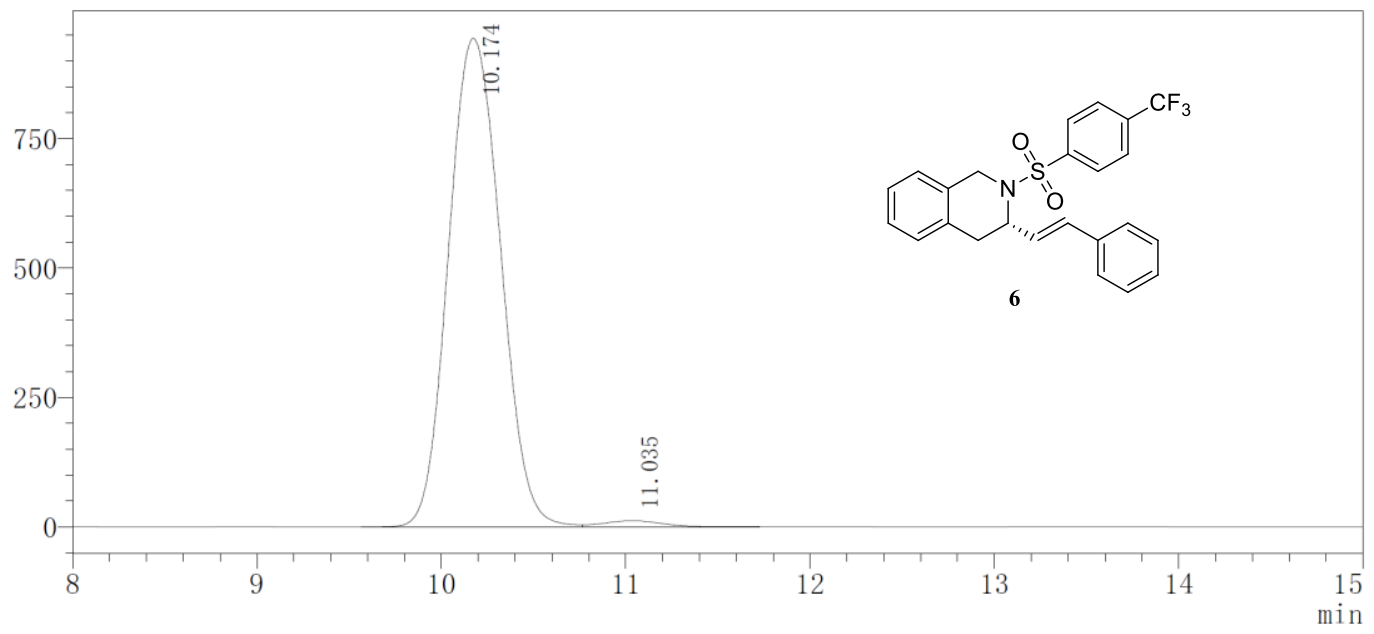

\begin{tabular}{|c|l|l|l|l|}
\hline $\begin{array}{c}\text { Peak } \\
\#\end{array}$ & $\begin{array}{c}\text { RetTime } \\
(\mathrm{min})\end{array}$ & $\begin{array}{c}\text { Area } \\
(\mathrm{mV} * \mathrm{~S})\end{array}$ & $\begin{array}{c}\text { Height } \\
(\mathrm{mV})\end{array}$ & $\begin{array}{c}\text { Area } \\
(\%)\end{array}$ \\
\hline 1 & 10.174 & 18521490 & 943638 & 98.611 \\
\hline 2 & 11.035 & 260891 & 11803 & 1.389 \\
\hline
\end{tabular}


7

$\mathrm{mV}$

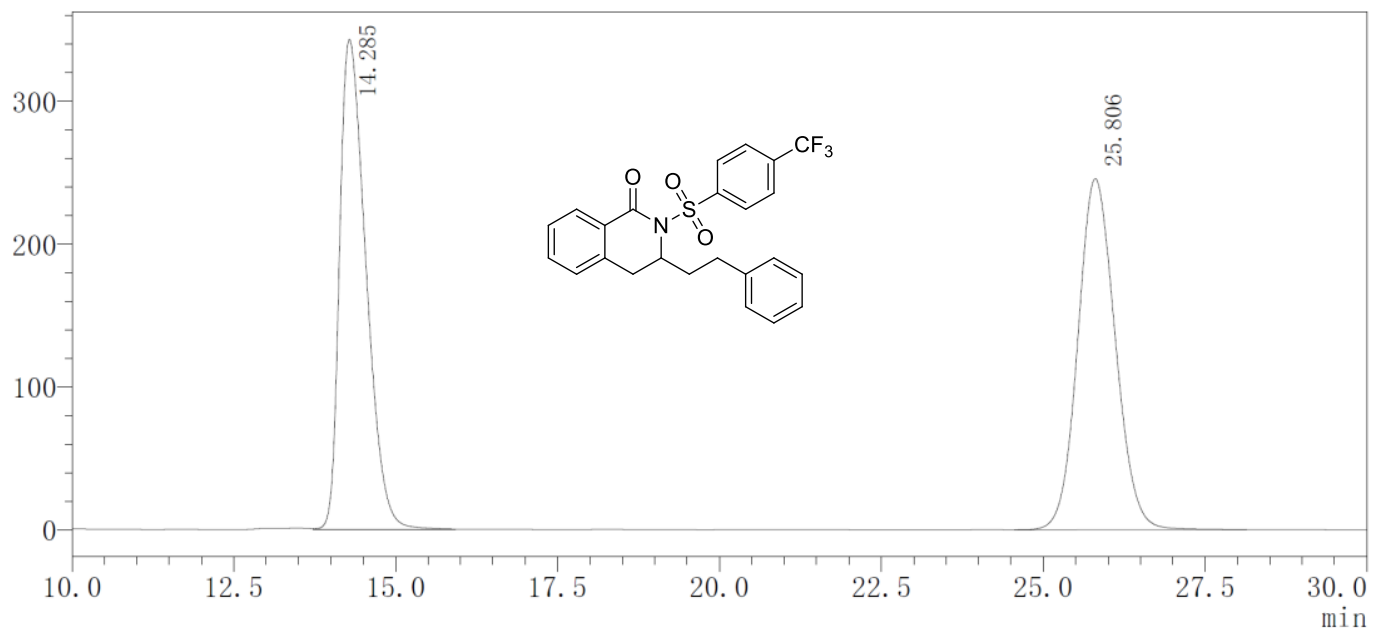

\begin{tabular}{|c|c|c|c|c|}
\hline $\begin{array}{c}\text { Peak } \\
\#\end{array}$ & $\begin{array}{c}\text { RetTime } \\
(\mathrm{min})\end{array}$ & $\begin{array}{c}\text { Area } \\
(\mathrm{mV} * \mathrm{~S})\end{array}$ & $\begin{array}{c}\text { Height } \\
(\mathrm{mV})\end{array}$ & $\begin{array}{c}\text { Area } \\
(\%)\end{array}$ \\
\hline 1 & 14.285 & 9738985 & 342996 & 49.712 \\
\hline 2 & 25.806 & 9851665 & 245673 & 50.288 \\
\hline $\mathrm{mV}$
\end{tabular}

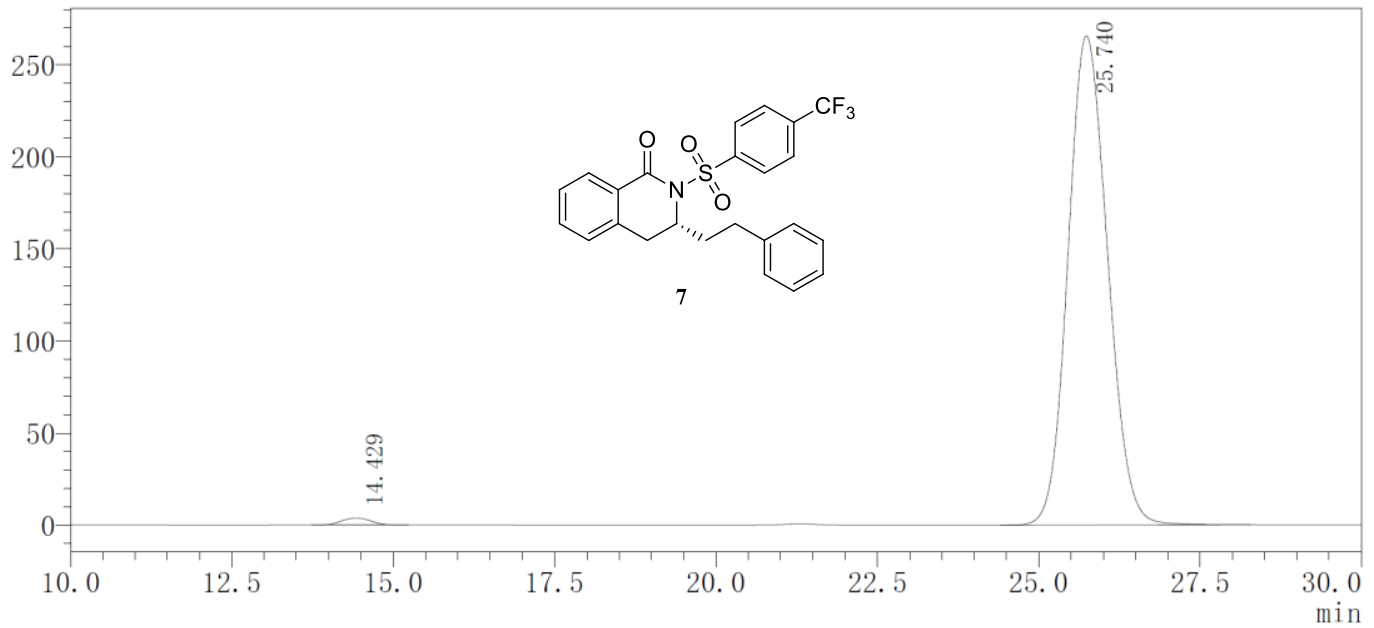

\begin{tabular}{|c|c|c|c|c|}
\hline $\begin{array}{c}\text { Peak } \\
\#\end{array}$ & $\begin{array}{c}\text { RetTime } \\
(\mathrm{min})\end{array}$ & $\begin{array}{c}\text { Area } \\
(\mathrm{mV} * \mathrm{~S})\end{array}$ & $\begin{array}{c}\text { Height } \\
(\mathrm{mV})\end{array}$ & $\begin{array}{c}\text { Area } \\
(\%)\end{array}$ \\
\hline 1 & 14.429 & 121767 & 3775 & 1.064 \\
\hline 2 & 25.740 & 11327589 & 265644 & 98.936 \\
\hline
\end{tabular}




\section{X-Ray crystallographic data}

X-ray structure of product $\mathbf{3 c a}$ (thermal ellipsoids are shown at 50\% probability level). The crystal of product 3ca was obtained by slow evaporation in $\mathrm{MeCN}$ and THF. Crystal data have been deposited to CCDC, number 1909117.
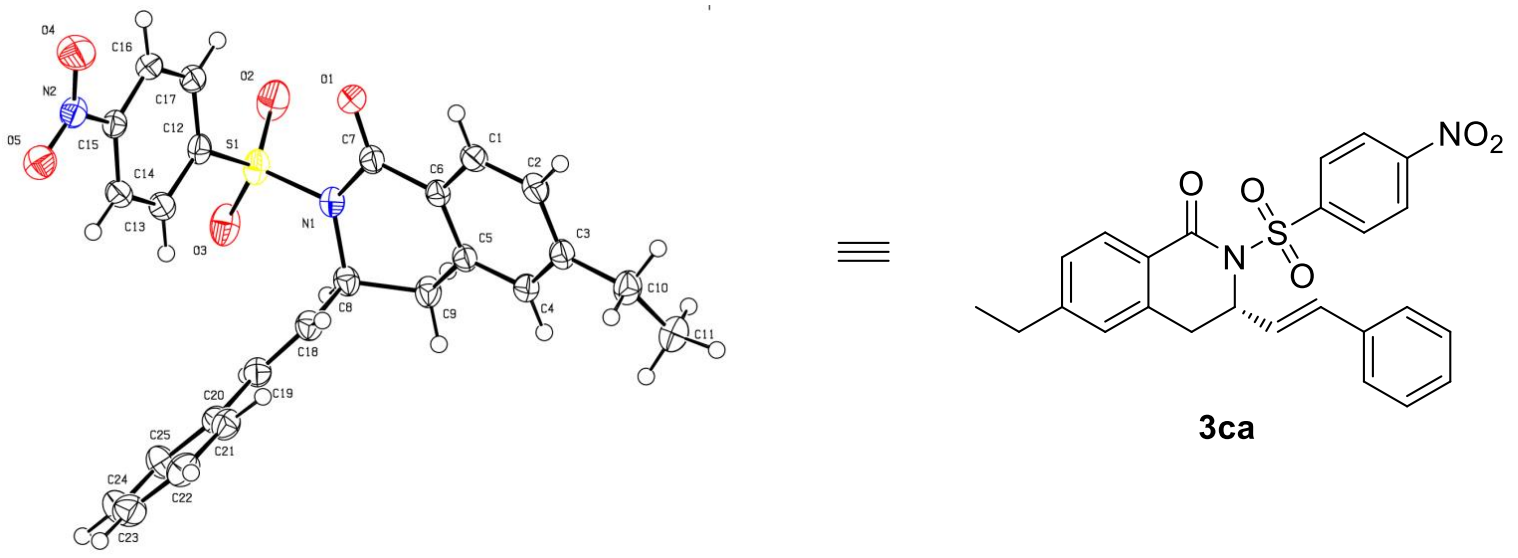

Bond precision: $\quad \mathrm{C}-\mathrm{C}=0.0022 \mathrm{~A}$

Wavelength $=0.71073$

Cell:
$\mathrm{a}=9.4331(2)$
$\mathrm{b}=12.4697(4)$
$\alpha=90^{\circ}$
$\beta=90^{\circ}$

$\mathrm{c}=18.5213(5)$

$\gamma=90^{\circ}$

Temperature: $170 \mathrm{~K}$

Calculated

2178.62(10)

P 212121

Space group

Hall group

Moiety formula

Sum formula

$\mathrm{Mr}$

Dx, $\mathrm{g} \mathrm{cm}^{-3}$

Z

$\mathrm{Mu}\left(\mathrm{mm}^{-1}\right)$

F000

F000'

h, k, 1 max

Nref
P 2ac 2ab

$\mathrm{C}_{25} \mathrm{H}_{22} \mathrm{~N}_{2} \mathrm{O}_{5} \mathrm{~S}$

$\mathrm{C}_{25} \mathrm{H}_{22} \mathrm{~N}_{2} \mathrm{O}_{5} \mathrm{~S}$

462.51

1.410

4

0.190

968.0

968.95

$13,17,26$

6661[ 3735]
Reported

2178.62(10)

P 212121

P $2 \mathrm{ac} 2 \mathrm{ab}$

$\mathrm{C}_{25} \mathrm{H}_{22} \mathrm{~N}_{2} \mathrm{O}_{5} \mathrm{~S}$

$\mathrm{C}_{25} \mathrm{H}_{22} \mathrm{~N}_{2} \mathrm{O}_{5} \mathrm{~S}$

462.50

1.410

4

0.190

968.0

$13,17,26$

6653 
Tmin, Tmax

$0.917,0.934$

$0.681,0.746$

Tmin'

0.911

Correction method $=\#$ Reported T Limits: Tmin $=0.681 \quad$ Tmax $=0.746$

AbsCorr $=$ MULTI-SCAN

Data completeness $=1.78 / 1.00$

Theta $(\max )=30.513$

$\mathrm{R}($ reflections $)=0.0313(6224)$

$\mathrm{wR} 2($ reflections $)=0.0805(6653)$

$\mathrm{S}=1.049$

Npar $=299$ 